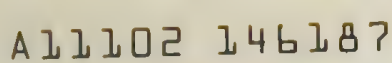

NATL INST OF STANDARDS \& TECH R.I.C.

A11102146187

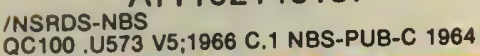

NSRDS-NBS 5

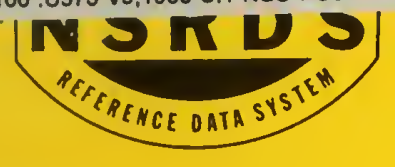

NBS

UUBLICATIONS

The Band Spectrum of

Carbon Monoxide

\section{U.S. DEPARTMENT OF COMMERCE}

NATIONAL BUREAU OF STANDARDS

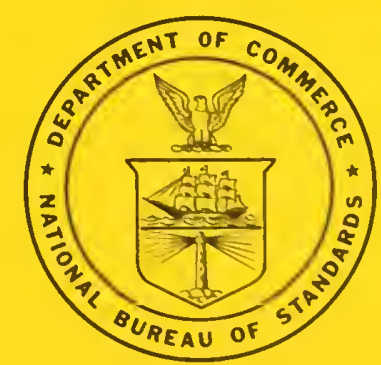

$Q C$

100

4573

No.5

1966

C 2 


\section{THE NATIONAL BUREAU OF STANDARDS}

The National Bureau of Standards is a principal focal point in the Federal Government for assuring maximum application of the physical and engineering sciences to the advancement of technology in industry and commerce. Its responsibilities include development and maintenance of the national standards of measurement, and the provisions of means for making measurements consistent with those standards; determination of physical constants and properties of materials; development of methods for testing materials, mechanisms, and structures, and making such tests as may be necessary, particularly for government agencies; cooperation in the establishment of standard practices for incorporation in codes and specifications; advisory service to government agencies on scientific and technical problems; invention and development of devices to serve special needs of the Government; assistance to industry, business, and consumers in the development and acceptance of commercial standards and simplified trade practice recommendations; administration of programs in cooperation with United States business groups and standards organizations for the development of international standards of practice; and maintenance of a clearinghouse for the collection and dissemination of scientific, technical, and engineering information. The scope of the Bureau's activities is suggested in the following listing of its three Institutes and their organizational units.

Institute for Basic Standards. Applied Mathematics. Electricity. Metrology. Mechanics. Heat. Atomic Physics. Physical Chemistry. Laboratory Astrophysics.* Radiation Physics. Radio Standards Laboratory:* Radio Standards Physics; Radio Standards Engineering. Office of Standard Reference Data.

Institute for Materials Research. Analytical Chemistry. Polymers. Metallurgy. Inorganic Materials. Reactor Radiations. Cryogenics.* Materials Evaluation Laboratory. Office of Standard Reference Materials.

Institute for Applied Technology. Building Research. Information Technology. Performance Test Development. Electronic Instrumentation. Textile and Apparel Technology Center. Technical Analysis. Office of Weights and Measures. Office of Engineering Standards. Office of Invention and Innovation. Office of Technical Resources. Clearinghouse for Federal Scientific and Technical Information.**

* Located at Boulder, Colorado, 80301.

** Located at 5285 Port Royal Road, Springfield, Virginia, 22151. 


\title{
The Band Spectrum of
}

\section{Carbon Monoxide}

\author{
Paul H. Krupenie \\ Institute for Basic Standards \\ National Bureau of Standards \\ Washington, D.G.
}

\section{NSRDS-NBS 5 \\ National Standard Reference Data Series \\ National Bureau of Standards -5 \\ (Category 3-Atomic and Molecular Properties)}

Issued July 8, 1966 
NATIONAL BUREAU OF STANDARDS

JUN 91970

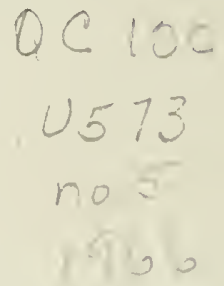

Library of Congress Catalog Card Number : 65-62765 


\section{Foreword}

The National Standard Reference Data System is a government-wide effort to give to the technical community of the United States optimum access to the quantitative data of physical science, critically evaluated and compiled for convenience. This program was established in 1963 by the President's Office of Science and Technology, acting upon the recommendation of the Federal Council for Science and Technology. The National Bureau of Standards has been assigned responsibility for administering the effort. The general objective of the System is to coordinate and integrate existing data evaluation and compilation activities into a systematic, comprehensive program, supplementing and expanding technical coverage when necessary, establishing and maintaining standards for the output of the participating groups, and providing mechanisms for the dissemination of the output as required.

The NSRDS is conducted as a decentralized operation of nation-wide scope with central coordination by NBS. It comprises a complex of data centers and other activities, carried on in government agencies, academic institutions, and nongovernmental laboratories. The independent operational status of existing critical data projects is maintained and encouraged. Data centers that are components of the NSRDS produce compilations of critically evaluated data, critical reviews of the state of quantitative knowledge in specialized areas, and computations of useful functions derived from standard reference data.

For opera tional purposes, NSRDS compilation activities are organized in to seven categories as listed below. The data publications of the NSRDS, which may consist of monographs, looseleaf sheets, computer tapes, or any other useful product, will be classified as belonging to one or another of these categories. An additional "General" category of NSRDS publications will include reports on detailed classification schemes, lists of compilations considered to be Standard Reference Data, status reports, and similar material. Thus, NSRDS publications will appear in the following eight categories:

Category
1
2
3
4
5
6
7
8

\section{Title}

General
Nuclear Properties
Atomic and Molecular Properties
Solid State Properties
Thermodynamic and Transport Properties
Chemical Kinetics
Colloid and Surface Properties
Mechanical Properties of Materials

The present compilation is in category 3 of the above list. It constitutes the fifth publication in a new NBS series known as the National Standard Reference Data Series.

A. V. Astrn, Director. 


\section{Contents}

1. Introduction -.......................

2. Electronic structure of $\mathrm{CO}$ and $\mathrm{CO}^{+}$

2.1 Electronic structure

2.2 Numerical calculations................

a. Semiempirical

b. Single configuration

c. Configuration interaction. .........

2.3 Rydberg states and Rydberg series .....-

3. Electronic spectrum of $\mathrm{CO}$ and $\mathrm{CO}^{+}$

$3.1 \mathrm{~A}^{1} \Pi-\mathrm{X}^{1} \Sigma^{+}$Fourth Positive system (2800$1140 \AA$ A) $\mathrm{R}$

$3.2 \mathrm{~B}^{1} \Sigma^{+}-\mathrm{A}^{1} \Pi$ Angstrom system $(6620-4120$ A) $\mathrm{V}$

$3.3 \mathrm{C}^{1} \Sigma^{+}-\mathrm{A}^{1} \Pi$ Herzberg system (5710-3680 A) $\mathrm{V}$

3.4 Hopfield-Birge systems $(1810-950 \AA)$. . - a. $\mathrm{B}^{1} \Sigma^{+}, \mathrm{C}^{1} \Sigma^{+}, \mathrm{E}^{1} \Pi, \mathrm{F}\left({ }^{1} \Sigma^{+}\right), \mathrm{G}\left({ }^{1} \Pi\right)$, and $b^{3} \Sigma^{+}-\mathrm{X}^{1} \Sigma^{+}+\ldots$ b. $a^{\prime} \Sigma^{+}-\mathrm{X}^{1} \Sigma^{+}(1810-1280 \AA) \mathrm{R}$

$3.5 a^{3} \Pi-\mathrm{X}^{1} \Sigma^{+}$Cameron system $(5800-1770$ $\AA$ ) $R$

3.6 $b^{3} \Sigma^{+}-a^{3} \Pi$ Third Positive system (3820$2660 \AA) \mathrm{V}$

$3.7 c^{3} \Sigma^{+}-a^{3} \Pi 3 \mathrm{~A}$ system $(2710-2300 \AA) \mathrm{V}$ -

$3.8 a^{\prime} \Sigma^{+}-a^{3} \Pi$ Asundi system (8590-3900 $\AA$ )

R _.

$3.9 d^{3} \Delta_{i}-a^{3}$ II Triplet system $(7500-3770 \AA)$

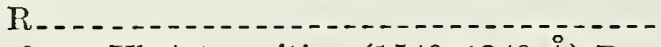

$3.10 e^{3} \Sigma^{-}-\mathrm{X}^{1} \Sigma^{+}$transition $(1540-1240 \AA) \mathrm{R}_{\text {- }}$

$3.11 \mathrm{C}^{1} \Sigma^{+}-a^{\prime 3} \Sigma^{+}$Knauss system (3250-2930 A) $\mathrm{V}$.

3.12 Kaplan bands $(2750-2520 \AA)$

$3.13 d^{3} \Delta_{i}-\mathrm{X}^{1} \Sigma^{+}$transition $(1620-1230 \AA) \mathrm{R}_{--}$

$3.14 e^{3} \Sigma^{-}-a^{3} \Pi$ Herman system $(5430-4270 \AA)$ R _...............................

$3.15 \mathrm{E}_{0} \Sigma^{+} \Sigma^{+}-\mathrm{X}^{1} \Sigma^{+},{ }^{1} \Pi-\mathrm{X}^{1} \Sigma^{+}$, and several unidentified transitions in the vacuum UV region $(1180-930 \AA)$. . . . . . $3.16 f^{3} \Sigma^{+}-a^{3} \Pi$ transition $(2980-2670 \AA) R_{\ldots} .$. $3.17 \mathrm{I}^{1} \Sigma^{-}-\mathrm{X}^{1} \Sigma^{+}$transition $(1520-1460 \AA) \mathrm{R}_{- \text {- }}$

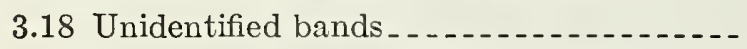

$3.19 \mathrm{P}, \mathrm{Q}, \mathrm{R}, \mathrm{S}, \mathrm{T}-\mathrm{X}^{1} \Sigma^{+}$Tanaka systems (800-630 $\AA)$ R . . . .

3.20 Rydberg series $(940-630 \AA)$.

$3.21 \mathrm{~B}^{2} \Sigma^{+}-\mathrm{X}^{2} \Sigma^{+}$First Negative system of $\mathrm{CO}^{+}(3150-1800 \AA) \cdot \mathrm{R}$

$3.22 \mathrm{~A}^{2} \Pi_{i}-\mathrm{X}^{2} \Sigma^{+}$Comet Tail system of $\mathrm{CO}^{+}$

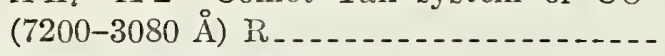

$3.23 \mathrm{~B}^{2} \Sigma^{+}-\mathrm{A}^{2} \Pi_{i}$ Baldet-Johnson intercombination system of $\mathrm{CO}^{+}(4240-3310 \AA) \mathrm{V}$.

4. Perturbations

$4.1 \mathrm{~A}^{1} \Pi$ state

a. $v=0$

b. $v=1$

c. $v=2$

d. $v=3$

e. $v=4$

f. $v=5$

g. $v=6$
Page

1

2

4

4

4

4

5

5

6

8

9

10

10

10

11

12

13

15

16

17

17

17

17

17

18

18

19

19

19

20

21

22

22

22

22

23

24

24

24

24

24 h. $v=7$

i. $v=8$

j. $v=9$

k. $v=10$

l. $v=11$

m. $v=12$

n. $v=13$

4.2 E1II state

$4.3 a^{3}$ II state

$4.4 d^{3} \Delta_{i}$ state

$4.5 f^{3} \Sigma^{+}$state

$4.6 b^{3} \Sigma^{+}$state

$4.7 \mathrm{~A}^{2} \Pi_{i}$ state of $\mathrm{CO}^{+}$

a. $v=5$

b. $v=10$

5. Dissociation energies, predissociations, and convergence limits. . . . . - . . .

5.1 Dissociation energy of CO..........-

5.2 Predissociations and convergence limits.-

a. Predissociation of the $\mathrm{B}^{1} \Sigma^{+}$state..... -

b. Predissociation of the $b^{3} \Sigma^{+}$state......

c. Predissociation of the $\mathrm{C}^{1} \Sigma^{+}$state.....-

d. Suspected predissocation of the $c^{3} \Sigma^{+}$ state............................

e. Convergence limit of the $a^{\prime 3} \Sigma^{+}$state..-

f. Convergence limit of the $F\left(\Sigma^{+}\right)$state.

5.3 Dissociation energy of $\mathrm{CO}^{+}$, ionization potentials and Rydberg series..........

5.4 Dissociation energy of $\mathrm{CO}^{2+} \ldots \ldots \ldots . .$.

6. The vibration-rotation spectrum of $\mathrm{CO}$

$6.1 \Delta v=1$ sequence......................

6.2 Overtone sequence and other overtone

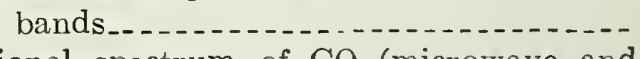

7. Rotational spectrum of $\mathrm{CO}$ (microwave and far infrared) ............................

7.1 Rotational transitions in $\mathrm{CO}$

7.2 Stark effect..........................

7.3 Zeeman effect.....................

7.4 Quadrupole hỵperfine structure .........

8. Raman, Stark, and Zeeman effects in electronic spectra of $\mathrm{CO}$ and $\mathrm{CO}^{+} \ldots$

8.1 Raman effect.......................

8.2 Stark effect.............

8.3 Zeeman effect.

9. Molecular energy levels and potential energy curves.................................

10. Transition probabilities and lifetimes (electronic spectra) _............................

$10.1 \mathrm{~A}^{1} \Pi-\mathrm{X}^{1} \Sigma^{+}$Fourth Positive system....-

$10.2 \mathrm{~B}^{1} \Sigma^{+}-\mathrm{A}^{1} \Pi$ Ångstrom system

$10.3 \mathrm{C}^{1} \Sigma^{+}-\mathrm{A}^{1}$ II Herzberg system-_.......

$10.4 b^{3} \Sigma^{+}-a^{3} \Pi$ Third Positive system . . . . - -

$10.5 a^{3} \Sigma^{+}-a^{3} \Pi$ Asundi system

$10.6 a^{3} \Pi-\mathrm{X}^{1} \Sigma^{+}$Cameron system $\ldots . . . . . . .$.

$10.7 b^{3} \Sigma^{+}-\mathrm{X}^{1} \Sigma^{+}$Hopfield-Birge system -..-. -

$10.8 a^{\prime 3} \Sigma^{+}-\mathrm{X}^{1} \Sigma+$ Hopfield-Birge system....

$\left.10.9 d^{3}\right\lrcorner_{i}-a^{3}$ II Triplet system . . . . . . . . ...

$10.10 \mathrm{~A}^{2} \Pi_{i}-\mathrm{X}^{2} \Sigma^{+}\left(\mathrm{CO}^{+}\right)$Comet Tail system -

$10.11 \mathrm{~B}^{2} \Sigma^{+}-\mathrm{X}^{2} \Sigma^{+}\left(\mathrm{CO}^{+}\right)$First Negative system.
Page

24

25

25

25

25

25

25

25

26

26

26

26

26

27

27

27

28

28

28

29

29

29

30

30

31

31

31

31

32

32

32

32

33

33

33

33

33

34

35

35

35

35

35

36

36

36

36

36

36

37 


\section{Contents-Continued}

$10.12 \mathrm{~B}^{2} \Sigma^{+}-\mathrm{A}^{2} \Pi_{i}\left(\mathrm{CO}^{+}\right)$Baldet-Johnson system

10.13 Ionization of $\mathrm{CO}$

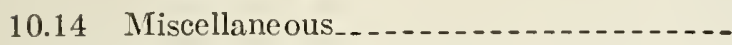

11. Summary and conclusion.............. 37
37

37

37

12. Tables and figures.

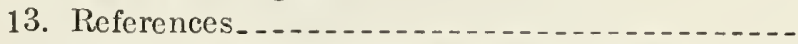
Appendix A-notation and terminology _...... Appendix B-physical constants and conversion factors.

Page

$38 \mathrm{a}$

78

85

87
1. Molecular constants, electron configurations, and dissociation products for the electronic states of $\mathrm{CO}$ and $\mathrm{CO}^{+}$. Supplement: Rydberg states .

2. Electron configurations for states of $\mathrm{CO}$ and $\mathrm{CO}^{+}$

3. Band heads of the $A^{1} \Pi-X^{1} \Sigma^{+}$Fourth Positive system (R) -

(a) Emission

(b) Absorption

4. Deslandres table for the band origins of the $\mathrm{A}^{1} \mathrm{I}-\mathrm{X}^{1} \Sigma^{+}$Fourth Positive system ..........

5. Band heads and origins of the $A^{1} \Pi-X^{1} \Sigma^{+}$Fourth Positive system of $\mathrm{C}^{13} \mathrm{O}^{16}(\mathrm{R})$

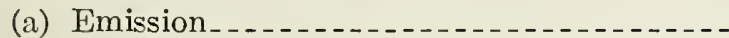

(b) Absorption

6. Band heads and origins of the $\mathrm{B}^{1} \mathrm{\Sigma}^{+} \rightarrow \mathrm{A}^{1} \mathrm{II}$ Angstrom system (V)

7. Band heads and origins of the $\mathrm{B}^{1} \Sigma^{+} \rightarrow \mathrm{A}^{1} \Pi$ system of $\mathrm{C}^{13} \mathrm{O}^{16}(\mathrm{~V})$

8. Band heads and origins of the $\mathrm{C}^{1} \Sigma^{+} \rightarrow \mathrm{A}^{1} \Pi$ Herzberg system (V)

9. Band heads of the Hopfield-Birge systems

(a) $\mathrm{B}^{1} \Sigma^{+} \leftrightarrow \mathrm{X}^{1} \Sigma^{+} \ldots \ldots$

(b) $\mathrm{C}^{1} \Sigma^{+} \leftrightarrow \mathrm{X}^{1} \Sigma^{+} \ldots \ldots \ldots$

(c) $\mathrm{E}^{1} \mathrm{II} \leftrightarrow \mathrm{X}^{1} \Sigma^{+} \ldots \ldots \ldots$

(d) $\mathrm{F}\left({ }^{1} \Sigma^{+}\right) \leftarrow \mathrm{X}^{1} \Sigma^{+} \ldots \ldots \ldots$

(e) $\mathrm{G}\left({ }^{1} \Pi\right) \leftarrow \mathrm{X}^{1} \Sigma^{+} \ldots \ldots \ldots \ldots$

(f) $b^{3} \Sigma^{+} \leftrightarrow \mathrm{X}^{1} \Sigma^{+}-$

10. Band heads and origins of the $a^{\prime 3} \Sigma^{+} \leftarrow \mathrm{X}^{1} \Sigma^{+}$ Hopfield-Birge system (R) _................

11. Band heads of the $a^{3} \Pi-\mathrm{X}^{1} \Sigma+$ Cameron system (R)

(a) Emission.............

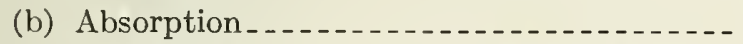

12. Band origins of the $a^{3} \Pi-\mathrm{X}^{1} \Sigma^{+}$Cameron system.

13. Band heads of the $b^{3} \Sigma^{+} \rightarrow a^{3} \Pi$ Third Positive system (V)

14. Band heads of the $c^{3} \Sigma^{+} \rightarrow a^{3} \Pi 3 \mathrm{~A}$ system $(\mathrm{V})$-.

15. Band heads of the $a^{\prime} \Sigma^{+} \rightarrow a^{3} \Pi$ Asundi system

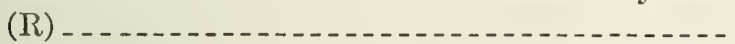

16. Band heads of the $d^{3} \Delta_{i} \rightarrow a^{3} \Pi$ Triplet system (R) _.........

(a) Irlentified bands............

(b) Unassigned bands

17. Band origins of the $d^{3} \Delta_{i}-a^{3} \Pi$ Triplet system --

18. Band heads and origins of the $e^{3 \Sigma^{-}} \leftarrow \mathrm{X}^{1} \mathrm{\Sigma}^{+}$system (R)

19. Band heads of the $\mathrm{C}^{1} \Sigma^{+} \rightarrow a^{\prime 3} \mathrm{\Sigma}^{+}$Kinauss sys

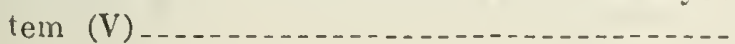

20. Band heads of the Kaplan system (R) ......

21. Band heads of the $d^{3} \Delta_{i} \leftarrow \mathrm{X}^{1} \Sigma^{+} \operatorname{system}(\mathrm{R}) \ldots$
$38 \mathrm{a}$

40

40

41

42

43

43

43

43

43

43

44

44

44

44

44

44

44

44

45

45

45

45

46

46

47

49

49

50

50

50

50
22. Band heads of the $e^{3} \Sigma^{-} \rightarrow a^{3} \Pi$ Herman system (R)

23. Band heads of the $\mathrm{E}_{0}^{1} \Sigma^{+} \rightarrow \mathrm{X}^{1} \Sigma^{+}$system $(\mathrm{R})$

24. Band heads of the ${ }^{1} \Pi \rightarrow \mathrm{X}^{1} \Sigma^{+}$system $(\mathrm{R}) \ldots$

25. Band heads of the $f^{3} \Sigma^{+} \rightarrow a^{3} \Pi$ system $(\mathrm{R})$

26. Band heads of the Tanaka systems (absorption)

(a) $\mathrm{P} \leftarrow \mathrm{X}^{1} \Sigma^{+} \ldots \ldots$

(b) $\mathrm{Q} \leftarrow \mathrm{X}^{1} \Sigma^{+} \ldots \ldots \ldots 52$

(c) $\mathrm{R} \leftarrow \mathrm{X}^{1} \Sigma^{+} \ldots \ldots \ldots 2$

(d) $\mathrm{S} \leftarrow \mathrm{X}^{1} \Sigma^{+} \ldots \ldots$

(e) $\mathrm{T} \leftarrow \mathrm{X}^{1} \Sigma^{+} \ldots \ldots \ldots \ldots 2$

27. $\mathrm{B}^{2} \Sigma^{+}\left(\mathrm{CO}^{+}\right) \leftarrow \mathrm{X}^{1} \Sigma^{+}$" $\beta$ " Rydberg series_..... 52

28. $\mathrm{A}^{2} \Pi_{i}\left(\mathrm{CO}^{+}\right) \leftarrow \mathrm{X}^{1} \Sigma^{+}$" $\alpha$ " Rydberg series . . . . . . 53

29. $\mathrm{X}^{2} \Sigma^{+}\left(\mathrm{CO}^{+}\right) \leftarrow \mathrm{X}^{1} \Sigma^{+}$Rydberg series........... 53

30. Band heads of the $\mathrm{B}^{2} \Sigma^{+} \rightarrow \mathrm{X}^{2} \Sigma^{+}$First Negative system of $\mathrm{CO}^{+}(\mathrm{R})$

31. Band origins of the $\mathrm{B}^{2} \Sigma^{+}-\mathrm{X}^{2} \Sigma^{+}$First Negative system of $\mathrm{CO}^{+}$. , . ${ }_{3}^{2}$ 3

32. Band heads of the $\mathrm{A}^{2} \Pi_{i} \rightarrow \mathrm{X}^{2} \Sigma^{+}$Comet Tail system of $\mathrm{CO}^{+}(\mathrm{R}) \ldots \ldots$

33. Band origins of the $\mathrm{A}^{2} \Pi_{2}-\mathrm{X}^{2} \Sigma^{+}$Comet Tail

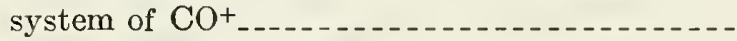

34. Band heads of the $\mathrm{B}^{2} \Sigma^{+} \rightarrow \mathrm{A}^{2} \Pi_{i}$ Baldet-Johnson system of $\mathrm{CO}^{+}(\mathrm{V})$

35. Miscellaneous unclassified bands............

(a) Band heads observed together with the $\mathrm{A}^{1} \mathrm{II}-\mathrm{X}^{1} \Sigma^{+}$Fourth Positive system (Emission) - _. - _.

(b) Band heads observed in absorption

(c) Band heads observed with the $a^{\prime 3} \Sigma^{+} \rightarrow a^{3} \Pi$

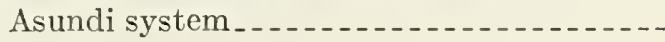

(d) Band heads observed in emission by Tschulanovsky and Gassilevitch........

(e) Triplet bands observed in emission .......

(f) Unclassified absorption maxima_........

(g) Unclassified absorption bands............

(h) Emission and absorption bands of Inand_-

(i) Band heads observed together with the $\mathrm{A}^{2} \Pi_{i} \rightarrow \mathrm{X}^{2} \mathrm{\Sigma}^{+}$Comet Tail system of $\mathrm{CO}^{+}+-$

(j) Band heads observed with the $\mathrm{B}^{2} \mathrm{~S}^{+} \rightarrow \mathrm{A}^{2} \mathrm{II}_{i}$ system of $\mathrm{CO}^{+} \ldots \ldots \ldots-\ldots$

36. Rotational constants for the $\mathrm{X}^{1} \mathrm{\Sigma}^{+}$state.....

37. Rotational constants for the ${ }^{1} I I$ state._.....

38. Rotational constants for the $\mathrm{B}^{1} \mathrm{~s}^{+}$state.....- 


\section{Tables-Continued}

39. Rotational constants for the $\mathrm{C}^{1} \Sigma^{+}$state $\ldots . . . .58$

40. Rotational constants for the $\mathrm{E}^{1} \Pi$ state_._._._. $\quad 59$

41. Rotational constants for the $a^{\prime 3} \Sigma^{+}$state_..... $\quad 59$

42. Rotational constants for the $a^{3}$ II state...... $\quad 59$

43. Rotational constants for the $b^{3} \Sigma^{+}$state _.... $\quad 59$

44. Rotational constants for the $c^{3} \Sigma^{+}$state_._._. $\quad 59$

45. Rotational constants for the $d^{3} \Delta_{i}$ state_-_-_-_ $\quad 59$

46. Rotational constants for the $e^{3} \Sigma^{-}$state_._._. $\quad 59$

47. Rotational constants for the $\mathrm{E}_{0}^{1} \Sigma^{+}$state $\ldots \ldots$

48. Rotational constants for the ${ }^{1} \Pi$ state._._._. $\quad 59$

49. Rotational constants for the $f^{3} \Sigma^{+}$state_._._._- 59

50. Rotational constants for the $\mathrm{X}^{2} \Sigma^{+}$state of $\mathrm{CO}^{+}$

51. Rotational constants for the $\mathrm{A}^{2} \Pi_{i}$ state of $\mathrm{CO}^{+} \ldots \ldots \ldots$

52. Rotational constants for the $\mathrm{B}^{2} \Sigma^{+}$state of

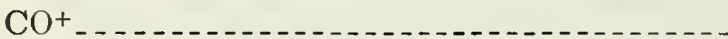

53. Doublet splitting constants for states of $\mathrm{CO}^{+}-$

54. Perturbations of the electronic states of $\mathrm{CO}$ and $\mathrm{CO}^{+}$

(a) $\mathrm{A}^{1} \mathrm{II}$ state

(b) E'II state $\ldots \ldots$

(c) $a^{3}$ II state $\ldots \ldots \ldots$

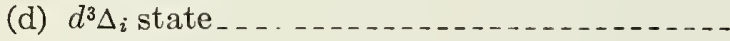

(e) $f^{3} \Sigma+$ state $\ldots \ldots \ldots \ldots$

(f) $b^{3} \Sigma^{+}$state $\ldots \ldots \ldots$

(g) $\mathrm{A}^{2} \Pi_{i}$ state of $\mathrm{CO}^{+} \ldots \ldots \ldots$

55. Observed predissociations in $\mathrm{C}^{12} \mathrm{O}^{16}$ and $\mathrm{C}^{13} \mathrm{O}^{16}$ -

56. Vibration-rotation bands of $\mathrm{CO}$ in the infrared region.

57. Dipole moments for states of $\mathrm{CO}$ and $\mathrm{CO}^{+}$

58. Rotational frequencies, rotational constants, and magnetic moments of carbon monoxide..-

59. Hyperfine structure

60. (a) Franck-Condon factors for the $\mathrm{A}^{1} \Pi-\mathrm{X}^{1} \Sigma^{+}$ Fourth Positive system

(b) $r$-centroids ( $\AA$ ) for the $A^{1} \Pi-\mathrm{X}^{1} \Sigma+$ Fourth Positive system

61. Franck-Condon factors, band strengths, $r$ - centroids, and $I_{\infty}$ for the $\mathrm{B}^{1} \Sigma^{+}-\mathrm{A}^{1} \Pi$ Angstrom

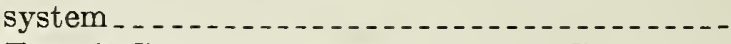

62. Franck-Condon factors for the $\mathrm{C}^{1} \Sigma^{+}-\mathrm{A}^{1} \Pi$

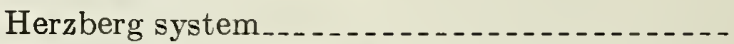

63. Franck-Condon factors, band strengths, $r$ centroids, and $I_{\infty}$ for the $b^{3} \Sigma^{+}-a^{3} \Pi$ Third Positive system . . . . . .

64. Franck-Condon factors for the $a^{\prime 3} \Sigma^{+}-a^{3} \Pi$

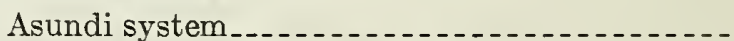

65. Franck-Condon factors for the $a^{3} \mathrm{I}-\mathrm{X}^{1} \Sigma^{+}$ Cameron system

66. Franck-Condon factors for the $b^{3} \Sigma^{+}-\mathrm{X}^{1} \Sigma^{+}$ Hopfield-Birge system . . . . . .

67. Franck-Condon factors for the $a^{\prime 3} \Sigma^{+}-\mathrm{X}^{1} \Sigma^{+}$ Hopfield-Birge system

68. Franck-Condon factors, $r$-centroids, and $I_{\infty}$ for the $d^{3} \Delta_{i}-a^{3} \Pi$ Triplet system

69. (a) Franck-Condon factors, $r$-centroids, and wavelengths for the $\mathrm{A}^{2} \Pi_{i}-\mathrm{X}^{2} \Sigma^{+}$Comet Tail system of $\mathrm{CO}^{+}$

(b) Smoothed band strengths and $I_{\infty}$ for the $\mathrm{A}^{2} \Pi_{i}-\mathrm{X}^{2} \Sigma+$ Comet Tail system of $\mathrm{CO}^{+}+--$

70. Franck-Condon factors, $r$-centroids, and wavelengths for the $\mathrm{B}^{2} \Sigma^{+}-\mathrm{X}^{2} \Sigma^{+}$First Negative system of $\mathrm{CO}^{+}$

71. Franck-Condon factors, $r$-centroids, and wavelengths for the $\mathrm{B}^{2} \Sigma^{+}-\mathrm{A}^{2} \Pi_{i}$ Baldet-Johnson system of $\mathrm{CO}^{+}$

72. Franck-Condon factors for ionizing transitions -

73. (a) Lifetimes, Einstein coefficients, and oscillator strengths.

(b) Einstein coefficients, absolute band strengths, and band oscillator strengths for the $\mathrm{A}^{2} \Pi_{i}-\mathrm{X}^{2} \Sigma^{+}$Comet Tail system of $\mathrm{CO}^{+}$

(c) Absolute f-values for the $\mathrm{A}^{1} \Pi-\mathrm{X}^{1}$ Fourth Positive system

74. Potential energy of the electronic states of $\mathrm{CO}$ and $\mathrm{CO}^{+}$

Page

66

66

66

66

68

\section{Figures}

2. Energy level diagram for $\mathrm{CO}$ and $\mathrm{CO}^{+}$ 


\title{
The Band Spectrum of Carbon Monoxide*
}

\author{
Paul H. Krupenie
}

\begin{abstract}
This is an exhaustive review of the literature and a critical compilation of the observed and predicted spectroscopic data on $\mathrm{CO}, \mathrm{CO}^{+}$, and $\mathrm{CO}^{2+}$ in the gas phase.
\end{abstract}

Key Words: Carbon monoxide, spectrum, review.

\section{Introduction}

There exists no unified compilation of spectroscopic data for diatomic molecules. The standard references in use, now incomplete and out of date, are the tables of molecular constants in Herzberg's book [99 ${ }^{1}$ and the more extensive compilations by Rosen et al., [213] which include band positions and perturbations. Rapid accumulation of new data has made apparent the need for a current critical review and more extensive compilation. This need has been partially met by recent tabulations of band head wavelengths by Pearse and Gaydon [187], and the compilation of these and other data for a select group of diatomic molecules of astrophysical interest by Wallace [266]. The only recent review known to the author which is devoted to a single molecule is that of Lofthus [153], "The Molecular Spectrum of Nitrogen."

The present work, first in a series on diatomic molecules, ${ }^{2}$ follows the approach of Lofthus and is devoted to a single molecule. This report includes a comprehensive review of the literature on the spectrum of $\mathrm{CO}, \mathrm{CO}^{+}$, and $\mathrm{CO}^{2+}$ in the gas phase, ${ }^{3}$ and a compilation of critically evaluated numerical data on band positions, molecular constants, energy levels, potential energy curves, and other molecular properties derived from the spectrum. Estimates of reliability are given where possible.

Papers from which the tabulated data have been extracted are discussed in the text of this report. Early data of presumably low precision have been included in the tabulations only where better or more recent data are not available. The references cited constitute a critical bibliography rather than an exhaustive one. The author will be

\footnotetext{
-Work supported in part by the National Acronautics and Space Admin. stration.

1 Figures in brackets indicate the litcrature references on page 78.

The second report in this series will be devoted to $\mathrm{O}_{2}$.

${ }^{3}$ Information on $\mathrm{CO}^{2+}$ is obtaincd from clectron impact.
}

indebted to readers who bring to his attention significant references not included in the bibliography.

No spectrograms are included in the report (but see table 1 for references with reproductions of spectra). However, R. W. Nicholls of the Department of Physics, York University Toronto, Ontario, Canada has forthcoming identification atlases for various band systems including several for carbon monoxide.

Discussions of infrared intensities, absorption coefficients, and line shapes and widths are omitted here; literature on these topics can be traced from references in the recent paper by Benedict et al., [18].

Carbon monoxide is an important molecule for the following reasons: (1) It is a product of combustion of organic molecules and plays an important role in the reactions of flames; (2) it appears as an impurity in many systems and its spectrum is readily excited, especially the strong $\mathrm{A}-\mathrm{X}$ Fourth Positive system in the UV; (3) it is a constituent of the solar chromosphere, stellar atmospheres, and comet tails $\left[\mathrm{CO}^{+}\right]$, [the increase in rocket and satellite observations will enhance its importance]; (4) because it is a light molecule its electronic structure is amenable to theoretical analysis; (5) it is isoelectronic with $\mathrm{N}_{2}$ which is important as the primary constituent in the earth's atmosphere; and (6) it can be obtained in pure form and is readily handled in the laboratory.

The spectrum of $\mathrm{CO}$ is dominated by three prominent band systems: $A^{1} \Pi-\mathrm{N}^{1} \Sigma^{+}$Fourth Positive system (2800-1140 $), b^{3} \Xi^{+}-a^{3} \Pi$ 'Third Positive system (3800-2600 $\AA$ ), and $B^{1} \Sigma^{+}-A^{1} \Pi$ Angstrom system $(6600-4100 \AA)$. The $A-\mathrm{I}$ system is the most pronounced and extensive, with more than 150 bands being observed. For $\mathrm{CO}^{+}$the $\mathrm{A}^{2} \Pi_{i}-\mathrm{X}^{2} \Xi^{+}$Comet 'Tail system ( 7200 $3080 \AA$ i) is the strongest. This system may be 
important in radiative heating of hypersonic vehicles at escape velocity or in the atmospheric fringe of the planet Venus (which contains $\mathrm{CO}_{2}$ ).

Study of the carbon monoxide spectrum in the visible region originated with the work of Angstrom and Thalén in 1875. The earliest band head measurements in the UV were made by Deslandres in 1888, and by Lyman shortly after the turn of this century. All of the early observations on CO have been cataloged in volumes V and VII of Kayser's Handbuch der Spektroscopie [127], but they can most easily be traced from an early review on the band spectra of CO by Birge [23].

The modern study of the spectrum of carbon monoxide began in 1926. In that year, Birge $[22,23]$ obtained the vibrational analysis of the $\mathrm{A}^{1} \Pi-\mathrm{X}^{1} \Sigma^{+}$Fourth Positive system, which, together with the correlation of emission and absorption measurements, enabled him to draw an energy level diagram for the observed electronic states. The impetus provided by the development of the quantum theory together with improved spectroscopic techniques resulted in extensive work in the 1930's on rotational analyses, and study of perturbations and predissociations. In the early 1940's, there appeared the first studies of Rydberg series, followed a decade later by the beginning of precise infrared vibration-rotation spectral measurements and microwave studies. Controversy of 30 years standing over the value of the dissociation energy of $\mathrm{CO}$ appears to have been convincingly settled by Douglas and Møller's re-examination [56] and review of the suspected predissociations of the $\mathrm{A}^{1} \Pi$ state.

The mid-1950's ushered in renewed interest in forbidden transitions and new states. Two bands previously thought to originate from high vibrational levels of the $a^{\prime 3} \Sigma^{+}$state (though questioned by Garg [68], Herzberg [99], and Gaydon [69]), have been indirectly found to originate from the $f^{3} \Sigma^{+}$state by more recent work of Herzberg and Hugo [101]. The $\mathrm{I}^{1} \Sigma$ - state, previously known only from its perturbations of the $A^{1} \Pi$ state, has been observed in forbidden transition to the $\mathrm{X}^{1} \Sigma^{+}$state. Complete details are not yet available.

Current effort is still centered more on the determination of energy levels (i.e., precise measurements of line positions) rather than on the more difficult intensity measurements.

\section{Electronic Structure of $\mathrm{CO}$ and $\mathrm{CO}^{+}$}

The early work of Mulliken [167, 168], largely intuitive and semiempirical, has laid the foundation for our understanding of the electronic structure and spectroscopic properties of carbon monoxide. Recent numerical calculations qualitatively account for a number of these features, though quantitative predictions are generally less reliable. The order of bound electronic states lying below $11 \mathrm{eV}$ has been correctly predicted, but not their numerical term values. Energies of some unobserved electronic states have been estimated. Calculated ionization potentials agree fairly well with experimental values. HartreeFock calculations question the experimentally determined polarity of the dipole moment. Theoretical prediction of the identity of the $E$ state as ${ }^{1} \mathrm{II}$ has been confirmed experimentally. Poor values are obtained for the ground state binding energy and potential energy curres of low lying states.

This section reviews what is known about the electronic structure of $\mathrm{CO}$ and $\mathrm{CO}^{+}$and briefly summarizes the results of numerical calculations. Elements of molecular orbital theory and definitions of terms used are given elsewhere [200, 250, 172]. Abbreviations used include: IP, ionization potential; MO, molecular orbital; LCAO, linear combination of atomic orbitals; CI, configuration interaction; SCF, self-consistent field; a.u., atomic units $(=27.210 \mathrm{eV})$.

\subsection{Electronic Structure}

The relative order of the molecular orbitals for $\mathrm{CO}$ is as follows $[46,171,168,109,173]$ :
(a) $\sigma 1 s<$
(b) $\mathrm{K}$
$\sigma^{*} 1 s<\sigma 2 s<$

$\begin{array}{cc}\sigma^{*} 2 s & < \\ y \sigma & \pi_{y} 2 p=\pi_{z} 2 p \\ 4 \sigma & w \pi \\ a . b . & 1 \pi \\ a . b .\end{array}$
$<\sigma 2 p$
$x \sigma$
$5 \sigma$
$3 \sigma$
$b$.
a.b.
$b$.
$<\quad \pi_{v}^{*} 2 p=\pi_{z}^{*} 2 p$
$v \pi$
$2 \pi$
a.b.

$<\sigma^{* 2} 2 p$
$u \sigma$
$6 \sigma$
$a . b$


Orbital designation (a) specifies the atomic origin of the MO's; designations (b) and (c) in current use, do not. The bonding character of the orbitals is specified above as: bonding, antibonding, or nonbonding. $1 \sigma$ and $2 \sigma$ orbitals virtually retain their purely atomic character and are of ten called $\mathrm{K}$ or inner orbitals. $3 \sigma$ is primarily $\mathrm{O}(2 s) ; 4 \sigma$ is a mixture of $\mathrm{C}(2 s)$ and $\mathrm{O}(2 p)$, though the actual ratio is a function of $r$. The $5 \sigma$ (slightly a.b.) and $1 \pi$ orbitals have similar energies and can be expected to have their order reversed at some value of $r$. Isoelectronic molecules have similar MO's and roughly similar sequence of MO's, as is the case for $\mathrm{CO}$ and $\mathrm{N}_{2}$ up to about $11 \mathrm{eV}$. At higher energies this similar order is destroyed.

The assignment of the electronic structure and dissociation products of a number of states of $\mathrm{CO}$ and $\mathrm{CO}^{+}$has been given by Mulliken [168]. The ground state configuration is

$$
\mathrm{X}^{1} \Sigma^{+}(1 \sigma)^{2}(2 \sigma)^{2}(3 \sigma)^{2}(4 \sigma)^{2}(1 \pi)^{4}(5 \sigma)^{2} .
$$

An examination of the ionization process from successively deeper lying orbitals shows why only three states are known for $\mathrm{CO}^{+}$. This is summarized below:

\begin{tabular}{c|l|r}
\hline \hline $\begin{array}{c}\text { Electron } \\
\text { ionized }\end{array}$ & $\begin{array}{l}\text { Ionic } \\
\text { state }\end{array}$ & I.P.(eV) ${ }^{4}$ \\
\hline & & \\
$5 \sigma$ & $\mathrm{X}^{2} \Sigma^{+}$ & 14.013 \\
$1 \pi$ & $\mathrm{A}^{2} \Pi_{i}$ & 16.544 \\
$4 \sigma$ & $\mathrm{B}^{2} \Sigma^{+}$ & 19.671 \\
$3 \sigma$ & ${ }^{2} \Sigma^{+}$ & $41 \pm 3$ \\
\hline
\end{tabular}

The ionization of the $3 \sigma$ electron has not been observed. There is some evidence for a state of $\mathrm{CO}^{+}$at $25.6 \mathrm{eV}( \pm 0.5 \mathrm{eV})$ from electron impact [151] and photoionization [270] studies. (This has been called the $\mathrm{C}^{2} \Sigma^{+}$state, but it does not correspond to the removal of a $3 \sigma$ electron.) Codling [42] has recently observed structure in the $500 \AA$ region which appears to include a Rydberg series whose convergence limit lies above $20 \mathrm{eV}$. By analogy with $\mathrm{N}_{2}^{+}$the electron configuration of this limiting state may result from a two-electron excitation. States of $\mathrm{CO}^{2+}$, detected at $41.8 \mathrm{eV}$ and $45.7 \mathrm{eV}$ in electron impret

- The three lowest IP lave been determined experimentally from Rydberg series limits and electronic speetra of $\mathrm{CO}^{+}$(see. 5.3). Only a calculated value is available for the IP of the $3 \sigma$ eleetron $[109,173]$. Long ago Mulliken [169] estimated this quantity as $32 \mathrm{eV}$. experiments [55, 263], have not been observed spectroscopically. Hurley and Maslen [111] have calculated the lower state energy to be $41.17 \mathrm{eV}$.

Ground state atoms $\mathrm{C}\left({ }^{3} \mathrm{P}\right)+\mathrm{O}\left({ }^{3} \mathrm{P}\right)$ give rise to 18 possible electronic states of $\mathrm{CO}$ (table 2). Only eight of these have been observed: $\mathrm{X}^{1} \Sigma^{+}$, $a^{3} \Pi, d^{3} \Delta_{i}, e^{3} \Sigma^{-}, a^{\prime 3} \Sigma^{+}, f^{3} \Sigma^{+}, A^{1} \Pi$, and $I^{1} \Sigma^{-}$; all the others are probably weakly bound or repulsive, though the ${ }^{1} \Delta$ state might be slightly stable [167]. However, a $\left({ }^{1} \Pi^{1} \Pi\right)$ or $\left({ }^{1} \Delta^{1} \Pi\right)$ perturbation at low $J$ in $\mathrm{A}^{1} \Pi, v=0,1$ at about $8 \mathrm{eV}$ can only come from a stable state $[44,71,216]$. Therefore, if real, these perturbations would most likely arise from one of the unobserved states. A possible $\left({ }^{1} \Delta^{3} \Delta\right)$ perturbation of the $d$ state indicates the presence of a ${ }^{1} \Delta$ state at about the same energy [137]. By analogy with $\mathrm{N}_{2}$ such a state would lie at about $9 \mathrm{eV}$.

Kaplan [126] has reported a state of unknown type at about $4.8 \mathrm{eV}$ above $\mathrm{X}$. The reality of a state of $\mathrm{CO}$ at this energy is, however, highly questionable [168, 99, 187].

For the higher energy excited molecular states beginning with $\mathrm{C}^{1} \Sigma^{+}$, at least one excited atom is required in the dissociation products, since the electronic energy alone exceeds the heat of dissociation into ground state atoms. In the case of singlet states of $\mathrm{CO}$ not formed from ground state atoms, both atoms must be excited. Table 1 is a summary of the known molecular constants and includes the assigned electron configuration and dissociation products where possible, for the observed electronic states. Table 2 gives the lowest lying configurations and possible molecular states, with the observed states indicated.

Mulliken [171] has recently compared the observed lower excited states of the $\pi^{3} \pi$ configuration with those predicted for $\mathrm{CO}$. The d-state of CO was proposed to be a case (b) $\pi^{3} \pi^{3} \Delta$ in disagreement with the earlier experimentally determined assignment as $d^{3} \Pi_{i}$. Carroll [40] experimentally confirmed Mulliken's assignment of the $d$ state as ${ }^{3} \Delta$, but definitely established it to be inverted. A $\pi^{3} \pi$ configuration gives rise to a ${ }^{3} \Delta_{r}$ state in the usual approximation. A theoretical interpretation of the anomalous multiplet splitting in the $d^{3} \Delta$ term has been given by Kíorács [137].

The $\pi^{3} \pi$ states are ${ }^{1} \beth^{+},\left[^{1} \Sigma^{-},{ }^{1}\right\lrcorner, a^{\prime 3} \Sigma^{+}, e^{3} \Sigma^{-}$, and $d^{3} \Delta_{2}$. Recent information [100, 23sa] indicates the I state lies at about $66000 \mathrm{~cm}^{-1}$, and the $e$ state at about $63000 \mathrm{~cm}^{-1}$. The $\left.{ }^{1}\right\rfloor$ state and ${ }^{1} \mathrm{I}^{+}$remain unobserved. 


\subsection{Numerical Calculations}

\section{a. Semiempirical}

Semiempirical studies have provided a qualitative understanding of several properties of the carbon monoxide molecule. The triple bond for the ground state has been accounted for by Sahni [217], Mulliken [170], and Linnett [152]. (See also ref. [154].) Moffitt [164] has correlated the decrease in bond length for states of $\mathrm{CO}$ and $\mathrm{CO}^{+}$with increase in $\pi$-bond order. Sahni [217], with neglect of $1 s$ electrons and the use of many approximate integrals, has derived individual and total orbital electron densities which are shown in contour diagrams. The lowest ionization of $\mathrm{CO}$, that is, removal of an almost nonbonding electron from the $5 \sigma$ orbital, has been shown loosely equivalent to removal of an electron from the carbon atom $[217,170,152]$. Indeed, the dissociation products of the ground state of $\mathrm{CO}^{+}$are $\mathrm{C}^{+}+\mathrm{O}$.

\section{b. Single Configuration}

Several calculations for $\mathrm{CO}$ have been made using single configuration LCAO-MO-SCF wave functions. Ground state energies have been calculated by Ransil [199, 200] and Hurley [110] using functions constructed from minimal basis sets. Merryman et al. [159], and Brion and Moser [29] have calculated excitation energies for a number of states from similar functions. For states lying above $9 \mathrm{eV}$ the calculated energies are too high. To describe such states it is necessary to include more than one configuration or to introduce atomic orbitals of higher quantum number. LefebvreBrion et al. [146], have compared the vertical excitation energies calculated from SCF functions built from extended basis sets. Calculated values agree fairly well with experiment, even for states lying above $11 \mathrm{eV}$.

The most accurate wave functions for the ground state of $\mathrm{CO}$ have recently been reported by Wahl and Huo [265] and Huo [109]. Huo has calculated single determinantal SCF wave functions from two extended basis sets, including one built by extending the basis set necessary for obtaining accurate Hartree-Fock functions for the atoms. 'The calculated value of the total molecular energy is -112.7877 a.u. This result was obtained from a minimal basis set supplemented by $1 s_{0}^{\prime}, 1 s_{c}^{\prime}, 2 s_{0}^{\prime}$, $2 s_{c}^{\prime}, 2 p \pi_{0}^{\prime}, 2 p \pi_{c}^{\prime}, 2 p \sigma_{0}^{\prime}, 2 p \sigma_{c}^{\prime}, 3 d \sigma_{0}, \quad 3 d \sigma_{c}, 3 d \pi_{0}$, $3 d \pi_{c}, 2 p \pi_{0}^{\prime \prime}, 4 f \pi_{c}, 3 s_{0}$, and $3 s_{c}$. Expectation values of several one-electron operators are given as well as contour diagrams for orbital and total charge densities [109(b)].

Expectation values of a number of one-electron operators were calculated, including the dipole moment whose polarity was determined as $\mathrm{C}^{+} \mathrm{O}^{-}$, in disagreement with the reverse assignment given by Rosenblum, Nethercot, and Townes [215] from microwave experiments. Huo [109] and Nesbet [173] have independently concluded that more accurate SCF functions should not change the sign of the dipole moment, and that the polarity should be considered undetermined.

Nesbet [173] has calculated SCF functions for the ground state of $\mathrm{CO}$ using a double exponential basis set with optimized exponents for each occupied atomic orbital. This basis set appreciably lowers the total energy but makes only a small change in the binding energy from that using a smaller basis set. The addition of $d \sigma$ and $d \pi$ orbitals makes only a small change in the total energy but makes a larger improvement in the binding energy.

\section{c. Configuration Interaction}

Configuration interaction has been used to calculate the correlation energy [experimental total energy-Hartree-Fock energy]. Combined use of extended basis sets and CI results in improved values of excited state energies and gives lower calculated total energies. Lefebvre-Brion et al. [144], have found that use of a basis set which includes functions from the M shell ( $3 s$ and $3 p$ ) with Slater exponents improves the calculated vertical excitation energies more than does doubling the number of $\mathrm{L}$ shell functions. By using orbitals constructed from a minimal basis set and including CI they have also [145] calculated potential energy curves for a number of states. These include repulsive ${ }^{3} \Pi$ and ${ }^{1} \Pi$ states which lie at 11.5 and $12 \mathrm{eV}$, respectively. The latter value agrees with the vertical excitation energy of the $F$ state and suggests that the $F$ state is not ${ }^{1} \Pi$. [See also ref. 146.] The fragmentary data in section 5 suggest that the $F$ state may be triplet. Lefebvre-Brion [147] predicts the state to be ${ }^{1} \Sigma^{+}$ (table 1).

Fraga and Ransil [66] have calculated the molecular energy and dipole moment for the ground state of CO using a limited CI with minimal SCF wave functions. Even with 14 configurations (having the same symmetry as the ground state) 
$\mathrm{E}_{\text {calo }}-\mathrm{E}_{\mathrm{obs}}$ is about $27 \mathrm{eV}$. These results indicate the need [66] to include MO's with different $\lambda$ values and atomic orbitals of different $l$ values from those utilized in the ground state.

\subsection{Rydberg States and Rydberg Series}

The observed Rydberg states in CO (table 1 supplement) are most probably singlet, and either II or $\Sigma^{+}$, since they have all been detected in absorption series. There may exist others which do not combine with the ground state and could be observed in emission, but to date none have been reported. The electronic structure of the states considered is given below:

\begin{tabular}{c|c|c}
\hline \hline $\begin{array}{c}\text { Configu- } \\
\text { ration }\end{array}$ & Possible Rydberg states & $\begin{array}{c}\text { Convergence } \\
\text { limit }\left[\mathrm{CO}^{+}\right]\end{array}$ \\
\hline$\sigma(\mathrm{R} \sigma)$ & ${ }^{1} \Sigma^{+},{ }^{3} \Sigma^{+}$ & $\mathrm{X}, \mathrm{B}$ \\
$\sigma(\mathrm{R} \pi)$ & ${ }^{1} \Pi,{ }^{3} \Pi$ \\
$\pi^{3}(\mathrm{R} \sigma)$ & ${ }^{1} \Pi,{ }^{3} \Pi_{i}$ & $\mathrm{~A}$ \\
$\pi^{3}(\mathrm{R} \pi)$ & ${ }^{1} \Sigma^{+},{ }^{1} \Sigma^{-},{ }^{1} \Delta,{ }^{3} \Sigma^{+},{ }^{3} \Sigma^{-},{ }^{3} \Delta$ & \\
\hline
\end{tabular}

For the most likely Rydberg orbitals $n p \pi$ or $n p \sigma$, the most likely states are $\Pi$ or $\Sigma$. Huber [106] believes that the states converging to the $\mathrm{X}$ and $\mathrm{B}$ states of $\mathrm{CO}^{+}$are ${ }^{1} \Sigma^{+}$and the $\alpha$ states converging to $A^{2} \Pi_{i}$ are ${ }^{1} \Pi$, all having Rydberg. orbitals $n p \sigma$. Tanaka [254] has speculated that the sharp $\beta$ series is composed of ${ }^{1} \Sigma^{+}$states and the diffuse $\beta$ states are ${ }^{1} \Pi$. With present know- ledge it is not possible to specify uniquely their symmetry types.

Rydberg states with orbital $n$ dissociate into atoms at least one of which is configurationally excited (i.e., having principal quantum number greater than in its ground state, $(n-1) \geq 3)$. Huber [106] sugges ts that the dissociation products of series converging to states $\mathrm{X}$ and $\mathrm{A}$ are $\mathrm{C}(n-1) \mathrm{s}\left({ }^{3} \mathrm{P}^{0}\right)+\mathrm{O}\left({ }^{3} \mathrm{P}\right)$, and states converging to $\mathrm{B}$ have products $\mathrm{C}(n-1) s\left({ }^{1} \mathrm{P}^{0}\right)+\mathrm{O}\left({ }^{1} \mathrm{D}\right)$.

Lefebvre-Brion, Moser, and Nesbet [147] have recently calculated the energies of Rydberg levels of $\mathrm{CO}$ which are of symmetry type ${ }^{1,3} \Sigma^{+}$ and ${ }^{1,3} \Pi$ and lie below the first ionization potential. The calculated energies fall within $0.2 \mathrm{eV}$ of the observed values for the $\mathrm{B}^{1} \Sigma^{+}, \mathrm{C}^{1} \Sigma^{+}, b^{3} \Sigma^{+}$, and $c^{3} \Sigma^{+}$states which probably belong to Rydberg series converging to the ground state of $\mathrm{CO}^{+}$. The configuration interaction functions used were built from LCAO-MO-SCF orbitals used previously $[146,148]$ for calculations on the ground state of $\mathrm{CO}$, but with extended basis sets.

The deviations between these calculated energies and experiment indicate that the $\mathrm{E}$ state is ${ }^{1} \mathrm{II}$, and the $\mathrm{F}$ state may be ${ }^{1} \Sigma^{+}$. The $\mathrm{E}$ state assignment has been confirmed experimentally by Tilford et al. [257]; the tentative assignment of the $F$ state as ${ }^{1} \Sigma^{+}$is untested. (See sec. 5.2f and table 1, footnote $\mathrm{F}\left({ }^{1} \Sigma^{+}\right)$.) No ${ }^{3} \Pi$ Rydberg levels of $\mathrm{CO}$ have been observed to date. Codling [42] has recently observed Rydberg series converging to what may be a new state of $\mathrm{CO}^{+}$above $20 \mathrm{eV}$, but the symmetry and type of Rydberg levels have not been identified as yet.

\section{Electronic Spectrum of $\mathrm{CO}$ and $\mathrm{CO}^{+}$}

The spectral region $8600-600 \AA$ includes all the presently known electronic transitions of $\mathrm{CO}$ and $\mathrm{CO}^{+}$. Intense systems span most of the region between 7500-1100 ̊. From $3800 \AA$ to longer wavelengths are found the B-A Angstrom bands and the $d-a$ Triplet system, the latter system composed of many bands, only four of which have had rotational analysis. Between 7.200-3000 $\AA$ is found the $\mathrm{A}-\mathrm{X}$ Comet Tail system of $\mathrm{CO}^{+}$, the most prominent system of the ion. From 3800$2600 \AA$ is the $b-a$ Third Positive system which is most important because of the most violent pelturbations and predissociation of the $b$ state. 'The
A-X Fourth Positive system dominates the region between 2800-1140 $\AA$, though the shorter warelength bands are rather weak. Below $1200 \AA$ are found, in general, weak systems, incompletely studied, for which no rotational analysis is arailable. In total 29 transitions are found among the 23 known states of $\mathrm{CO}$. Three states of $\mathrm{CO}^{+}$ partake in three observed transitions. Detaits of the observed transitions are discussed below, including the determination of vibrational and rotational constants, multiplet splitting, and coupling cases of the electronic states. The critically evaluated data pertaining to these transitions have been tabulated in section 12. 


\section{1. $\mathrm{A}^{1} \Pi-\mathrm{X}^{1} \Sigma^{+}$Fourth Positive System (2800-1140 ̊) R}

The $\mathrm{A}^{1} \Pi-\mathrm{X}^{1} \Sigma^{+}$Fourth Positive system, originally attributed to carbon, is the most prominent system of carbon monoxide in the ultraviolet and vacuum UV regions. The single-headed reddegraded bands of this allowed transition are about a thousand times stronger than those due to forbidden transitions which they overlap. Wavelengths of more than 150 emission bands which have been observed in the region 2800-1280 $\AA$ are assembled in table 3a. Included among these are early measurements by Lyman and Deslandres of 30 band heads whose vibrational assignments are due to Birge [23], and which have not been reported by anyone else. Band origins for this system are given in table 4 . Rotational constants for the $\mathrm{X}$ and $\mathrm{A}$ states are given in tables 36 and 37 , respectively. Positions of a number of unidentified bands have been assembled in table $35 \mathrm{a}$. These have been observed together with the $A-X$ system and possibly belong to it.

Attention is focused on the $\mathrm{A}-\mathrm{X}$ system by the numerous perturbations of the $A^{1} \Pi$ state, a detailed discussion of which is given in section 4.1. The recently observed $\mathrm{I}^{1} \Sigma^{-}$state was first identified from its interaction with the A state. Several predissociations of the A state have been reported which have led to conflicting values of the dissociation energy of CO. Re-examination of these by Douglas and Møller [56] has shown that none of them are real.

The work before 1926, including the earliest band head measurements by Deslandres and Lyman, was discussed and analyzed for the first time by Birge [23]. All observations prior to that date were in emission, except for Leifson's [149] observation of the strong $v^{\prime \prime}=0$ progression (with $v^{\prime}=0$ to 11 ) in absorption. The vibrational analysis of this system [23,22] and the correlation of Lyman's emission bands with the absorption bands of Leifson showed that they were due to neutral $\mathrm{CO}$. Thus an energy level diagram could be drawn with the lower state of this system established as the ground state.

Estey [60] remeasured part of the A-X system in the region 2800-1970 $\AA$, and observed 16 new bands which involve high vibrational levels of the ground state. His source was a low pressure discharge, viewed with a quartz spectrograph having dispersion $3.91 \AA / \mathrm{mm}-1.21 \AA / \mathrm{mm}$. Each band clearly shows a single $\mathrm{P}, \mathrm{Q}$, and $\mathrm{R}$ branch as is expected for a ${ }^{1} \Pi^{1} \Sigma$ transition. Less extensive band head measurements by Wolter [277] agree with those of Estey to within $0.1 \AA$.

Headrick and Fox [90] measured bands in the region 2170-1280 $\AA$, using a 1-m focal length vacuum spectrograph. Several new bands were reported, mostly at shorter wavelength. The source was a low voltage are in $\mathrm{CO}$ at $0.5 \mathrm{~mm}$ pressure with a trace of hydrogen. Wavelengths obtained from the use of two different standards agreed to within $0.2 \AA$, and are close to the values of Estey [60] where they overlap near $2000 \AA$. Dispersion was $17.4 \AA / \mathrm{mm}$. Intensities, originally given as densitometer readings, have been converted in this report to a scale with the maximum reading taken as 10 . In table $3 \mathrm{a}$, for the bands above $2000 \AA$, the intensities are those given primarily by Estey [60]; below $2000 \AA$, the intensities are those of Headrick and Fox [90]. Several strong unidentified bands at short wavelengths have been included in table 35a.

Read [209] has given the vibrational analysis of 65 heads, observed in a hollow cathode discharge in flowing $\mathrm{CO}_{2}$, and viewed with a $2-\mathrm{m}$ focal length vacuum spectrograph with dispersion of $4.2 \AA / \mathrm{mm}$. In addition, he resolved the rotational structure for 12 bands, but details only of the 1-1 and 4-10 bands have been published [209b]. This was the first rotational analysis of bands involving the ground state of CO. $\Lambda$-doubling was found to be negligible, as had been determined from earlier studies of the $\mathrm{B}^{1} \Sigma-\mathrm{A}^{1} \Pi$ Angstrom bands.

A formula was obtained which fit the newly reported band heads as well as those of Estey at longer wavelength:

$$
\begin{aligned}
\sigma_{H}\left(v^{\prime}-v^{\prime \prime}\right)= & 64756.3+\left(1497.49 v^{\prime}-17.1841 v^{\prime 2}\right) \\
& -\left(2155.61 v^{\prime \prime}-13.2843 v^{\prime \prime 2}\right. \\
& \left.+0.012 v^{\prime 3}\right)-0.0804 v^{\prime} v^{\prime \prime} .
\end{aligned}
$$

The derived ribrational constants are very close to the best values listed in table 1 .

The papers of Estey [60], Headrick and Fox [90], and Read [209] include almost all of the emission band head measurements of the $\mathrm{A}-\mathrm{X}$ system. Shortly after their publication there began to appear extensive rotational analyses, mostly by Schmid and Gerö.

In a Geissler discharge in $\mathrm{CO}_{2}$, Gerö [73] observed a series of bands in the region 2700-1950 ̊. Numerous perturbations were easily recognized in all branches. Using a 6.5-m focal length grating 
with dispersion of $1.2 \AA / \mathrm{mm}$, he obtained the rotational structure of the $4-11,5-13,6-15,7-16$, 8-17, 9-19, 10-20, and 10-21 bands. Gerö further reported [74] rotational structure for the 9-18, 11-20, 12-22, and 13-24 bands, and, in addition, gave a Deslandres table of 84 band origins calculated from the band heads with a positive correction of $\frac{\left(\mathrm{B}^{\prime}+\mathrm{B}^{\prime \prime}\right)}{4\left(\mathrm{~B}^{\prime}-\mathrm{B}^{\prime \prime}\right)}$, making use of previous data [209, 73].

Table 4 gives an extensive list of band origins which includes several additions of low accuracy which supplement the Deslandres table of Herzberg [99, p. 156-7]. Herzberg's table is mainly that of Gerö [74] with slight modifications and numerous additions of low accuracy. The A-X system origin is at $64746.5 \mathrm{~cm}^{-1}$ [209].

The heads of the 14-23, 15-25, and 16-25 bands listed by Estey [60] deviate by $+13.2,+14.8$, and $+21.7 \mathrm{~cm}^{-1}$, respectively, from Read's formula. Gerö [74] believed that the appearance of these bands was due to accidental accumulation of lines, where strong overlapping is observed. The first of these heads lies close to a band reported by Kaplan [125] at $2518 \AA$, and the third lies close to a band of the $3 \mathrm{~A}$ system $(c-a)$. Gerö concluded from this evidence that the $v=13$ level of the $A$ state was the last before dissociation. However, Tanaka, Jursa, and LeBlanc [255] have since observed the A state to $v=20$.

Schmid and Gerö [228] reported the measurement and rotational analysis of many bands by Kelemen [in a dissertation], including 1-8, 2-9, $2-10,3-9,3-10,3-12,4-10,4-12,4-13,5-11,5-12$, $5-14,6-13,6-14,7-14,7-15,7-17,8-16,8-18$, $8-19,9-17,9-20$, and 10-19. From these data and those of Read [209] and Gerö [73, 74], they obtained formulas for the rotational constants for both the $\mathrm{X}^{1} \Sigma^{+}$and $\mathrm{A}^{1} \Pi$ states. Schmid and Gerö [228] suggested that Read should have included a higher power of $J$ in his expression for $\mathrm{F}_{0}(\mathrm{~J})$, which would have resulted in slightly larger $B_{0}$ values. The calculation of $B_{v}$ for the strongly perturbed A state follows the method of Gerö [71]. Least squares fitting to the observed data gives for $\mathrm{A}^{1} \Pi: \mathrm{B}_{0}=1.6116-0.02229(v+1 / 2)-$ $0.000105(v+1 / 2)^{2}$ (table 1). Brons' [31] rotational analysis of the $10-19,10-20$, and $10-21$ bands gives $\mathrm{B}_{10}^{\prime}, \mathrm{B}_{19}^{\prime \prime}, \mathrm{B}_{20}^{\prime \prime}$, and $\mathrm{B}_{21}^{\prime \prime}$, slightly smaller than the values of Schmid and Gerö [22S].

Tschulanowsky and Stepanow [262] have given the rotational analysis of the $3-8,3-7,2-8,2-7$,
2-6, 1-6 and $1-5$ bands, including the determination of rotational constants and a discussion of various perturbations of the $\Lambda$ state. 'Twentyfour bands were observed with a dispersion of $8.4 \AA / \mathrm{mm}$ (with resolution of lines separated by $0.07 \AA$ ), and details of the three best are given. Branches were observed up to $\mathrm{J}$ values of 35 . The three best bands for analysis were $3-8,2-7$, and $1-6$. The $R$ branches were measured very inaccurately and their details are not given.

Onaka [182] has looked at the 6-13 and 6-14 bands with a vacuum echelle spectrograph of high resolution $(\sim 170,000)$ and high dispersion (0.30-0.26 $\AA / \mathrm{mm})$. Earlier attempts at analysis of the lower rotational levels in $A^{1} \Pi, v=6$ was prevented by the complicated structure due to overlapping of the $v=6$ level by levels of the $d^{3} \Delta_{i}$ and $a^{\prime 3} \Sigma^{+}$states. Several perturbations were found (see sec. 4.1). The lines of the 6-14 band are not given. A formula for the rational structure of the $v=6$ level is given by Onaka from an unpublished work on the Fourth Positive system:

$$
\mathrm{T}_{v=6}\left(\mathrm{~A}^{1} \Pi\right)=74197.44+1.4616 \mathrm{~J}(\mathrm{~J}+1) .
$$

Tanaka, Jursa, and LeBlanc [255] reported on the absorption spectrum of the Fourth Positive system in the vacuum UV for the first time since the pioneer work of Leifson [149], and Hopfield and Birge [104]. Using rare gas continua as sources, Tanaka et al., observed new bands and extended known bands to shorter wavelengths, all under low resolution, covering the spectral region of $1177-1544 \AA$. The $\left(v^{\prime}-0\right)$ progression was observed for $v^{\prime}=0$ to 20 ; also the $\left(v^{\prime}-1\right)$ progression for $v^{\prime}=0$ to 13 . In addition, the isotopic $\mathrm{C}^{13} \mathrm{O}^{16}$ band progiession $\left(v^{\prime}-0\right)$ was obserred up to $v^{\prime}=17$ (not including 16). Tanaka et al., used pure $\mathrm{CO}$ in an 8 -cm long absorption cell, at pressures of 0.01 to $600 \mathrm{~mm}$ of $\mathrm{Hg}$. The spectrograph used was a $2-\mathrm{m}$ focal leng th normal incidence type with a dispersion of about $8 \AA / \mathrm{mm}$. The absorption band positions are given in table $3 \mathrm{~b}$; the observed isotopic bands of $\mathrm{C}^{13} \mathrm{O}^{16}$ are giren in table 5. Several unidentified bands which were found are given in table $35 \mathrm{~b}$.

McCulloh [157a] and McCulloh and Glockler [157b] first studied emission by $\mathrm{C}^{13} \mathrm{O}^{16}$ in tho positive column of an uncondensed discharge nt low current density in $\mathrm{CO}_{2}$ in tho spectral region of $1900-6500 \AA$. 'They reported ribrational analysis of 35 bands of the Fourth Positive system 
and rotational analysis of bands in the $\left(0-v^{\prime \prime}\right)$ progression of the Angstrom system $\left(B^{1} \Sigma^{+}-A^{1} \Pi\right)$. Band origins were obtained from the head measurements by using rotational constants of Schmid and Gerö [228] and the equations for the isotope effect. Low resolution precluded rotational analysis. The observed emission heads and origins of the isotopic $\mathrm{A}-\mathrm{X}$ bands are given in table 5a.

McCulloh and Glockler [157b] believed that Read's [209] vibrational formula for the ground state was good for higher vibrational levels of $\mathrm{C}^{12} \mathrm{O}^{16}$. However, they assumed that his results were unduly influenced by errors in the short wavelength heads due to poor standards, and were not consistent with more recent infrared measurements. Hence, they fitted molecular constants for the normal and isotopic species which represented both Read's data and the infrared data of Lagemann et al., [139]. Slightly different constants have recently been calculated by Benedict [17] to fit the most recent infrared data as well as that for the A-X system. (See table 1.) Vibrational constants for the $\mathrm{A}^{1} \Pi$ state of $\mathrm{C}^{13} \mathrm{O}^{16}$ were obtained [157] from Read's values for the normal isotope and the ratio of reduced masses. These are included in table 37.

High dispersion absorption spectra in the vacuum UV region have recently been photographed by Herzberg and Bass [100], and by Wilkinson and Tilford [276]. Several previously unobserved transitions are included in these studies, as well as bands of the $v^{\prime \prime}=0$ progression of the $A-\mathrm{X}$ system up to $v^{\prime}=20$, some bands of the $v^{\prime \prime}=1$ progression, and also isotopic $\left(\mathrm{C}^{13} \mathrm{O}^{16}\right)$ $\mathrm{A}-\mathrm{X}$ bands. The rotational analyses of these bands should provide definitive values of the rotational constants for the $A^{1} \Pi$ state and precise vibrational quanta for this state [238a].

\section{2. $B^{1} \Sigma^{+}-A^{1} \Pi$ Angstrom System (6620-4120 A) V}

In 1875, Angstrom and Thalén [see ref. 23] ${ }^{5}$ observed a group of bands which became known as the Second Positive system of carbon. Fifty years later, analysis by Birge [23] showed that this system, which now bears the name of Angstrom, arose from the allowed transition $\mathrm{B}^{1} \Sigma^{+}-\mathrm{A}^{1} \Pi$ in carbon monoxide. This system has been a fertile ground for study because of the numerous

\footnotetext{
- The data of Angstrom and Thalén, as well as fine structure obscrvations of Loos and head measurements by Watts and Wilkinson at the turn of the century, are also listed on p. 277 of Vol. V of Kayser's "Handbuch der Spectroscopie" [127a].
}

perturbations and suspected predissociations of the A state. These single-headed violet-degraded bands have also been observed for the isotope $\mathrm{C}^{13} \mathrm{O}^{16}$. Band heads and origins for the normal isotope are given in table 6 ; the isotopic $\left(\mathrm{C}^{13} \mathrm{O}^{16}\right)$ heads and origins are given in table 7 . Rotational constants for the $\mathrm{A}$ and $\mathrm{B}$ states are given in tables 37 and 38 , respectively.

Included among the original observations were weak bands at $4301 \AA$ and $4581 \AA$ which were later identified [23] as possibly belonging to the $v^{\prime}=2$ progression but which lay to warelengths longer by more than $10 \AA$ from the expected head positions. Johnson and Asundi [121] believed that these two bands belonged to another system. Only one other band with $v^{\prime}=2$ has been reported [255], but its identification is also uncertain since the B state is predissociated in levels $v=0$ and 1 and presumably also in $v=2$ at $\mathrm{J}=0$. Another uncertain band, included as $0-6$ by Birge [23], lies about $8 \AA$ to longer wavelength from its expected position. A band observed by McLennan, Smith, and Peters [158] at $7246 \AA$, has not been reported elsewhere.

The first rotational quantum analysis of the $0-1,0-2,0-3$, and $1-1$ bands was made by Hulthén [108]. The $5610 \AA$ band was the strongest observed, and incorrectly assigned as the 2-2 band. The correct vibrational quantum assignments are obtained by reducing Hulthén's $v^{\prime}$ by 2 and raising his $v^{\prime \prime}$ by 1 . Bands were also observed at $6078 \AA, 6622 \AA$, and $4511 \AA$, but the structure of these could not be determined at that time.

Jasse [115] has given the rotational analysis for the $0-0$ and $1-0$ bands. His source was a high voltage electric discharge in $\mathrm{CO}_{2}$ at low pressure. Spectra were taken using a grating spectrograph of $3.25-\mathrm{m}$ focal length; claimed precision is \pm 0.01 A. Hulthén [108] and Jasse [115] believed that the structure of the $0-0$ and 1-0 bands was more complicated than the others of this system, having two additional branches. Birge [23] believed that the additional branches were probably spurious, while Johnson and Asundi [121] thought that the additional branches might originate from overlapping lines of $\mathrm{C}_{2}$. Later, more accurate work by Rosenthal and Jenkins [216], and Schmid and Gerö [224] indicated that perturbations of $A^{1} \Pi, v=0$ were responsible for the confused structure of the $0-0$ and $1-0$ bands, resulting in displacement of the band heads from their expected positions. 
Johnson and Asundi [121] observed the 0-0 and $0-1$ bands in the first order of a 21-ft focal length grating spectrograph (supplanting Jasse's measurements), and re-evaluated constants from Hulthén's data [108]. 'They also gave the first preliminary evaluation of the fine structure of the 0-4 and 0-5 bands, with details given by Asundi [5]. Referring to Jasse's analysis of the $4511 \AA$ band, they considered the lines at $22172.3 \mathrm{~cm}^{-1}$ and $22175.7 \mathrm{~cm}^{-1}$ as extraneous to the band. Also they relabeled the line at $22171.8 \mathrm{~cm}^{-1}$ as Q (1).

New measurements of fine structure of the $0-0$, $0-1,0-2$, and $0-3$ bands were made by Rosenthal and Jenkins [216] primarily to study perturbations, though the results are not completely reported in this work. (See sec. 4 on Perturbations.) It was found that Jasse, and Johnson and Asundi, did not correctly identify $P$ and $R$ lines above $J=S$ and $Q$ lines above $J=10$ because of the large increase in $\Lambda$-doubling in the region of a perturbation. [See fig. 1 of ref. 216-the difference between the $\mathrm{R}$ and $\mathrm{Q}$ deviation curves.] Corrections to Jasse's assigments for several lines of the $0-0$ band were made [216].

Coster and Brons [44] have given the rotational analysis of the $0-0,0-1,0-2,0-3$, and $0-4$ bands in order to interpret the perturbations of the $v=0,1,2,3$, and 4 levels of the $\mathrm{A}^{1} \Pi$ state. Their discharge tube produced strong Angstrom bands relative to the background of $\mathrm{CO}_{2}$ bands. A value of $\sigma_{\mathrm{H}}(0-0)$ for the system of $22163.1 \mathrm{~cm}^{-1}$ was obtained. (Herzberg [99] lists $22171.3 \mathrm{~cm}^{-1}$.) (See also sec. 4.1.)

Schmid and Gerö [224], from observations in second and third order using a $21-\mathrm{ft}$ focal length grating spectrograph, gave improved measurements of the $0-0,0-1,1-0$, and $1-1$ bands. They obtained rotational constants for the $\mathrm{B}^{1} \Sigma^{+}$state, and in addition, $\Delta \mathrm{G}\left(\frac{1}{2}\right)=2082.07 \mathrm{~cm}^{-1}$.

McCulloh [157a] and McCulloh and Glockler [157b] have reported the B-A Angstrom bands due to $\mathrm{C}^{13} \mathrm{O}^{16}$ emission in the positive column of an uncondensed discharge. The perturbations in the $A^{1} \Pi$ state are discussed at some length [157a] (see sec. 4.1) and details are given of the rotational analysis of the $\left(0-v^{\prime \prime}\right)$ progression including the $0-1,0-2,0-3,0-4$, and $0-5$ bands, and the $1-1$ band. The $\mathrm{C}^{13} \mathrm{O}^{16}, 0-0$ head lies to the red of the $\mathrm{C}^{12} \mathrm{O}^{16}$ head. Overlapping by Third Positive bands $\left(b^{3} \Sigma^{+}-a^{3} \Pi\right)$ prevented rotational analysis of the 0-5 isotopic band. Overlapping of regular and isotopic bands required $0-1$ branches to be identified by a method analogous to the LoomisWood method (see pp. 191-2 in Herzberg [99]). For the 0-2 band there is almost complete superposition of the $P$ and $R$ branches such that $R(J) \sim$ $P(J+10)$. The region beyond the tail of this band and also the $0-3$ band is quite free from the general background caused by $\mathrm{CO}_{2}$ bands. 'Therefore, these bands are useful when searching for predissociation in the $\mathrm{B}^{1} \Sigma^{+}$state. The $0-4$ band has a near superposition of the $P$ and $R$ branches.

Since only one $v^{\prime \prime}$ progression was analyzed no information can be obtained about the vibrational constants of the $\mathrm{B}^{1} \Sigma^{+}$state. (See Schmid and Gerö [224].) Values for $\omega_{e}$ and $\omega_{e} x_{e}$ for the isotopes $\mathrm{C}^{12} \mathrm{O}^{16}$ and $\mathrm{C}^{13} \mathrm{O}^{16}$ were approximated from a study of the isotope effect. The abnormally large value of $\omega_{e} x_{e}$ for the $B$ state suggests probable rapid convergence of levels. (See table $9 \mathrm{a}$ and footnote $\mathrm{B}^{1} \Sigma^{+}$to table 1.)

Douglas and Møller [56] have photographed under high dispersion and analyzed the $0-1,1-1$, and 0-2 Angstrom bands of $\mathrm{C}^{13} \mathrm{O}^{16}$. The bands were observed in a discharge through $\mathrm{O}_{2}$ in a tube containing carbon, using a $21-\mathrm{ft}$ focal length grating spectrograph with a reciprocal dispersion of $2.5 \AA / \mathrm{mm}$. 'Their primary aim was an examination of reported predissociations of the $\mathrm{C}^{12} \mathrm{O}^{16}$ and $\mathrm{C}^{13} \mathrm{O}^{16}$ molecules (see sec. 5.2).

\section{3. $\mathrm{C}^{1} \Sigma^{+}-\mathrm{A}^{1} \Pi$ II Herzberg System (5710-3680 A) V}

The $\mathrm{C}^{1} \Sigma^{+}-\mathrm{A}^{1} \Pi$ Herzberg system, which partly overlaps the B-A Angstrom system, consists of eight observed bands of the $v^{\prime}=0$ progression. No bands of this system have been observed with $v^{\prime}>$ 0 , but the level C, $v=1$ has recently been observed in the $\mathrm{C}-\mathrm{X}$ system [276]. This suggests that there is either predissociation for $v^{\prime}>1$, or that a weak transition is expected from the FranckCondon principle. Table 8 lists the heads and origins of the $\mathrm{C}-\mathrm{A}$ system. The rotational constants for the $\mathrm{C}^{1} \Sigma^{+}$state are give in table 39 and those for the $A^{1} \Pi$ state in table 37 . Three bands of this system were first observed by Duffendack and Fox [5S] who thought that the new bands belonged to the Angstrom system because of their similar structure. Herzberg [97] obserred eight bands and recognized these as belonging to a new systen whose upper state he incorrectly designated as ${ }^{1} \Delta$, by analogy with the Grotrian diagram for the Mig atom, as was done at that 
time, following Mulliken. The new level lay between the $\mathrm{B}^{1} \Sigma^{+}$and $c^{3} \Sigma^{+}$states.

Asundi [3] and Asundi and Johnson [121] observed the $0-1,0-2$, and $0-3$ bands using a 21 -ft focal length grating spectrograph in first order (wavelengths of the stronger lines are believed accurate to within $0.01 \AA$ ). Fine structure analysis showed the $\mathrm{C}$ state to be ${ }^{1} \Sigma$. The $\mathrm{Q}$ branches were reported to be twice as strong as the $P$ and $R$ branches. For the $A^{1} \Pi$ state there is no combination defect up to $J=17$ (limit of observations here), indicating no appreciable $\Lambda$-doubling to high rotational quantum numbers. This is so for $v^{\prime \prime}=1$ to 5 , not for $v^{\prime \prime}=0$, for which combination defects occur from almost the lowest J values. [See ref. 224.] For the 0-0 band, only the calculated position is given, because of confusion with a band associated with the Third Positive system of $\mathrm{CO}\left(b^{3} \Sigma^{+}-a^{3} \Pi\right) . \quad \mathrm{A}$ band reported by Deslandres at $3893 \AA$ which Wolter [277] did not observe is the $0-1$ band of the C-A system.

Schmid and Gerö [224] observed the 0-0, 0-1, $0-2,0-3$, and $0-4$ bands of the $\mathrm{C}-\mathrm{A}$ system. From the rotational analysis, a value of $\mathrm{B}_{0}{ }^{\prime}$ of $1.9422 \mathrm{~cm}^{-1}$ is obtained.

\subsection{Hopfield-Birge Systems (1810-950 ̊)}

a. $\mathrm{B}^{1} \Sigma^{+}, \mathrm{C}^{1} \Sigma^{+}, \mathrm{E}^{1} \Pi, \mathrm{F}\left({ }^{1} \Sigma^{+}\right),{ }^{6} \mathrm{G}\left({ }^{1} \Pi\right)$, and $b^{3} \Sigma^{+}-\mathrm{X}^{1} \Sigma^{+}$.

In 1927, there appeared preliminary reports by Hopfield [103] and Hopfield and Birge [104] concerning the observation of seven band systems of $\mathrm{CO}$ both in emission and absorption. These transitions, all going to the ground state, included four whose upper states had not previously been recorded: $a^{\prime 3} \Sigma^{+}, \mathrm{E}^{1} \Pi, \mathrm{F}\left({ }^{1} \Sigma^{+}\right)$, and $\mathrm{G}\left({ }^{1} \Pi\right)$. Strongest absorption was to the highest lying levels ( $G$, F, E, C). Full details of this work have never been published. Though several of the bands have since been observed elsewhere, only the $a^{\prime 3} \Sigma^{+}-\mathrm{X}^{1} \Sigma^{+}$system has been studied in detail [101] and will be discussed in section 3.4b. The other six transitions will be discussed together since the information about them is incomplete. Recently, however, high dispersion measurements have been made of the $\mathrm{C}-\mathrm{X}, 0-0$, and $1-0$ bands, with $v^{\prime}=1$ observed for the first time [276].

The observed band heads of all six systems are given in table 9 . The rotational constants for

\footnotetext{
O It is not certain whether the $F$ state is singlet or triplet. (See sec. $5.2 f$ and table 1 , footnote $F\left(1 \Sigma^{+}\right)$.)
}

the $\mathrm{B}, \mathrm{C}, \mathrm{E}$, and $b$ states are given, respectively in tables $38,39,40$, and 43 .

Read [209] observed several emission bands belonging to the $\mathrm{B}-\mathrm{X}, \mathrm{C}-\mathrm{X}$, and $\mathrm{E}-\mathrm{X}$ transitions. Tanaka et al. [255] have seen some of these in absorption. Since the $\mathrm{B}_{e}$ values of the three excited states and the ground state are roughly the same, each band had the appearance of a doublet with a clear center under low resolution. Identification of the $\mathrm{B}-\mathrm{X}, 2-0$ band [255] is rather uncertain.

Recently, Tilford et al. [257] have identified the $\mathrm{E}$ state as ${ }^{1} \Pi$ from analysis of the violetdegraded $0-0, \mathrm{E}-\mathrm{X}$ band observed in absorption under high dispersion. This identification confirmed the theoretical prediction by LefebvreBrion et al. [147]. A-doubling is about 0.2-0.3 $\mathrm{cm}^{-1}$ for $\mathrm{J} \sim 20$. (The $\mathrm{J}^{\prime}=31$ level is perturbed.) The isotopic $\left(\mathrm{C}^{13} \mathrm{O}^{16}\right) 0-0$ band was also analyzed. (Table 40.)

\section{b. $a^{\prime 3} \Sigma^{+}-\mathrm{X}^{1} \Sigma^{+}(1810-1280 \AA) \mathrm{R}$}

Of all the band systems of $\mathrm{CO}$ which were originally observed by Hopfield and Birge [103, 104], only the dipole forbidden $a^{\prime 3} \Sigma^{+}-X^{1} \Sigma^{+}$ system has been extensively studied (by Herzberg. and Hugo [101]). A long progression of bands has been observed up to $v^{\prime}=23$, because of the large difference in internuclear separation between the two states involved. The upper state of this system is responsible for many perturbations of the $A^{1} \Pi, b^{3} \Sigma^{+}$, and $a^{3} \Pi$ states.

The band heads and origins of the $a^{\prime}-\mathrm{X}$ system are given in table 10 . The rotational constants for the $\mathrm{X}$ and $a^{\prime}$ states are given in tables 36 and 41 , respectively.

Five bands of this system were first reported in absorption by Hopfield [103] with the one at $1696.9 \AA$ designated as the $0-0$ [104]. On the basis of an additional band found by Birge at $1731.2 \AA$, Estey [60] raised the vibrational quantum numbering in the $a^{\prime}$ state by one unit. The final assignment was deduced by Beer [16] from rotational perturbations in the $b^{3} \Sigma^{+}-a^{3} \Pi$ Third Positive bands caused by the $a^{13} \Sigma^{+}$state. This raised the quantum numbering of Hopfield and Birge by three units.

Recently, only two papers have dealt with this system [255, 101]. Tanaka, Jursa, and LeBlanc [255] have seen a long progression of these singleheaded red-degraded bands under low resolution. Because a short absorption cell was used, the $0-0$, 
1-0, and 2-0 bands were not observed. Herzberg and Hugo [101] observed this progression in absorption under high resolution in the region 1810-1280 $\AA$. These forbidden bands were photographed in fourth and filth orders of a $3-\mathrm{m}$ focal length spectrograph, with reciprocal dispersions of 0.63 and $0.50 \AA / \mathrm{mm}$, respectively.

In most of the bands [101] all four predicted branches, i.e., ${ }^{\mathrm{P}} \mathrm{P},{ }^{\mathrm{P}} \mathrm{Q},{ }^{\mathrm{R}} \mathrm{Q}$, and ${ }^{\mathrm{R}} \mathrm{R}$, are observed and give the appearance of a doublet structure. The order of the sublevels of the $a^{\prime 3} \Sigma^{+}$state is given as $\mathrm{F}_{3}>\mathrm{F}_{1}>>\mathrm{F}_{2}$.

The triplet splitting for the $a^{\prime}$ state is approximately constant for $N>5$. [ $N$ was formerly called K.] A discussion of splittings for $\mathrm{N}<5$ determined from other bands has been given by Gerö and Lörinczi [80]. The sum of splittings $[2 \lambda+\gamma]$ in corresponding members of the P and $R$ branches is approximately constant, with small change of $\lambda$ with $v$.

The rotational analysis results in the following formulas for the $a^{\prime 3} \Sigma^{+}$state.

$$
\begin{aligned}
\sigma_{0}= & 55822.92+1230.651\left(v^{\prime}+\frac{1}{2}\right)-11.013\left(v^{\prime}+\frac{1}{2}\right)^{2} \\
& +0.07378\left(v^{\prime}+\frac{1}{2}\right)^{3}-0.00115\left(v^{\prime}+\frac{1}{2}\right)^{4}-1081.59
\end{aligned}
$$

[Ref. 101 used 1081.74 for the ground state zeropoint energy.]

$$
\begin{aligned}
\mathrm{B}_{v}=1.3453- & 0.01872\left(v+\frac{1}{2}\right) \\
+ & 0.000205\left(v+\frac{1}{2}\right)^{2}-0.0000051\left(v+\frac{1}{2}\right)^{3} .
\end{aligned}
$$

$D^{\prime}$ was found to be about $6.5 \times 10^{-6}$ and varied little from $v^{\prime}=2$ to 23 .

Only a perturbation in $v^{\prime}=4$ at approximately $\mathrm{J}=20$ was observed. The perturbing level is $a^{3} \Pi, v=7$, which has not been observed directly. Other perturbations which occur at high $J$ (due to $A^{1} \Pi, a^{3} \Pi$, and $b^{3} \Sigma^{+}$) were not observed.

\section{5. $a^{3} \Pi-\mathrm{X}^{1} \Sigma^{+}$Cameron System (5800-1770 ̊) $\mathbf{R}$}

The $a^{3} \Pi-\mathrm{X}^{1} \Sigma^{+}$transition consists of quintupleheaded bands, degraded to the red, covering the spectral region $5800-1770 \AA$. This forbidden intercombination system is overlapped by the $\mathrm{B}^{2} \Sigma^{+}-\mathrm{X}^{2} \Sigma^{+}$First Negative bands of $\mathrm{CO}^{+}$and the A-X system of $\mathrm{CO}$. Recent high dispersion measurements by Rao [201] have provided an accurate value for the vibrational term interval $\Delta \mathrm{G}\left(\frac{1}{2}\right)$ of the $a^{3} \Pi$ state, but there is still lacking an accurate value of $\omega_{e} x_{\ell}$. The observed band heads of the $a^{3} \Pi-\mathrm{X}^{1} \Sigma^{+}$transition are listed in table 11 , the origins in table 12 . Rotational constants for the $a^{3} \Pi$ state are given in table 42 .

The band system bearing his name was first observed by Cameron [39] in an uncondensed discharge in neon with a trace of $\mathrm{CO}$; the following year it was seen in absorption by Hopfield and Birge [104]. Johnson [119] first identified these bands as due to transitions from the lower states of the 'Third Positive system to the ground state of $\mathrm{CO}$, and assigned vibrational quantum numbers to the bands. Knauss and Cotton [131] also observed these bands at low pressure in an electrodeless discharge in $\mathrm{CO}$.

Schmid and Gerö [232] observed the 4-8 band in a Geissler discharge in neon. Because of the coincidence of one head of this band and the intense red-degraded band reported by Kaplan [126], Schmid and Gerö believed that the level reported by Kaplan at $38820 \mathrm{~cm}^{-1}$ abore $\mathrm{X}$ was not real. (See footnote (K) of table 1.)

Herman [94] reported selective excitation of the $a-\mathrm{X}$ system between $5000-2000 \AA$ in a feeble discharge through $\mathrm{CO}$ at low temperature. Details are not given except for mention of the $R_{2}$ (or $\mathrm{Q}_{3}$ ) heads of the 2-4 and 0-1 bands, at $2299 \AA$ and $2159 \AA$, respectively. Comparison was made between the $R_{1}$ and $Q_{2}$ heads calculated from vibrational constants given by Johnson [119] and the faint low dispersion measurements of the night sky by a number of authors. The apparent agreement for a number of bands suggested to Herman the possible presence of the $a-\mathrm{X}$ system in the night sky. No other mention has been made of such correlation, and this agreement may be fortuitous.

Recently, Tanaka, Jursa, and LeBlanc [255] observed the $v^{\prime \prime}=0$ progression in absorption under low resolution. Unlike other intercombination bands observed by them, this progression did not derelop to high $v^{\prime}$ because of the Franck-Condon principle. [Note that ref. 255 lists vacuum wavelengths.]

The first high dispersion measurements of this system in absolption were those of Gerö, Herzberg, and Schmid [79] who give the fine structure analysis of the $0-0$ and $1-0$ bands. This was the first example of analysis of the fine structure of $n$ ${ }^{3} \Pi-{ }^{1} \Sigma$ transition. At least for large of the $a^{3} I I$ state is closo to Hund's caso (b). 'Thus the quantum number $N$ (formerly $\mathrm{K}$ ) has meaning for the rotational levels of both upper and lower 
states in this transition. Two equivalent notations of the nine branches are given below:

$$
\begin{array}{ccc}
\mathrm{P}_{1} \mathrm{Q}_{1} \mathrm{R}_{\mathrm{i}} & \mathrm{P}_{2} \mathrm{Q}_{2} \mathrm{R}_{2} & \mathrm{P}_{3} \mathrm{Q}_{3} \mathrm{R}_{3} \\
{ }^{\mathrm{o} P}{ }^{\mathrm{P}}{ }^{\mathrm{Q} R} & { }^{\mathrm{P} P}{ }^{\mathrm{Q} Q}{ }^{\mathrm{R}} \mathrm{R} & { }^{\mathrm{Q}} \mathrm{P}^{\mathrm{R}} \mathrm{Q}^{\mathrm{s}} \mathrm{R} .
\end{array}
$$

The combination differences which give $\Delta_{1} \mathrm{~F}_{1}$ for the ${ }^{3} \Pi_{0}$ component of the $a$ state do not coincide because of the very strong $\Lambda$-doubling of this component.

Gerö [76] used a Geissler discharge in neon with a trace of $\mathrm{CO}$ to excite (in emission) the $a-\mathrm{X}$ bands and, weakly, the $\mathrm{A}-\mathrm{X}$ system. His close examination of the work by Gerö, Herzberg, and Schmid [79] revealed the use of two unreliable standard iron lines by Schmid and Gerö, which accounted for a discrepancy in their wavelengths. Gerö tabulated the rotational lines for the $0-0$, $0-1,0-2,0-3,1-3,1-4$, and $2-5$ bands.

The most precise measurements of the $a-\mathrm{X}$ system have recently been made by Rao [201] who has given the rotational structure of the $0-0$, $0-1,0-2,0-3,1-3$, and $1-4$ bands seen in a Geissler discharge. A 21-ft focal length grating spectrograph was used in fourth and fifth order, with an iron arc providing a comparison spectrum. Errors in the combination differences for the $0-1$ band by Gerö were clarified. Relative accuracy of the line measurements [201] is estimated to be \pm 0.003 $\AA$. (A reproduction of the $0-1$ band is given in this reference.)

Rotational constants for the ground vibrational levels are close to those obtained from infrared measurements. Derived values for vibrational quanta for the ground state are the best available from electronic spectra of $\mathrm{CO}$, but are inferior to those determined from infrared measurements. A value of $1714.61 \mathrm{~cm}^{-1}$ is obtained for the vibrational quantum $\Delta \mathrm{G}\left(\frac{1}{2}\right)$ for the a state, confirming the value obtained previously [79].

\section{6. $b^{3} \Sigma^{+}-a^{3} \Pi$ Third Positive System (3820-2660 ^) V}

The Third Positive system of carbon, ${ }^{7}$ is composed of strong quintet-headed red-degraded bands whose structure is characterized by in-

\footnotetext{
7 Deslandres called the Third Positive group all [CO] bands in the UV region not belonging to the Fourth Positive group or Second Positive group ( $\AA$ ngstrom system). Some authors refer to the $v^{\prime}=0$ progression as Third Positive and call the $v^{\prime}=1$ progression " $5 \mathrm{~B}$ ". The two together should be treated as a single system.
}

tensity fluctuations due to perturbations of the upper state. Since the upper state is also predissociated, only the $v^{\prime}=0$ and 1 progressions are known, the latter having been dubbed by Asundi [4] as $5 \mathrm{~B}$, and at that time thought to be a new system. This is understandable because violent perturbations of the $b^{3} \Sigma^{+}$state drastically alter the relative appearance of the two progressions. The original measurements by Deslandres $[127 \mathrm{a}, 23]$ in 1888 , of emission from discharge tubes containing carbon and oxygen, included some bands down to $2295 \AA$ which turned out to be part of the $3 \AA$ system $\left(c^{3} \Sigma^{+}-a^{3} \Pi\right)$. The confirmed bands of the $b-a$ system extend from 3830-2660 $\AA$. These vibrational heads are given in table 13. The rotational constants for the $b^{3} \Sigma^{+}$and $a^{3} \Pi$ states are given in tables 43 and 42 , respectively.

The first assignment of vibrational quantum numbers of the $b^{3} \Sigma^{+}-a^{3} \Pi$ system was made by Johnson [119] who identified the lower state with the upper state of the Cameron bands. Previously Wolter [277], using high resolution, had observed five bands of the $v^{\prime}=0$ progression. He mentioned the repeated rise and fall of intensity of the band lines and, in addition, reported the appearance of weak diffuse lines between the main lines. Later this system was observed by Duffendack and Fox $[57,58]$ who concluded that the group of bands labeled by Johnson [119] as the $v^{\prime}=4$ progression constituted the $v^{\prime}=0$ progression of a new system called $3 \mathrm{~A}$.

Asundi [4] observed the $b-a$ and $3 A$ bands in an uncondensed discharge using a $21-\mathrm{ft}$ focal length grating spectrograph. Outside the first (longest wavelength) head of these violet-degraded bands were faint lines which decreased in spacing toward higher wavelengths, formed a diffuse head, and continued further with a gradual increase in spacing. Asundi attributed the $b-a$ system to a ${ }^{5} \Sigma-{ }^{5} \Pi$ transition. Birge [23] disagreed and considered the transition as ${ }^{3} \Sigma-{ }^{3} \Pi$, an assignment later firmly established by Dieke and Mauchly [54].

An extensive discussion of the spin splitting and $\Lambda$-doubling in these bands has been given by Dieke and Mauchly [54], who observed the 0-0 to $0-4$ bands using dispersion of $<1 \AA / \mathrm{mm}$. Budó [32] has used these data to obtain rotational constants for the $a^{3} \Pi$ state. Dieke and Mauchly's analysis established the transition as ${ }^{3} \Sigma^{+}-{ }^{3} \Pi$, with triplet (spin) separation of the $b^{3} \Sigma$ state unnotice- 
able for $J<20$. The character of the $\Lambda$-doubling and the number of missing lines near the origin identified the lower state as regular ${ }^{3} \Pi$, intermediate in coupling between case (a) and (b). From a study of the Zeeman effect on this band system Schmid [236] showed that, beginning at about $\mathrm{N}=22-23$, the $a^{3} \Pi$ state belonged to coupling case (b). The ${ }^{3} \Sigma$ terms have separation $<0.2 \mathrm{~cm}^{-1}$ for $\mathrm{N}$ up to about 20 . The terms in order of decreasing energy are anomalous: $\mathrm{F}_{1}>\mathrm{F}_{2}>\mathrm{F}_{3}$.

The $b-a$ bands represent a transition from case (a) to case (b); i.e., the ${ }^{3} \Pi$ state already departs from case (a) coupling for small N. Fifteen branches are allowed by the selection rules, of which fourteen were observed as well as the trace of a fifteenth. Nine strong bands indicate coupling close to case (b). The five head-forming branches are $\mathrm{O}_{3}, \mathrm{O}_{2}, \mathrm{P}_{3}, \mathrm{P}_{2}$, and $\mathrm{P}_{1}$.

Many large perturbations, mostly far from the heads, cause the characteristic intensity fluctuations first mentioned by Wolter [277], and cause clustering of lines toward the tails. At high pressure the tails show characteristic triplets especially in the $0-1$ band at $2977 \AA$. [See fig. 2 of ref. 54b.] In front of the heads (at shorter wavelength) are faint lines forming very weak series which do not form heads. Dieke and Mauchly showed that these are due to transitions with $\Delta N=3$. It is not certain whether these were the lines found by Asundi [4].

Schmid and Gerö [226] have reported clustering of Q-branch lines in the $v^{\prime}=1$ progression caused by perturbations of the $b^{3} \Sigma^{+}$state. The positions of these perturbations converge to a limit which seems to coincide with the predissociation limit common to the $v^{\prime}=0$ progression and to the $\mathrm{B}$ state. Gerö's [75] report on the fine structure of the 1-0 band has confirmed the predissociation of the upper state between $\mathrm{N}=42$ and 43 .

Rotational analysis of the $0-4,0-5,1-4$, and 1-5 bands has been given by Beer [16]. Perturbation of $v^{\prime \prime}=4$ was observed and assigned to $a^{\prime 3} \Sigma^{+}$.

The molecular constants for the $b$ state are somewhat uncertain. Schmid and Gerö's values [226] are quoted by Hel\%berg [99]; i.e., $\mathrm{B}_{e}=2.075$, $\alpha_{e}=0.033, \mathrm{~B}_{1}=2.025 \mathrm{~cm}^{-1}$. Stepanov [246] has criticized the way these values were determined and his values are listed in table 1, i.e., $B_{e}=1.986$, $\alpha_{e}=0.042 \mathrm{~cm}^{-1}$. For $\Delta \mathrm{G}\left(\frac{1}{2}\right)$ from measurement of $b-a$ bands Schmid and Gerö [226] obtrined 2109 $\mathrm{cm}^{-1}$. Jevons [117] had previously listed 2214 $\mathrm{cm}^{-1}$ from the data of Asundi [4]. Gerö's [75] estimate of $2198 \mathrm{~cm}^{-1}$ is the value listed in Herzberg. This value was obtained by extrapolation to $\mathrm{N}=0$ of the difference between the $\mathrm{Q}$ lines of the 1-0 and 0-0 bands. Stepanov [246] criticized this value and used a different method to obtain $2188 \mathrm{~cm}^{-1}$ which is the value quoted in table 1. These constants should be accepted as only tentative and renewed attempt should be made to determine them more precisely.

\section{7. $c^{3} \Sigma^{+}-a^{3} \Pi$ II System $(2710-2300$ A) V}

Duffendack and Fox [57, 58] measured the excitation potentials of several band systems of $\mathrm{CO}$, including five bands of a new system appearing at about $11.1 \mathrm{eV}$. These bands had the same final state as the Third Positive system, $\left[b^{3} \Sigma^{+}-a^{3} \Pi\right]$, but originated from a state about $1.02 \mathrm{eV}$ above the $b$ state. The new system was labeled $3 \mathrm{~A}$, and bands were identified as the $\left(0-v^{\prime \prime}\right)$ progression for $v^{\prime \prime}=0$ to 4 . The violet-shaded bands were multiple headed.

Faint, five-headed bands of this system were produced by Asundi [4] in an uncondensed discharge in $\mathrm{CO}$. The first and fifth heads are strongest; hence, under low dispersion the bands appear double-headed. The electronic multiplet separation of the measured extreme heads is largest for the 0-0 band, and, as with the Third Positive bands, decreases for higher vibrational states.

An equation for the heads is given by Asundi [4]

$$
\sigma=\frac{43542.5}{\bar{\square}}-\left(1726.5 v^{\prime \prime}-14.4 v^{\prime \prime 2}\right) .
$$

Schmid and Gerö [230] excited intense $3 \mathrm{~A}$ bands in a discharge through neon in a Geissler tube with carbon electrodes and gave a preliminary report on the $0-1,0-2$, and $0-3$ bands. 'They detected 12 branches in each band, the remaining branches being weak and overlapped. Six branches are head forming: $\mathrm{O}_{3}, \mathrm{O}_{2}, \mathrm{P}_{3}, \mathrm{P}_{2}, \mathrm{P}_{1}$, and $\mathrm{Q}_{1}$ in order of diminishing wavelengths. The nature of the branches shows the upper state to be ${ }^{3} \Sigma$, with negligible multiplet splitting.

Gerö [77] reported the detailed rotational analysis of the $0-1,0-2$, and $0-3$ bands observed at a dispersion of $1.3 \AA / m m$. A predissociation in this system is observed at about $11 . \dot{\mathrm{eV}}$. 'The 
combination differences for the $a^{3} \Pi$ state agree well with those of the $b-a$ system which confirms the order of the bands and band lines and the $\Sigma^{+}$character of the $c$ state. $\quad A$ value of $\mathrm{B}_{0}=1.9563$ $\mathrm{cm}^{-1}$ is derived.

The last observed lines of the $R_{3}$ branch of the 0-1 3A band lies in the region of a spurious predissociation in the $\mathrm{P}$ branch of the red-degraded 9-18 Fourth Positive band. The rotational analysis of the $3 \mathrm{~A}$ bands shows that the apparent increase of intensity of some of the Fourth Positive band lines [74] in this region are caused by superposition of certain $3 \mathrm{~A}$ lines.

The band heads of the $3 \mathrm{~A}$ system are given in table 14 , and the rotational constant $\mathrm{B}_{0}=1.9563$ is included in tables 1 and 44 .

\section{8. $a^{\prime 3} \Sigma^{+}-a^{3} \Pi$ Asundi System (8590-3900 ̊) R}

The multiheaded bands of the $a^{\prime}-a$ system have been studied mostly under low dispersion, with only a few rotational constants determined for the $a^{\prime}$ state from their analysis. More accurate values for these constants are available from analysis of another system [101]. Two bands which were believed to originate from levels with $v \geq 30$ in the $a^{\prime}$ state [78] most probably belong to a new ${ }^{3} \Sigma^{+}$state whose term value is uncertain. The observed vibrational heads of the $a^{3} \Sigma^{+}-a^{3} \Pi$ system are given in table 15 . Rotational constants for the $a^{\prime}$ and $a$ states are given in tables 41 and 42 , respectively.

McLennan, Smith, and Peters [158], using low dispersion, observed a large number of reddegraded bands produced in a long, high-voltage discharge tube filled with $\mathrm{CO}$. (A number of these bands which have not been identified are listed in table 35c.) Shortly thereafter Asundi [4] discovered 16 red-degraded bands of the system bearing his name which were overlapped by the $d^{3} \Delta_{i}-a^{3} \Pi$ Triplet bands below $5700 \AA$. The $a^{\prime}-a$ bands appear double headed under low dispersion, but have five heads when seen under higher dispersion.

By assuming the band at $8592 \AA$ was the $1-0$, Asundi assigned vibrational quantum numbers. A new term of the $a^{\prime}-\mathrm{X}$ system found by Estey (at $57763 \mathrm{~cm}^{-1}$ ) [60] required the $v^{\prime}$ assignments to be raised by unity. The correct $v^{\prime}$ numbering was finally established by Beer [16] who indicated that a perturbation of the $a^{3} \Pi, v=4$ term is caused by $a^{\prime 3} \Sigma^{+}, v=0$, which required Asundi's original $v^{\prime}$ values to be raised by three. [See ref. 80.]
Knowledge of the $a^{\prime}-a$ system was considerably extended by the observations of Garg [68]. Numerous impurity $\mathrm{CO}$ bands were observed during the preparation of a discharge tube for the study of emission from $\mathrm{SnBr}_{4}$ vapor. The band positions are assumed accurate to $\pm 4 \mathrm{~cm}^{-1}$. Garg's formula, fitted to both his and Asundi's measurements, is given below with $v^{\prime}$ numbering of Gerö and Lörinczi [80].

$$
\begin{aligned}
\sigma_{\mathrm{H}}=7213.8+1218.1 & \left(v^{\prime}+\frac{1}{2}\right)-9.5\left(v^{\prime}+\frac{1}{2}\right)^{2} \\
& -1740.9\left(v^{\prime \prime}+\frac{1}{2}\right)+14.4\left(v^{\prime \prime}+\frac{1}{2}\right)^{2} .
\end{aligned}
$$

A few bands which cannot be fitted in this system are listed separately, and assumed members of an unknown system. (See table 35c.) Garg's table of band heads shows increasing deviation of observed-calculated differences for $v^{\prime} \geq 16$. It is not presently known whether such deviations arise from perturbations, or are due to incorrect identification of particular subheads used to calculate these deviations.

Schmid and Gerö [233], in a note, reported several red-degraded bands near the heads of the violet degraded $b^{3} \Sigma^{+}-a^{3} \Pi$ Third Positive system. An especially strong band is observed at $2670 \AA$ in a Geissler tube discharge in neon with carbon electrodes, in the presence of very little oxygen. In another brief communication Schmid and Gerö [231] ascribed the bands as due to a ${ }^{3} \Sigma-a^{3} \Pi$ transition. The upper state, provisionally designated as $f^{3} \Sigma$, was suspected as including high vibrational levels of the $a^{\prime 3} \Sigma$ state. This was supposedly confirmed by Gerö [78], but Herzberg [99] believed the bands belonged to another state and confirmed this indirectly in later work [101].

Gerö and Lörinczi [80] hare given the rotational analysis of the $8-0,9-0,10^{\circ}-1$, and $11-1$ bands which were observed in emission. The $8-0$ band is weaker than the rest and its branches are not given. The high dispersion measurements are relatively free of orerlap by other systems, and allowed measurement of lines to a few hundredths $\mathrm{cm}^{-1}$. Rotational constants for $a^{\prime 3} \Sigma^{+}, v=8$ to 11 are obtained. A long extrapolation of these [which is usually dangerous] gives the molecular constants $\mathrm{B}_{e}=1.331 \mathrm{~cm}^{-1}$ and $\alpha_{e}=0.016 \mathrm{~cm}^{-1}$ which are the values listed by Herzberg [99]. The rotational constants for the upper levels are smaller by only about 1.5 percent than those given previously from perturbation data [234a]. From more recent work on the $a^{\prime 3} \Sigma^{+}-\mathrm{X}^{1} \Sigma^{+}$ system, Herzberg and Hugo [101] obtained im- 
proved constants $\mathrm{B}_{e}=1.3453, \alpha_{e}=0.01872$, and $\gamma_{e}=0.000205 \mathrm{~cm}^{-1}$.

The spin splitting of the ${ }^{3} \Sigma$ state is rather large. For the 10-1 band, the splitting is constant above $\mathrm{N}=5$. The splitting (in $\mathrm{cm}^{-1}$ ) is almost the same for each of the bands as is seen below:

\begin{tabular}{r|c|r}
\hline \hline & $F_{3}-F_{2}$ & $F_{1}-F_{2}$ \\
\hline & & \\
\cline { 2 - 2 } $10-0$ & 1.40 & 0.97 \\
$11-1$ & 1.37 & .98 \\
& 1.40 & 1.01 \\
\hline
\end{tabular}

(See ref. 80 for a correction to table 2 of ref. 78).

\section{9. $d^{3} \Delta_{i}-a^{3} \Pi$ Triplet System (7500-3770 ̊) $\mathbb{R}$}

The $d^{3} \Delta_{2}-a^{3} \Pi$ Triplet bands of $\mathrm{CO}$ are a fairly extensive system of moderate intensity spanning the visible region of the spectrum. Merton and Johnson [160], in studying the effect which the presence of helium had on band spectra of carbon, first isolated two progressions of this system. These red-degraded bands were later assigned to CO when Birge [21] identified the lower state as $a^{3} \Pi$ and gave a vibrational analysis. Extension of the system to longer wavelengths by Asundi [7] required an increase in $v^{\prime}$ assignments by two units. Carroll [40] has reported that the numbering for the $d$ state vibrational levels should be increased by one more unit. Only recently the upper state was identified [40] as ${ }^{3} \Delta_{i}$, following a prediction of the $\Delta$ character by Mulliken [171]. Kovács [137] has explained the inverted character of this state which is not expected in first order from a $\pi^{3} \pi$ configuration. Band heads are given in table 16; origins in table 17 . Rotational constants for the $d^{3} \Delta_{i}$ state are given in table 45 .

Asundi [7], from study of three bands at longer wavelength, concluded that the band at 15540 $\mathrm{cm}^{-1}$ was not $0-0$, and that the $v^{\prime}$ assignments as listed by Gerö and Szabó [82] should be raised by two. The bands were found to have five lueads. He was not certain whether his band at $13303 \mathrm{~cm}^{-1}$ was definitely $0-0$, and speculated that the system origin might be at longer wavelength than his range of observation.

Herman and Herman [92, 96] observed emission of $d^{3} \Delta_{i}-a^{3} \Pi$ Triplet bands in a low current discharge in neon with traces of $\mathrm{CO}$ at liquid nitrogen temperatures. Under these conditions bands with high $v^{\prime}$ predominate. Bands with $v^{\prime}=6$ [add 3 to the $v^{\prime}$ assignments of Herman and Herman] are relatively weak compared to intense bands for $v^{\prime}=5$ and 7 . The bands with $v^{\prime}=12$ are relatively weak and those with $v^{\prime}=13$ are enhanced compared with those for $v^{\prime}=14$. A new system of triple-headed bands was also observed and is discussed further in section 3.14.

Recently Sato [219] used a $33.3 \mathrm{MHz}$ discharge to produce more than 40 rather intense bands of the $d-a$ system. His low dispersion measurements included almost all bands observed previously as well as the $12-0,5-1,6-1,6-2,7-2$, $17-2,18-2,19-3,20-3,17-4,21-4$, and $22-4$. His $v^{\prime}$ values should be raised by three units. Uncertainty in the shorter wavelengths is about $0.5 \AA$; uncertainty in the longer wavelengths is about $1 \AA$.

For a number of bands, Sato's measurements differ considerably from those of several other authors. This raises questions about quantum assignments for other bands observed by Sato. This system should be reexamined to clarify the band identification.

Gerö and Szabó [82] have given the rotational analysis for the $3-0,4-0,6-0,7-0$, and $9-1$ bands. (Their original $v^{\prime}$ assignments are too low by three units.) The fine structure was interpreted as showing the transition to be $d^{3} \Pi_{i} \rightarrow a^{3} \Pi_{r}$. The upper state was later correctly identified as ${ }^{3} \Delta_{i}$ by Carroll [40]. Gerö and Szabó observed these bands in a Geissler discharge in neon with traces of oxygen. The bands also appeared under favorable conditions without the presence of a noble gas. In the latter case there was orerlap with the Angstrom and Asundi bands, $\mathrm{B}^{1} \Sigma^{+}-\mathrm{A}^{1} \Pi$ and $a^{\prime 3} \Sigma^{+}-a^{3} \Pi$, respectively.

Twenty-seven branches were observed, doubled because of $\Lambda$-doubling. It was established that $\Lambda$-doubling of the band lines agreed with that of the $a^{3} \Pi$ state (from the $b^{3} \Sigma^{+}-a^{3} \Pi$ Third Positive bands) and that the $\Lambda$-doubling of the $d$ term was immeasurably small. Rotational constants, collpling constants $A$, and $Y=\frac{A}{B}$ for the $d$ state were calculated from combination differences and the $\mathrm{F}_{1}-\mathrm{F}_{3}$ differences based on Budó's formulas [32b] for a transition between two triplet $\Pi$ states in intermediate coupling between cases (a) and (b). Corrected values are given by Carroll [4(0]. 'The origins of four bands were calculated by Gerö and Szabo [52] using an arerage of nine (Q-branches whose series were extrapolated to $J=0$. Based on these zero positions the vibrational tem dilfor- 
ences were calculated for the $d$ term. A formula fitted to the correctly numbered $v^{\prime \prime}=0$ progression is given here as $\sigma_{0}\left(v^{\prime}-0\right)=11598.50+1152.58$ $\left(v^{\prime}+\frac{1}{2}\right)-7.2812 \quad\left(v^{\prime}+\frac{1}{2}\right)^{2}-0.1125 \quad\left(v^{\prime}+\frac{1}{2}\right)^{3}$ which gives $\sigma_{0}(0-0)$ at $12,172.96 \mathrm{~cm}^{-1}$.

Carroll [40] has given the rotational analysis of the 3-0 band based on high resolution measurements and has shown the upper state to be ${ }^{3} \Delta_{i}$ in agreement with electron-configuration theory and not ${ }^{3} \Pi_{i}$ as indicated by Gerö and Szabó [82]. Mulliken [171], on the basis of LCAO-MO calculations for the lower excited states of $\mathrm{CO}$, had predicted a ${ }^{3} \Delta$ state at about the observed energy. Carroll's work was initiated to clarify the nature of the upper state, and is the first reported example of a ${ }^{3} \Delta \rightarrow{ }^{3} \Pi$ transition with nonnegligible (multiplet) splitting. The source used was a discharge through flowing helium with a trace of CO. Of the several bands observed, the one at $6401 \AA$ was analyzed because it extended to high $J$ and was relatively free from overlapping by other structure. All 27 branches were observed, although sometimes the $\Lambda$-doublets were not resolved for lower J. There are nine subbands, each composed of three doublet branches, although the branches going to ${ }^{3} \Pi_{2}$ of the lower state are only resolved at higher $J$. The ${ }^{3} \Delta_{i}$ state is case (a) at low $J$ and changes to case (b) at higher $J$ (See fig. 1 of ref. 40.) The a state also is case (a) at lower J.

Carroll's measurements agree well with the previous ones of Gerö and Szabó for the $3-0$ band, with several differences in classification of lines. In addition, some of Gerö's assignments at low J seem to be spurious. Detailed evidence is given for the assignment of the upper state as ${ }^{3} \Delta_{i}$, including confirming evidence from the intensity distribution in the fine structure. At that time there had been no theoretical calculation of branch intensities for a ${ }^{3} \Delta-{ }^{3} \Pi$ transition, but such formulae have since been obtained by Kovács [136], for various coupling conditions of the states involved.

The energy formulas of Budó [32b], although good to a first approximation, do not give the rotational term values of the ${ }^{3} \Delta_{i}$ state with sufficient accuracy [40]. This deviation between the observed and calculated energies, which affects most strongly the middle multiplet component, is explained by Kovács [137] as arising from the joint effect of two factors normally neglected: (1) spin- spin interaction, and (2) spin-orbit interaction by mixing with a nearby state, probably ${ }^{1} \Delta$. No ${ }^{1} \Delta$ state has been observed directly, though a possible perturbation of $A^{1} \Pi, v=0$ by such state has been mentioned by Rosenthal and Jenkins [216].

\subsection{0. $e^{3} \Sigma^{-}-\mathrm{X}^{1} \Sigma^{+}$Transition (1540-1240 $\left.\mathrm{A}\right) \mathrm{R}$}

A forbidden electronic transition, $e^{3} \Sigma^{-}-\mathrm{X}^{1} \Sigma^{+}$, has been observed by Herzberg and Hugo [101] in the vacuum UV region. They observed a long $v^{\prime \prime}=0$ progression of weak, red-degraded single headed bands in absorption by use of a 3-m focal length vacuum spectrograph, at dispersions of 0.63 and $0.50 \AA / \mathrm{mm}$. These bands have also been seen under low dispersion by Tanaka, Jursa, and LeBlanc [255]. The existence of the $e^{3} \Sigma^{-}$ state had previously been inferred from perturbations in the $\mathrm{B}-\mathrm{A}$ bands [101] and in the $\mathrm{A}-\mathrm{X}$ bands [234b]. Many $e-\mathrm{X}$ bands are orerlapped by bands of the $\mathrm{A}-\mathrm{X}, a^{\prime}-\mathrm{X}$, and $d-\mathrm{X}$ transitions [101, 255]. Table 18 lists the band heads and origins of the $e-\mathrm{X}$ system. Rotational constants for the $e^{3} \Sigma^{-}$ state are given in table 46 .

The observed progression began with a relatively strong band at $64816 \mathrm{~cm}^{-1}$ which was labeled $0-0$. Since then, two bands have been found at longer wavelength [100] which suggested raising the $v^{\prime}$ values reported earlier by two units. Fine structure analysis of these bands [238a] showed conclusively that the $v^{\prime}$ values of Herzberg and Hugo should be raised by unity.

Fine structure has been determined for the $5-0$, $6-0,7-0,9-0,11-0$, and $13-0$ bands, which resemble a ${ }^{1} \Pi-{ }^{1} \Sigma$ transition, but with the $\mathrm{Q}$ branch doubled. Electric dipole selection rules $(+\leftrightarrow-$, $\Delta \mathrm{J}=0, \pm 1$ ) indicate five branches are expected for $a^{3} \Sigma^{-}-{ }^{1} \Sigma^{+}$transition which is possible because of spin-orbit interaction: $\mathrm{Q},{ }^{\mathrm{Q}} \mathrm{P},{ }^{\mathrm{Q}} \mathrm{R},{ }^{\mathrm{s}} \mathrm{R}$, and ${ }^{\mathrm{O}} \mathrm{P}$. Recall that the selection rule prohibiting $\Sigma^{-}-\Sigma^{+}$ transition does not apply to intercombinations [220]. In the ${ }^{3} \Sigma$ state, $\mathrm{F}_{1}$ and $\mathrm{F}_{3}$ sublevels ( $\mathrm{J}=$ $\mathrm{N} \pm 1$ ) lie close together, with the $\mathrm{QP}$ and $\mathrm{QR}$ branches nearly coincident and separated from the $\mathrm{Q}$ branch. These conclusions are confirmed in the observation of the $8-0$ band, for in most other bands, the three Q-form branches are not resolved. Herzberg and Hugo [101] have made no quantitative intensity measurements, but have compared the intensity factors of the Q-form branches as given by Schlapp [220]. 
The formulas fitted to their data (with revised quantum numbering) are given below:

$$
\begin{array}{r}
\mathrm{B}_{v}{ }^{\prime}=1.2848-0.0181\left(v^{\prime}+\frac{1}{2}\right)+0.0001\left(v^{\prime}+\frac{1}{2}\right)^{2} \\
\sigma_{0}\left(v^{\prime}-0\right)=64236.32+1113.167\left(v^{\prime}+\frac{1}{2}\right)-9.596 \\
\left(v^{\prime}+\frac{1}{2}\right)^{2}+0.00587\left(v^{\prime}+\frac{1}{2}\right)^{3}-1081.59
\end{array}
$$

where the last number is the zero-point energy of the $\mathrm{X}$ state.

\subsection{1. $\mathrm{C}^{1} \Sigma^{+}-a^{\prime 3} \Sigma^{+}$Knauss System (3250-2930 A) V}

Knauss [130], in an abstract, reported four violet-degraded bands obtained in an electrodeless discharge through $\mathrm{CO}$. These were identified as belonging to $\mathrm{C}^{1} \Sigma^{+}-a^{\prime 3} \Sigma^{+}$(table 19). More recent data on the $a^{\prime}$ state indicates that the $v^{\prime \prime}$ values should be two units larger than those quoted by Knauss.

\subsection{Kaplan Bands (2750-2520 ̊) V}

A. In a brief note Kaplan [125] reported three bands forming a new system when a trace of $\mathrm{CO}$ was excited in a long atomic hydrogen tube. The bands resembled the $3 \mathrm{~A}$ system $\left(c^{3} \Sigma^{+} \rightarrow a^{3} \Pi\right)$, each having six heads and degraded to the violet. The transition is attributed to $\mathrm{K}-a^{3} \Pi$, the upper state being tentatively identified by Kaplan as a metastable quintet. These bands do not appear under ordinary conditions. (Schmid and Gerö [227] have speculated that these bands may be the $v^{\prime}=2$ progression of the $b-a$ Third Positive system.) Assuming $v^{\prime}=0$ yields $T_{0}=89889 \mathrm{~cm}^{-1}$ (above $\mathrm{X}$ ) for the upper level. The bands are listed in table 20 .

B. Kaplan [126], in an abstract, reported an intense single band at $2575 \AA$, similar in structure to the $A^{1} \Pi-X^{1} \Sigma^{+}$Fourth Positive bands, hence degraded to the red. This band was discovered in quenching mercury resonance radiation by $\mathrm{CO}$. Schmid and Gerö [232] indicated that this band coincided with a head of the $4-8, a^{3} \Pi-\mathrm{X}^{1} \Sigma$ Cameron band (which according to Cameron himself was the most intense band of the system) and disputed the reality of the $2575 \AA$ band as due to a new CO transition. Schmid and Gerö [234b] indicated that the disputed band is strongly orerlapped by the $0-0$ band of $A^{1} \Pi-\mathrm{X}^{1} \Sigma$ of $\mathrm{CS}$ in a variety of discharges and this level of $\mathrm{CO}$ would appear to be illusory, and should not be included among $\mathrm{CO}$ terms.

\subsection{3. $d^{3} \Delta_{t}-\mathrm{X}^{1} \Sigma^{+}$Transition $(1620-1230 \AA) \mathrm{R}$}

In a study of absorption spectra of $\mathrm{CO}$ in the vacuum ultraviolet region, Tanaka, Jursa, and LeBlanc [255] observed a weak progression of single headed bands extending to high $v^{\prime}$, assumed to correspond to the $R$ heads of the transition. At that time the upper state was thought to be ${ }^{3} \Pi_{i}$, and is now known to be ${ }^{3} \Delta_{i}$. The $v^{\prime}$ values given in reference [255] need to be raised by one unit. (See the discussion of $v^{\prime}$ numbering in the Triplet $d-a$ bands.) The band heads are listed in table 21.

\subsection{4. $e^{3} \Sigma^{-}-a^{3} \Pi$ Herman System $(5430-4270 \AA)$ $\mathrm{R}$}

In a study of the $d^{3} \Delta_{i}-a^{3} \Pi$ Triplet bands, Herman and Herman [96, 92] reported a new progression of emission bands similar in appearance [red-degraded], but having more closely spaced heads. 'These bands were attributed to a new system whose upper state was assumed to be $d$ (then thought to be ${ }^{3} \Pi$ ) and the lower state assumed to be a new triplet level lying near $a^{3} I$. Long ago Merton and Johnson [160] had observed some of these bands, but had not identified them. By using the absorption data of Herzberg and Hugo [101] and that of Tanaka, Jursa, and LeBlanc [255], Barrow [12] has shown that the Herman bands constitute the $\left(v^{\prime}-0\right)$ progression of the $e^{3} \Sigma^{-}-a^{3} \Pi_{r}$ iransition (table 22). The $v^{\prime}$ numbering of Barrow should be raised by unity [238a]. "It is anticipated that other" bands of the Herman system will probably be found at longer wavelengths" [12].

3.15. $\mathrm{E}_{0}{ }^{1} \Sigma^{+}-\mathrm{X}^{1} \Sigma^{+},{ }^{1} \Pi-\mathrm{X}^{1} \Sigma^{+}$, and Several Unidentified Transitions in the Vacuum UV Region (1180-930 ^)

In a discharge in $\mathrm{CO}$ at several mm pressure Tschulanowsky and Gassilewitsch [261] excited a number of weak bands in the region 1200-930 $\AA$. Included among the bands is the single band of the E-X Hopfield-Birge transition. Most of the bands are unclassified and are listed as such in in table 35d. Several bands belong to a new system labeled $E_{0}{ }^{1} \check{-X} X^{1}$ by 'Tschulanowsky [260] (see table 23). The $\left(0-r^{\prime \prime}\right)$ progresion of 
red-degraded bands has a system origin at 90866.2 $\mathrm{cm}^{-1}$. The relative error is estimated to be 0.01 to $0.03 \AA$ (or less than $3 \mathrm{~cm}^{-1}$ ). The constant $v^{\prime}$ value is uncertain and was assumed to be zero. A formula is obtained which fits the heads: $\sigma\left(0-v^{\prime \prime}\right)=90869.45-\left(2171.19 \quad v^{\prime \prime}-14.28 \quad v^{\prime 2}\right)$, giving constants which deviate considerably from those of the ground state. On this basis the reality of the $\mathrm{E}_{0}$ state is questioned.

For the most intense band at $1183.62 \AA$, a value of $\left(\mathrm{B}^{\prime}-\mathrm{B}^{\prime \prime}\right)=-0.6882 \mathrm{~cm}^{-1}$ was obtained. Both branches observed were of comparable intensity. By using $B^{\prime \prime}=1.8702 \mathrm{~cm}^{-1}$ one obtains $\mathrm{B}_{0}^{\prime}=1.182$ $\mathrm{cm}^{-1}$ (which is given in table 47 ), $r^{\prime} \sim 1.44 \AA$, and $\sigma_{0}(0-0)=90866.2 \mathrm{~cm}^{-1}=1100.52 \AA$. This suggests a very weak bond and a state with a flat potential curve. The $B_{0}^{\prime}$ is not considered accurate, but a rough confirmation of its magnitude was obtained from a partial analysis of two other bands. It is estimated that $\omega_{e}$ for the $\mathrm{E}_{0}$ state $<1000 \mathrm{~cm}^{-1}$ (comparable to the $b^{\prime 1} \Sigma_{u}^{+}$state of $\mathrm{N}_{2}$ ).

Tschulanovsky [260] has also reported a group of weak, overlapped red-degraded bands in the region of $1100-1000 \AA$ which form a new ${ }^{1} \Pi-X$ system. The measurements are not very reliable. The best resolved band is the $0-1$ at $1034.65 \AA$, which shows the $P$ and $R$ branches longer and more intense than the $Q$. A value of $\left(B^{\prime}-B^{\prime \prime}\right)=$ $-0.7659 \mathrm{~cm}^{-1}$ gives $\mathrm{B}^{\prime}=1.139$ (see table 48) using $B_{0}^{\prime \prime}=1.9052 \mathrm{~cm}^{-1}$. The calculated origin of the ${ }^{1} \Pi-\mathrm{X}^{1} \Sigma^{+}$system is at $98836 \mathrm{~cm}^{-1}$. The bands are given in table 24 .

\subsection{6. $f^{3} \Sigma^{+}-a^{3} \Pi$ Transition (2980-2670 $\AA$ ) $\mathbb{R}$}

Schmid and Gerö [233] have observed several red-degraded bands (table 25) near the heads of the 1-0 and $0-1$ violet-degraded $b-a$ Third Positive system. An especially strong band appeared at $2670 \AA$ in a Geissler tube discharge (between carbon electrodes) in neon, in the presence of very little oxygen. Schmid and Gerö [231] ascribed the bands to a ${ }^{3} \Sigma^{+}-a^{3} \Pi$ transition. The upper state, provisionally designated as $f^{3} \Sigma^{+}$, was suspected as including high vibrational levels of the $a^{3} \Sigma^{+}$ state. The supposed confirmation of this assignment by Gerö [78] was not fully accepted by Garg [68], Gaydon [69], and Herzberg [99]. The work of Herzberg and Hugo [101] on the $a^{\prime}-\mathrm{X}$ transition implies that the Schmid and Gerö bands do not belong to the $a^{\prime}-a$ system.

Gerö [78] has given the rotational analysis of the two $f-a$ bands which he labeled the $30-1$ and
34-0 bands of the $a^{\prime}-a$ transition. Stepanov [247] labeled these with $v^{\prime}$ increased by one unit. For the $a^{3} \Pi$ state in coupling case (a), 27 branches are allowed. Relatively large triplet splitting of the $f^{3} \Sigma^{+}$terms is indicated since most of these were observed. Spin splitting $\left(\mathrm{cm}^{-1}\right)$ is found to be roughly independent of $N$ :

\begin{tabular}{l|c|c}
\hline \hline & $2979.9 \AA$ & $2669.7 \AA$ \\
\hline$F_{1}-F_{2}$ & 0.80 & 1.05 \\
$F_{3}-F_{2}$ & 1.08 & 1.43 \\
\hline
\end{tabular}

(See p. 461 of ref. 80 for a correction to Gerö's paper.) Perturbations are indicated near $\mathrm{N}=0$ of the $f$ state, presumbably caused by $b^{3} \Sigma^{+}$. Rotational constants for the $f$ state are estimated using the unperturbed levels with higher $\mathrm{N}$ (table 49). The unperturbed term values of the upper state vibrational levels are estimated to lie at 83744 and $85969 \mathrm{~cm}^{-1}\left(36 \mathrm{~cm}^{-1}\right.$ has been added to Gerö's figures [78] to account for revised $a-X$ data). Stepanov [247[ obtained values close to those of Gerö which, when revised, would give 83755 and $85960 \mathrm{~cm}^{-1}$.

The small B values ( 0.83 and $0.75 \mathrm{~cm}^{-1}$ ) for the $f$ state terms imply either high vibrational quantum states or a weakly bound electronic state with large $r_{e}$. This rather peculiar structure should be re-examined experimentally.

\subsection{7. $\mathbb{I}^{1} \Sigma^{-}-\mathrm{X}^{1} \Sigma^{+}$Transition $(1520-1460$ A $) \mathrm{R}$}

Three red-degraded bands consisting of a single Q branch have been observed in absorption [100] under high dispersion and correspond to the forbidden $\mathrm{I}^{1} \Sigma^{-}-\mathrm{X}^{1} \Sigma^{+}$transition. Rough measurements of wavelength indicate a vibrational frequency near $1000 \mathrm{~cm}^{-1}$. Full details are not yet available.

The $\mathrm{I}^{1} \Sigma^{-}$state had previously been known only from its perturbation of the $\mathrm{A}^{1} \Pi$ state (see table 54a). A number of the perturbations caused by the I state have been assembled by Schmid and Gerö [225, 234b], Kovács [135], and from more recent measurments of the $\mathrm{B}^{1} \Sigma^{+}-\mathrm{A}^{1} \mathrm{II}$ Angstrom bands by Deutsch and Barrow [52]. These authors all assumed that the $v=0$ level of the I state perturbed $A^{1} \Pi, v=1$. The above measurements [100] suggest that it is probably the $v=1$ level of the I state that is involved.

Earlier estimates of $B_{e}$ of about $1.48 \mathrm{~cm}^{-1}$ 
implied a value of $\alpha_{e}$ of about 0.046 which is rather large for any state of $\mathrm{CO}$. [But the $b$ state has an $\alpha_{e}$ of 0.042 or 0.033 ]. Deutsch and Barrow [52], by assuming the value of $\alpha_{e}$ as 0.018 $\mathrm{cm}^{-1}$ which is similar to other states having the configuration $\pi^{3} \sigma^{2} \pi^{*}$, have derived a term value for the electronic state and vibrational and rotational constants. These values, adjusted by raising their $v$ values by one unit, are listed in table 1. The state lies at about $65630 \mathrm{~cm}^{-1}$.

A summary of the observed $I^{1} \Sigma^{-}$perturbations is given below:

\begin{tabular}{c|r|r|l}
\hline$v\left(\mathrm{~A}^{1} \Pi\right)$ & $v\left(\mathrm{I}^{1} \Sigma^{-}\right)$ & $J$ & $\mathrm{~T}_{v-0}\left(\mathrm{I}^{1} \Sigma^{-}\right)$ \\
& & & \\
\cline { 2 - 3 } 1 & 1 & 35 & 66678 \\
2 & 2 & 7 & 67704 \\
3 & 4 & 40 & 69699 \\
4 & 5 & 23 & 70667 \\
5 & 7 & 44 & 72547 \\
6 & 8 & 30 & 73459 \\
8 & 11 & 34 & 76080 \\
9 & 12 & 13 & 76915 \\
11 & 15 & 16.5 & 79360 (ref. 234b) \\
\hline
\end{tabular}

All data points but the last are those of Deutsch and Barrow [52]. (Term values are in $\mathrm{cm}^{-1}$ ).

\subsection{Unidentified Bands}

A. Herman [95] has found two new groups of triplets attributed to $\mathrm{CO}$, which had earlier been found in comets, using as source a 90-cm long discharge tube with carbon electrodes, filled with xenon to a pressure of $10 \mathrm{~mm}$ of $\mathrm{Hg}$. The two groups of triplets have different structure. Group (a) is red-degraded as is evident even under low dispersion, group (b) has branches which look like lines (as may be seen in fig. 1 of ref. 95). (See table 35e.) No further details are given.

B. Johnson [118] has observed five doubleheaded red-degraded bands together with other bands belonging to $\mathrm{CO}^{+}$. They are described as similar to the $\mathrm{A}^{2} \Pi_{i}-\mathrm{X}^{2} \Sigma^{+}$comet tail bands, but with different separation, and each head is not a close doublet. These are given in table $35 \mathrm{j}$.

C. Barbier [11] has observed 26 bands in the night sky (5200-3200 $⿱$ ) which were attributed to a new transition to the ground state of CO. 'The upper state is at $21962 \mathrm{~cm}^{-1}$, with $\omega_{e}^{\prime}=2610 \mathrm{~cm}^{-1}$ and $\omega_{e} x_{e}^{\prime}=23.8 \mathrm{~cm}^{-1}$. Pearse [186] has questioned this assignment since $\omega_{e}^{\prime} \gg \omega_{e}^{\prime \prime}$. 'The identity of these bands as belonging to $\mathrm{CO}$ is highly questionable.

D. Huffman et al. [107] have observed numerous unclassified bands (table 35f) in their study of the absorption coefficients of $\mathrm{CO}$ between 1000-600 $\AA$. 'These are in addition to many previously observed Rydberg states, and other bands seen by Tanaka [254] and Henning [91].

\subsection{9. $\mathrm{P}, \mathrm{Q}, \mathrm{R}, \mathrm{S}, \mathrm{T}-\mathrm{X}^{1} \Sigma^{+}$Tanaka Systems (800-630 ̊) R}

Among numerous absorption bands which make up several Rydberg series, Tanaka [254] observed five new progressions with roughly constant frequency difference. These progressions, representing transitions from the ground state to the P, Q, R, S, and T states, are listed in table 26 . Weissler et al. [270] have observed the $\mathrm{P}$ state and the $\mathrm{S}$ (or $\mathrm{T}$ ) state by photoionization. Kaneko [124] has observed these in electron impact.

\subsection{Rydberg Series (940-630 A)}

Rydberg series are of special interest because their convergence limits give the ionization potentials of the molecule. Absorption measurements have been reported of such series whose convergence limits coincide with the three spectroscopically observed states of $\mathrm{CO}^{+}$. Recently, series converging to what appears to be a new state of $\mathrm{CO}^{+}$have been observed [42]. The series converging to the $\mathrm{B}, \mathrm{A}$, and $\mathrm{X}$ states of $\mathrm{CO}^{+}$are given, respectively, in tables 27,28 , and 29. The observed Rydberg states of $\mathrm{CO}$ are most likely ${ }^{1} \Sigma^{+}$or ${ }^{1} \Pi$, though no fine structure analyses hare yet confirmed the tentative assignments. Details of their possible electronic structure have been given in section 2. The spectroscopic obserrations are reviewed below.

A number of bands which reportedly belong to Rydberg series have been observed under low resolution by Henning [91] and Anand [2]. Lncertainties in the measurements make their series limits unreliable. Henning [91] observed 2.2 bands in absorption, including 10 which formed two progressions having nearly equal frequency difference $\left(\simeq 1550 \mathrm{~cm}^{-1}\right)$. The positions of these broad bands are uncertain by $\pm 50 \mathrm{~cm}^{-1}$. Anand [2] observed six headless bands in emission, and in the same spectral region $(900-\$ 50 \AA)$ saw a series of four bands in absorption. Both series were 
fitted by a Rydberg formula with a common limit of $14.5 \mathrm{eV}$, which falls between the term values of vibrational levels of $\mathrm{CO}^{+}, \mathrm{X}^{2} \Sigma^{+}$. Henning's bands are listed in table $35 \mathrm{~g}$. Anand's are listed in table $35 \mathrm{~h}$.

Tanaka [254], using a 3-m focal length grazing incidence vacuum spectrograph, observed numerous bands, including most of those reported by Henning, as well as the $F$ and $G$ bands of Hopfield and Birge [104]. A band at 783.1 $\left(127,698 \mathrm{~cm}^{-1}\right)$, reported to be very diffuse by Henning, was seen under higher dispersion to have three close heads, each being sharp and degraded to the red. Tanaka found two sets of absorption bands of equal intensity, one sharp and one diffuse, each forming a Rydberg series labeled the " $\beta$ " series and converging to a common limit at $630.12 \AA=19.675 \mathrm{eV}$. The sharp series in the region 700-620 $\AA$ can be represented by the formula

$$
\sigma=158692-\frac{\mathrm{R}}{(n-1.68)^{2}}, n=5, \ldots, 12 .
$$

More recently, Huffman, Larrabee, and Tanaka [107] have observed the $n=4,0-0$ terms of the " $\beta$ " sharp and diffuse Rydberg series in their study of absorption coefficients of $\mathrm{CO}$ in the region 1000-600 ̊. (See also table 27.)

Another Rydberg series labeled " $\alpha$ " was found [by Tanaka] to span the region $800-730 \AA$. Its convergence limit was at $749.74 \AA=16.536 \mathrm{eV}$. The " $\alpha$ " series can be represented by the formula

$$
\sigma=133380-\frac{\mathrm{R}}{(n-1.69)^{2}}, n=5, \ldots 9 .
$$

The " $\beta$ " series includes transitions from the $\mathrm{X}^{1} \Sigma^{+}$state of $\mathrm{CO}$ to electronic states which converge to the $\mathrm{B}^{2} \Sigma^{+}$state of $\mathrm{CO}^{+}$. A diffuse band always appeared on the short wavelength side of each sharp band. The " $\alpha$ " series converges to the $\mathrm{A}^{2} \Pi$ state of $\mathrm{CO}^{+}$. This latter series is strongly overlapped by other bands. Both " $\beta$ " and " $\alpha$ " series show vibrational structure which is close to that of the ionic states which are their series limits. Tanaka [254] tentatively ascribed the sharp and diffuse series to $\left({ }^{1} \Sigma^{+}\right)-\mathrm{X}^{1} \Sigma^{+}$and $\left({ }^{1} \Pi\right)-{ }^{1} \Sigma^{+}$transitions, respectively.

Takamine, Tanaka, and Iwata [253] have observed two Rydberg series converging to $884.73 \AA$ and $868.13 \AA$, respectively. These series are the $0-0$ and $1-0$ vibrational series whose convergence limit is the lowest ionization potential of $\mathrm{CO}$ :
$113029 \pm 32 \mathrm{~cm}^{-1}$ or $14.013 \pm 0.004 \mathrm{eV}$. This convergence limit is within the experimental error of that derived from the limits of the " $\beta$ " and " $\alpha$ " series and the origin of the $\mathrm{B}-\mathrm{X}$ system of $\mathrm{CO}^{+}$. (The values in eV quoted here differ from those originally given in ref. 253 because of a slight difference in conversion factor.) The series has been represented by a formula

$$
\sigma=113029-\frac{\mathrm{R}}{(n-1.88)^{2}}, n=6, \ldots, 14 .
$$

\subsection{1. $\mathrm{B}^{2} \Sigma^{+}-\mathrm{X}^{2} \Sigma^{+}$First Negative System of $\mathrm{CO}^{+}$ (3150-1800 ̊) R}

The $\mathrm{B}^{2} \Sigma^{+}-\mathrm{X}^{2} \Sigma^{+}$First Negative system of carbon, originally observed by Deslandres (see also ref. $127 \mathrm{a}$, p. 233), consists of an extensive group of single-headed, red-degraded bands. The upper state of this system is the highest lying state of $\mathrm{CO}^{+}$which has been observed spectroscopically, though a more energetic state has been tentatively identified from electron impact and photoionization measurements. Vibrational heads of the $\mathrm{B}-\mathrm{X}$ system are given in table 30 , origins in table 31. Rotational constants for the $\mathrm{X}^{2} \Sigma^{+}$and $\mathrm{B}^{2} \Sigma^{+}$states of $\mathrm{CO}^{+}$are given in tables 50 and 52 , respectively.

A number of bands observed by Schniederjost [ref. 127a, p. 213] were later correctly identified by Jevons [116] as belonging to Deslandres' system. Additional bands of this system were reported by Johnson [118] and Blackburn [26] who gave the first vibrational assignments. This system was extended to shorter wavelengths by Biskamp's report [24] of 22 new bands in an intense high frequency discharge in helium containing a trace of CO. The dispersion used was $8.8 \AA / \mathrm{mm}$ [wavelengths are uncertain to $\pm 0.05 \AA]$. A formula was fitted to the observed band heads:

$$
\begin{aligned}
\sigma=45651.5+\left(1695.6 v^{\prime}-24.25 v^{\prime 2}\right) \\
-\left(2196 v^{\prime \prime}-15.12 v^{\prime \prime 2}\right)
\end{aligned}
$$

Deviation from this formula of levels with $v^{\prime}=7$ suggested [24] perturbation of this level as due to crossing of the ${ }^{2} \Sigma$ states.

Early fine structure measurements on lower vibrational levels of both states did not provide reliable rotational constants because of overlapping lines and poor resolution. These include studies by Coster, Brons, and Bulthuis [45] and by Schmid [237]. The latter observed measurable 
spin splitting only for high rotational quantum number, typically about $30-34$. The $3-5$ band, however, showed doubling (about $1.3 \mathrm{~cm}^{-1}$ width) for $\mathrm{P}(23)$ and $\mathrm{R}(24)$.

The rotational analysis of the $0-0$ band by Schmid and Gerö [222] established the ground state as the common lower state of the Comet Tail and First Negative bands.

Rao [202a] has made a comprehensive study of the $\mathrm{B}-\mathrm{X}$ system of $\mathrm{CO}^{+}$under high resolution which has provided the best values of molecular constants of both states, band origins, rotational constants, and spin doubling coefficients. [For the latter see table 53.] Previously Woods [278] had reported values of $\left|\gamma^{\prime}-\gamma^{\prime \prime}\right|$ for the $B$ and $X$ states which are about half those of Rao.

Rao [202a] has given the fine structure of the $0-0,0-1,0-2,0-3,0-4,1-2,1-3,1-4,1-5,2-3$, $2-4,2-5,2-6,3-5$, and $4-7$ bands. Observations were made viewing a hollow cathode discharge with a $21-\mathrm{ft}$ focal length grating spectrograph in fourth order. Six branches are possible for a ${ }^{2} \backsim-{ }^{2} \Xi$ transition, with each line of the $\mathrm{P}$ and $\mathrm{R}$ branches split into a triplet by the selection rule $\Delta \mathrm{J}=0, \pm 1$. The satellite branches ${ }^{\mathrm{P}} \mathrm{Q}_{12}$ and ${ }^{\mathrm{R}} \mathrm{Q}_{21}$ were very weak except at very low $N$. The bands consist of a doublet $\mathrm{P}$ branch and a doublet $\mathrm{R}$ branch, with doublet splitting resolved only for higher $\mathrm{N}$ values.

\subsection{2. $\mathrm{A}^{2} \Pi_{i}-\mathrm{X}^{2} \Sigma^{+}$Comet Tail System ${ }^{8}$ of $\mathrm{CO}^{+}$ (7200-3080 ̊) $\mathrm{R}$}

Important characteristics of comet tail spectra are bands of carbon compounds. Among these are found the strongest bands of $\mathrm{CO}^{+}, \mathrm{A}^{2} \Pi_{i}-\mathrm{X}^{2} \Sigma^{+}$, which were first discovered in these celestial sources [189], and shortly thereafter weakly produced in low pressure Geissler discharges in $\mathrm{CO}$ by Fowler [64]. 'These bands which extend from the UV through the visible region have two double heads degraded to the red. Each band shows eight branches (out of a possible 12), four originating from each II doublet component. The other four are not resolved because of unresolved spin splitting of the $\mathrm{X}^{2} \Sigma$ state. Rao [202b] has found that previously used $v^{\prime}$ numbering should be lowered by three units. Perturbations of levels $A^{2} \Pi_{i}, v=5$ and 10 indicate crossing by the ground state. Table 32 lists the observed

${ }^{8}$ Farlier referred to as the Third Negative bands of carbon.
$\mathrm{A}-\mathrm{X}$ band heads; precise origins are listed in table 33. Rotational constants for the $\mathrm{X}$ and $\mathrm{A}$ states of $\mathrm{CO}^{+}$are given in tables 50 and 51 , respectively.

Merton and Johnson [160], Johnson [118], and more recently Asundi [6] have observed bands of this system in a discharge through heliun with a very small partial pressure of $\mathrm{CO}\left(\sim 10^{-4} \mathrm{~mm}\right)$. Baldet [9] observed four heads of each of 40 bands (in the region 6400-3080 $\AA$ ) produced by electron bombardment of $\mathrm{CO}$ in the most extensive single observation of this system. He drew a Fortrat parabola for the eight observed branches of the band at $3997 \AA$, but the more extensive rotational analysis of many bands to which he alluded has not been published.

Birge [21] showed that the First Negative and Comet Tail bands had a common lower state. His vibrational assignments, as given in an early review by Herzberg [97], were based on a fit to all of Baldet's data with neglect of a weak band at $5281 \AA$. This analysis did include three comparably weak bands at $6400 \AA, 5800 \AA$, and $6000 \AA$, whose intensities were estimated as $1 / 2$ by Baldet. These were assigned as $0-0,1-0$, and 2-1 transitions. Rao [202b] did not see these weak bands, nor have any B-A bands been observed with $v^{\prime \prime}$ values corresponding to the above assignments. In addition, the above quantum assignments gave abnormal Condon parabolas. By lowering the $v^{\prime}$ assignments by three units and omitting the four weak bands (listed in table $35 \mathrm{i})$, these difficulties were eliminated [202b].

Much work has been devoted to fine structure analysis of lower lying vibrational levels. Blackburn [25] reported seeing the Comet Tail bands under high resolution, but gave no quantitative data. Sometime later Coster, Brons, and Bulthuis [45] observed the fine structure of the 2-0, $3-0,4-0$, and $5-0$ bands produced in a low pressure discharge in flowing CO. Relative accuracy for sharp lines was claimed to be $0.002 \AA$. 'The ${ }^{2} \Pi_{i}$ state was found to be in Fund's coupling case (a) for low rotational quantum numbers. Schmid and Gerö [222] have given the fine structure of the $0-0,1-0,1-1,2-1,3-2$, and $4-2$ bands. Rotational analyses of many more bands have been given by Buithuis $[34 \mathrm{a}, 35]$ who has published details only of the $6-0$ band and the most intense branches of the $7-0, S-1,9-1,10-1$, 10-2, and 11-2 bands. Perturbations of the upper level were found in the 10-1 and 10-2 bands. 
The fine structure of the $0-2,0-3$, and $0-4$ bands has been given by Rao [202b] who, in addition, reduced previous data $[34,45,222]$ to evaluate rotational constants for the $\mathrm{A}^{2} \Pi_{i}$ state. Accuracy of $\pm 0.00025 \mathrm{~cm}^{-1}$ is claimed. The following formula was obtained:

$$
\begin{gathered}
\mathrm{B}_{v}^{\prime}=1.58940-0.01942\left(v^{\prime}+\frac{1}{2}\right), \quad \mathrm{D}_{v}^{\prime} \sim 6.60 \times 10^{-6} \\
\mathrm{~cm}^{-1}(\mathrm{est}) .
\end{gathered}
$$

An average value of $-117.5 \mathrm{~cm}^{-1}$ was derived for the coupling constant $\mathrm{A}$ (which is negative because the A state is inverted). A useful comparison of the branch designations used by previous authors can be found in Rao's paper [202b].

\subsection{3. $\mathbb{B}^{2} \Sigma^{+}-\mathbf{A}^{2} \Pi_{i}$ Baldet-Johnson Intercom- bination System of $\mathrm{CO}^{+}(4240-3310 \AA) \mathrm{V}$}

This system of double double-headed, violetdegraded bands has been much less extensively studied than the other two known transitions for $\mathrm{CO}^{+}$. Spin splitting of the upper state is observable; for the lower state it is negligible. Vibrational quantum numbering is based on the work of Rao [202b]. Observed band heads are given in table 34 .

Merton and Johnson [160] mentioned a number of faint violet-degraded bands among their A-X Comet Tail bands and similar in appearance. Johnson [118] reported the band heads for the $0-1,1-2,0-0,1-0,2-0$, and 3-0 transitions. Several additional bands were found which have not been identified (table 35j). Meanwhile,
Baldet [8] had previously reported the $0-1,0-0$, and 1-0 transitions from electron bombardment of low pressure CO.

Baldet [10] mentioned high dispersion measurements of the $0-1,0-0$, and $1-0$ bands, but his fine structure analysis has not been published. Bulthuis [34b] has given the rotational analysis of the $0-0$ and $0-1$ bands. These bands extended to moderate $J(\sim 25)$, were very weak, and were overlapped by the much stronger $\mathrm{A}-\mathrm{X}$ Comet Tail bands. The bands were observed in a hollow cathode discharge. The spin splitting of the $\mathrm{B}^{2} \Sigma$ level was observable, and Bulthuis concluded that the spin splitting in the $\mathrm{B}-\mathrm{X}$ band for higher $J[34 b]$ is due essentially to the B state. Rotational constants for each ${ }^{2} \Pi$ sublevel were obtained which, when averaged, are in reasonable agreement with more recent results of $R_{a o}$ [202b]. For higher $J$ the doublets $P_{2}-Q_{12}$ and $R_{1}-Q_{21}$ are clearly separated, but the doublets $R_{12}-Q_{2}$ and $\mathrm{P}_{21}-\mathrm{Q}_{1}$ show only one component even for the highest $J$ values $(\sim 25)$.

Recently, Rao and Sarma [205] have summarized the work on all three observed transitions for $\mathrm{CO}^{+}$stressing the revision of $v^{\prime}$ numbering for some $\mathrm{B}^{2} \Sigma-\mathrm{A}^{2} \Pi_{i}$ Baldet-Johnson bands, and changes in branch designation because earlier fine structure analysis incorrectly labeled the $\mathrm{A}$ state as ${ }^{2} \Pi$, instead of inverted. Making use of the value for the spin splitting constant $\gamma=0.0192$ for $B^{2} \Sigma$, $v=0$ and $\left|\gamma^{\prime}-\gamma^{\prime \prime}\right|$ obtained previously [202a], they obtained values of $\gamma$ for the $\mathrm{X}$ state which are given in table 53. Blending of branches of the intercombination bands prevented accurate determination of rotational constants.

\section{Perturbations}

A number of states of $\mathrm{CO}$ and $\mathrm{CO}^{+}$undergo perturbations, most notably the $\mathrm{A}^{1} \Pi$ and $b^{3} \Sigma^{+}$. The A state has the greatest number of perturbations; the $b$ state suffers perhaps the most severe disruptions in its rotational structure.

For $\mathrm{CO}$, the identity of most perturbing states is known. The existence of the $\mathrm{I}^{1} \Sigma^{-}$state, not directly observed until recently, was revealed from itsinteractions with the $\mathrm{A}^{1} \Pi$ state. Supposed predissociations of the A state $[227,74]$, indicated by intensity drops in the band fine structure, are not real. However, perturbations may occur at these positions. Table 54 summarizes the data on reported maximum perturbations, some of which are uncertain.

This section which supplements table 54 is concerned only with the reported rotational perturbations. Multiplet splitting and $\Lambda$-doubling are discussed in section 3 ; predissocations, a special case of perturbations, are discussed in section 5 .

\section{1. $\mathrm{A}^{1} \Pi$ State}

$$
\text { a. } v=0
$$

Stepanov [244] has determined the matrix elements of interaction of ${ }^{3} \Sigma_{J=N}$ with ${ }^{1} \Pi_{b}$ (in inter- 
mediate coupling between cases (a) and (b)), and has applied the results to perturbations of $A^{1} \Pi$, $v=0, J=9,12,16$. By using the data of Gerö [224a, 71], he obtained numerical values of the matrix elements which were found to be functions of $\mathrm{J}$, and not constant as had been found by Budo and Kovács [33]. The almost linear increase with $J$ led to the conclusion that the $A^{1} \Pi$ state was in intermediate coupling close to case (b), as had been previously established (see sec. 3.1). Stepanov [245] has also mentioned the importance of including the distorting affect of a third level at the common point of intersection for low $\mathrm{J}$.

Coster and Brons [44] have also reported a perturbation affecting all branches at $\mathrm{J}=1$; the perturber is $\Pi$ or $\Delta$ since both $\Lambda$-components are affected together, most probably ${ }^{1} \Pi$ since the perturbation is already large at $\mathrm{J}=1$. This perturbing ${ }^{1} \Pi$ state has not been observed directly. In addition, all lines below $J=16$ showed deviations of $6-10 \mathrm{~cm}^{-1}$; most of these being due to crossing by ${ }^{3} \Sigma$ levels. The ${ }^{3} \Sigma$ term value was estimated at $64815 \mathrm{~cm}^{-1}$; from the data of Herzberg and Hugo [101] $e^{3 \Sigma^{-}}, v=1$ was found at 64803 $\mathrm{cm}^{-1}$. For high J (presumably $>30$ ) [44], a ${ }^{3} \Pi$ perturbation is mentioned.

Crawford [50] has studied the Zeeman effect in the B-A Angstrom bands. [His fig. 5 shows the Zeeman pattern for the $P(1)$ and $R(1)$ triplets of the $0-0$ band at $4511 \AA$ and also the asymmetry of the $\mathrm{Q}$ doublets at low $\mathrm{J}$ for the $0-3$ band at $5610 \AA$.$] He has tabulated a number of magnet-$ ically sensitive lines which show large Zeeman patterns. These normally occur near points of large rotational perturbations. For the $0-0$ band this includes $\mathrm{Q}(25)$ and probably others. (See sec. 4.1, $\mathrm{b}$ to $\mathrm{d}$ for additional magnetically sensitive lines.) Crawford described the appearance of the 0-0 B-A band as having weak and fragmentary extra branches (see sec. 3.2 ; note particularly the same difficulty of Jasse [115]).

Watson [268, 269] has reported additional faint lines for the $0-0 \mathrm{~B}-\mathrm{A}$ bands including the occurrence of extra lines at the perturbations. Some of the $\mathrm{P}, \mathrm{Q}$, and $\mathrm{R}$ lines at the perturbation of $\mathrm{J} \sim 28$ become sharp doublets. The Zeeman pattern indicated that the perturber was not a singlet state, and was assumed to be $d^{3} \Pi_{i}$ (now known to be $d^{3} \Delta_{i}$ ). Extra lines appear for $\mathrm{J}=8,10$, and 17 .

The perturbed lines show large irregular Zeeman patterns at medium and high J values. Neighboring lines are apparently insensitive to the magnetic field. 'The perturbing state is assumed to be case (a) ${ }^{3} \Pi$. Watson [269] has given a table of Zeeman patterns of the perturbed band lines in the $0-0$ B-A band.

$$
\text { b. } v=1
$$

At J 0 Rosenthal and Jenkins [216] found a perturbation in all branches which they ascribed to $a^{3} \Pi, v=1$. Schmid and Gerö [225] believed, however, that the perturbing state was ${ }^{1} \Pi$, an unobserved state.

Coster and Brons [43] have found the following perturbations in the $0-1 \mathrm{~B}-\mathrm{A}$ band: $\mathrm{R}(26), \mathrm{P}(26)$, $\mathrm{Q}(23), \mathrm{Q}(26), \mathrm{Q}(29)$, and $\mathrm{Q}(35)$, with extra lines corresponding to $\mathrm{R}(26)$ and $\mathrm{Q}(29)$. These seem to include the $a^{\prime 3} \Sigma^{+}$perturbations as well as ${ }^{1} \Sigma^{-}$ perturbations reported elsewhere $[262,225]$. The observed deviations do not exceed $2 \mathrm{~cm}^{-1}$, with the largest deviations accompanied by intensity reductions. Two additional $\Sigma$ perturbations are found at $\mathrm{Q}(25)$ and $\mathrm{Q}(35)$ which may also be due to the ${ }^{1} \Sigma^{-}$state.

Read [209] has observed a perturbation at $\mathrm{J} \sim 26$ in the 1-0 A-X band. Gerö [74] reported a ${ }^{3} \Sigma^{-}$ perturbation at $J=44$ in another $A-X$ band.

Gerö [71] has found small perturbations at small $J$ in $v=1$ with both $\Lambda$-components affected the same.

Tschulanowsky and Stepanow [262] observed perturbations in $\mathrm{A}-\mathrm{X}$ bands, including the appearance of extra lines. In all cases the $\mathrm{P}$ and $\mathrm{Q}$ branches were perturbed differently and perturbations result from interaction with the $e^{3} \Sigma^{-}$state. For the 1-5 A-X band an extra line was incorrectly attributed to $\mathrm{Q}(2 \mathrm{~S})$ and should be rerised as follows: $\mathrm{Q}(2 \mathrm{~S})=55571.07, \mathrm{Q}(29)=55554.84$, with the extra line for $J=29$ at $55559.10 \mathrm{~cm}^{-1}$. A ${ }^{1}$ 工 term perturbs both $\mathrm{J}=26$ and 35 . An additional perturbation at $J=26$ [44] is due to one component of a ${ }^{3} \Sigma$ state.

Crawford [50] has listed as magnetically sensitive lines Q(23) and Q(24) in the 0-1 B-A band. Near the perturbation at $\mathrm{J} \sim 28$ in this band Watson $[268,269]$ found that sereral $P, Q$, and $R$ lines appeared as doublets.

McCulloh [157a] reported a perturbation of the lower state in the $0-1 \quad \mathrm{~B}-\mathrm{i}$ band for $\left({ }^{13} \mathrm{O}^{10}\right.$. Perturbed lines include $\mathrm{P}(16), \mathrm{Q}(13), \mathrm{Q}(19)$, and $R(16)$. The perturbing state is ${ }^{3}=+$ with $B-1.1 \%$ $\mathrm{cm}^{-1}$. (This is the isotopic analog to what Conter and Brons [43] found.) 


$$
\text { c. } v=2
$$

Rosenthal and Jenkins [216] observed perturbations at $J=25,29$, and 33 in the $0-2 \mathrm{~B}-\mathrm{A}$ band. Extra lines appeared in the $P$ and $R$ branches for $\mathrm{J}=25$ and 33 . Tschulanowsky and Stepanow [262] saw the same perturbations in the $\mathrm{A}-\mathrm{X}$ bands including extra lines in the $\mathrm{P}$ branch for $\mathrm{J}=25$ and 26. The perturbing state is $e^{3} \Sigma^{-}, v=4$ (from the data of Herzberg and Hugo [101]). From perturbations [44], the term value of $67953 \mathrm{~cm}^{-1}$ is obtained.

Gerö [73] observed doubling of the $Q(7)$ line in $\mathrm{A}-\mathrm{X}$ bands. The perturber is ${ }^{1} \Sigma^{-}$. Deutsch and Barrow [52] observed this perturbation in the $\mathrm{B}-\mathrm{A}$ bands, and have tabulated the perturbations caused by the $\mathrm{I}^{1} \Sigma^{-}$state for all vibrational levels of the $A^{1} \Pi$ state. Crawford [50] gives the following magnetically sensitive lines in the $0-2 \mathrm{~B}-\mathrm{A}$ band: $\mathrm{P}(25), \mathrm{P}(26), \mathrm{P}(34)$, and $\mathrm{P}(35)$.

$$
\text { d. } v=3
$$

Rosenthal and Jenkins [216] reported perturbations at $\mathrm{J}=28,31$, and 34 in $\mathrm{B}-\mathrm{A}$ bands. Coster and Brons [44] showed that these levels are crossed by the $a^{\prime 3} \Sigma^{+}$state. The estimated term value for the perturber is $69459 \mathrm{~cm}^{-1} ; a^{\prime 3} \Sigma^{+}, v=12$ is found at $69602 \mathrm{~cm}^{-1}$ from the data of Herzberg and Hugo [101]. Coster and Brons [44] found another small perturbation of $Q(38)$ which is due to the ${ }^{1} \Sigma^{-}$state.

Gerö [74] found $J=50$ perturbed by the $e^{3} \Sigma^{-}$ state. Tschulanowsky and Stepanow [262] have seen the ${ }^{3} \Sigma$ perturbation in the $\mathrm{A}-\mathrm{X}$ bands. In the $3-7$ band extra lines are found for $\mathrm{Q}(27)$ and $\mathrm{Q}(28)$. The extra line reported for $\mathrm{Q}(29)$ may not be real, and cannot be assigned to $J=28,29$, or 30. Crawford [50] includes the $\mathrm{Q}(28)$ line as magnetically sensitive in the $0-3 \mathrm{~B}-\mathrm{A}$ bands. Deutsch and Barrow [52] found $\mathrm{I}^{1} \Sigma^{-}$perturbing $\mathrm{J}=40$.

McCulloh [157a] found the maximum perturbation at $\mathrm{Q}(21)$ in the $0-3 \mathrm{~B}-\mathrm{A}$ band of $\mathrm{C}^{13} \mathrm{O}^{16}$. $\mathrm{P}(21)$ was unperturbed. Either the ${ }^{3} \Sigma^{+}$state or ${ }^{1} \Sigma^{-}$is responsible for the perturbation; the proper assignment can only be established from examination of high rotational levels.

$$
\text { e. } v=4
$$

Coster and Brons [44] have found a perturbation at $\mathrm{J} \sim 31$ in the $0-4 \mathrm{~B}-\mathrm{A}$ band, the reality of which is disputed by Gerö [73]. Another perturbation at $J=34,37$, , is due to $e^{3} \Sigma^{-}$. The estimated term value of ${ }^{3} \Sigma$ is $70878 \mathrm{~cm}^{-1}$; from Herzberg and Hugo [101], one obtains $e^{3} \Sigma^{-}, v=7$ at 70966 $\mathrm{cm}^{-1}$.

Gerö $[73,71]$ has reported a ${ }^{3} \Sigma^{+}$perturbation at $J \sim 0$ and two others at $J=23$ and 27 . The one at $J=23$ is due to ${ }^{1} \Sigma^{-}$[52]. Asundi [5], in addition, observed a very small perturbation for $J=18$. Gerö [73] has reviewed the work on a number of these perturbations and gives the following identifications: $J \sim 0,{ }^{3} \Sigma^{+} ; \quad J=23,{ }^{1} \Sigma^{-} ; \quad J=37,{ }^{3} \Sigma^{-}$. Additional perturbations include $J=46,{ }^{3} \Sigma^{+}$; $J=50,{ }^{3} \Pi$.

$$
\text { f. } v=5
$$

Gerö [73] has referred to close crossing of ${ }^{3} \Sigma^{+}$ and ${ }^{3} \Pi$ states which overlap perturbations of $A$, $v=5$. The first perturbation is at $\mathrm{Q}(30)$ in $\mathrm{A}-\mathrm{X}$ bands, and is due to ${ }^{3} \Sigma^{+}$. The last perturbation of this pair of overlapping states is at $R(35)$, $\mathrm{Q}(36)$, and $\mathrm{P}(37)$, due to ${ }^{3} \Pi$. $\quad \mathrm{A}^{1} \Sigma^{-}$perturbation is found at $Q(44)[73,52]$, and the onset of $a^{3} \Sigma^{-}$ perturbation at $R(50)$.

$$
\text { g. } v=6
$$

Gerö [73] has observed overlapping ${ }^{3} \Pi$ and ${ }^{3} \Sigma$ perturbations near the heads of $\mathrm{A}-\mathrm{X}$ bands. An additional perturbation was observed at $\mathrm{Q}(52)$. Since the other branches were not followed to such high quantum numbers the nature of the perturbing state could not be determined with certainty, and was assumed by Gerö to be ${ }^{3} \Pi$. Several additional perturbations at $J=29,30$ have also been found $[73,52]$.

Onaka [182] later unraveled the structure near the head in his observations of the 6-13 and 6-14 A-X bands. Details of the 6-14 band are not given. A perturbation near $\mathrm{J}=0$ which acts on all branches, indicates a $\Pi$ or $\Delta$ perturber with $\mathrm{B}_{v}$ larger than that of the A state. (A similar perturbation has been found of $A, v=0$ by Coster and Brons [44], indicating the possibility of a common state perturbing both the 0 and 6 levels.) Several extra lines are observed for $v=6$, which include $\mathrm{P}(7), \mathrm{R}(7), \mathrm{Q}(11), \mathrm{P}(13), \mathrm{Q}(13)$, and $\mathrm{Q}(16)$. The perturbations observed are: $J^{\prime}=7 \sim S, 11$, 14,16 in $Q$; and $J^{\prime} \sim 0$, all branches.

$$
\text { h. } v=7
$$

Read [209] has observed perturbations of $R(25)$, $\mathrm{Q}(27)$, and $\mathrm{P}(27)$ in the $7-1 \mathrm{~A}-\mathrm{X}$ band. Another 
perturbation at $J=29$ was designated as ${ }^{3} \Sigma^{-}$by Gerö [73]. In addition, Gerö found a ${ }^{3} \Sigma^{+}$perturbation at $J=26$, and a ${ }^{3} \Pi$ perturbation at $J=39$. Gerö [74] mentions a sudden intensity drop from $R(46)$ to $R(47)$ in the $7-16 \mathrm{~A}-\mathrm{X}$ band with no simultaneous line displacement.

$$
\text { i. } v=8
$$

Gerö [73] found the following perturbations in A-X bands: $J=18,{ }^{3} \Sigma^{+} ; J=27,{ }^{3} \Pi ; J=34$ [52], ${ }^{1} \Sigma^{-}$. Gerö [74] mentions the following sudden intensity drops (no line displacement)

$$
\begin{gathered}
\text { 8-16, } \mathrm{A}-\mathrm{X} R(35)>P R(36) \\
8-17, \mathrm{~A}-\mathrm{X} \mathrm{R}(35)>P R(37) ; \mathrm{R}(36) \text { over- } \\
\text { lapped. }
\end{gathered}
$$$$
\text { j. } v=9
$$

Gerö [73] found a ${ }^{1} \Sigma^{-}$perturbation at $J=13$. (See also ref. 52.) At $J=37, a^{3} \Sigma^{+}$perturbation is expected [74]. Gerö [74] lists the following intensity drops unaccompanied by line displacements:

$\mathrm{A}-\mathrm{X}$

$9-17, \mathrm{P}(22)>\mathrm{P}(23) ; \mathrm{R}$ branch overlapped

$9-18, \mathrm{P}(22)>>\mathrm{P}(23) ; \mathrm{R}(19)>>\mathrm{R}(22)$; $\mathrm{R}(20), \mathrm{R}(21)$ overlapped; $\mathrm{Q}(27)>>$ $\mathrm{Q}(28) ; \mathrm{P}(28)>\mathrm{P}(29)$

$9-19, \mathrm{P}(22)>\mathrm{P}(23) ; \mathrm{R}(19)>>\mathrm{R}(21) ; \mathrm{R}(20)$ overlapped

9-20, $\mathrm{P}(23)$ and $\mathrm{R}$ branch overlapped; $\mathrm{Q}(27)$ $>\mathrm{Q}(28)$.

\section{k. $v=10$}

Gerö [73] has studied numerous perturbations of the $A^{1} \Pi$ state in the $A-X$ bands. It is determined that for $v^{\prime}=10$, at $\mathrm{J}=16$ and 18 , there are perturbations of $\mathrm{P}(17)$ and $\mathrm{Q}(18)$ by ${ }^{3} \Sigma^{-}$. The strongest perturbations are overlapped by the ${ }^{3} \Sigma^{+}$state. The branch lines, however, are of normal intensity beyond the perturbed level and can be followed to $J=34$. At $J=32$ there is a ${ }^{3} \Pi$ perturbation.

$$
\text { 1. } v=11
$$

Gerö [74] found a perturbation of $\mathrm{J}=16$ by ${ }^{3} \Pi$ and ${ }^{1} \Sigma^{-}$.

$$
\text { m. } v=12
$$

Gerö [74] found a perturbation of $\mathrm{J}=24 \mathrm{by}{ }^{3} \Sigma^{+}$.

$$
\text { n. } v=13
$$

Gerö [74] has analyzed lines only up to $J=15$. He indicates a probable ${ }^{3} \Pi$ perturbation near that limit. For the bands with $v=11,12,13$ only one band was observed, so that localizing the perturbations is less certain than for the other bands.

\subsection{E'II State}

In the 0-0 E-X band Tilford et al., [257] observed a perturbation at $\mathrm{J}^{\prime}=31$ in both $\mathrm{R}(30)$ and $\mathrm{P}(32)$. These lines appear as doublets, and, in addition, are anomalously more intense than their neighbors. A state of type ${ }^{1} \Sigma^{+}$is the suspected perturber.

\section{3. $a^{3} \Pi$ State}

Beer [16] has studied the perturbations of the $a^{3} \Pi, v=4$ level in the $0-4$ and $1-4$ bands of the $b^{3} \Sigma^{+}-a^{3} \Pi$ Third Positive bands. Eight observed crossing points are attributed to a perturbing ${ }^{3} \Sigma^{+}$ term for which $\mathrm{B}_{0}=1.31 \mathrm{~cm}^{-1}$ was estimated. The perturbing state, which lies at an energy of $55380 \mathrm{~cm}^{-1}$ above the reference lerel $\mathrm{X}^{1} \Sigma^{+}, v=0$, $J=0$, has been identified as $a^{\prime 3} \Sigma^{+}$[235]. From previous $b$ - $a$ studies by Dieke and Mauchly [54], and Gerö [75] it is shown that the $a^{3} \Pi, v=0,1,2$, and 3 terms are regular (unperturbed). Dieke and Mauchly [54] tried an analysis of the 0-4 Third Positive band, but could only conclude that strong perturbations were probably present. Beer found no perturbations of $v^{\prime \prime}=5$ from the $0-5$ and 1-5 bands. [In these bands the $b^{3} \Sigma, v=0$ and 1 levels are subject to strong and consecutive perturbations $[54,75]$. (See sec. 4.6.)

The eight perturbations are listed in table 54c. Numerous extra lines appear in the $0-4$ and $1-4$ bands.

Since the $a^{3} \Pi$ state is no longer in coupling case (a) for medium values of rotational quantum number [50], one can allow for violation of the selection rule for perturbations $\Delta \Omega=0, \pm 1$. In fact, in two places, the perturbations occur with $\Delta \Omega=2$. The absence of a perturbation at the ninth crossing point where $\Delta \Omega=3$ results from the rules for intermediate coupling. From the perturbations, $B \sim 1.31 \mathrm{~cm}^{-1}$ and $\sigma_{0} \sim 55,3 \varsigma 0 \mathrm{~cm}^{-1}$ (above $\mathrm{X}$ ) are calculated for the perturbing state. These values correspond closcly to those of the $a^{\prime 3} \Sigma^{+}, v=0$ level. The $0-6$ and $1-6$ bands should show the onset of perturbations sooner, but these were not analyzed due to insuficient light inten- 
sity. The lowest perturber level of $a^{\prime 3} \Sigma^{+}$is most certainly $v=0$, since $a^{3} \Pi, v=0,1,2$, and 3 show no perturbation. The Third Positive $0-6$ and 1-6 bands should show perturbations at $J \sim 47$. These have not yet been observed.

Beer's estimates of the constants for the $a^{\prime}$ state agree fairly well with the accurate determinations by Herzberg and Hugo [101].

\section{$4.4 d^{3} \Delta_{i}$ State}

Gerö and Szabó [82] have reported perturbations in all components of the $v=6$ term of the $d$ state in the $d^{3} \Delta_{i}-a^{3} \Pi$ Triplet bands at $\mathrm{J}=8, \mathrm{~J}=13-14$, and $\mathrm{J}=14-15$.

\section{5. $f^{3} \Sigma^{+}$State}

In a study of the $2980 \AA$ and $2670 \AA$ bands of the $f^{3} \Sigma^{+} \rightarrow a^{3} \Pi$ transition, Gerö [78] observed perturbations of the upper levels by the $v=0$ and $v=1$ levels of the $b^{3} \Sigma^{+}$state. The perturbations are seen in the $2670 \AA$ band at $N=13$ in the ${ }^{P} Q_{12}(\mathrm{~N}), \mathrm{P}_{2}, \mathrm{R}_{2}$, and ${ }^{R} Q_{32}$ branches. The $\mathrm{N}=13$ level for the other band also seems perturbed. A plot of $\frac{\Delta_{2} \mathrm{~F}(\mathrm{~N})}{4\left(\mathrm{~N}+\frac{1}{2}\right)}$ versus $\mathrm{N}$ indicates dependence on $N$ for small $N$; hence, a perturbation at $N \sim 0$ by another ${ }^{3} \Sigma^{+}$term. (See table 54e.) These bands had at one time been thought to originate from high vibrational levels of the $a^{\prime 3} \Sigma^{+}$state.

\section{6. $b^{3} \Sigma^{+}$State}

Dieke and Mauchly [54] have indicated that numerous large perturbations of the upper state of the $b^{3} \Sigma^{+}-a^{3} \Pi$ Third Positive bands cause the characteristic intensity fluctuations previously observed by Wolter [277]. The perturbations were ascribed to successive high vibrational levels of the ${ }^{3} \Sigma$ perturber, presumably $a^{3} \Sigma^{+}$. These are mixed with levels of the state $f^{3} \Sigma^{+}$. The first large perturbation in $v=0$ is at $\mathrm{N}=19$, hence the head region is not affected. However, there is no apparent regularity of lines in the middle of the bands. The perturbations result in clustering of lines in some places and gaps in others. Similar effects which are expected for lower $\mathrm{N}$ values near the head region for bands with $v^{\prime}=1$ would make the $v^{\prime}=1$ progression appear to belong to another system. In the $v^{\prime}=0$ progression for $v^{\prime \prime}=0$ to 4 , the last few lines in all branches showed perturbations of increasing magnitude with increasing $\mathrm{N}$ values, and the lines did not return to their regular sequence after perturbation. This indicates a $\Pi$ or $\Delta$ perturber. [Fig. 2 of ref. 54 shows the irregularly spaced triplets.] Deriations of a single perturbation exceed $50 \mathrm{~cm}^{-1}$ [53].

Gerö [72] determined numerous perturbations whose spacing decreased with increasing $\mathrm{N}$.

Gerö [75] observed the disrupted structure of the $1-0 \quad b-a$ band. Perturbations made it impossible to isolate branches below $\mathrm{N}=5$. Often lines did not follow in order of the quantum assignments. Additional perturbations were found for ${ }^{3} \Sigma$ levels $N=17,25,30,34$, and 40 . The perturber is the same ${ }^{3} \Sigma$ state which affects the $b^{3} \Sigma^{+}, v=0$ level.

The perturber would appear to be the $f^{3} \Sigma^{+}$state $[78,247,101]$, incorrectly identified previously as high vibrational levels of the $a^{\prime 3} \Sigma^{+}$state. (See further details on the $f-a$ transition in the section on electronic transitions.) The term values of the perturbing levels lie at $83744 \mathrm{~cm}^{-1}$ and $85969 \mathrm{~cm}^{-1}$.

\section{7. $\mathrm{A}^{2} \Pi_{i}$ State of $\mathrm{CO}^{+}$}

$$
\text { a. } v=\mathbf{5}
$$

The $A^{2} \Pi_{1 / 2}(v=5)$ level is perturbed by the $X^{2} \Sigma$ $(v=14)$ level [45]. In the 5-0, $\mathrm{A}^{2} \Pi_{1 / 2}-\mathrm{X}^{2} \Sigma^{+}$Comet Tail band, the perturbations include a number of successive extra lines in both the $Q_{1}$ and $Q_{2}$ branches (most of them being displaced towards higher frequency, one displaced towards lower frequency). The perturbing state is $\Sigma$. The perturbations occur for a large number of lines, because very similar $B_{v}$ values for both interacting levels cause both sets of levels to stay close together. At $J=14 \frac{1}{2}$ is the maximum perturbation of one $\Lambda$-component of the $\Pi_{1 / 2}$ level by the $\Sigma$ level with $J=N-\frac{1}{2} ;$ at $J=22 \frac{1}{2}$, the other $\Lambda$-component is perturbed by $\Sigma, J=N+\frac{1}{2}$; the $\Pi_{3 / 2}$ level is unperturbed. The perturbing $\Sigma$ level lies between $\Pi_{1 / 2}$ and $\Pi_{3 / 2}$ for low J. (See fig. 4 of ref. 45.) This, together with the fact that the peak perturbations lie so far apart, indicates a relatively slight angle between the potential curves of the interacting $\Pi$ and $\Sigma$ levels.

For small $J$, the $\Pi_{3 / 2}$ level is probably not perturbed. From extrapolation of data for the $2-0,3-0$, and $4-0$ bands, any displacement would not exceed several tenths of a $\mathrm{cm}^{-1}$. Both $\Lambda$-components of the $\Pi_{1 / 2}$ level are displacd about $2 \mathrm{~cm}^{-1}$ for low J. The perturber is $\mathrm{X}^{2} \Sigma^{+}$. From the two crossing points $\mathrm{X}_{v=14} \mathrm{~A}_{0=5}$, a value of $\mathrm{B}_{\Sigma} \approx 1.7 \mathrm{~cm}^{-1}$ is determined which is in fair agreement with the value for $\mathrm{X}^{2} \Sigma, v=14$. The 
extra lines are due to the $\mathrm{X}^{2} \Sigma(v=14)-\mathrm{X}^{2} \Sigma(v=0)$ transition. It seemed strange that perturbations were not also observed in the other bands considered here $(2-0,3-0$, and 4-0). 'This may be explained by the fact that the observed crossing of $I I$ and $\Sigma$ here indicates that the potential curves separate rapidly from one another, and have different $r_{e}$. The $\Sigma$ levels would appear to fall between the $\Pi$ levels for low $v$ and low $J$ so that a crossing with $\Pi_{3 / 2}$ is not expected till very high J. In fact, there appears to be a perturbation of $\Pi_{3 / 2}-\Sigma(4-0)$ for $\boldsymbol{J}>30_{1 / 2}$. (Probably due to $\mathrm{X}, v=10$ affecting the low energy $\Lambda$-component).

$$
\text { b. } v=10
$$

In the 10-1 and 10-2 $A^{2} I I-X^{2} \Sigma^{+}$Comet-Tail bands, Bulthuis [35] found a perturbation of the $\mathrm{R}_{12} \mathrm{Q}_{11}$ and $\mathrm{Q}_{12} \mathrm{P}_{11}$ branches. The perturbed level is $\mathrm{A}^{2} \Pi_{1 / 2}, v=10$. From a knowledge of the $v^{\prime}=9$ and 11 levels the unperturbed branches of $\mathrm{A}^{2} \mathrm{II}_{1 / 2}$ $\mathrm{X}^{2} \Sigma^{+}$for $v^{\prime}=10$ can be accurately calculated by addition of the mean values of $A^{2} \Pi_{1 / 2}-A^{2} \Pi_{3 / 2}$ differences of these two known levels to the measured branches of $\mathrm{A}^{2} \Pi_{3 / 2}-\mathrm{X}^{2} \Sigma^{+}\left(v^{\prime}=10\right)$. The $\mathrm{O}-\mathrm{C}$ (observed-calculated) differences for the lines are the energy shifts due to the perturbation. The shifts are small (about $3 \mathrm{~cm}^{-1}$ at most) (see fig. 3 of ref. 35 ) and include $J=3 \frac{1}{2}$ to $21 \frac{1}{2}$, the range observed. 'This can be understood from the results of Ittmann's [112] theory where the matrix elements determining the magnitude of the shifts are small for small $J$.

The perturbations occur for numerous $J$ values as can be seen from figure 3 of reference 35 . Here, for $v=10$, the crossing of perturbing levels occur at very low $J$ values. Hence, the perturbation is observed for many $J$ values because for small $J$ the two perturbing potential energy curves are more parallel than in the case of high $J$, since the energy differences for the same $\Delta J$ increases with J. 'This follows also because the interacting levels have very similar B values.

All perturbing energies are negative $(\mathrm{O}-\mathrm{C})$ as seen from figure 3 of reference 35 ; hence, the perturbing level lies entirely above the perturbed $\mathrm{A}^{2} \Pi_{1 / 2}$ level. By making use of Biskamp's [24] band head formula, the perturber is found to be $\mathrm{X}^{2} \Sigma^{+}, v=18$. Extrapolating from figure 3 of reference 35 , for $J=0$, the ratio of perturbations in the two $\Lambda$-components is 2.5:3.6. This ratio can be used to calculate the energies of the two perturbing ${ }^{2} \Pi-{ }^{2} \Sigma$ levels using Ittmann's [112] formulas. See figure 4 of reference 35 for energy curves of the perturbing levels.

The details of the perturbations are given in table $54 \mathrm{~g}$.

\section{Dissociation Energies, Predissociations, and Convergence Limits}

\subsection{Dissociation Energy of CO}

After a long history of controversy, the dissociation energy of carbon monoxide $\mathrm{D}^{0}(\mathrm{CO})$, having a value of $89460 \mathrm{~cm}^{-1}$ or $11.091 \mathrm{eV}$ [28], can now be regarded as generally accepted, though the triplet levels of the atomic dissociation products are not known. From the noncrossing rule the products would be $\mathrm{C}\left({ }^{3} \mathrm{P}_{0}\right)+\mathrm{O}\left({ }^{3} \mathrm{P}_{2}\right)$, but this has not been established as yet. Since the $\mathrm{X}^{1} \Sigma^{+}$state of $\mathrm{CO}$ dissociates to ground state products $[69,56]$ it is possible to make a unique correlation between a number of excited molecular states and their dissociation products (table 1 ).

Reviews concerned with the dissociation energy of $\mathrm{CO}$ have favored a variety of values. (Refs. $98,235,70,87,84,69,151,88,28,56$ and others.) The work of Gaydon [69], Douglas and Móller [56], Brewer et al. [27], and Brewer and Searcy [28] has resolved this question in favor of the "high" value, with several minor inconsistencies still to be ironed out.
In his review a decade ago, Gaydon [69] favored a value of $\mathrm{D}^{0}$ near $11.1 \mathrm{eV}$ based on application of the noncrossing rule and an exhaustive comparison of the evidence firom spectroscopy, electron impact, photoionization, and determinations of the heat of sublimation of carbon $\mathrm{L}(\mathrm{C})$. Since then, Douglas and Moller [56] have made high dispersion observations $(0.23$ to $2.5 \AA / \mathrm{mm})$ of the $\mathrm{B}^{1} \Sigma^{+}-\mathrm{A}^{1} \Pi$ Angstrom bands (including those of isotope $\mathrm{C}^{13} \mathrm{O}^{16}$ ) and $\mathrm{A}^{1} \Pi-\mathrm{X}^{1} \Sigma^{+}$Fourth Positive bands. They also reviewed the data on suspected predissociations of the $\mathrm{A}^{1} \Pi$ state concluding that none of these were real. This laid to rest predissociation data in support of values of $\mathrm{D}^{0}$ lower than $11.1 \mathrm{eV}$ and confirmed the ralue obtained from predissociations of the $\mathrm{B}^{1} \Sigma^{+}$and $b^{3} \Sigma^{+}$states. The results [56] also denied the existence of any appreciable maxima of the potentials in $\mathrm{CO}$, though Hagstrum [87] had clained one of about $0.2 \mathrm{eV}$ at $r>2.9 \AA$ for the $a^{\prime 3} \mathrm{~s}^{+}$state.

Brewer and Searcy [2S] list as best value for $\mathrm{D}^{\circ}(\mathrm{CO}) \quad S 9460 \pm 150 \quad \mathrm{~cm}^{-1}=11.091 \pm 0.019 \mathrm{er}$. 
The probable error is $\pm 92 \mathrm{~cm}^{-1}$. These uncertainties are based on the value $89595 \pm 30$ $\mathrm{cm}^{-1}$ obtained by Douglas and Møller [56] as an upper limit assuming the dissociation products are $\mathrm{C}\left({ }^{3} \mathrm{P}_{0}\right)+\mathrm{O}\left({ }^{3} \mathrm{P}_{2}\right)$. This was in close agreement with the value $89620 \pm 50 \mathrm{~cm}^{-1}$ obtained earlier [72,224] from a limiting curve of dissociation. The lower limit is $89325 \mathrm{~cm}^{-1}$ if the dissociation products are $\mathrm{C}\left({ }^{3} \mathrm{P}_{2}\right)+\mathrm{O}\left({ }^{3} \mathrm{P}_{0}\right)$. The mean of these extreme values is that given by Brewer and Searcy. $D^{\circ}$ is defined as the energy difference between the ground electronic state of the molecule and the energy of the atomic dissociation products in their ground states (e.g., $\left.\mathrm{C}\left({ }^{3} \mathrm{P}_{0}\right)+\mathrm{O}\left({ }^{3} \mathrm{P}_{2}\right)\right)$. Although it is most likely that the dissociation products are in their ground states, the mean value given by Brewer and Searcy is listed here as best value in order to underline the uncertainty. For thermodynamic calculations, however, ground state products are assumed to correlate with the observed predissociation. Thus Evans and Wagman [61] have used $89595 \mathrm{~cm}^{-1}$ for $\mathrm{D}^{\circ}(\mathrm{CO})$ to be consistent with previous usage.

Recent electron impact work [88, 62] and shock wave experiments [259,132] also support a value of $\mathrm{D}^{0}(\mathrm{CO})$ of about $11 \mathrm{eV}$.

The only spectroscopic evidence supporting a lower value of $\mathrm{D}^{0}$ is the fact of missing bands of $b^{3} \Sigma^{+}-a^{3} \Pi$ with $v^{\prime}=2$, discussed by Gaydon [69] and Barrow et al., [13] which suggests $\mathrm{D}^{0} \leq 10.94$ $\mathrm{eV}$. This missing structure is presently an anomaly which requires further consideration. The reported observation of the $2-0 \mathrm{~B}-\mathrm{X}$ band [255] which lies above the dissociation limit is somewhat uncertain, though the $v^{\prime}=2$ level could be populated by inverse predissociation. Only the early work of Ang'strom and Thalén (in 1875) [see footnote in ref. 23] mentions a band for $\mathrm{B}, v=2$; but this too is uncertain.

\subsection{Predissociations and Convergence Limits}

The most precise determination of $\mathrm{D}^{0}(\mathrm{CO})$ has been obtained from the data on predissociations of $\mathrm{B}^{1} \Sigma^{+}, v=0,1$ and $b^{3} \Sigma^{+}, v=0,1$ by the common state $a^{33} \Sigma^{+}\left(\right.$or $\left.f^{3} \Sigma^{+}\right)[72,56]$. In these instances, where the rotational structure is broken off for at least two successive vibrational levels [235], the actual dissociation limit lies close to the predissociation limit. Furthermore, the state causing the predissociations must be bound. Glockler [84] is therefore incorrect in his assertion that a repulsive state predissociates the $\mathrm{B}$ state.
Termination of vibrational structure in other states may indicate predissociation $[235,221]$ by a state or states as yet unspecified, and possibly repulsive. Such is the case for the states $\mathrm{C}^{1} \Sigma^{+}$, $c^{3} \Sigma^{+}, \mathrm{E}$, and $\mathrm{F}$, for which only $v=0$ or 1 is known.

A dissociation limit may be obtained from convergence of a state's vibrational structure or from a Birge-Sponer extrapolation (which should only be made with caution for excited states). In this regard, the $a^{\prime}$ and $\mathrm{F}$ states raise the most interesting questions (sec. 5.2e, f).

Discussion of the known and suspected predissociations, and convergence limits follows. A summary of the predissociations is given in table 55 .

\section{a. Predissociation of the $\mathrm{B}^{1} \Sigma^{+}$state}

Coster and Brons [43, 44] reported a sudden drop in intensity by a factor of two for the lines $\mathrm{P}(39), \mathrm{Q}(38)$ and $\mathrm{R}(37)$ of the $0-1 \mathrm{~B}^{1} \Sigma^{+}-\mathrm{A}^{1} \Pi$ Angstrom band. A similar intensity drop was observed by Read [209] in the $0-0$ B-X band. This effect was attributed to predissociation of $\mathrm{B}^{1} \Sigma^{+}, v=0, \mathrm{~J}=38$ by some triplet state whose dissociation limit was near the predissociation limit. A forbidden transition was indicated because the rotational structure was weakened, not terminated.

Hulthén [108] observed lines of the 1-1 B-A band up to $J^{\prime}=14$ in the $P$ branch and $J^{\prime}=17$ in the $\mathrm{Q}$ and $\mathrm{R}$ branches, but made no mention of intensity irregularities. Schmid and Gerö [224] reported a sudden intensity drop for $\mathrm{J}^{\prime} \geq 18$ in the 1-0 and 1-1 B-A bands, i.e., 1-0, $\mathrm{P}(18)>>\mathrm{P}(19)$; $\mathrm{Q}(17)>>\mathrm{Q}(18)]$. A dissociation limit of 89620 $\pm 47 \mathrm{~cm}^{-1}$ was obtained [74,226].

Douglas and Møller [56] reexamined the B-A Angstrom bands and confirmed these predissociations. In addition, they established these predissociations for $\mathrm{C}^{13} \mathrm{O}^{16}$ at $\mathrm{B}^{1} \Sigma^{+}, v=0$, between $\mathrm{J}=39$ and 40 , and $v=1$, between $\mathrm{J}=19$ and 20 . (A portion of the $0-2$ band of $\mathrm{C}^{13} \mathrm{O}^{16}$ showing the weakening of the lines is reproduced in fig. 1 of ref. 56.)

\section{b. Predissociation of the $b^{3} \Sigma^{+}$state}

A convergence limit for the perturbations of $b^{3} \Sigma^{+}, v=0$ is found for $55<N<56$, and considered by Dieke and Mauchly [54] to be a result of predissociation. It is not possible to detect the predissociation by a sudden intensity drop because 
the numerous perturbations already confuse the intensity distribution of the lines. Indirect evidence supporting the predissociation is that the analysis of Dieke and Mauchly [54] stops at $\mathrm{N}^{\prime}=55$. Of all the branches observed by them 23 of the strongest 36 break off just at $N^{\prime}=55$ : $\mathrm{Q}(55)$ twelve times, $\mathrm{P}(56)$ five times, and $\mathrm{R}(54)$ six times. No line was found for $\mathrm{N}^{\prime}>55$.

It is assumed that the cause of this predissociation as well as that of $\mathrm{B}^{1} \Sigma^{+}$is the same-the $f^{3} \Sigma^{+}$state (or $a^{\prime 3} \Sigma^{+}$state). (Ref. 72 , fig. 2 is obtained from the method of Buttenbänder and Herzberg.)

Brons [30] confirmed the predissociation of $b^{3} \Sigma, v=0, \mathrm{~J}=55$ in his observation of an intensity drop in the 0-1 Third Positive band. The suspected predissociation $[54,226]$ is found by Gerö [75] in the 1-0 $b^{3} \Sigma^{+}-a^{3} \Pi$ Third Positive band at $N \sim 43$. The branch lines $R_{1,2,3}(41), Q_{1,2,5}(42)$, and $P_{1,2,3}(43)$ all have regular intensity; the next lines are unobserved.

The 1-0 Third Positive band reported by Schmid and Gerö [226] at $2673 \AA$ [it should be $2665 \AA$ ] shows successive perturbations [clusters of lines looking like triplets] converging to a limit at $2628.3 \AA\left(38036.7 \mathrm{~cm}^{-1}\right)$, which seems to be the same predissociation limit as that for the B state in the Angstrom bands [72]. If it is assumed that the perturbations are caused by successively closer terms blending into a continuum, then a transition from this lower limit to the $a^{3} \Pi$ state corresponds to an energy of $38036.7 \mathrm{~cm}^{-1}(v=0)$ which corresponds to $\mathrm{Q}(43)$. (The limit at $38036.7 \mathrm{~cm}^{-1}$ corresponds to $\mathrm{Q}(\mathrm{N}=43)$. See also ref. 75.$)$

\section{c. Predissociation of the $\mathrm{C}^{1} \Sigma^{+}$state}

Schmid and Gerö [224b, 221] have interpreted as due to predissociation a sudden intensity drop at $\mathrm{J}=29$ in band lines of the $\mathrm{C}^{1} \Sigma^{+}-\mathrm{A}^{1} \Pi$ Herzberg bands originating from level $v^{\prime}=0$. This limit is about $3900 \mathrm{~cm}^{-1}$ or $0.48 \mathrm{eV}$ above the limit obtained from the $\mathrm{B}^{1} \Sigma^{+}$predissociation, and hence provides only an upper limit to the dissociation energy. The $\mathrm{C}^{1} \Sigma^{+}$state lies below $c^{3} \Sigma$; for neither state are there bands with $v^{\prime}>1$. 'This would appear to confirm the reality of the predissociation. (See ref. 235.) The C-X, 1-0 band has been observed in absorption [276] and shows no evidence of predissociation. However, predissociation is difficult to detect in absorption.
Gerö [74] included in his tabulation of suspected predissociations the breaking off in the $\mathrm{C}$ state of rotational structure between $93486-93608 \mathrm{~cm}^{-1}$ (average $93552 \mathrm{~cm}^{-1}$ ). What has proved to be an incorrect assignment of $\mathrm{C}\left({ }^{1} \mathrm{~S}\right)+\mathrm{O}\left({ }^{1} \mathrm{D}\right)$ at 933.35 $\mathrm{cm}^{-1}$ above $\mathrm{X}$ led Gerö to conclude that a repulsive state caused the predissocintion because of the $217 \mathrm{~cm}^{-1}$ difference between the atomic product and predissociation limits (i.e., kinetic energy of the dissociation products).

\section{d. Suspected Predissociation of the $c^{3} \Sigma^{+}$state}

Schmid [221] reported a possible predissociation of the $c^{3} \Sigma^{+}$state at $11.5 \mathrm{eV}$ because only the $v^{\prime}=0$ progression is known for the $c^{3} \Sigma^{+}-a^{3} \Pi 3 \mathrm{~A}$ bands, and the upper level lies close to the $\mathrm{C}^{1} \Sigma^{+}$, $v=0$ level which undergoes predissociation. A further reason for suspecting predissociation [77] is that the few observed bands of the $c^{3} \Sigma^{+}-a^{3} \Pi$ $3 \mathrm{~A}$ system are short and drop off in intensity rather abruptly about $10 \AA$ from the head, while similar exposures of other bands stretch for 50 to $100 \AA$. $\left(J_{\max }\right.$ in this case, assuming $\mathrm{T}=300$ ${ }^{\circ} \mathrm{K}$ and $10^{4}{ }^{\circ} \mathrm{K}$, gives $\sim 7$ and $\sim 13$, respectively.)

Because of overlapping of the $3 \mathrm{~A}$ and $\mathrm{A}^{1} \Pi-\mathrm{X}^{1} \Sigma$ Fourth Positive bands, it is not possible to determine the exact rotational quantum number of the intensity drop in any branch. However, from the predissociation limit of $\mathrm{C}^{1} \Sigma^{+}$at $93554 \mathrm{~cm}^{-1}$, the position of the $c^{3} \Sigma^{+}$state at $92076 \mathrm{~cm}^{-1}$, and $\mathrm{B}_{0}\left[a^{3} \Pi\right]=1.9563 \mathrm{~cm}^{-1}$, the first weakened level is found to be at $\mathrm{J} \sim 27$.

\section{e. Convergence Limit of the $a^{\prime 3} \Sigma^{+}$state}

The $a^{\prime 3} \Sigma^{+}$state is known up to $v=23$ from $a^{\prime}-\mathrm{X}$ bands [101]. Perturbations of $\mathrm{A}^{1} \Pi$ identify $a^{\prime}, v=10$ to 26 ; perturbations of $b^{3} \Sigma^{+}$ have been identified as due to $a^{\prime}, v=32$ to 41 [234a]. Some uncertainty exists $[69,99]$ in the identity of the higher lying lerels which are probably confused with terms of the $f^{3} \Sigma^{+}$state, about which little is known. Rotational constants for these levels go to zero at a term ralue of about $89600 \mathrm{~cm}^{-1}$ above X [234a], which colresponds to the known dissociation limit.

Gaydon and Penney [70] have applied the noncrossing rule to the $a^{\prime 3} \Sigma^{+}$state and coucluded that this state must go to ground state atoms. If not, then the unlikely possibility arises that two ${ }^{3} \mathbf{\Sigma}^{+}$ states dissociating to ground state atoms must. lie completely below the $a^{\prime}$ state (and have been 
unobserved to date). The regularity of the $a^{\prime}$ state's vibrational levels (up to $v=23$ ) does not indicate avoidance of crossing.

Two bands earlier believed to originate in high vibrational levels of the $a^{\prime 3} \Sigma$ state most probably originate from the otherwise unobserved $f^{3} \Sigma^{+}$ state [78]. Some interaction to avoid crossing by these two ${ }^{3} \Sigma^{+}$states appears unavoidable. Perturbations of the $b^{3} \Sigma^{+}$attributed to the $a^{\prime}$ and $f$ states may arise from their joint interaction (e.g., perturbation of three states).

\section{f. Convergence Limit of $F\left(1 \Sigma^{+}\right)$state}

The $\mathrm{F}\left({ }^{1} \Sigma^{+}\right)$state with $\omega_{e}=2112 \mathrm{~cm}^{-1}$ and extraordinarily large $\omega_{e} x_{e}=198 \mathrm{~cm}^{-1}$ probably has the flattest potential curve and smallest dissociation energy of all stable states of CO [224b, 105]. (But see the footnote to $\mathrm{F}$ state in table 1.) A linear extrapolation gives a dissociation energy of 0.5 to $0.6 \mathrm{eV}$. Gaydon cautions against BirgeSponer extrapolations for excited states, especially when some may have potential maxima. However, the rather large value of $\omega_{e} x_{e}$ for the $\mathrm{F}$ state implies rapid convergence of the vibrational levels.

If the dissociation products are known for $\mathrm{F}$, then $\mathrm{D}^{0}$ for the $\mathrm{X}$ state can be obtained without knowledge of predissociations. Schmid and Gerö [224b] correlated this dissociation limit at $\sim 12.9$ $\mathrm{eV}$ with $\mathrm{C}\left({ }^{1} \mathrm{~S}\right)+\mathrm{O}\left({ }^{1} \mathrm{D}\right)$, which led to a low value of $\mathrm{D}^{0}$.

The details of the $\mathrm{F}$ state have not been published, and its identity as ${ }^{1} \Pi$ is uncertain [69, 99, 147]. It was thought to be definitely singlet [224b] because of the high intensity of the $\mathrm{F} \leftarrow \mathrm{X}^{1} \Sigma$ transition so that it would dissociate into either two triplet or two singlet atoms. Howell [105] rules out the former [but doesn't say why] and offers most probably products as $\mathrm{C}\left({ }^{1} \mathrm{D}\right)+\mathrm{O}\left({ }^{1} \mathrm{D}\right)$. This would mean a dissociation energy of the $\mathrm{F}$ state of about $4.4 \mathrm{eV}$ which appears much too high. On this basis he obtained $\mathrm{D}^{0}(\mathrm{X})$ as $9.6 \mathrm{eV}$ from the energy difference $\left[\mathrm{C}\left({ }^{1} \mathrm{D}\right)+\mathrm{O}\left({ }^{1} \mathrm{D}\right)\right]-\left[\mathrm{C}\left({ }^{3} \mathrm{P}\right)+\mathrm{O}\left({ }^{3} \mathrm{P}\right)\right]=3.3 \mathrm{eV}$. However, using $\mathrm{D}^{0}$ as $11.09 \mathrm{eV}$ and the $\mathrm{F}$ state dissociation limit as $12.9 \mathrm{eV}$ it follows that the dissociation products are most likely $\mathrm{C}\left({ }^{3} \mathrm{P}\right)+\mathrm{O}\left({ }^{1} \mathrm{D}\right)$, indicating a triplet molecular state. This could imply a dissociation energy of the $\mathrm{F}$ state of about $0.9 \mathrm{eV}$. Lefebvre-Brion et al., [147] predict the $\mathrm{F}$ state to be ${ }^{1} \Sigma^{+}$.
The branches of a ${ }^{3} \Sigma^{-}-{ }^{1} \Sigma^{+}$transition are ${ }^{\mathrm{P}} \mathrm{P},{ }^{\mathrm{P}} \mathrm{Q},{ }^{\mathrm{R}} \mathrm{Q}$, and ${ }^{\mathrm{R}} \mathrm{R}$ which under low resolution may look like the $\mathrm{P}, \mathrm{Q}$, and $\mathrm{R}$ branches of a ${ }^{1} \Pi-{ }^{1} \Sigma$ transition. The intensity of the $\mathrm{F}-\mathrm{X}$ transition suggests that probably a singlet state mixes with the $\mathrm{F}$ state (if triplet) to make strong an otherwise forbidden transition. The identity of the $\mathrm{F}$ state remains uncertain.

\subsection{Dissociation Energy of $\mathrm{CO}^{+}$, Ionization Potentials and Rydberg Series}

The dissociation energies of the ground states of $\mathrm{CO}$ and $\mathrm{CO}^{+}$are related as follows:

$$
\mathrm{D}^{0}\left(\mathrm{CO}^{+}\right)=\mathrm{D}^{0}(\mathrm{CO})+\mathrm{IP}(\mathrm{C})-\mathrm{IP}(\mathrm{CO})
$$

Substitution of known values into the right hand side gives

$$
\begin{aligned}
& \mathrm{D}^{0}\left(\mathrm{CO}^{+}\right)=11.091+11.267-14.013 \\
& \mathrm{D}^{0}\left(\mathrm{CO}^{+}\right)=8.345 \mathrm{eV}
\end{aligned}
$$

$\mathrm{IP}(\mathrm{C})$ was taken from Moore [166]; IP(CO) was obtained from the Rydberg series limit of Takamine et al. [253], and $\mathrm{D}^{\circ}(\mathrm{CO})$ was taken from the predissociation limit in $\mathrm{B}^{1} \Sigma^{+}$of $\mathrm{CO}$ [2S]. (The conversion factor used is given in appendix B.) If the dissociation products of the ionic molecule are known, then the dissociation energies of the various states can be given unequivocally.

Gaydon [69] has given a succinct review of earlier estimates for the dissociation energies of the three known states of $\mathrm{CO}^{+}$, based mostly on Birge-Sponer extrapolations of the vibrational levels of these states and the noncrossing rule. These arguments will be mentioned briefly for the sake of completeness. For the ground state of $\mathrm{CO}^{+}$, a linear extrapolation by Biskamp [24] beyond $v=13$ gave $\mathrm{D} \sim 9.9 \mathrm{eV}$, which Gaydon and Penney [70] believed was not accurate because of its length. The vibrational levels of the A state extrapolate beyond $v=14$ to $\sim 9.2 \mathrm{eV}$ [70]. The levels of the $\mathrm{B}$ state beyond $v=10$ extrapolate to about $9.3 \mathrm{eV}$ above the $\mathrm{X}$ state $[24,70]$.

There is no direct evidence as to the + or symmetry of the $\mathrm{X}$ and $\mathrm{B}$ states [70]. By assuming that they are both $\Sigma^{+}$states $[99,69]$, it must necessarily be concluded that the $\mathrm{X}$ and $\mathrm{B}$ states have different dissociation limits to avoid crossing. The lowest dissociation products of $\mathrm{CO}^{+}$are 
$\mathrm{C}^{+}\left({ }^{2} \mathrm{P}\right)+\mathrm{O}\left({ }^{3} \mathrm{P}\right)$ which can form ${ }^{2} \Sigma^{+},{ }^{2} \Sigma^{-}(2),{ }^{2} \Pi(2)$ and other's. Both the $\mathrm{X}^{2} \Sigma^{+}$and $\Lambda^{2} \Pi_{i}$ states give ground state atomic products. The $\mathrm{B}^{2} \Sigma^{+}$state dissociates to $\mathrm{C}^{+}\left({ }^{2} \mathrm{P}\right)+\mathrm{O}\left({ }^{1} \mathrm{D}\right)$. Hence an unambiguous energy level diagram can be drawn for the observed states of $\mathrm{CO}^{+}$(ref. 69, pp. 186-7).

$\mathrm{D}_{\mathrm{x}}\left(\mathrm{CO}^{+}\right)=8.345 \mathrm{eV}, \mathrm{D}_{\mathrm{A}}=5.815 \mathrm{eV}, \mathrm{D}_{\mathrm{B}}=4.654$ $\mathrm{eV}$, using $\mathrm{O}\left({ }^{1} \mathrm{D}\right)-\mathrm{O}\left({ }^{3} \mathrm{P}\right)=15867.7 \mathrm{~cm}^{-1}=1.967 \mathrm{eV}$ [166]. These values are obtained from the ionization potentials of $\mathrm{CO}$ using data from Rydberg series $[253,254]$ and electronic transitions in $\mathrm{CO}^{+}$ [202b, 202a, 205]. The values given in section 2 are repeated for convenience: $14.013 \mathrm{eV}, 16.544$ $\mathrm{eV}, 19.671 \mathrm{eV}$.

There have been numerous other determinations of the ionization potentials of CO [129] mostly from electron impact and photoionization $[65$, 151, 267]. Wilkinson's review [275] compares the uncertainties in the three methods.

\subsection{Dissociation Energy of $\mathrm{CO}^{2+}$}

$\mathrm{CO}^{2+}$ has not been observed spectroscopically but has been observed in electron impact experiments [263,55] at $41.8 \mathrm{eV}$ and $45.9 \mathrm{eV}$ above the ground state of CO. Vaughen [263] has shown that the preferred dissociation products of $\mathrm{CO}^{2+}$ should be $\mathrm{C}^{2+}+\mathrm{O}$ corresponding to a bound state with dissociation energy of $4.8 \mathrm{eV}$. 'This value is close to that of the isolectronic molecule $\mathrm{BeO}$ (4.4 eV according to Gaydon [69]; Herzberg [99] gives $3.9 \mathrm{eV}$ ). Hurley and Maslen [111] have developed approximate expressions for the potential energy curve for the lowest bound state of $\mathrm{CO}^{2+}$ which use a scale factor $<1$ multiplying the curve for CO,X. Dorman and Morrison [55] have assumed slightly larger $r_{e}$ than for $\mathrm{CO}, \mathrm{X}$ and have sketched the curve schematically. No further information is available on $\mathrm{CO}^{2+}$.

\section{Vibration-Rotation Spectrum of CO}

'The vibration-rotation bands of $\mathrm{CO}$ lie at wavelengths shorter than $5 \mu$. Observed bands of the $\Delta v=1$ sequence include those with $v^{\prime} \leq 5$; the $\Delta v=2$ sequence includes those with $v^{\prime} \leq 7$. Overtone bands 3-0 and 4-0 have also been observed $[102,191]$. Table 56 lists the observed band origins. Rotational constants derived from infrared measurements, including some isotopic data, are included in table 36 .

Recently it has been proposed [203a] that calculated positions of lines of the 1-0 and 2-0 bands based on measurements by Rank et al. [197, 198, 126] and Plyler et al. [192-3, 190, 194], be adopted as secondary standard wavelengths in the infrared region. (The relative accuracy of the standards is $\pm 0.0003 \mathrm{~cm}^{-1}$; absolute accuracy is $\pm 0.002 \mathrm{~cm}^{-1}$ [203b, 196].) From these data, Rank et al. [197, 198] have derived molecular and rotational constants. Benedict [17] has recalculated slightly different values of molecular constants for the ground electronic state (table 1) which are consistent with both the infrared measurements and the data from electronic spectra. Analysis of new measurements on the fundamental band [17, 63 ] to $J$ values up to at least 70 will provide a good test of the uncertainty in these constants.

\section{1. $\Delta v=1$ Sequence}

The earliest observations of $\mathrm{CO}$ vibration-rota- tion bands were by Lowry [155] and Snow and Rideal [241]. Fine structure of the fundamental and overtone bands was first resolved by Whitcomb and Lagemann [272], and for the isotopic fundamental $\left(\mathrm{C}^{13} \mathrm{O}^{16}\right)$ by Lagemann et al., [139]. Mills and Thompson [162] have since measured the fundamental bands of $\mathrm{C}^{13} \mathrm{O}^{16}$ and $\mathrm{C}^{12} \mathrm{O}^{18}$ (with line positions good to $\pm 0.03 \mathrm{~cm}^{-1}$ ). Plyler et al. [193], in addition to $1-0$, measured some lines of the 2-1 band of these isotopes.

Benedict et al., [18] have measured the fundamental bands of the isotopic molecules $\mathrm{C}^{13} \mathrm{O}^{16}$, $\mathrm{C}^{13} \mathrm{O}^{18}$, and $\mathrm{C}^{12} \mathrm{O}^{18}$ relative to the $1-0$ band of $\mathrm{C}^{12} \mathrm{O}^{16}$, in addition to making nieasurements on the 2-1 band of $\mathrm{C}^{12} \mathrm{O}^{16}$. (Individual line measurements were reproducible to $\pm 0.02 \mathrm{~cm}^{-1}$; isotopic line positions relative to $\mathrm{C}^{12} \mathrm{O}^{16}$ are accurate to $\pm 0.01 \mathrm{~cm}^{-1}$ and are in close agreement with positions calculated from the molecular constants of Goldberg and Müller [85].)

Laser action on $\mathrm{P}$ branch lines in the $6-5,7-6$, $8-7,9-8$, and $10-9$ vibrational bands has been reported by Patel and Kerl [185]. (See also references listed therein.)

\subsection{Overtone Sequence and other Overtone Bands}

Plyler et al., [191] have observed lines of the $2-0,3-1,4-2$, and $5-3$ bands in the orertone 
region. Isotope shifts for the origin of the 2-0 bands were reported for $\mathrm{C}^{12} \mathrm{O}^{16}-\mathrm{C}^{13} \mathrm{O}^{16}$ and $\mathrm{C}^{12} \mathrm{O}^{16}$ $-\mathrm{C}^{12} \mathrm{O}^{18}$. Goldberg and Müller [85] identified nearly 300 lines of $\mathrm{CO}$ between $2.29-2.50 \mu$ in the spectrum of the solar limb to $\mathrm{J}$ values above 70 . The overtone sequence included $2-0$ to $7-5$, with measured wavelengths referred to the center of the solar disk. Derived molecular constants are in close agreement with the current best values. (See refs. 161, 165 for a listing of solar infrared CO lines.) Plyler et al., [190] have measured the same sequence. Transitions till 11-9 were seen, but overlapping allowed precision measurements only on 2-0,3-1, and 4-2 to $\mathrm{J}$ values near 70 . Recently, St. Pierre [273, 218] has remeasured lines of the $2-0,3-1$, and $4-2$ bands with improved precision. Rank [197] has determined the 3-0 band origin from interferometric measurement of the $R(6)$ line and use of precisely determined molecular constants.

Herzberg and Rao [102] have photographed the 4-0 band with wavelengths of unblended lines having relative accuracy of $\pm 0.01 \mathrm{~cm}^{-1}$, and absolute accuracy of $0.03 \mathrm{~cm}^{-1}$.

\section{Rotational Spectrum of CO (Microwave and Far Infrared)}

The pure rotational spectrum of $\mathrm{CO}$ has been studied in the microwave region (wavelengths $<2.6 \mathrm{~mm}$ ) and in the far infrared $(100-600 \mu)$. Observed transitions, rotational constants, electric dipole moment, $g$ factor, quadrupole moment $\mathrm{Q}$, and quadrupole coupling constant (eqQ) and other properties derived from the microwave spectra are summarized in tables 57-9. Calculated positions of the pure rotational lines of $\mathrm{C}^{12} \mathrm{O}^{16}$ have been reported by Rao et al., [204].

\subsection{Rotational Transitions in CO}

The first microwave study of $\mathrm{CO}$ was that of Gilliam et al., [83] who measured the $J=1 \leftarrow 0$ rotational transition of $\mathrm{C}^{12} \mathrm{O}^{16}$ and $\mathrm{C}^{13} \mathrm{O}^{16}$. Shortly thereafter, Bedard et al., [15] measured the $\mathrm{J}=1 \leftarrow 0$ and $J=2 \leftarrow 1$ transitions and obtained a value for $\mathrm{D}_{0}$ from their frequency separations. Cowan and Gordy [48] measured the $J=3 \leftarrow 2$ line in the $0.867 \mathrm{~mm}$ region. The first three rotational lines were seen together, and their separations caused by centrifugal stretching were measured directly giving a value of $\mathrm{D}_{0}$ close to that of Bedard et al., [15]. Gordy and Cowan [86] later measured the three lowest rotational transitions. Recently, Jones and Gordy [122] have measured the $4 \leftarrow 3$, $5 \leftarrow 4$, and $6 \leftarrow 5$ transitions in the submillimeter wave region. The observed rotational lines are listed in table 58 .

Rosenblum et al., [215] have made the best measurements of the $1 \leftarrow 0$ lines for a number of isotopes of carbon monoxide. The measurement of the magnetic moment [215] made possible three corrections to $\mathrm{B}_{e}$ for the moment of inertia of the electrons: (a) for the nonspherical electron dis- tribution, (b) wobble of the nuclei caused by rapid precession of electronic angular momentum in the molecule, and (c) the Dunham correction between $\mathrm{Y}_{01}$ and $\mathrm{B}_{e}$. (The magnetic moments and rotational constants are included in table 58.)

Palik and Rao [184] have observed the pure rotational absorption lines of $\mathrm{CO}$ for $\mathrm{J}=3$ to 23 in the 100 to $600 \mu$ region using a far infrared spectrometer with echelette gratings of 90 lines/in. and 45 lines/in. Positions of the lines from $J=0$ to 30 , calculated from the most precise infrared data $[197,85]$ have recently been proposed by Rao [203a, 204] as secondary standards in the far infrared.

\subsection{Stark Effect}

Burrus [36] has determined the electric dipole moment of CO to be $0.112 \pm 0.005$ Debye from the observed Stark shift of the $J=1 \leftarrow 0$ transition. Mizushima [163] has calculated a small correction for the polarizability Stark effect making the final value 0.114 Debye. (See table 57.) Rosenblum et al., [215] claimed to have determined the sign of the dipole moment from the relative magnetic moments as corresponding to the charge distribution $\mathrm{C}^{-} \mathrm{O}^{+}$. This is opposite in sign to that predicted from electronegativity differences alone. The molecular orbital calculations of Huo [109] and Nesbet [173] have shown that the assigned polarity $\mathrm{C}^{-} \mathrm{O}^{+}$is to be questioned. (See sec. 2.)

\subsection{Zeeman Effect}

Cox and Gordy [49] have observed the Zeeman effect in several linear and symmetric top mole- 
cules. For CO the rotational $g$ factor was found to be $0.268 \pm 0.005$ nuclear magnetons $\mu_{\mathrm{I}}$. In a linear molecule without nuclear coupling, the rotational magnetic moment is directed along the rotational momentum vector $\boldsymbol{J}$ but may point parallel or antiparallel as $g_{J}$ is + or - . Here only the magnitude of $g_{\mathbf{J}}$ was determined, but it is normally considered negative (except for hydrides). For a magnetic field of $10^{4} \mathrm{G}$ the $\sigma$ component separation is $4.08 \mathrm{MHz}$.

Rosenblum et al., [215] have determined the molecular magnetic moments for various isotopic species of carbon monoxide. For $\mathrm{C}^{12} \mathrm{O}^{16} g_{s}$ is $0.2691 \pm 0.0005 \mu_{\mathrm{I}}$. (See table 58.) Burrus [37] determined somewhat lower values from observation of the Zeeman splitting of the first rotational transitions. In a field of $10080 \mathrm{G}$, the separation was $4.115 \pm 0.025 \mathrm{MHz}$ for the $\sigma$ components of $J=1 \leftarrow 0$ and $4.095 \pm 0.050 \mathrm{MHz}$ for $J=2 \leftarrow 1$. Neither case showed $\pi$ splitting or

\section{Raman, Stark, and Zeeman Effects in Electronic Spectra of $\mathrm{CO}$ and $\mathrm{CO}^{+}$}

\subsection{Raman Effect}

Rasetti [206, 207] observed the fundamental vibrational frequency which was excited in $1 \mathrm{~atm}$ of $\mathrm{CO}$ by mercury radiation. Observation at 35 atm was made by Bhagavantam [20]. Cabannes and Rousset [38] observed the fundamental frequency and, in addition, determined the depolarization ratio $\rho$ to be 0.29 . Amaldi [1] reported the rotational Raman effect at 6 atm pressure, including the observation of 10 Stokes and 12 anti-Stokes lines.

\subsection{Stark Effect}

All experimental attempts to observe the Stark effect in the electronic spectra of $\mathrm{CO}$ and $\mathrm{CO}^{+}$ have yielded negative results because of insufficient resolution. Svensson [252] observed no splitting in the $\mathrm{B}-\mathrm{A}$ bands of $\mathrm{CO}$ in fields up to $115 \mathrm{kV} / \mathrm{cm}$ using a dispersion of $4 \AA / \mathrm{mm}$. Steubing [248] failed to observe splitting in the $\mathrm{CO}^{+} \mathrm{B}-\mathrm{X}$ bands with fields up to $67 \mathrm{kV} / \mathrm{cm}$, and dispersion of 5-9 $\AA / \mathrm{mm}$. Rave [208] observed no shift in fields up to $250 \mathrm{kV} / \mathrm{cm}$ for the $\mathrm{A}-\mathrm{X}$ bands $\left(\mathrm{CO}^{+}\right)$ with dispersion of $10 \AA / \mathrm{mm}$. From an assumed slit width of less than $0.02 \mathrm{~mm}$ and estimates of the resolving power of these authors, Kopelman broadening which indicates no change in $g$ factor with J. This appears to be the first check of $J$ independence of $g_{\mathrm{J}}$.

\subsection{Quadrupole Hyperfine Structure}

Rosenblum and Nethercot [214] have observed the hyperfine structure of the $J=1 \leftarrow 0$ transition of $\mathrm{C}^{12} \mathrm{O}^{17}$. The observed frequencies for the fully resolved $\Delta \mathrm{F}= \pm 1$ transitions are given in table 59. The value of (eqQ) is $+4.43 \pm 0.40$ $\mathrm{MHz}$. Q equal to $-0.0265 \pm 0.003$ barn is obtained from paramagnetic studies on $\mathrm{O}^{17}$ [123] confirming a microwave value of $0.026 \pm 0.009$ [249].

The rotational constants $\mathrm{B}_{0}, \mathrm{D}_{0}$, obtained from microwave measurements are given in table 58 .

The electric dipole moment and hyperfine structure obtained from microwave measurements are given in tables 57 and 59, respectively. and Klemperer [134] have estimated the dipole moments of several states (table 57). To observe the Stark splitting resolution of better than 0.5 $\mathrm{cm}^{-1}$ is necessary.

\subsection{Zeeman Effect}

The Zeeman effect, while important of itself, reveals in addition the existence of perturbations of rotational levels and the coupling case of electronic states. Zeeman patterns have been observed for three systems: $\mathrm{B}^{1} \Sigma^{+}-\mathrm{A}^{1} \Pi(\mathrm{CO})$, $b^{3} \Sigma^{+}-a^{3} \Pi \quad(\mathrm{CO})$, and $\mathrm{A}^{2} \Pi_{i}-\mathrm{X}^{2} \Sigma^{+}\left(\mathrm{CO}^{+}\right)$. A general review in 1934 by Crawford [51] on the Zeeman effect in diatomic spectra summarizes most of the work that has been done on CO.

Theory predicts the largest width patterns for low $\mathrm{J}$, with a maximum for $\mathrm{J}=\Lambda$. For large $\mathrm{J}$ values Zeeman displacements are generally immeasurably small, except in the nieghborhood of perturbations where the lines are very sensitive to the magnetic field. Such Zeeman patterns are large and irregular.

Kemble, Mulliken, and Crawford [12S] and Crawford [50] have observed symmetric patterns with displacements proportional to field strength (up to $35,000 \mathrm{G}$ ) for several bands of the $\mathrm{B}-\mathrm{A}$ system. Thirteen patterns were observed out of a 
possible 18 for the first two lines of the $P, Q$, and $R$ branches. (Greater intensity was observed for the low-frequency components of the $\mathrm{Q}$ doublets (l) polarization, $\Delta \mathrm{M}=0$ ) and of high-frequency components of $\mathrm{P}$ doublets $(\perp$ polarization, $\Delta \mathrm{M}=$ \pm 1 ) for high J.) Large displacements due to perturbations of the $A^{1} \Pi$ state were observed for $\mathbf{J}=23$ to 35 .

Watson [268, 269] has observed large and irregular Zeeman patterns for a number of magnetically sensitive lines in the $0-0$ and $1-0$ B-A bands using fields up to $30,000 \mathrm{G}$. Very violent perturbations were observed, expecially in the $0-0$ band. Numerous extra lines appeared. (See also ref. 216.) The $\mathrm{B}^{1} \Sigma^{+}$state, having no magnetic moment, is insensitive to the field.

Watson studied in detail the large displacements of lines for $\mathrm{J}>23$ previously observed by Crawford [50]. In the $0-0$ band, for $\mathrm{Q}(12)$, the displaced lines are strongly perturbed though fields of $10^{4} \mathrm{G}$ only increase their displacement (but do not produce a Zeeman pattern). A similar perturbation is observed in the $0-1$ band. The state perturbing $A^{1} \Pi$ is either case (a) $a^{3} \Pi$ [216] or $d^{3} \Delta_{i}$ (then thought to be ${ }^{3} \Pi_{r}$ ) in intermediate coupling between (a) and (b).

Schmid [236] has observed the Zeeman effect in the $b^{3} \Sigma^{+}-a^{3} \Pi$ bands in fields up to $29,000 \mathrm{G}$. Greater splitting or broadening was found near the heads than at higher $J . \quad R_{2}$ branch lines (with lower state ${ }^{3} \Pi_{1}$ ) are seen as sharp triplets with twice the normal component separation. $R_{1}$ and $R_{3}$ branch lines are weakened and broad. These effects confirmed the assignment of the $a^{3} \Pi$ state to case (b) by $\mathrm{N}=23$. Also, the $b^{3} \Sigma^{+}$state already displays a Paschen-Back effect for 15,000 G.

Schmid and Gerö [223] observed all lines split into symmetric doublets in the $\mathrm{A}^{2} \Pi_{i}-\mathrm{X}^{2} \Sigma^{+}$bands of $\mathrm{CO}^{+}$(fields 14,000 to $28,000 \mathrm{G}$ ). For $\Pi_{1 / 2}-\Sigma$, all doublets reached the maximum value $2 \Delta \sigma_{\text {normal }}$ for small J. The separation was smaller for large $J$ in this subband and for all $J$ in the $\Pi_{3 / 2}-\Sigma$ subband. Splitting was proportional to the field, and independent of vibrational quantum number. It is noteworthy that no deviation was detected to indicate perturbation of the $\left.\mathrm{A}^{2} \Pi_{1 / 2}\right) \quad v=5$ level (sec. 4.7).

\section{Molecular Energy Levels and Potential Energy Curves}

The potential energy curve for an electronic state of a diatomic molecule represents the sum of the coulomb repulsion of the nuclei and the electronic energy as a function of internuclear distance. This function (or curve) is defined when the electronic motion and nuclear motion do not interact directly, that is, away from the region of a perturbation or predissociation. Reliable potential energy curves can be calculated from the spectroscopically determined vibrational and rotational energy levels by the method of Rydberg-Klein-Rees (RKR). ${ }^{9}$

\footnotetext{
${ }_{9}^{9}$ The foundations of this method can be traced through reference 258 .
}

RKR curves for several states of CO have been calculated by Tobias et al. [258]. Krupenie and Weissman [138] have recalculated these using a numerical method [271] which avoids discontinuities in some integrals. Due account was taken of revised quantum numbering in some states, intermediate coupling, and better data in several instances. There is close agreement with previous calculations [258]. Potential curves for the three observed states of $\mathrm{CO}^{+}$are included for the first time.

Table 74 lists the term values, vibrational quanta, and turning points. The potential curves are displayed in figure 1. An energy level diagram is given in figure 2 . 


\section{Transition Probabilities and Lifetimes (Electronic Spectra)}

The integrated band intensity in emission is proportional to the relative vibrational transition probability or band strength which can be approximated by $p_{v^{\prime} v^{\prime \prime}}=\mathbf{R}_{e}^{2}(r) q$, where $\mathbf{R}_{e}$ is the electronic transition moment, and $q$ is the vibrational overlap integral square. The conditions under which this equation is valid have been discussed by Fraser [67]. It is often assumed that the electronic transition moment $\mathbf{R}_{e}$ is a slowly varying function of $r$ and that $\mathrm{R}_{e}$ can be replaced by an average $\mathbf{R}_{e}\left(\bar{r}_{v^{\prime} v^{\prime \prime}}\right)$, where the $r$ centroid is given by

$$
\bar{r}_{v^{\prime} v^{\prime \prime}}=\frac{\int \psi_{v^{\prime}} r \psi_{v^{\prime \prime}} d \tau}{\int \psi_{v^{\prime}} \psi_{v^{\prime \prime}} d \tau}
$$

When $R_{e}$ fluctuates considerably (perhaps even by a factor of 5) then the Franck-Condon factor $q$ becomes a poor approximation to the band strength, and the validity of the Born-Oppenheimer approximation breaks down.

The origin, properties, and methods of computation of $r$-centroids are discussed fully by Nicholls and Jarmain [180]. Detailed discussions of the concepts involved and the work done on numerous molecules can be found in a series of reviews [133, 242, 174, 175, 181, 178].

Evaluation of the Franck-Condon factor, the dominant term in the band strength, depends on the potential energy curves of the states involved. In virtually all cases (for $\mathrm{CO}$ and $\mathrm{CO}^{+}$) Morse functions have been assumed. Tables 60 to 72 summarize the numerical data on Franck-Condon factors, band strengths, $r$-centroids, and $I_{\infty}$ $\left(=\frac{p_{v^{\prime} v^{\prime \prime}}}{\lambda_{v^{\prime} v^{\prime \prime}}^{4}}\right)$ for a number of electronic transitions in $\mathrm{CO}$ and $\mathrm{CO}^{+}$. Lifetimes and oscillator strengths $f$ for several transitions have been measured (table 73). The experimental work and calculations are discussed below (including for some transitions, determination of the functional form of $\mathrm{R}_{e}$ ). The few quantitative measurements of band intensities are mentioned.

\section{1. $A^{1} \Pi-X^{1} \Sigma^{+}$Fourth Positive System}

Franck-Condon factors $q$ have been calculated by Nicholls [178] (table 60a), and $r$-centroids have been calculated by Jarmain et al., [113] (table 60b).
Other less extensive calculations of $q$ are in substantial agreement with the above values, except for some rough estimates [195] for high quantum numbers which differ by more than a factor of 10 . Nicholls [176] has shown from visual band intensity observations that $\mathrm{R}_{e}$ should be only weakly dependent on $r$ for a number of bands with $v^{\prime}=0$ to 4 , so that, for these, $p$ is well represented by $q$. Silverman and Lassettre [238] have reported good agreement between the experimental relative intensity for the $v^{\prime \prime}=0$ progression observed by alectron impact with those obtained from FranckCondon factors [113] corrected for band overlap. Lassettre [141] has indicated that more recent measurements with lower energy electron bombardment (and greater resolution) give poorer agreement.

\section{2. $\mathrm{B}^{1} \Sigma^{+}-\mathrm{A}^{1} \Pi$ Ångstrom System}

Ortenberg [183] and others [150, 14, 211] have calculated Franck-Condon factors (table 61) in substantial agreement with one another, by assuming Morse functions with $\omega_{e} x_{e}$ estimated $\left(23-27 \mathrm{~cm}^{-1}\right.$ ). These values (some with $v^{\prime}$ up to 11) are all rather uncertain because no level of the $\mathrm{B}$ state has been identified with certainty for $v>1$ because of predissociation. Robinson and Nicholls [211] have calculated $r$-centroids, $p$, and $I_{\infty}$ (table 61). Robinson [210] and Robinson and Nicholls [211] have measured relative intensities for the $0-0$ (8.2), $0-1(10), 0-2(3.2), 0-3(5.3)$ bands of the $v^{\prime}=0$ progression. $\mathrm{R}_{e}(r)$ is given const. $\left(0.768 r^{2}-1.745 r-1\right)$ for $1.12 \AA<r<1.19 \AA$ and is almost independent of $r$ in this range.

\section{$10.3 \mathrm{C}^{1} \Sigma^{+}-\mathrm{A}^{1} \Pi$ Herzberg System}

Leskov [150] has estimated $q$ for sereral bands (table 62) assuming a Morse function with $\omega_{c} x_{e}^{\prime}$ estimated as $18.07 \mathrm{~cm}^{-1}$. These values are uncertain since only the $v=0,1$ levels are known for $\mathrm{C}^{1} \Sigma^{+}$.

\section{$10.4 b^{3} \Sigma^{+}-a^{3} \Pi$ Third Positive System}

The absence of any recorded bands of the $b-a$ system for $v^{\prime}>2$ prompted Barrow et al., [13] to look into their intensity distribution. From assumed Morse functions, and the use of the 
Pekeris relation (ref. [99], p. 108) an estimate was made of $q$ relative to $q(0-0)$ and compared with their measured relative integrated band intensities. Comparison of intensities with $q$ showed that $\mathrm{R}_{e}$ varies significantly with $r$. Using a crude vibralional temperature and the ratio $\frac{q(2-0)}{q(0-0)}$, an estimate was made of the relative intensity of the $2-0$ band to be 0.023 times that of the $0-0$. A very long exposure at the expected wavelength of the 2-0 band showed no sign of a violet-degraded band, though some overlap by $\mathrm{A}-\mathrm{X}$ bands prevented a categorical denial of the existence of the $v^{\prime}=2$ level.

The 2-0 band is thus much weaker here than expected. If this be due to predissociation, then $\mathrm{D}^{\circ}(\mathrm{CO})<10.94 \mathrm{eV}$. If the perturbations of $\mathrm{b}, v^{\prime}=0,1$ extend to $v^{\prime}=2$ it is not clear how this could suppress the band completely [13]. It is suggested [13] that further study of $b$ levels be made from the $b-a$ and $b-\mathrm{X}$ systems in $\mathrm{C}^{12} \mathrm{O}^{16}$ and $\mathrm{C}^{13} \mathrm{O}^{16}$ to clarify this contradiction with the accepted dissociation energy.

Values of $q, r$-centroids, $p$, and $\mathrm{I}_{\infty}$ have been calculated [210, 211, 179, 256, 183] (table 63). $\mathbf{R}_{e}$ $(r)$ is given [256] as const. $(0.943 r-1)$ for $1.08 \AA<$ $r<1.16 \AA$, which confirms the large fluctuation observed by Barrow et al., [13]. In view of the crude approximations (estimates of $\omega_{e} x_{e}$ ) involved in determining the potential functions, it is suggested that the tabulated parameters be accepted with considerable reservation, since the $b$ state is predissociated. Schwenker [237a] has measured thelifetime of the $b-a$ transition and calculated the oscillator strength (table 73a).

\section{5. $a^{\prime 3} \Sigma^{+}-a^{3} \Pi$ Asundi System}

Jarmain et al. [114], have calculated FranckCondon factors assuming modified Morse functions (table 64).

\section{6. $a^{3} \Pi-X^{1} \Sigma^{+}$Cameron System}

Jarmain et al., [114] have calculated $q$ 's assuming modified Morse functions (table 65).

\section{7. $b^{3} \Sigma^{+}-X^{1} \Sigma^{+}$Hopfield-Birge System}

Nicholls [178] has calculated $q$ 's for the first two $v^{\prime}$ progressions (table 66).

\section{$10.8 a^{\prime 3} \Sigma^{+}-\mathrm{X}^{1} \Sigma^{+}$Hopfield-Birge System}

Nicholls [178] has calculated Franck-Condon factors for a large array of these transitions (table $67)$.

\section{9. $d^{3} \Delta_{1}-a^{3} \Pi$ Triplet System}

Pillow and Rowlatt [188] have calculated $\mathrm{I}_{\infty}$ $\left(=p_{0^{\prime} v^{\prime}} / \lambda^{4}\right)$ from the data of Herzberg [99] by using Pillow's distortion method and implicitly assuming that $\mathrm{R}_{e}$ is constant. Approximate mean wavelengths are quoted for the Triplet bands. Singh and Jain [239] have calculated $q$ and $\mathrm{I}_{\infty}$ from the same data as Pillow and Rowlatt, but assuming Morse functions. The two sets of values of $\mathrm{I}_{\infty}$ diverge from one another for $v^{\prime}>5$, with the Morse-based values preferred. Herman and Rakotoarijimy [93] have made measurements of the relative intensity of emission bands of the $v^{\prime \prime}=0$ progression observed in a high voltage discharge in $\mathrm{CO}$ diluted in xenon, and have calculated the $\mathrm{N}_{v}^{\prime}$ distribution. It is shown that, in this discharge, resonance excitation due to collision with metastable xenon atoms selectively populates the $v^{\prime}=6$ level. [In the above, corrected vibrational assignments have been ised.]

Singh and Jain [240] have calculated $q$ and $r$ centroids after having determined that the Morse

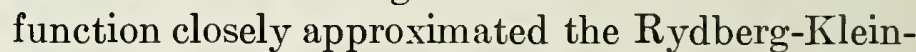
Rees (RKR) potential over a small range of $v$. (Their $q$ 's are essentially those of ref. 239.) Assuming $\mathrm{R}_{e}$ is approximately constant, they obtained $I_{\infty}$ from the Franck-Condon factors and estimates of $\mathrm{N}_{v}^{\prime}$ comparable to those of Herman [93]. All the above references use $v^{\prime}$ one unit lower than the revised numbering used here. This makes the numerical results somewhat uncertain. In any event, the assumption of constant $\mathrm{R}_{e}$ is untested. The values of $q, r$-centroids and $I_{\infty}$ are given in table 68 .

\subsection{0. $\mathrm{A}^{2} \Pi_{i}-\mathrm{X}^{2} \Sigma^{+}\left(\mathrm{CO}^{+}\right)$Comet Tail System}

Bennett and Dalby [19] have experimentally determined the oscillator strength $[f]$ for this transition, which was derived from the observed spontaneous emission lifetimes of several vibrational levels of the upper state. These quantities are given in table 73 . The lifetimes decrease slightly with increasing $v^{\prime}$, but are independent of $\mathrm{CO}$ pressure over the range $0.2 \mu$ to $6 \mu$. Constant $\mathrm{R}_{e}$ is assumed here. 
Arrays of $q, r$-centroids, $p$, and $\mathrm{I}_{\infty}$ have been calculated $[212,177,243,114,41]$ (table 69). Robinson and Nicholls [212] have ineasured relative band intensities photoelectrically. Absolute variation of $\mathrm{R}_{e}(r)$ is obtained [212, 177] as $43\left(-1+1.73 r-0.74 r^{2}\right)$ over the range 1.10 $\AA<r<1.21 \AA$ (a fluctuation of $20 \%$ ). The Einstein A coefficients, absolute band strengths, and band oscillator strengths were estimated (table 73) [177] for the A-X bands by combining the lifetime $\left(\tau_{v}\right)$ measurements of Bennett and Dalby [19] with the relative band intensities of Robinson and Nicholls [212] for a number of bands.

Spindler and Wentink [243] have obtained $\mathrm{R}_{e}=1.077 \pm 0.021$ Debye independent of $r$ between $1.074 \leq r \leq 1.184 \AA$ by using the lifetime measurements of Bennett and Dalby [19]. The disagreement between these two determinations of $\mathbf{R}_{e}$ is attributed [243] to possible systematic error in the intensity measurement by Robinson and Nicholls [212] due to regions of strong overlap. This discrepancy together with the nonconstant lifetimes $\tau_{v}^{\prime}$ from the $2-0$ and $2-1$, and also the 1-1 and 1-0 bands [19] makes desirable a recheck of the A-X Comet Tail system. (The calculated Einstein functions of ref. 243 differ by a factor of 2 from those of ref. 177.) The calculated absolption $f$ numbers disagree by a factor of three.

\subsection{1. $\mathrm{B}^{2} \Sigma^{+}-\mathrm{X}^{2} \Sigma^{+}\left(\mathrm{CO}^{+}\right)$First Negative System}

Nicholls [177] has calculated a large array of Franck-Condon factors and $r$-centroids (table 70 ). Lawrence [143] has measured lifetimes by means of the phase shift method and obtained Einstein coefficients and oscillator strengths for this system (table 73). Schwenker [237a], using a more conventional technique, has also measured the life- time of the $\mathrm{B}$ state which is about a factor of 2.5 smaller than that of Lawrence (table 73a). The discrepancy between these two values has not been explained.

\subsection{2. $\mathrm{B}^{2} \boldsymbol{\Sigma}^{+}-\mathrm{A}^{2} \Pi_{i}\left(\mathrm{CO}^{+}\right)$Baldet-Johnson System}

Nicholls [177] has calculated Franck-Condon factors and $r$-centroids (table 71 ).

\subsection{Ionization of $\mathrm{CO}$}

Wacks [264] has calculated Franck-Condon factors for ionizing transitions from $\mathrm{X}^{1} \Sigma^{+}(\mathrm{CO})$, $v=0$ to the $\mathrm{X}^{2} \Sigma^{+}, \mathrm{A}^{2} \Pi_{i}$, and $\mathrm{B}^{2} \Sigma^{+}$states of $\mathrm{CO}^{+}$ using Morse functions (table 72). In his consideration of ionization efficiency curves, it has been assumed that $\mathrm{R}_{e}(r)$ does not fluctuate. Halmann and Laulicht [88a] have since made a more extensive calculation for three isotopes of $\mathrm{CO}$ (in all cases assuming Morse functions).

\subsection{Miscellaneous}

Integrated oscillator strengths for the $\mathrm{A}-\mathrm{X}$, $\mathrm{B}-\mathrm{X}$, and $\mathrm{C}-\mathrm{X}$ transitions in $\mathrm{CO}$ have been obtained by Lassettre and Silverman [142] from inelastic electron scattering (table 73a). Absolute $f$-values for bands of the $\mathrm{A}-\mathrm{X}$ and $\mathrm{B}-\mathrm{X}$ systems have been determined by Hesser and Dressler [102a, b] from measured radiative lifetimes and previously calculated Franck-Condon factors [178] (tables $73 \mathrm{a}, \mathrm{c})$. 'The sums $\sum_{v^{\prime}} f_{v^{\prime} v^{\prime \prime}}$ confirm the magnitude of the integrated $f$-ralues [142].

Fox and Hickham [65] have measured the relative ionization probability to be $\frac{I(A)}{I(X)}=0.2$. For the $\mathrm{CO}$ continuum between $880 \AA-374 \AA$, the $f$-value is 2.8 [251].

\section{Summary and Conclusion}

The aims of inolecular spectroscopy include the determination of energy levels, molecular constants, potential energy curves, elucidation of the electronic structure, and the determination of transition probabilities. The status of our knowledge of these properties of $\mathrm{CO}, \mathrm{CO}^{+}$, and $\mathrm{CO}^{2+}$ will now be summarized.
Precise measurements were made years ago on bands of the $B-\Lambda$ system, and more recently on the $a^{\prime} \leftarrow \mathrm{X}, e^{\leftarrow}-\mathrm{X}, d \rightarrow a, \mathrm{E} \leftarrow \mathrm{X}$ systems and the three transitions of the ion. However, for several of these, only a few vibrational levels have been carefully studied. Work is in progress on analysis of high dispersion mensurements on the $\mathbf{A}-\mathbf{X}$, 
$e-\mathrm{X}, d-\mathrm{X}, \mathrm{I}-\mathrm{X}$, and $a^{\prime}-\mathrm{X}$ transitions $[100,238 \mathrm{a}]$. Definitive measurements have been made of the lowest pure rotational levels $\left(\mathrm{X}^{1} \Sigma^{+}, v=0\right)$ and for the lowest vibrational quanta (IR) in the electronic ground state. From these data, precise molecular constants have been determined which, together with the well-established dissociation energy, characterizes the $\mathrm{X}^{1} \Sigma^{+}$state as the best known for CO. However, even for this state, the vibrational levels (and potential energy curve) are known only halfway to the dissociation limit.

Much is known about the states lying below the first dissociation limit $(11 \mathrm{eV})$, the singular exceptions being the $f^{3} \Sigma^{+}$and $\mathrm{I}^{1} \Sigma^{-}$states. For most states lying above $11 \mathrm{eV}$, including all Rydberg states, the symmetry type, multiplicity, electronic structure, and dissociation products are unknown or uncertain. No Rydberg series have been observed in emission. Quantitative data are not available for any repulsive state.

Isotopes should be used to make unambiguous all vibrational quantum assignments. Critical re-examination of perturbations is needed, especially those pertaining to $A^{1} \Pi$ and $b^{3} \Sigma^{+}$, to unravel the overlapping structure of various states. Terms of the $a^{\prime 3} \Sigma^{+}$and $f^{3} \Sigma^{+}$states near the dissociation limit have not been separated. Franck-Condon factors based on Morse functions have been calculated for many transitions, but these values should be considered tentative. Quantitative intensity measurements are virtually nonexistent.

Two states of $\mathrm{CO}^{2+}$ have been observed only in electron impact experiment.

Note added in proof. Bands of the $\mathrm{T} \leftarrow \mathrm{X}$ and $\mathrm{S} \leftarrow \mathrm{X}$ Tanaka progressions have been reclassified as belonging to new B-X Rydberg series III and IV, respectively (Ogawa, M., Rydberg series converging to the $\mathrm{B}^{2} \Sigma^{+}$state of $\mathrm{CO}^{+}$, J. Chem. Phys. 43, 2142-4L (1965).

I am grateful to A. M. Bass for suggesting the review of the spectroscopic data on $\mathrm{CO}$ and for discussions with him which were useful in determining the scope and format of this review. Thanks are due also to C. Edmiston, T. Carrington, and M. Wacks for their helpful discussions. I wish further to acknowledge the effort of Mary Kirstein in carefully preparing the manuscript. 




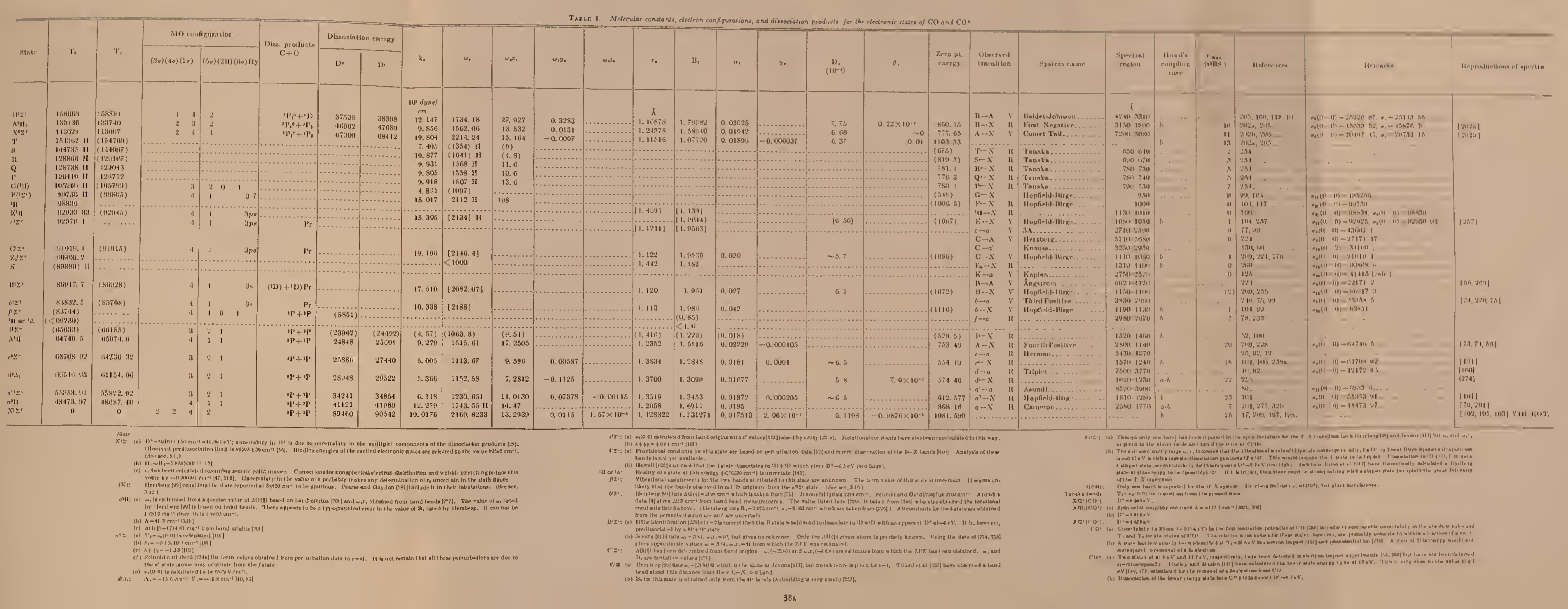




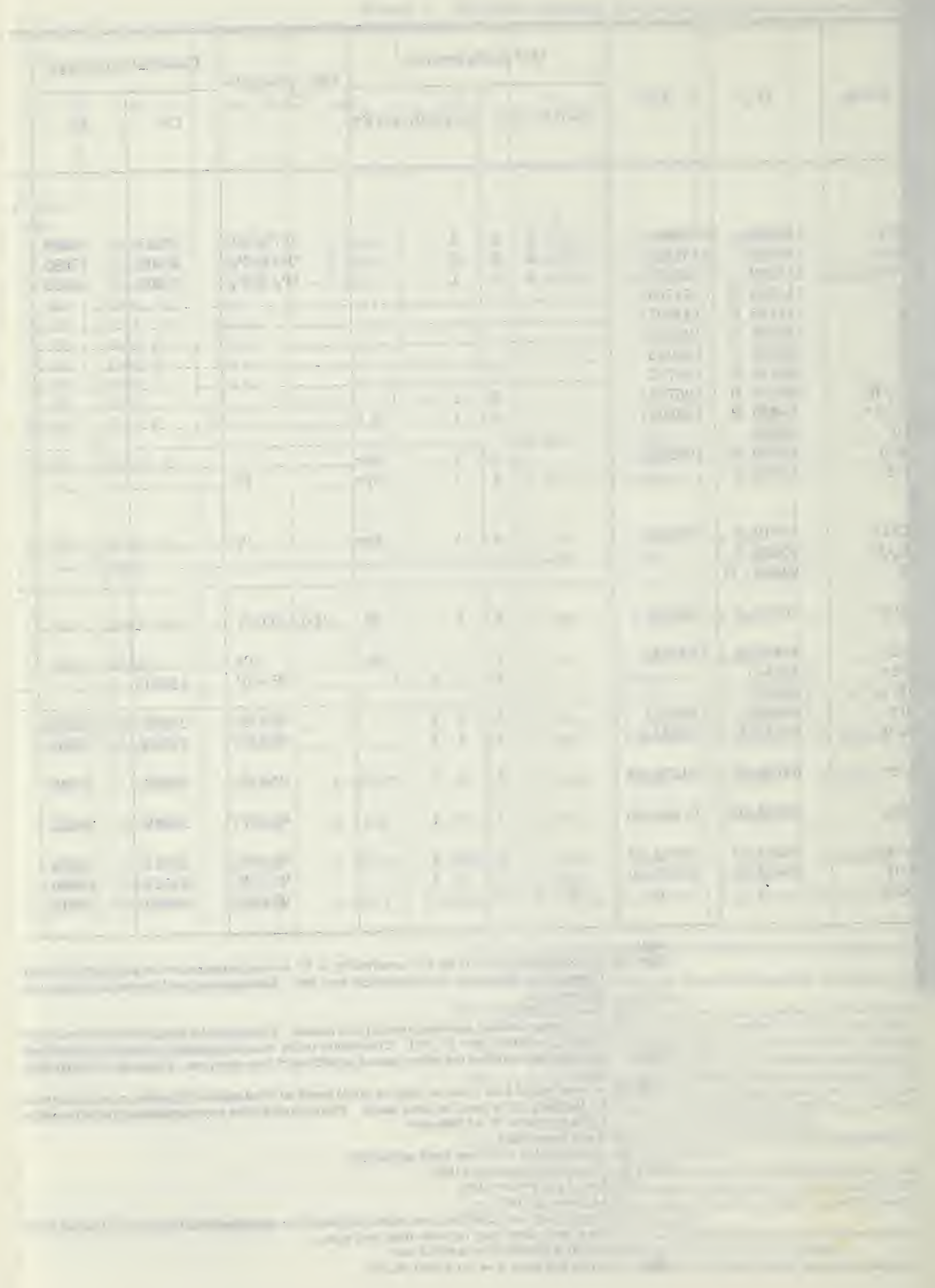


Table 1. Supplement. Rydberg stales

\begin{tabular}{|c|c|c|c|c|c|}
\hline Series & State $(n)$ & $T_{0}$ & $\omega_{0}$ & $\omega_{c} x_{0}$ & $\begin{array}{c}\text { Reproduction } \\
\text { of spectra }\end{array}$ \\
\hline \multirow[t]{4}{*}{$\mathrm{B}\left(\mathrm{CO}^{+}\right) \leftarrow \mathrm{X}$} & a 4 & 137760 & . & (-...- & $\boldsymbol{\beta}$ series [254] \\
\hline & 5 & 148712 & (1739) & $(22,8)$ & \\
\hline & 6 & 152812 & (1737) & $(20.3)$ & \\
\hline & 7 & 154820 & & & \\
\hline \multirow[t]{8}{*}{ Sharp } & 8 & 155943 & & & \\
\hline & 9 & 156637 & & & \\
\hline & 10 & 157082 & & & \\
\hline & 11 & 157416 & & & \\
\hline & 12 & 157627 & & & \\
\hline & Ref. [25 & ; (a) $\operatorname{Ref}$. & 07] & & \\
\hline & s 4 & 139530 & & & \\
\hline & 5 & 149105 & & & \\
\hline \multirow[t]{5}{*}{ Diffuse } & 6 & 153004 & & & \\
\hline & 7 & 154943 & & & \\
\hline & 8 & 156053 & & & \\
\hline & 9 & 156728 & & & \\
\hline & Ref. [25 & ; (a) Ref. & 07] & & \\
\hline \multirow[t]{6}{*}{$\mathrm{A}\left(\mathrm{CO}^{+}\right) \leftarrow \mathrm{X}$} & 5 & 123335 & & & \\
\hline & 6 & 127436 & & & \\
\hline & 7 & 129431 & & & \\
\hline & 8 & 130659 & & & \\
\hline & 9 & 131323 & & & \\
\hline & & f. [254] & & & \\
\hline \multirow[t]{10}{*}{$\mathrm{X}\left(\mathrm{CO}^{+}\right) \leftarrow \mathrm{X}$} & 6 & 106576 & {$[2185]$} & & \\
\hline & 7 & 108849 & [2171] & & \\
\hline & 8 & 110084 & [2181] & & \\
\hline & 9 & 110865 & {$[2161]$} & & \\
\hline & 10 & 111357 & [2181] & & \\
\hline & 11 & 111707 & {$[2175]$} & & \\
\hline & 12 & 111957 & [2172] & & \\
\hline & 13 & 112145 & {$[2167]$} & & \\
\hline & 14 & 112284 & {$[2167]$} & & \\
\hline & & f. [253] & & & \\
\hline
\end{tabular}

All above Rydberg series observed in absorption.
TABLE 2. Electron configurations for stales of $\mathrm{CO}$ and $\mathrm{CO}^{+\mathrm{c}}$

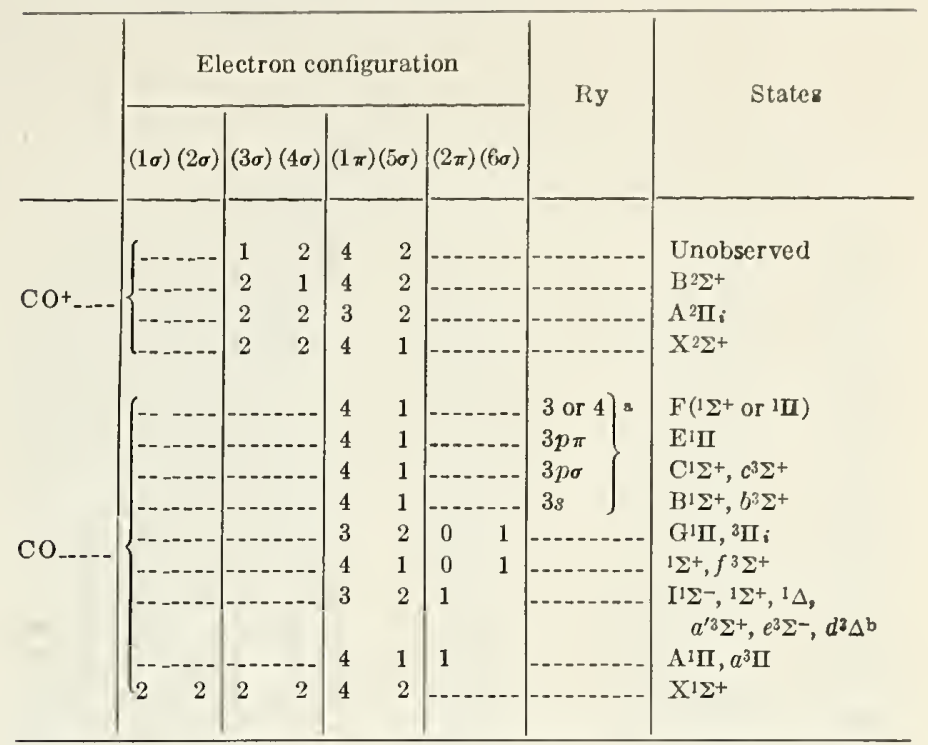

a These Rydberg states all converge to the ground state of $\mathrm{CO}+[147]$.

b The $d$ state is $d^{3} \Delta_{i}[40,137]$.

$\mathrm{C}\left({ }^{3} \mathrm{P}\right)+\mathrm{O}\left({ }^{3} \mathrm{P}\right)\left\{\begin{array}{l}\mathrm{X}^{1} \Sigma^{+}, 1 \Sigma^{+}, a^{3} \Sigma^{+}, f^{3} \Sigma^{+}, 8 \Sigma^{+}(2), \mathrm{I}^{1} \Sigma^{-}, e^{3} \Sigma^{-},{ }^{8} \Sigma^{-}, \\ \mathrm{A}^{1} \Pi,{ }^{1} \Pi{ }^{\mathrm{c}}, a^{3} \Pi,{ }^{3} \Pi,{ }^{5} \Pi(2),{ }^{1} \Delta^{\mathrm{c}}, d^{3} \Delta_{i},{ }^{3} \Delta\end{array}\right.$

c III or ${ }^{1} \Delta$ possibly lies below $66230 \mathrm{~cm}^{-1}$. 
TABLE 3. Band heads of the $\mathrm{A}^{1} \Pi-\mathrm{X}^{1} \Sigma^{+}$Fourth Positive system $(R)$

(a) Einission

\begin{tabular}{|c|c|c|c|c|c|c|c|c|c|c|c|c|c|c|}
\hline$\lambda$ & $\sigma$ & $I$ & $v^{\prime}-v^{\prime \prime}$ & Ref. & $\lambda$ & $\sigma$ & $I$ & $v^{\prime}-v^{\prime \prime}$ & Ref. & $\lambda$ & $\sigma$ & $I$ & $v^{\prime}-v^{\prime \prime}$ & Ref. \\
\hline$\stackrel{\circ}{A}$ & $c m^{-1}$ & & & & $\AA$ & $c m^{-1}$ & & & & $\stackrel{\circ}{A}$ & $c m^{-1}$ & & & \\
\hline 2799.7 & 35708.2 & 9 & $9-22$ & & 2137.0 & 46779.9 & 5 & $7-14$ & & 1603.3 & $(62371.4)$ & 1 & $9-7$ & $\mathrm{~L}$ \\
\hline 2785.4 & 35890.3 & 8 & $4-18$ & & 2128.3 & 46971.1 & 8 & $3-11$ & & 1597.14 & 62611.8 & 8 & $0-1$ & \\
\hline 2742.6 & 36450.7 & 6 & $11-23$ & & 2113.07 & 47309.51 & 9 & 613 & 182 & 1595.60 & 62672.4 & 2 & $6-5$ & \\
\hline 2740.0 & 36486.0 & 4 & $7-20$ & & 2107.2 & 47440.3 & 7 & $2-10$ & & 1576.67 & 63425.0 & 8 & $2-2$ & \\
\hline 2712.1 & 36860.5 & 4 & 6-19 & & 2089.9 & 47834.0 & 10 & 5-12 & & 1560.14 & 64096.9 & 7 & ${ }^{\mathrm{a}} 1-1$ & \\
\hline 2698. 30 & 37049.38 & 6 & $13-24$ & 74 & 2086.9 & 47901.5 & 1 & $1-9$ & & 1559.47 & 64124.4 & 8 & $4-3$ & \\
\hline 2684.0 & 37247.4 & 3 & 5-18 & & 2067.71 & 48347.17 & 10 & $4-11$ & 73 & 1545.3 & 64712.8 & 4 & b 6-4 & 90 \\
\hline 2680.8 & 37291.5 & 5 & $9-21$ & & 2046.3 & 48852.9 & 10 & $3-10$ & & 1544.2 & 64758 & $\ldots$ & ${ }^{c} 0-0$ & 255 \\
\hline 2662.9 & 37541.8 & 4 & $12-23$ & & 2034.35 & 49140.0 & 4 & 6-12 & 90 & 1542.34 & 64836.4 & 8 & $3-2$ & \\
\hline 2661.5 & 37561. i & 4 & $15-25$ & & 2031.7 & $(49204 . \quad)$ & 1 & $9-14$ & L & 1534.2 & $(65180)$. & 2 & $8-5$ & L \\
\hline 2659.6 & 37587.9 & 4 & $4-17$ & & 2025.8 & 49347.8 & 9 & $2-9$ & & 1527.52 & 65465.5 & 3 & $5-3$ & \\
\hline 2630.0 & 38011.4 & 6 & $11-22$ & & 2011.8 & 49690.6 & 8 & $5-11$ & & 1525.75 & 65541.6 & 6 & $2-1$ & \\
\hline 2598.31 & 38475.05 & 4 & $10-21$ & 73 & 2005.8 & 49839.8 & 5 & $1-8$ & & 1520.4 & (65772. ) & 1 & $12-7$ & $\mathrm{~L}$ \\
\hline 2594.5 & 38531.5 & 1 & $16-25$ & & 1990.89 & 50228.8 & 2 & 4-10 & & 1515. 7 & 65977.9 & 3 & $7 \cdot 4$ & 90 \\
\hline 2567.8 & 38932.8 & 5 & $9-20$ & & 1970.08 & 50759.3 & 3 & $3-9$ & & 1510.4 & 66206.3 & 2 & $4-2$ & 90 \\
\hline 2556.03 & 39111. 51 & 3 & $12-22$ & 74 & 1950.07 & 51280.2 & 3 & $2-8$ & & 1509.600 & 66240.0 & 6 & $1-0$ & \\
\hline 2538.6 & 39379.7 & 4 & $8-19$ & & 1939.12 & 51569.9 & 1 & $5-10$ & & 1506.8 & $(66366)$. & 2 & $9-5$ & $\mathrm{~L}$ \\
\hline 2524.1 & $(39606)$. & 4 & $11-21$ & $\mathrm{DE}$ & 1930.70 & 51794.8 & 3 & $1-7$ & & 1497.8 & $(66764)$. & 3 & $6-3$ & L \\
\hline 2521.8 & 39642.7 & 3 & $14-23$ & & 1918.08 & 52135.4 & 2 & $4-9$ & & 1493.60 & 66952.3 & 6 & $3-1$ & \\
\hline 2509.9 & 39830.1 & 8 & $7-18$ & & 1912.8 & 52279.9 & 1 & $0-6$ & 90 & 1488.0 & (fi7204. ) & 2 & $8-4$ & L \\
\hline 2492.86 & 40102.42 & 8 & $10-20$ & 73 & 1897.83 & 52691.6 & 4 & $3-8$ & & 1480.2 & 67556.6 & 2 & $5-2$ & 90 \\
\hline 2483.8 & 40248.8 & 3 & $6-17$ & & 1891.2 & $(52876.5)$ & 6 & $6-10$ & L & 1477.48 & 67682.9 & 3 & $2-0$ & \\
\hline 2463. 22 & 40585.12 & 10 & $9-19$ & 73 & 1878.33 & 53238.9 & 7 & $2-7$ & & 1475.4 & $(67778)$. & 1 & $12-6$ & L \\
\hline 2458.0 & 40671.5 & 2 & $5-16$ & & 1870.3 & $(53467.4)$ & 3 & $5-9$ & L & 1473.0 & $(67889)$. & 1 & $14-7$ & $\mathrm{~L}$ \\
\hline 2433.9 & 41057.5 & 9 & $8-18$ & & 1859.41 & 53780.4 & 5 & $1-6$ & & 1463.4 & 68333.3 & 10 & $4-1$ & 90 \\
\hline 2424.20 & 41238.16 & 5 & $11-20$ & 74 & 1850.1 & 54050.8 & 1 & $4-8$ & 90 & 1452.17 & 68862.5 & 5 & $6-2$ & \\
\hline 2407.6 & 41552.0 & 7 & $7-17$ & & 1846.7 & $(54150.6)$ & 2 & $7-10$ & L & 1447.27 & 69095.4 & 1 & 3-0 & \\
\hline 2394.2 & 41754.2 & 3 & $10-19$ & & 1841.47 & 54304.6 & 1 & $0-5$ & & 1443.7 & $(69266)$. & 1 & $8-3$ & L \\
\hline 2393.1 & 41773.9 & 4 & $13-21$ & & 1829.81 & 54650.6 & 4 & $3-7$ & & 1435.28 & 69672.9 & 7 & $5-1$ & \\
\hline 2381.6 & 41975.3 & 6 & $6-16$ & & 1825.38 & 54783.1 & 1 & $6-9$ & & 1425.78 & 70137.2 & 5 & $7-2$ & \\
\hline 2365.47 & 42261.95 & 5 & $9-18$ & 74 & 1810.82 & 55223.8 & 8 & $2-6$ & & 1418.91 & 7047 วิ. 6 & 1 & $4-0$ & \\
\hline 2356.5 & 42422.3 & 4 & $5-15$ & & 1804.71 & 55409.8 & 2 & $5-8$ & & 1414.0 & (70721. ) & 1 & $11-4$ & L \\
\hline 2337.95 & 42759.48 & 7 & $8-17$ & 73 & 1792. 38 & 55791.7 & 8 & $1-5$ & & 1411.4 & $(70852)$. & 1 & $13-5$ & L \\
\hline 2332.5 & 42860.0 & 3 & 4-14 & & 1784.9 & 56026.8 & 1 & $4-7$ & 90 & 1408.86 & 70979.6 & 5 & $6-1$ & \\
\hline 2311.47 & 43249. 27 & 8 & $7-16$ & 73 & 1774.90 & 56341.3 & 3 & $0-4$ & & 1401.02 & 71376.5 & 3 & $8-2$ & \\
\hline 2309.7 & $(43282 . \quad)$ & 4 & 3-13 & $\mathrm{DE}$ & 1747.20 & 57234.4 & 4 & $2-5$ & & 1395.7 & $(71$ t่49. $)$ & 2 & $10-3$ & L \\
\hline 2301.7 & $(43433 . \quad)$ & 2 & $10-18$ & $\mathrm{DE}$ & 1743.12 & 57368.4 & 1 & $5-7$ & & 1391.07 & 71813.8 & 1 & $5-0$ & \\
\hline 2286.1 & 43728.5 & 7 & $6-15$ & & 1729. 25 & 57828.7 & 7 & $1-4$ & & $13 \varsigma 4.00$ & 72254.2 & 3 & $7-1$ & \\
\hline 2273.9 & 43963.4 & 3 & $9-17$ & & 1723.79 & 58011.9 & 2 & $4-6$ & & 1377.75 & 72582.3 & 2 & $9-2$ & \\
\hline 2272.3 & 43994.0 & 1 & 12-19 & & 1712.19 & 58404.7 & 4 & $0-3$ & & 1373.7 & 72796.1 & 1 & $11-3$ & 40 \\
\hline 2261.7 & 44200.0 & 9 & $5-14$ & & 1705.16 & 58645.7 & 3 & $3-5$ & & 1371.8 & $(72897)$. & 2 & $i \cdot 3-4$ & $L$ \\
\hline 2247.2 & 44486.6 & 7 & $8-16$ & & 1704.30 & 58675.2 & 2 & $6 \cdot 7$ & & 1368.0 & $(73099)$. & 1 & $6-0$ & L \\
\hline 2238.3 & 44663.8 & 9 & $4-13$ & & 1684.9 & 59350.0 & 1 & $5-6$ & 90 & 1361.3 & $(73459)$. & 2 & $8-1$ & L \\
\hline 2221.5 & 45001.4 & 10 & $7-15$ & & 1669.68 & 59891.8 & 4 & $1-3$ & & 1355.84 & 73755.0 & 1 & $10-2$ & \\
\hline 2215.8 & 45115.9 & 3 & $3-12$ & & 1653.02 & 60495.5 & 7 & $0-2$ & & 1353.6 & $(73877)$. & 1 & $12-3$ & L \\
\hline 2196.8 & 45506.4 & 10 & $6-14$ & & 1647.90 & 60683.4 & 4 & $3-4$ & & 1339.0 & $(74683)$. & 1 & $0-1$ & $\mathrm{~L}$ \\
\hline 2194.0 & $(45565 . \quad)$ & 6 & $2-11$ & $\mathrm{DE}$ & 1630.40 & 61334.6 & 8 & $2-3$ & & 1335.0 & (74906. $)$ & 1 & $11-2$ & L \\
\hline 2188.1 & $(45687 . \quad)$ & 2 & $9-16$ & $\mathrm{DE}$ & 1629.61 & 61364.4 & 8 & $5-5$ & & 1316.0 & 75986.7 & 1 & $12-2$ & 90 \\
\hline 2173.01 & 46004.67 & 9 & $5-13$ & 73 & 1623.4 & $(61599.1)$ & 1 & $10-8$ & $\mathrm{~L}$ & 1299. 3 & 76965.7 & 1 & $11-1$ & 90 \\
\hline 2161.6 & $(46248 . \quad)$ & 8 & $8-15$ & $\mathrm{DE}$ & 1615.1 & $(61915.7)$ & 2 & $7-6$ & $\mathrm{~L}$ & 1280.5 & 78091.4 & 1 & $12-1$ & 90 \\
\hline 2150.2 & 46492. 6 & 8 & $4-12$ & & 1611,26 & 62063.3 & 3 & $4-4$ & & & & & & \\
\hline
\end{tabular}

Air wavelengths above $2000 \AA$ mostly from Estey [60]; vacuum wavelengths below $2000 \AA$ mostly from Read [209]. Inaccurate head measurements by Deslandres $=D E$, and Lyman $=\mathrm{L}$ are taken from ref. 23 and indicated here by parentheses ( ). Original measurements of Read [209] and Headrick and Fox [90] were all in vacuum. a Position calculated by Read [209], head obscured.

b Quantum assignment by Read [209]; band observed by Headrick and Fox [90].

- Absorption [255]. 
(b). Absorption

\begin{tabular}{|c|c|c|c|c|c|c|}
\hline \multirow[t]{2}{*}{$0^{\prime}$} & $v^{\prime \prime}=0$ & \multirow[t]{2}{*}{$\sigma$} & \multirow[t]{2}{*}{$I$} & $v^{\prime \prime}=1$ & \multirow[t]{2}{*}{$\sigma$} & \multirow[t]{2}{*}{$I$} \\
\hline & $\lambda$ & & & $\lambda$ & & \\
\hline & $\AA$ & $c m-1$ & & A & $c m-1$ & \\
\hline 0 & 1544.2 & 64758 & 6 & 1597.3 & 62606 & 3 \\
\hline 1 & 1509.8 & $\begin{array}{ll}66 & 234\end{array}$ & 7 & 1560.1 & 64098 & 2 \\
\hline 2 & 1477.5 & 67682 & 7 & 1526.8 & 65496 & 3 \\
\hline 3 & 1447.4 & $\begin{array}{ll}69 & 089\end{array}$ & 8 & 1493.9 & 66393 & 4 \\
\hline 4 & 1419.1 & $\begin{array}{lll}70 & 467\end{array}$ & 10 & 1463.5 & 68329 & 7 \\
\hline 5 & 1392.6 & $\begin{array}{ll}71 & 808\end{array}$ & 8 & 1435.3 & 69672 & 10 \\
\hline 6 & 1367.7 & $73 \quad 115$ & 7 & 1409.1 & 70967 & 9 \\
\hline 7 & 1344.1 & 74399 & 5 & 1384.2 & 72244 & 9 \\
\hline 8 & 1322.1 & $75 \quad 637$ & 3 & 1360.8 & 73486 & 6 \\
\hline 9 & 1301.4 & $76 \quad 840$ & 2 & 1338.8 & 74694 & 5 \\
\hline 10 & 1282.0 & 78003 & 1 & 1317.8 & 75884 & 3 \\
\hline 11 & 1263.6 & $79 \quad 139$ & 1 & 1298.6 & 77006 & 2 \\
\hline 12 & 1246.2 & $80 \quad 244$ & 0.7 & 1280.1 & $78 \quad 125$ & 1 \\
\hline 13 & 1229.8 & 81314 & 0.4 & 1263.4 & 79151 & 1 \\
\hline 14 & 1214.3 & $82 \quad 352$ & 0.3 & & & \\
\hline 15 & 1199.8 & $83 \quad 347$ & 0.1 & & & \\
\hline 16 & 1186.1 & 84310 & 0.07 & & & \\
\hline 17 & 1173.2 & $\begin{array}{ll}85 & 237\end{array}$ & 0.05 & & & \\
\hline 18 & 1161.2 & $86 \quad 118$ & 0.03 & & & \\
\hline 19 & . & . & $\ldots$ & & & \\
\hline 20 & 1139.7 & $87 \quad 742$ & 0.02 & & & \\
\hline
\end{tabular}

Data taken from Tanaka, Jursa, and LeBlane [255]. (Birge, Nature 124, 182-3 (1929) referred to measurement of the 0-0 to 15-0 transitions in absorption by Hopfield and Birge; details were never published.) 
TABLE 4. Deslandres table for the band origins of the $\mathrm{A}^{1} \Pi-\mathrm{X}^{1} \Sigma^{+}$Fourth Positive system

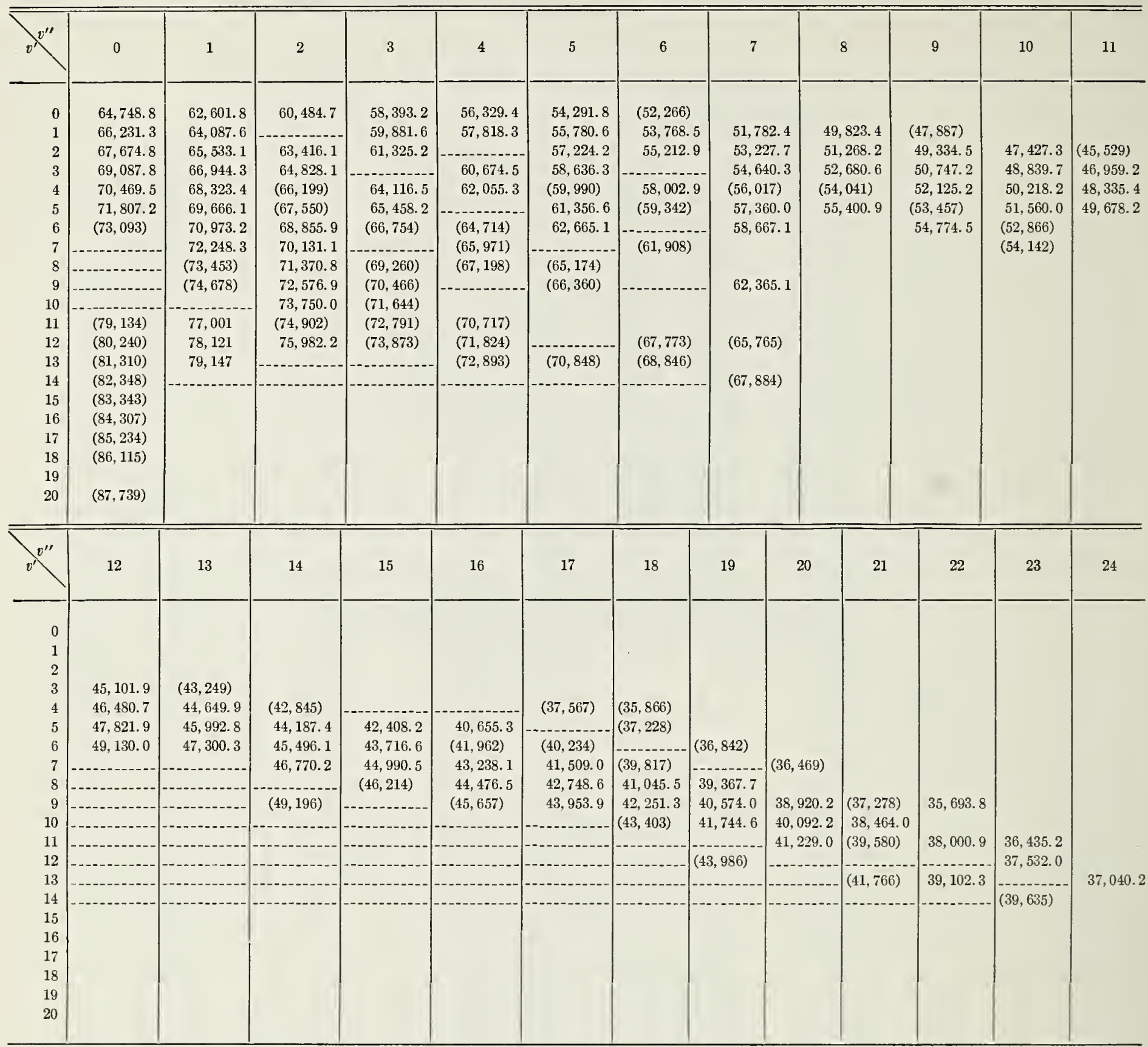

This table is essentially that given by Herzberg [99] with the additions he mentioned which have large quantum numbers. This is supplemented by origins at low quantum numbers based mostly on data of Tanaka, Jursa, and LeBlanc [255].

( ) Signifies data of much lower accuracy than the rest. 
TABLE 5. Band heads and origins of the $\mathrm{A}^{1} \mathrm{II}-\mathrm{X}^{1} \Sigma^{+}$ Fourth Positive system of $\mathrm{C}^{13} \mathrm{O}^{16}(R)$

(a) Emission

\begin{tabular}{|c|c|c|c|}
\hline$\lambda_{H}$ & $\sigma_{H}$ & $\sigma_{0}$ & $v^{\prime}-v^{\prime \prime}$ \\
\hline$\AA$ & $c m^{-1}$ & $\mathrm{~cm}^{-1}$ & \\
\hline 2613.8 & 38247.3 & 38234.1 & $8-20$ \\
\hline 2564.5 & 38982 & 38972 & $10-21$ \\
\hline 2559.5 & 39058.8 & 39043.7 & $6-18$ \\
\hline 2535.3 & 39431.4 & 39420.0 & $9-20$ \\
\hline 2507.2 & 39873.4 & 39861.3 & $8-19$ \\
\hline 2463.2 & 40586 & 40576 & $10-20$ \\
\hline 2454.2 & 40733.4 & 40719.7 & $6-17$ \\
\hline 2429.5 & 41148.0 & 41133.4 & $5-16$ \\
\hline 2405.7 & 41555.9 & 41540.4 & $4-15$ \\
\hline 2368.3 & 42211 & 42202 & $10-19$ \\
\hline 2356.0 & 42431.3 & 42418.7 & $6-16$ \\
\hline 2340.8 & 42707 & 42697 & 9-18 \\
\hline 2331.9 & 42870.2 & 42856.8 & $5-15$ \\
\hline 2314.3 & 43197 & 43187 & $8-17$ \\
\hline 2308.6 & 43302.2 & 43288.1 & $4-14$ \\
\hline 2264.1 & 44153.4 & 44141.8 & 6-15 \\
\hline 2240.6 & 44616.7 & 44604.4 & $5-14$ \\
\hline 2226.7 & 44896.2 & 44886.5 & $8-16$ \\
\hline 2217.9 & 45073.2 & 45060.2 & $4-13$ \\
\hline 2201.9 & 45402 & 45391 & $7-15$ \\
\hline 2178.0 & 45900.0 & 45889.2 & $6-14$ \\
\hline 2155.0 & 46388.2 & 46376.8 & $5-13$ \\
\hline 2133.0 & 46868.6 & 46856.5 & 4-12 \\
\hline 2120.3 & 47148.9 & 47139.2 & $7-14$ \\
\hline 2111.7 & 47340.4 & 47327.7 & $3-11$ \\
\hline 2097.0 & 47672.3 & 47662.1 & $6-13$ \\
\hline 2091.3 & 47803 & 47790 & $2-10$ \\
\hline 2074.7 & 48184.2 & 48173.5 & $5-12$ \\
\hline 2053.2 & 48689.5 & 48678.3 & 4-11 \\
\hline 2032.4 & 49186.4 & 49174.5 & $3-10$ \\
\hline 2020.8 & 49469 & 49459 & 6-12 \\
\hline 2012.5 & 49674.5 & 49662.1 & $2-9$ \\
\hline 1999.8 & 50005.2 & 49995. 1 & 5-11 \\
\hline 1978.8 & 50535.2 & 50524.6 & $4-10$ \\
\hline 1958.6 & 51056.7 & 51045.6 & $3-9$ \\
\hline
\end{tabular}

Data from McCulloh and Glockler [157b].

(b) Absorption (R)

\begin{tabular}{|c|c|c|c|}
\hline$\lambda_{H}$ & $\sigma_{H}$ & $I$ & $v^{\prime}-v^{\prime \prime}$ \\
\hline$\AA$ & $c m^{-1}$ & & \\
\hline 1510.5 & 66203 & 6 & $1-0$ \\
\hline 1478.8 & 67622 & 6 & $2-0$ \\
\hline 1449.3 & 68999 & 8 & $3-0$ \\
\hline 1421.4 & 70353 & 10 & $4-0$ \\
\hline 1395.4 & 71664 & 9 & $5-0$ \\
\hline 1370.8 & 72950 & 8 & $6-0$ \\
\hline 1347.7 & 74200 & 5 & $7-0$ \\
\hline 1326.0 & 75415 & 4 & $8-0$ \\
\hline 1305.3 & 76611 & 3 & $9-0$ \\
\hline 1286.2 & 77748 & 2 & $10-0$ \\
\hline 1267.7 & 78883 & 1 & $11-0$ \\
\hline 1250.6 & 79962 & 1 & $12-0$ \\
\hline 1234.2 & 81024 & 0.8 & $13-0$ \\
\hline$(1218.8)$ & 82048 & 0.6 & $14-0$ \\
\hline 1204.4 & 83029 & 0.5 & $15-0$ \\
\hline 1177.8 & 84904 & 0.1 & \\
\hline
\end{tabular}

Data from Tanaka, Jursa, and LeBlanc [255].
TABLE 6. Band heads and origins of the $\mathrm{B}^{1} \Sigma^{+} \rightarrow \mathrm{A}^{1} \mathrm{II}$ Angstrom system $(V)$

\begin{tabular}{|c|c|c|c|c|c|c|}
\hline$\lambda_{H}$ & $\sigma H$ & $I$ & $v^{\prime}-v^{\prime \prime}$ & Ref. & $\sigma_{0}$ & Ref. \\
\hline$\stackrel{\circ}{A}$ & $\mathrm{~cm}^{-1}$ & & & & $\mathrm{~cm}^{-1}$ & \\
\hline 6620.34 & 15100.8 & 7 & $0-5$ & 121 & 15107.2 & 121 \\
\hline 6298.5 & 15872 & 2 & $1-6$ & 23 & - & -... \\
\hline 6079.94 & 16443.0 & 9 & $0-4$ & 121 & 16449.8 & 121 \\
\hline 5817.0 & 17186 & 2 & $1-5$ & 23 & & ... \\
\hline 5610.15 & 17819.9 & 10 & $0-3$ & 121 & 17827.2 & 121 \\
\hline 5397.5 & 18522 & 2 & $1-4$ & 23 & -...... & -.- \\
\hline 5198.22 & 19232.0 & 10 & $0-2$ & 121 & 19240.3 & 121 \\
\hline 5015.0 & 19935 & 1 & $1-3$ & 23 & & -..- \\
\hline 4835.24 & 20675.74 & 10 & $0-1$ & 224 & 20682.1 & 224 \\
\hline 4697 & 21284 & 2 & $1-2$ & 23 & & -. \\
\hline 4510.86 & 22162.65 & 10 & $0-0$ & 224 & 22171.2 & 224 \\
\hline 4392. 93 & 22757.48 & 8 & $1-1$ & 224 & 22763.9 & 224 \\
\hline 4123.55 & 24244.13 & 7 & $1-0$ & 224 & 24253.7 & 224 \\
\hline
\end{tabular}

Data from Johnson and Asundi [121], Schmid and Gerö [224], and Birge [23]. The latter reference lists the band head measurements of Angstrom and Thalén which date from 1875. Intensities are primarily those of Pearse and Gaydon [187]. Duffendack and Fox [58] list a band at $4209.0 \AA$ as previously given, but this band is not further identified.

TABLE 7. Band heads and origins of the $\mathrm{B}^{1} \Sigma^{+} \rightarrow \mathrm{A}^{1} I I$ system of $\mathrm{C}^{13} \mathrm{O}^{16}(V)$

\begin{tabular}{|c|c|c|c|c|c|}
\hline$\lambda_{H}$ & $\sigma_{H}$ & $v^{\prime}-v^{\prime \prime}$ & Ref. & $\sigma_{0}$ & Ref. \\
\hline$\AA$ & $c m=1$ & & & $c m^{-1}$ & \\
\hline 6559.4 & 15241.0 & $0-5$ & $157 \mathrm{~b}$ & 15246.8 & $157 \mathrm{~b}$ \\
\hline 6038.08 & 16556.98 & $0-4$ & $157 \mathrm{a}$ & 16563.6 & $157 \mathrm{~b}$ \\
\hline 5583.06 & 17906.34 & $0-3$ & $157 \mathrm{a}$ & 17913.5 & $15 \pi \mathrm{b}$ \\
\hline 5182.71 & 19289.55 & $0-2$ & 56 & 19297.00 & 56 \\
\hline 4828.50 & 20704.57 & $0-1$ & 56 & 20712.50 & 56 \\
\hline 4396.06 & 22741.28 & $1-1$ & 56 & 22749.76 & 56 \\
\hline
\end{tabular}

Data from McCulloh [157a], McCulloh and Glockler [157b], and Douglas and $\mathrm{M} \phi$ ller [56].

TABLE 8. Band heads and origins of the $\mathrm{C}^{1} \Sigma^{+} \rightarrow \mathrm{A}^{1} \Pi$ Herzberg system $(V)$

\begin{tabular}{|c|c|c|c|c|c|c|}
\hline$\lambda_{H}$ & $\sigma_{H}$ & $I$ & $v^{\prime}-v^{\prime \prime}$ & Ref. & $\sigma_{0}$ & Ref. \\
\hline A & $\mathrm{cm}^{-1}$ & & & & $\mathrm{~cm}^{-1}$ & \\
\hline 5705.9 & 17520.9 & 0 & $0-7$ & 97 & 17520.6 & (A) \\
\hline 5318.4 & 18797.4 & 1 & $0-6$ & 97 & 18503.4 & (n) \\
\hline 4972.8 & 20103.8 & 2 & $0-5$ & 97 & 20110.2 & (*) \\
\hline 4661.68 & 21445.47 & 5 & $0-4$ & 224 & 21452.3 & (s) \\
\hline 4380.27 & 22823.22 & 7 & $0-3$ & 224 & 22830.5 & 121 \\
\hline 4125.03 & 24235.42 & 7 & $0-2$ & 224 & 24243.2 & 121 \\
\hline 3893.20 & 25678.55 & 7 & $0-1$ & 224 & 256.6 .1 & 1 \\
\hline 3680.16 & 27165.01 & 4 & $0-0$ & 224 & $2 \pi 174.17$ & $2=4$ \\
\hline
\end{tabular}

Data from Johnson and Asundi [121], Schunid and Gerö [224], and Iferzberg [97]. Intensities are those of fferzberg [97].

a Calculated from the band heads. 
TABLE 9. Band heads of the Hopfield-Birge systems

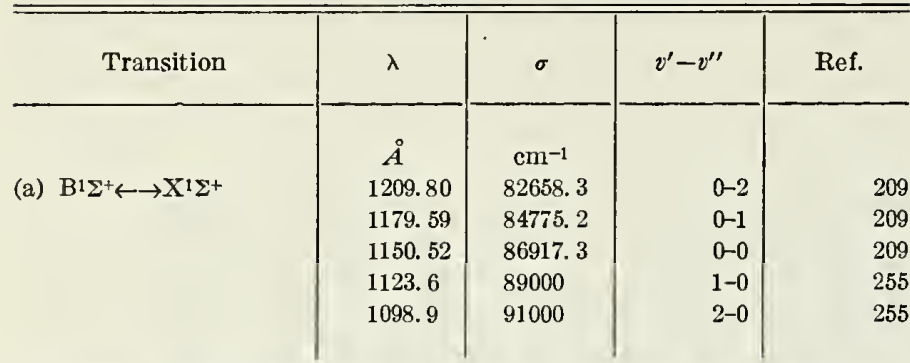

Data from Read [209] and Tanaka, Jursa, and LeBlanc [255]. The identi. fication of the $2-0$ band is uncertain.

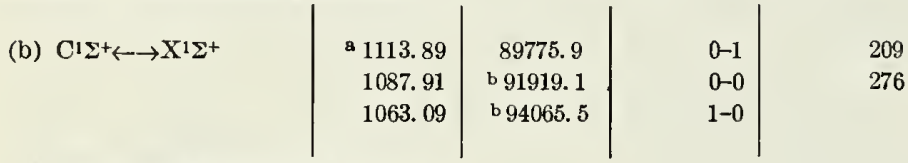

a Band head from data of Read [209].

b Band origins from [276].

(c) $\mathrm{E}^{1} \mathrm{II} \leftarrow \rightarrow \mathrm{X}^{1 \Sigma^{+}}$

$$
\mid \begin{array}{l|l}
\text { a 1076.08 } & 92930.03 \\
\text { b (1053) } & 94967
\end{array}
$$

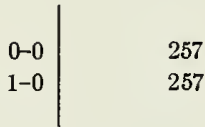

Data from Tilford, Vanderslice, and Wilkinson [257].

origin: b head.

The 0-0 band head has been observed [255] at $1076.1 \AA, 92928 \mathrm{~cm}^{-1}$.

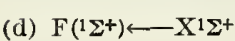

\begin{tabular}{|l|r|}
1002.7 & 99730 \\
a $(985.8)$ & 101450 \\
a $(973.1)$ & 102770 \\
&
\end{tabular}

\begin{tabular}{r|}
$0-0$ \\
$1-0$ \\
$2-0$
\end{tabular}

104

117

117

Data from Hopfield and Birge [104] and Jevons [117].

a 1-0, 2-0 band positions inferred from vibrational constants listed by Jevons [117] and Herzberg [99].

(e) $\mathrm{G}(1 \Pi) \longleftarrow \mathrm{X}^{1} \Sigma^{+}$ \begin{tabular}{l|l}
949.97 & 105266
\end{tabular}<smiles>[TeH]</smiles>
104

Data from Hopfield ancl Birge [104].

(f) $\mathrm{b}^{3} \Sigma^{+} \longleftrightarrow \mathrm{X}^{1 \Sigma^{+}}$
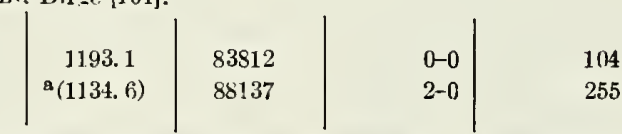

Data from Hopfieid and Birge [104] and Tanaka, Jursa, and LeBlanc [255]. Her zberg [99] lists (1192. $9 \AA$ ) , $83831 \mathrm{~cm}^{-1}$ for $0-\mathrm{u}$.

a Uncertain identification of a very weak band. No other observation of $b, v=2$ has been reported.
TABLE 10. Band heads and origins of the $\mathrm{a}^{\prime} \Sigma^{+} \leftarrow \mathrm{X}^{1} \Sigma^{+}$ Hopfield-Birge system $(R)$

\begin{tabular}{|c|c|c|c|c|}
\hline$\lambda_{\mathrm{H}}$ & $\sigma_{\mathrm{H}}$ & $\sigma_{0}$ & $I$ & $v^{\prime}-v^{\prime \prime}$ \\
\hline$\AA$ & $\mathrm{cm}^{-1}$ & $\mathrm{~cm}-\mathrm{l}$ & & \\
\hline 1806. 3 & 55363 & 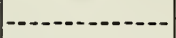 & 0 & $0-0$ \\
\hline 1767.7 & 56570 & - & 0 & $1-0$ \\
\hline 1731.4 & 57756 & 57750.16 & 1 & $2-0$ \\
\hline 1697.2 & 58922 & 58916.49 & 2 & $3-0$ \\
\hline 1664.8 & 60068 & 60062.64 & 4 & $4-0$ \\
\hline 1634.2 & 61192 & 61187.65 & 8 & $5-0$ \\
\hline 1605.2 & 62299 & 62293.64 & 20 & $6-0$ \\
\hline 1577.7 & 63384 & 63379.44 & 40 & $7-0$ \\
\hline - & - n... & a (64446. 9) & $\ldots$ & $8-0$ \\
\hline 1526.8 & 65495 & 65492.28 & 80 & $9-0$ \\
\hline 1503.2 & 66523 & 66520.39 & 100 & $10-0$ \\
\hline - & - - & a (67529.5) & ----- & $11-0$ \\
\hline 1459.4 & 68522 & 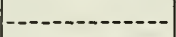 & 80 & $12-0$ \\
\hline 1439.0 & 69494 & 69491.25 & 100 & $13-0$ \\
\hline - & ...... & - & 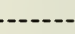 & $14-0$ \\
\hline - & -- - & 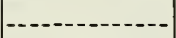 & $\ldots$ & $15-0$ \\
\hline 1383.2 & 72298 & 72295.14 & 50 & $16-0$ \\
\hline 1366. 2 & 73194 & |--non & 50 & $17-0$ \\
\hline & & & & $18-0$ \\
\hline 1334.5 & 74934 & 74932.04 & 30 & $19-0$ \\
\hline 1319.7 & 75776 & 75773.91 & 20 & $20-0$ \\
\hline -..-- & -- & - & & $21-0$ \\
\hline 1291.9 & 77408 & $4-2-2-2$ & 20 & $22-0$ \\
\hline 1279.0 & 78186 & 78184.8 & 10 & $23-0$ \\
\hline
\end{tabular}

Data from Herzberg and Hugo [101].

a Data from Gerö and Lörinczi [80]. 
TABLE 11. Band heads of the $\mathrm{a}^{3} \mathrm{I}-\mathrm{X}^{1} \Sigma^{+}$Cameron syslem $(R)$

(a) Emission

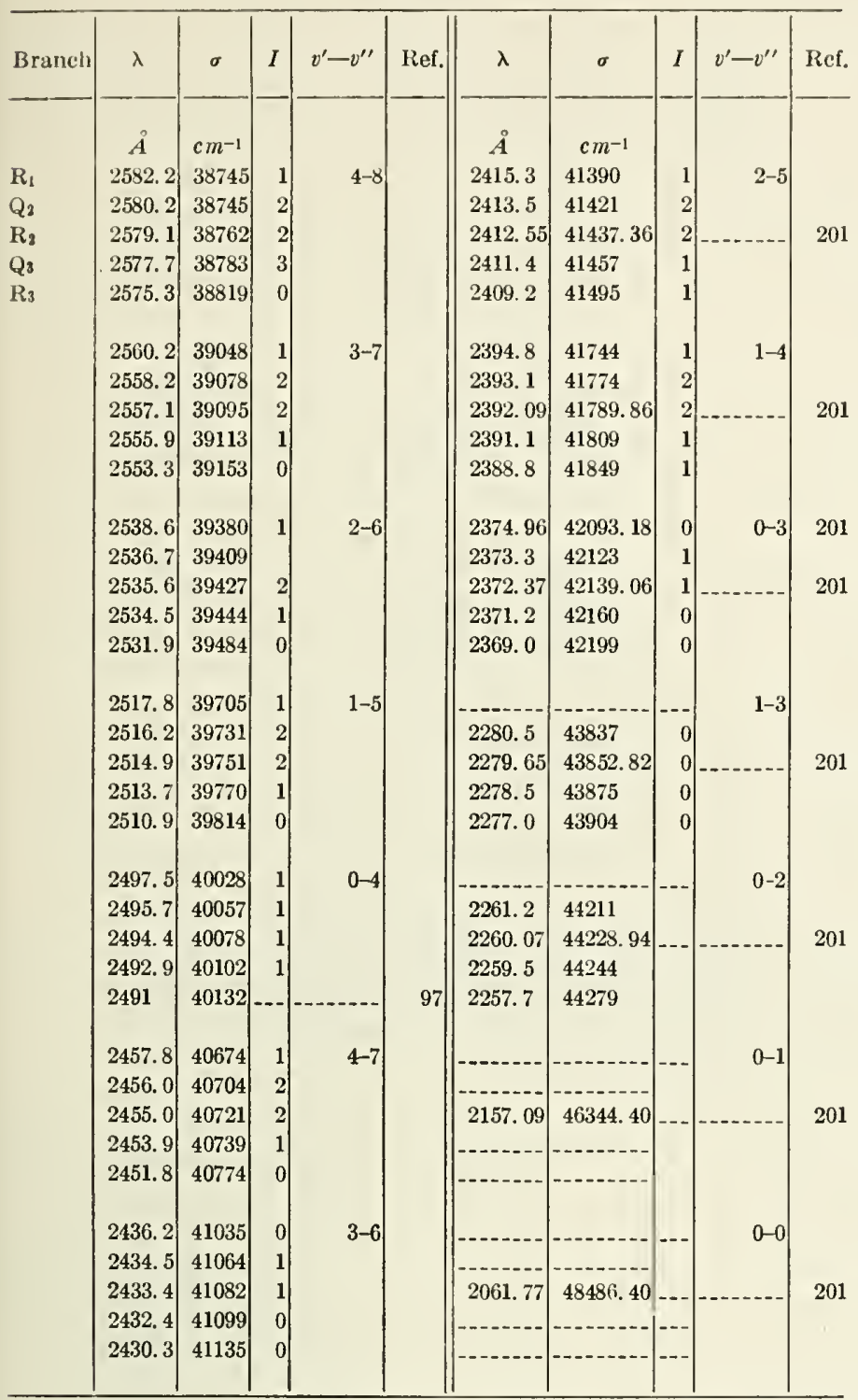

Most of the data are from Cameron [39] supplemented by data from Rao [201] and Herzberg [97]. Intensities are from Cameron [39]. Wavelengths of Cameron [39] are uncertain by $0.5 \AA$ [119].
TABLE 11. Band heads of the $\mathrm{a}^{3} \mathrm{II}-\mathrm{X}^{1} \Sigma^{+}$Cameron system $(R)$-Continued

(b) Absorption a

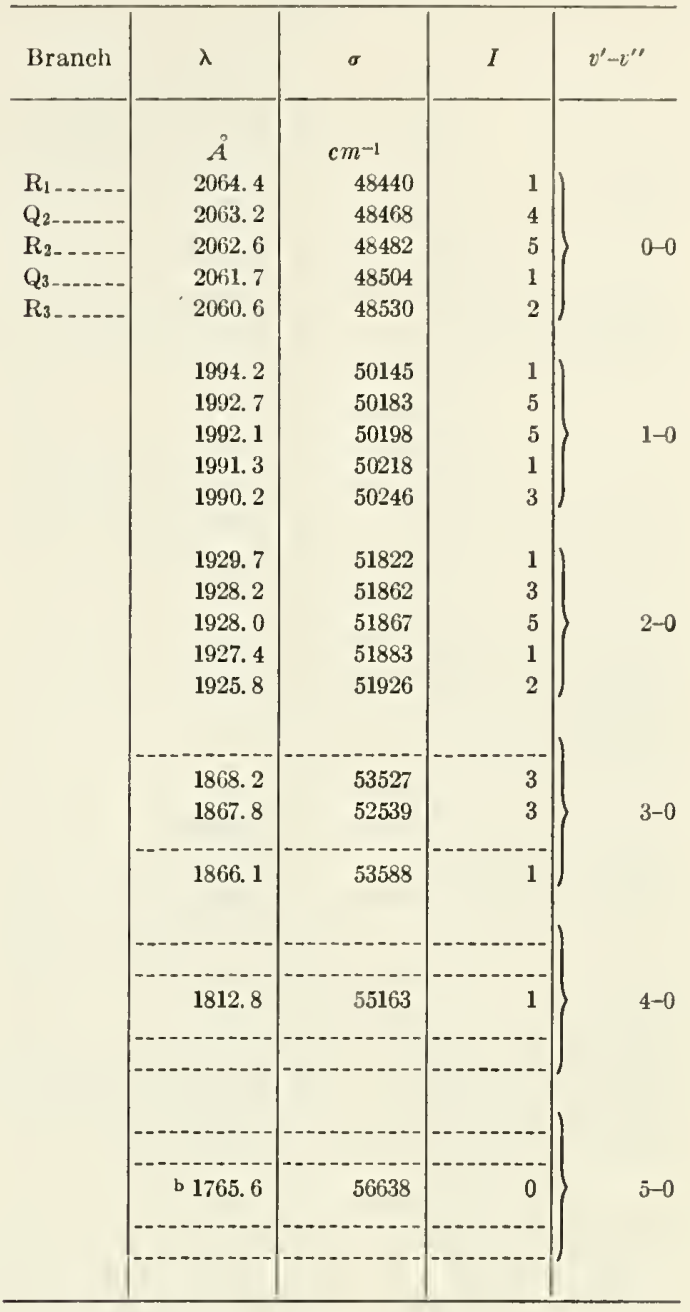

B All vacuum wavelengths.

b Possibly incorrect branch designation.

Data taken from Tanaka, Jursa, and LeBlanc [255].

TABLE 12. Band origins of the $\mathrm{a}^{3} \Pi-\mathrm{X}^{1} \Sigma^{+}$Cameron system

\begin{tabular}{|c|c|c|}
\hline$\sigma_{0}$ & $v^{\prime}-v^{\prime \prime}$ & Ref. \\
\hline $\mathrm{cm}^{-1}$ & & \\
\hline 50188.3 & $1-0$ & 79 \\
\hline 48473.97 & $0-0$ & 201 \\
\hline 46330.59 & $0-1$ & 201 \\
\hline 44213.85 & $0-2$ & 201 \\
\hline 43838.19 & $1-3$ & 20 \\
\hline 42123.58 & $0-3$ & 201 \\
\hline 41774.18 & $1-4$ & 201 \\
\hline
\end{tabular}

Data for 1-0 band from Gicrö, Herzberg, and Schmid [79]; all other data from Rao [201]. 
TABLE 13. Band heads of the $\mathrm{b}^{3} \Sigma^{+} \rightarrow \mathrm{a}^{3} \Pi$ Third Positive system $(V)$

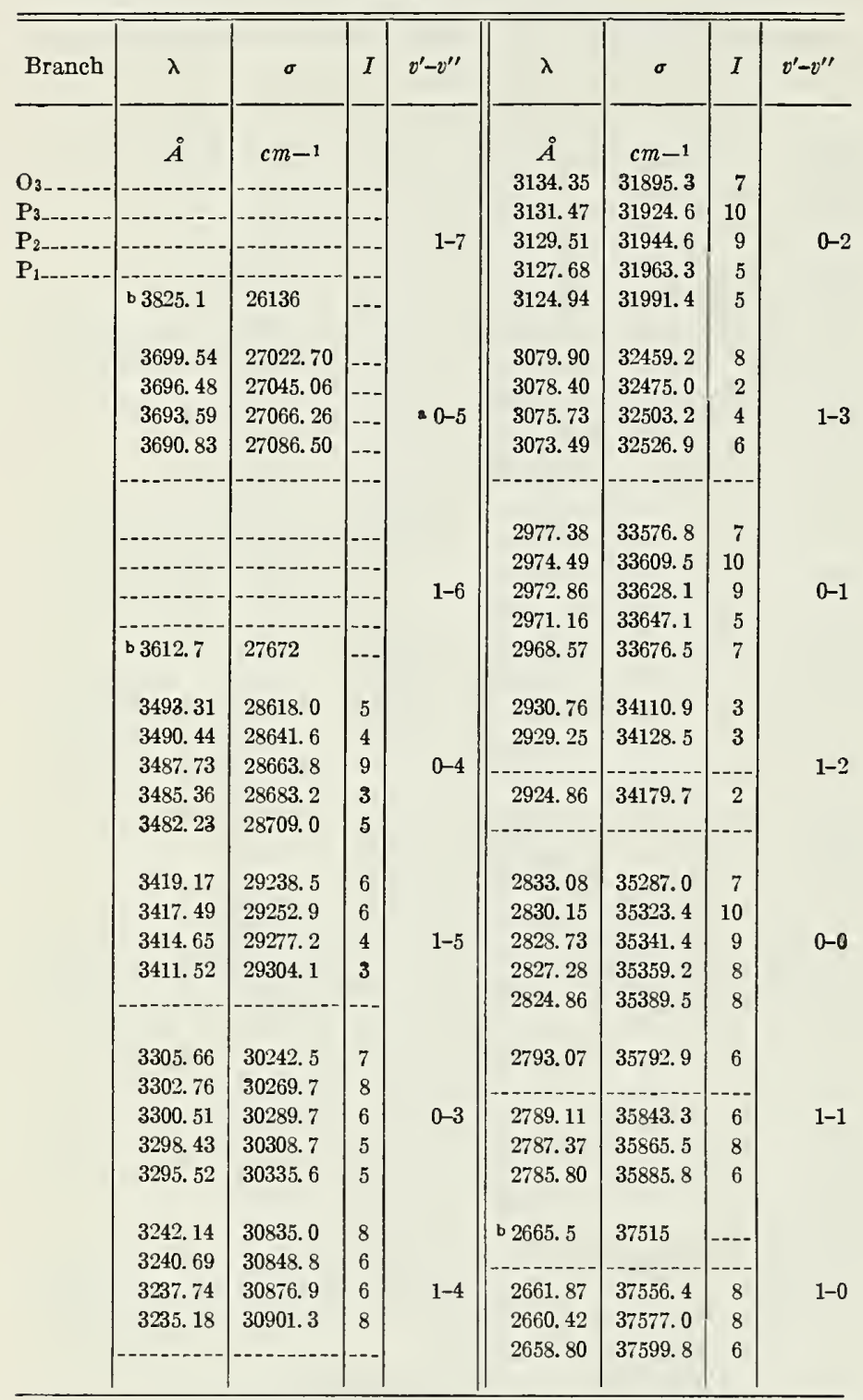

Band heads mostly from Asundi [4].

a Data on 0-5 band from Beer [16].

bData from Duffendack and Fox [57].
$\mathrm{T}_{\triangle \mathrm{BLE}}$ 14. Band heads of the $\mathrm{c}^{3} \Sigma^{+} \rightarrow \mathrm{a}^{3} \Pi 3 A$ system $(V)$

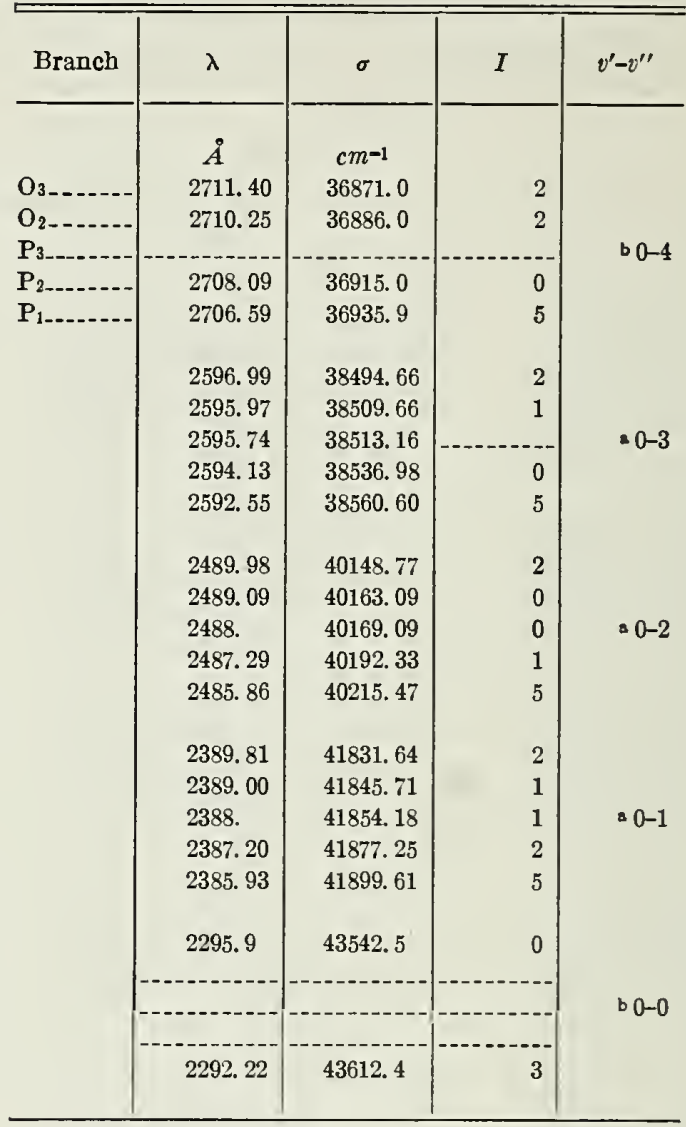

-Data from the fine structure work of Gerö [77].

bData from vibrational measurements of Asundi [4].

For the 0-3, 0-2, and 0-1 bands, both authors' data agree to within $0.3 \AA$. These bands appear double-headed under low dispersion. 
TABLE 15. Band heads of the $\mathrm{a}^{\prime}{ }^{3} \Sigma^{+} \rightarrow \mathrm{a}^{2} \Pi$ Asundi system $(R)$

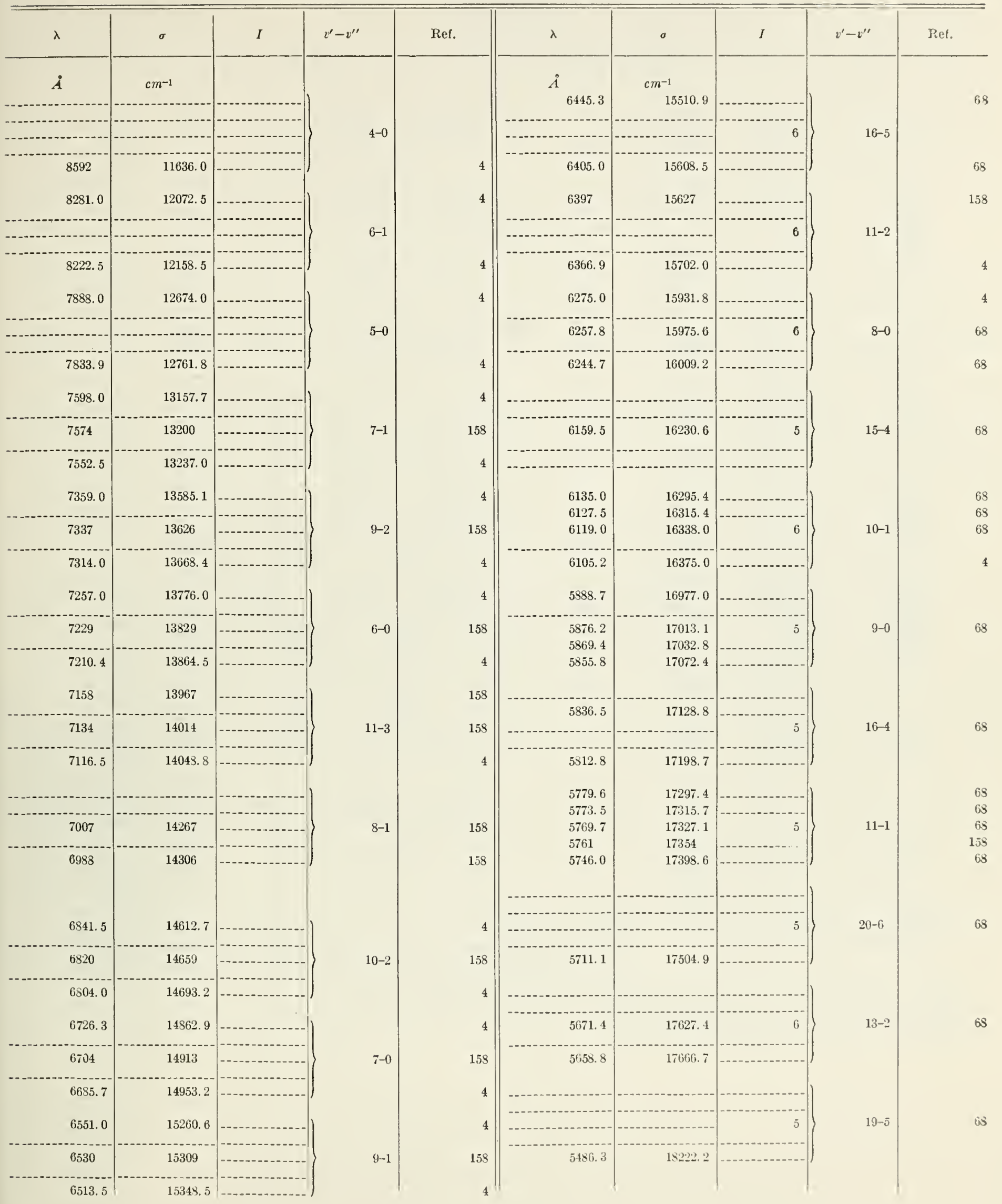

47 
TABLE 15. Band heads of the $\mathbf{a}^{\prime 3} \Sigma^{+} \rightarrow \mathbf{a}^{3} \Pi$ Asundi system ( $R$ )-Continued

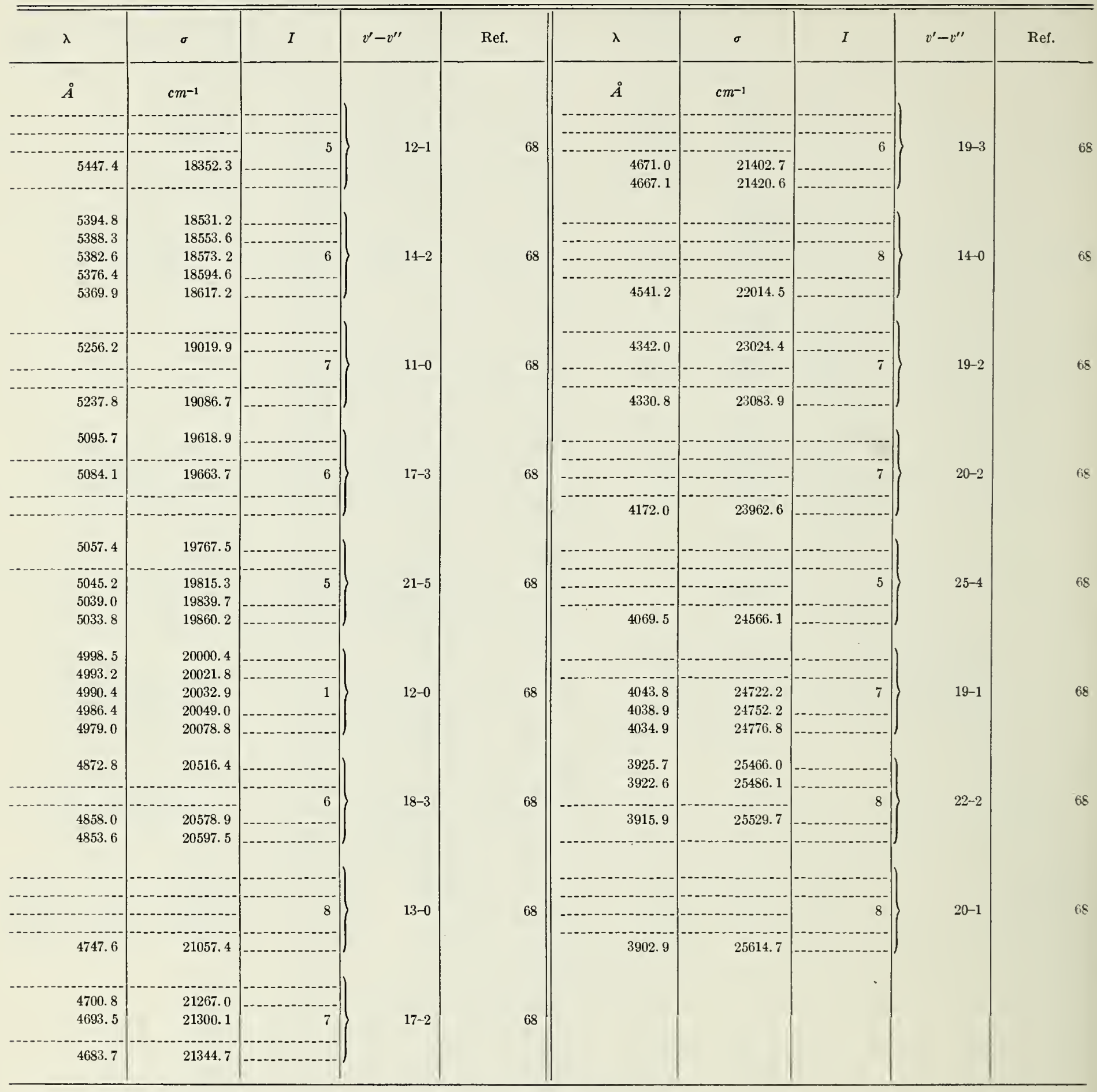

Data from Garg [68] Asundi [4], and McLennan, Smith, and Peters [158]. Intensities from Garg [68].

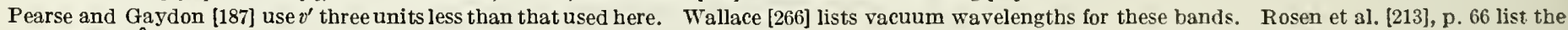
band at $6685.7 \AA$ as $7-1$; it is the ${ }^{T} R_{31}$ head of the $7-0$ band. 
TABLE 16. Band heads of the $\mathrm{d}^{3} \Delta_{i} \rightarrow \mathrm{a}^{3} \Pi$ Triplet system $(R)$

(a) Identified bands

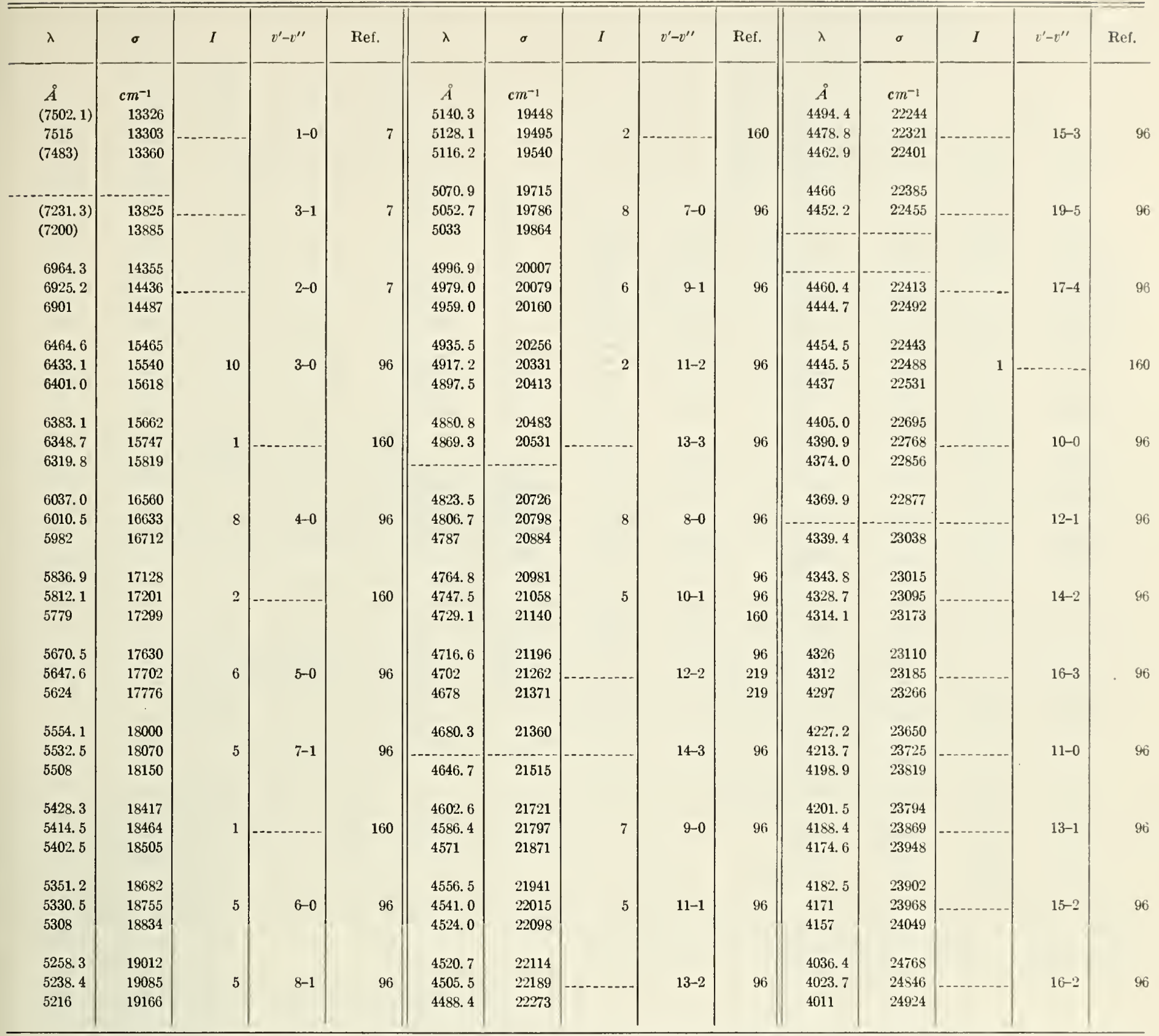

Data from Herman and Herman [96, 92] supplemented by data from Merton and Johnson [160] and Asundi [7]. Intensities are from Merton and Johnson

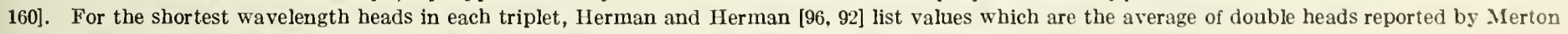
and Johnson [160]. Numerous other band positions of reference 96 are exactly those given in ref. [160]. 
TABLE 16. Band heads of the $\mathrm{d}^{6} \Delta_{i} \rightarrow \mathrm{a}^{3} \Pi$ Triplet system (R)-Continued

(b) Unassigned bands a

\begin{tabular}{|c|c|c|c|c|c|}
\hline$\lambda$ & $\sigma$ & $\lambda$ & $\sigma$ & $\lambda$ & $\sigma$ \\
\hline$\AA$ & $c m^{-1}$ & $\AA$ & $c m^{-1}$ & $\AA$ & $c m^{-1}$ \\
\hline 6613 & 15118 & 4460.1 & 22415 & 4074 & 24540 \\
\hline 6557 & 15247 & 4441.7 & 22508 & & -...-. \\
\hline 6515 & 15345 & $\cdots$ & & & --..- \\
\hline 6323 & 15811 & 4460 & 22415 & 4055 & 24655 \\
\hline 6282 & 15914 & 4444 & 22495 & 4049 & 24690 \\
\hline 6248 & 16001 & 4437 & 22531 & 4034 & 24780 \\
\hline 6141 & 16279 & 4428 & 22575 & 4034 & 24780 \\
\hline 6122 & 16330 & 4416 & 22640 & 4023 & 24850 \\
\hline 6105 & 16375 & 4406 & 22690 & 4010 & 24930 \\
\hline 5889 & 16976 & 4398 & 22730 & 3928 & 25450 \\
\hline 5860 & 17060 & 4378 & 22835 & 3919 & 25510 \\
\hline 5839 & 17121 & 4370 & 22875 & 3912 & 25555 \\
\hline 5804 & 17225 & 4314 & 23175 & 3896 & 25660 \\
\hline 5777 & 17305 & 4297 & 23265 & 3893 & 25680 \\
\hline 5752 & 17380 & 4288 & 23315 & 3884 & 25740 \\
\hline 5157 & 19386 & 4240 & 23580 & 3885 & 25735 \\
\hline 5138 & 19457 & 4229 & 23640 & 3875 & 25800 \\
\hline 5112 & 19556 & 4213 & 23730 & 3870 & 25830 \\
\hline 5098 & 19610 & 4344 & 23015 & 3804 & 26280 \\
\hline & & 4339 & 23040 & 3795 & 26345 \\
\hline & 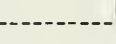 & 4331 & 23085 & 3788 & 26390 \\
\hline 4888 & 20453 & 4188 & 23870 & 3778 & 26460 \\
\hline 4870 & 20528 & 4184 & 23895 & 3767 & 26540 \\
\hline 4850 & 20613 & 4176 & 23940 & & \\
\hline 4663 & 21439 & 4161 & 24025 & 3755 & 266 \\
\hline 4647 & 21513 & 4156 & 24055 & 3740 & 26730 \\
\hline 4625 & 21616 & 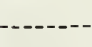 & & & \\
\hline 4491 & 22260 & 4115 & 24295 & & \\
\hline 4480 & 22315 & 4108 & 24335 & & \\
\hline 4463 & 22400 & 4088 & 24455 & & \\
\hline
\end{tabular}

a Data from Sato [219] who assigned quantum numbers to these bands. Those assignments are considered uncertain.

TABLE 17. Band origins of the $\mathrm{d}^{3} \Delta_{1} \rightarrow \mathrm{a}^{3} \Pi$ Triplet system $(R)$

\begin{tabular}{l|r}
\hline \hline$\sigma 0$ & $v^{\prime}-v^{\prime \prime}$ \\
\hline$c m^{-1}$ & \\
15538.5 & $3-0$ \\
16627.4 & $4-0$ \\
19785.8 & $7-0$ \\
21794.4 & $9-0$ \\
&
\end{tabular}

Data from Gerö and Szabó [82] with revised $v^{\prime}$ numbering of Carroll [40].
TABLE 18. Band heads and origins of the $\mathrm{e}^{3} \Sigma^{-} \leftarrow \mathrm{X}^{1} \Sigma^{+}$ system $(R)$

\begin{tabular}{|c|c|c|c|c|}
\hline$\lambda$ & $\sigma_{H}$ & $\sigma_{0}$ & $I$ & $v^{\prime}-v^{\prime \prime}$ \\
\hline$\AA$ & $c m^{-1}$ & $\mathrm{~cm}^{-1}$ & & \\
\hline 1542.8 & 64816 & 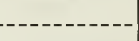 & 50 & $1-0$ \\
\hline 1517.7 & 65891 & 65877.82 & 30 & $2-0$ \\
\hline 1493.7 & 66946 & 66933.26 & 20 & $3-0$ \\
\hline 1471.0 & 67982 & 67970.31 & 50 & $4-0$ \\
\hline 1428.9 & 69992 & 69986. 39 & 30 & $6-0$ \\
\hline 1390.1 & 71925 & 71926.47 & 25 & $8-0$ \\
\hline 1355.2 & 73792 & 73792.28 & 20 & $10-0$ \\
\hline 1338.5 & 74709 & -------- & 20 & $11-0$ \\
\hline 1308.1 & $76448 ?$ & & 20 & $13-0$ \\
\hline 1293.6 & $77303 ?$ & & 15 & $14-0$ \\
\hline 1241.9 & $80523 ?$ & & 5 & $18-0$ \\
\hline
\end{tabular}

Data from Herzberg and Hugo [101]. All $v^{\prime}$ values are one unit larger than in the original paper [238a].

(?) Band heads seen at expected positions. Bands with $v^{\prime}$ up to 23 have been observed [238a] but detailed analysis is not yet available.

TABLE 19. Band heads of the $\mathrm{C}^{1} \Sigma^{+} \rightarrow \mathrm{a}^{\prime 3} \Sigma^{+}$Knauss system $(\mathrm{V})$

\begin{tabular}{|c|c|c|}
\hline$\lambda$ & $\sigma$ & $v^{\prime}-v^{\prime \prime}$ \\
\hline$\AA$ & $\mathrm{cm}-1$ & \\
\hline 3253 & 30732 & $0-5$ \\
\hline 3138 & 31858 & $0-4$ \\
\hline 3028 & 33015 & $0-3$ \\
\hline 2925 & 34178 & $0-2$ \\
\hline
\end{tabular}

Data from Knauss [130].

TABLE 20. Band heads of the Kaplan system $(R)$ s

\begin{tabular}{|c|c|c|c|c|}
\hline Transition & $\lambda$ & $\sigma$ & $v^{\prime}-v^{\prime \prime}$ & Ref. \\
\hline \multirow{4}{*}{$\mathrm{K}-a^{3} \Pi$} & & $\mathrm{cm}^{-1}$ & & \\
\hline & 2750 & 36350 & $0-3$ & 125 \\
\hline & 2630 & 38010 & $0-2$ & 125 \\
\hline & 2518 & 39700 & $0-1$ & 125 \\
\hline$?-\mathrm{X}^{1} \Sigma^{+}$ & ${ }^{\mathrm{a}}(2575)$ & 38820 & & 126 \\
\hline
\end{tabular}

a The reality of this band as representing a transition in $\mathrm{CO}$ is highly questionable. 
TABLE 21. Band heads of the $\mathrm{d}^{3} \Delta_{i} \leftarrow \mathrm{X}^{1} \Sigma^{+}$system $(R)^{\mathrm{a}}$

\begin{tabular}{|c|c|c|c|}
\hline$\lambda$ & $\sigma$ & $I$ & $v^{\prime}-v^{\prime \prime}$ \\
\hline$\stackrel{\circ}{ }$ & $\mathrm{cm}^{-1}$ & & \\
\hline 1617.5 & 61824 & $0^{+}$ & $1-0$ \\
\hline 1588.8 & 62941 & 1 & $2-0$ \\
\hline 1561.3 & 64049 & 3 & $3-0$ \\
\hline 1535.2 & 65138 & 3 & 4-0 \\
\hline 14866 & 67268 & $=--$ & $6-0$ \\
\hline 1464.2 & 68297 & 5 & $7-0$ \\
\hline 1442.8 & 69310 & 6 & $8-0$ \\
\hline - & $-1-1$ & --- & \\
\hline 1402.8 & 71286 & 10 & $10-0$ \\
\hline 1384.2 & 72244 & 10 & $11-0$ \\
\hline 1366.6 & 73174 & 9 & $12-0$ \\
\hline 1350.0 & 74074 & 7 & $13-0$ \\
\hline 1334.8 & 74918 & 5 & $14-0$ \\
\hline 1319.6 & 75781 & 5 & $15-0$ \\
\hline 1305.3 & 76611 & 3 & $16-0$ \\
\hline 1291.9 & 77405 & 3 & $17-0$ \\
\hline 1279.0 & 78186 & 2 & $18-0$ \\
\hline 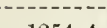 & & & \\
\hline 1254.4 & 79719 & 1 & $20-0$ \\
\hline 1243.4 & 80425 & $0^{+}$ & $21-0$ \\
\hline 1232.4 & 81142 & $0^{+}$ & $22-0$ \\
\hline
\end{tabular}

Data from Tanaka, Jursa, and LeBlanc [255]. Their $v^{\prime}$ numbering has been raised one unit [40]; the upper state had formerly been considered ' $\Pi_{i}\{40,171\}$.

a Band intensities are almost $10^{3}$ times weaker than the $\mathrm{A}-\mathrm{X}$ bands.

TABLE 22. Band heads of the $\mathrm{e}^{3} \Sigma^{-} \rightarrow \mathrm{a}^{3} \Pi$ Herman system $(R)$

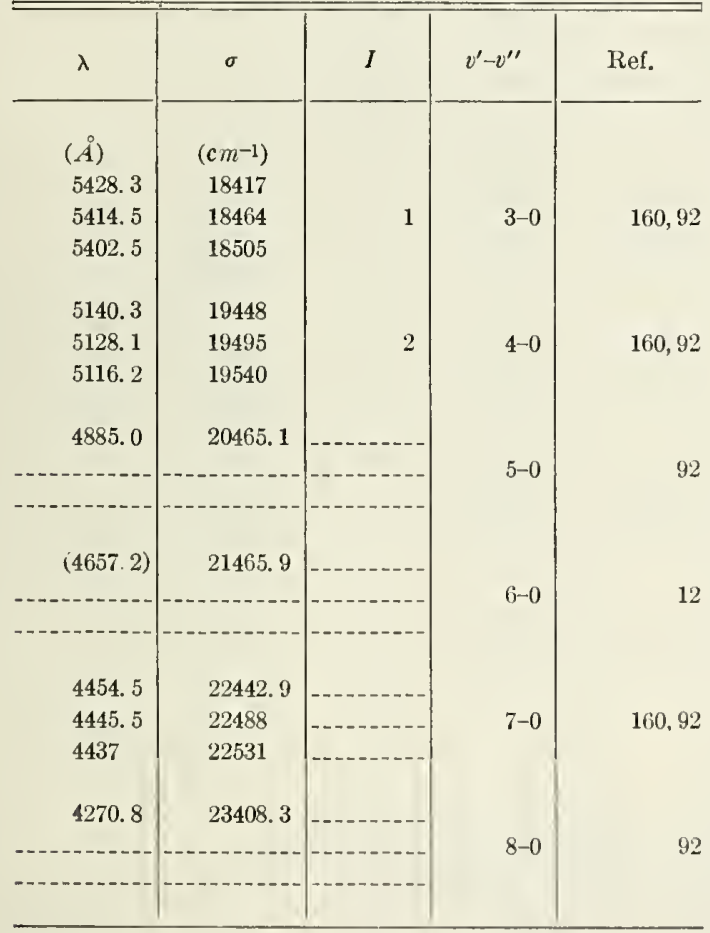

( ) = interpolated.

See also ref. [96]. The intensities are those given by Merton and Johnson [160]. Revised $v^{\prime}$ quantum numbering is that suggested by Siminons et al., [238a].
TABLE 23. Band heads of the $\mathrm{F}_{00}{ }^{1} \Sigma \rightarrow \mathrm{X}^{1} \Sigma^{+}$system $(R)$

\begin{tabular}{|c|c|c|c|}
\hline$\lambda$ & $\sigma$ & $I$ & $v^{\prime}-v^{\prime \prime}$ \\
\hline A & $\mathrm{cm}^{-1}$ & & \\
\hline 1309.13 & 76386.4 & 1 & $0-7$ \\
\hline 1276.07 & 78365.9 & 2 & $0-6$ \\
\hline 1244.16 & 80375.5 & 3 & $0-5$ \\
\hline 1213.31 & 82419.2 & 4 & $0-4$ \\
\hline 1183.62 & 84486.6 & 5 & $0-3$ \\
\hline 1154.91 & 86586.9 & 4 & $0-2$ \\
\hline 1127.23 & a 88713.0 & 2 & $0-1$ \\
\hline 1100.49 & 90868.6 & 2 & $0-0$ \\
\hline
\end{tabular}

Data from Tschulanovsky [260]. The formula fitting the term values of these bands deviates considerably from that of the ground state. This makes the reality of the $\mathrm{E}_{0}$ state somewhat uncertain. In this report $v^{\prime}=0$ has been assumed.

a Band also observed in absorption by Tanaka, Jursa, and LeBlane [255].

TABLE 24. Band heads of the ${ }^{1} \Pi \rightarrow \mathrm{X}^{1} \Sigma^{+}$system $(R)$

\begin{tabular}{c|r|r|r}
\hline$\lambda$ & $\sigma$ & $I$ & $\sigma^{\prime}-v^{\prime \prime}$ \\
\hline & & & \\
\hline$A$ & $c m^{-1}$ & & \\
1132.52 & 88299 & 1 & $0-5$ \\
1106.44 & 90380 & 2 & $0-4$ \\
1081.77 & 92441 & & $0-3$ \\
1057.60 & 94554 & 4 & $0-2$ \\
1034.65 & 96651 & 3 & $0-1$ \\
1011.75 & 98839 & 1 & $0-0$ \\
& & & \\
\hline
\end{tabular}

Data from Tschulanovsky [260].

TABLE 25. Band heads of the $\mathrm{f}^{3} \Sigma^{+} \rightarrow \mathrm{a}^{3}$ II system $(R)$

\begin{tabular}{c|c|r}
\hline$\lambda$ & $\sigma$ & $v^{\prime}-v^{\prime \prime}$ \\
\hline$\AA$ & $c m^{-1}$ & \\
2979.95 & 33547.82 & $0-1$ \\
2670.14 & 37440.15 & $?-0$ \\
& & \\
\hline
\end{tabular}

Data from Gerö [78]. The $v^{\prime}$ numbering is uncertain. 
TABLE 26. Band heads of Tanaka systems (absorption)

(a) $\mathrm{P} \leftarrow \mathrm{X}^{1} \Sigma^{+}$

\begin{tabular}{c|c|c|c}
\hline$\lambda$ & $\sigma$ & $I$ & $v^{\prime}-v^{\prime \prime}$ \\
\hline$A$ & $c m^{-1}$ & & \\
791.08 & 126410 & 5 & $0-0$ \\
781.50 & 127959 & 6 & $1-0$ \\
772.32 & 129480 & 6 & $2-0$ \\
763.68 & 130945 & 5 & $3-0$ \\
755.24 & 132408 & 4 & $4-0$ \\
747.30 & 133815 & 4 & $5-0$ \\
739.52 & 135223 & 2 & $6-0$ \\
732.00 & 136612 & 3 & $7-0$ \\
\hline
\end{tabular}

(b) $\mathrm{Q} \leftarrow \mathrm{X}^{1} \Sigma^{+}$

\begin{tabular}{l|l|l|l}
\hline 776.77 & 128738 & 2 & $0-0$ \\
767.60 & 130276 & 5 & $1-0$ \\
758.80 & 131787 & 3 & $2-0$ \\
750.22 & 133294 & 3 & $3-0$ \\
742.09 & 134755 & 3 & $4-0$ \\
734.15 & 136212 & 2 & $5-0$ \\
\hline
\end{tabular}

TABLE 26. Band heads of Tanaka systems (absorption)Continued

(c) $\mathrm{R} \leftarrow \mathrm{X}^{1} \Sigma^{+}$

\begin{tabular}{l|l|l|l}
\hline 776.00 & 128866 & 3 & $0-0$ \\
766.71 & 130427 & 3 & $1-0$ \\
757.86 & 131950 & 3 & $2-0$ \\
749.48 & 133426 & 2 & $3-0$ \\
741.40 & 134880 & 2 & $4-0$ \\
733.36 & 136359 & 4 & $5-0$ \\
\hline
\end{tabular}

(d) $\mathrm{S} \leftarrow \mathrm{X}^{1} \Sigma^{+}$

\begin{tabular}{l|l|l|l}
\hline 690.92 & 144735 & 2 & $0-0$ \\
683.21 & 146368 & 1 & $1-0$ \\
675.73 & 147988 & 1 & $2-0$ \\
668.44 & 149602 & 0 & $3-0$ \\
\hline
\end{tabular}

(e) $\mathrm{T} \leftarrow \mathrm{X}^{1} \Sigma^{+}$

\begin{tabular}{l|l|l|l}
\hline 647.83 & 154362 & 3 & $0-0$ \\
642.27 & 155698 & 2 & $1-0$ \\
636.88 & 157016 & 1 & $2-0$ \\
\hline
\end{tabular}

Data taken from Tanaka [254].

- Misprint in original paper corrected.

TABLE 27. $\mathrm{B}^{2} \Sigma^{+}\left(\mathrm{CO}^{+}\right) \leftarrow \mathrm{X}^{1} \Sigma^{+}$" $\beta^{\prime \prime}$ Rydberg series

\begin{tabular}{|c|c|c|c|c|c|c|c|c|c|}
\hline \multirow{2}{*}{$n$} & \multirow{2}{*}{$\lambda$} & \multicolumn{2}{|c|}{ Sharp series } & \multirow{2}{*}{$v^{\prime}-v^{\prime \prime}$} & \multirow{2}{*}{$n$} & \multicolumn{2}{|c|}{ Diffuse series } & \multirow{2}{*}{$I$} & \multirow{2}{*}{$v^{\prime}-v^{\prime \prime}$} \\
\hline & & $\sigma$ & $I$ & & & $\lambda$ & 厂 & & \\
\hline \multirow{3}{*}{4} & A & $c m^{-1}$ & & & \multirow{3}{*}{4} & A & $\mathrm{cm}^{-1}$ & & \\
\hline & s 725.9 & 137760 & -..... & $0-0$ & & 716.7 & 139530 & $\ldots$ & $0-0$ \\
\hline & 672.44 & 148712 & 5 & $0-0$ & & 670.67 & 149105 & 4 & $0-0$ \\
\hline \multirow{4}{*}{5} & 664.87 & 150405 & 4 & $1-0$ & \multirow[t]{4}{*}{5} & 663.32 & 150757 & 2 & $1-0$ \\
\hline & 657.67 & 152053 & 3 & $2-0$ & & 656.40 & 152346 & 1 & $2-0$ \\
\hline & 650.81 & 153655 & 0 & $3-0$ & & & & & \\
\hline & 654.40 & 152812 & 4 & $0-0$ & & 653.58 & 153004 & 2 & $0-0$ \\
\hline \multirow{4}{*}{6} & 647.22 & 154507 & 2 & $1-0$ & \multirow[t]{4}{*}{6} & 646.53 & 154672 & 1 & $1-0$ \\
\hline & 640.35 & 156165 & $0^{+}$ & $2-0$ & & 639.84 & 156289 & 1 & $2-0$ \\
\hline & 633.80 & 157779 & $0^{+}$ & $3-0$ & & & & & \\
\hline & 645.91 & 154820 & 2 & $0-0$ & & 645.40 & 154943 & 2 & $0-0$ \\
\hline \multirow[t]{3}{*}{7} & 639.13 & 156463 & 2 & $1-0$ & \multirow[t]{2}{*}{7} & 638.56 & 156602 & 1 & $1-0$ \\
\hline & 632.43 & 158120 & $0^{+}$ & $2-0$ & & 631.98 & 158233 & $0^{+}$ & $2-0$ \\
\hline & 641.26 & 155943 & 2 & $0-0$ & 8 & 640.81 & 156053 & 1 & $0-0$ \\
\hline \multirow[t]{2}{*}{8} & 634.41 & 157627 & 1 & $1-0$ & \multirow{7}{*}{9} & \multirow{7}{*}{638.05} & \multirow{7}{*}{156728} & \multirow{7}{*}{1} & \multirow{7}{*}{$0-0$} \\
\hline & 628.05 & 159223 & -.- & $2-0$ & & & & & \\
\hline 9 & 638.42 & 156637 & 2 & $0-0$ & & & & & \\
\hline 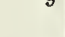 & 631.72 & 158298 & $0^{+}$ & $1-0$ & & & & & \\
\hline 10 & 636.61 & 157082 & 1 & $0-0$ & & & & & \\
\hline 11 & 635.26 & 157416 & 1 & $0-0$ & & & & & \\
\hline 12 & 634.41 & 157627 & 0 & $0-0$ & & & & & \\
\hline
\end{tabular}

Data taken from Tanaka [254].

The diffuse series is possibly (1II) $\leftarrow \mathrm{X}^{1 \Sigma}+[254]$.

a Data for $n=4$ taken from Huffman, Larrabee, and $\sigma_{\infty}($ sharp $)=158692 \pm 30 \mathrm{~cm}^{-1}=19.6748 \mathrm{eV}=630.16 \AA$. Tanaka [107]; wave-lengths to $\pm 0.5 \AA$.

The sharp series is possibly $(1 \Sigma) \leftarrow X \mid \Sigma+[254]$.

$\sigma_{\infty}($ diffuse $)=158745 \pm 30 \mathrm{~cm}^{-1}=19.6814 \mathrm{eV}=629.94 \AA$ 
TABLE 28. $\mathrm{A}^{2} \mathrm{II}_{i}\left(\mathrm{CO}^{+}\right) \leftarrow \mathrm{N}^{1} \Sigma^{+}$" $\alpha$ " Rydberg series

\begin{tabular}{|c|c|c|c|c|}
\hline$n$ & $\lambda$ & $\sigma$ & $I$ & $v^{\prime}-v^{\prime \prime}$ \\
\hline \multirow{7}{*}{5} & $\AA$ & $c m^{-1}$ & & \\
\hline & 810.80 & 123335 & 3 & $0-0$ \\
\hline & 800.94 & 124856 & 3 & $1-0$ \\
\hline & 791.64 & 126320 & 4 & $2-0$ \\
\hline & 782.47 & 127800 & 3 & $3-0$ \\
\hline & 773.76 & 129239 & 3 & $4-0$ \\
\hline & 784. 70 & 127436 & 3 & $0-0$ \\
\hline \multirow{4}{*}{6} & 775.11 & 129014 & 3 & $1-0$ \\
\hline & 766.09 & 130533 & 4 & $2-0$ \\
\hline & 757.35 & 132039 & 3 & $3-0$ \\
\hline & 772.61 & 129431 & 6 & $0-0$ \\
\hline \multirow[t]{3}{*}{7} & 763.50 & 130975 & 7 & $1-0$ \\
\hline & 754.70 & 132503 & 3 & $2-0$ \\
\hline & 765.35 & 130659 & 2 & $0-0$ \\
\hline \multirow[t]{3}{*}{8} & 756.37 & 132210 & 2 & $1-0$ \\
\hline & 747. 74 & 133736 & 2 & $2-0$ \\
\hline & 761.48 & 131323 & 1 & $0-0$ \\
\hline \multirow[t]{2}{*}{9} & 752.60 & 132873 & 2 & $1-0$ \\
\hline & 744.03 & 134403 & 1 & $2-0$ \\
\hline
\end{tabular}

Data froon Tanaka [254].

$\sigma_{\infty}=133380 \mathrm{em}^{-1}=16.537 \mathrm{eV}=749.74 \AA$.
TABLE 29. $\mathrm{X}^{2} \Sigma^{+}\left(\mathrm{CO}^{+}\right) \leftarrow \mathrm{X}^{1} \Sigma^{+}$Rydberg series

\begin{tabular}{|c|c|c|c|c|}
\hline \multirow{2}{*}{$n$} & \multicolumn{2}{|c|}{$0-0$ series } & \multicolumn{2}{|c|}{$1-0$ series } \\
\hline & $\lambda$ & $\sigma$ & $\lambda$ & $\sigma$ \\
\hline & A & $\mathrm{cm}^{-1}$ & A & $c m^{-1}$ \\
\hline 6 & 938.3 & 106576 & 919.5 & 108761 \\
\hline 7 & 918.7 & 108849 & 900.7 & 111020 \\
\hline 8 & 908.4 & 110084 & 890.8 & 112265 \\
\hline 9 & 902.0 & 110865 & 884.8 & 113026 \\
\hline 10 & 898.0 & 111357 & 880.8 & 113538 \\
\hline 11 & 895. 2 & 111707 & 878.1 & 113882 \\
\hline 12 & 893.2 & 111957 & 876.2 & 114129 \\
\hline 13 & 891.7 & 112145 & 874.8 & 114312 \\
\hline 14 & 890.6 & 112284 & 873.8 & 114451 \\
\hline
\end{tabular}

Data from Takamine, Tanaka, and Iwata [253]. $\sigma_{\infty}(0-0)=113029 \mathrm{em}^{-1}=14.013 \mathrm{eV}=884.73 \AA( \pm 0.004 \mathrm{eV})$. $\sigma_{\infty}(1-0)=115190 \mathrm{em}^{-1}=14.281 \mathrm{eV}=868.13 \AA$.

TABLE 30. Band heads of the $\mathrm{B}^{2} \Sigma^{+} \rightarrow \mathrm{X}^{2} \Sigma^{+}$First Negative system of $\mathrm{CO}^{+}(R)$

\begin{tabular}{|c|c|c|c|c|c|c|c|}
\hline$\lambda$ & $\sigma$ & $I$ & $v^{\prime}-v^{\prime \prime}$ & $\lambda$ & $\sigma$ & $I$ & $v^{\prime}-v^{\prime \prime}$ \\
\hline$\AA$ & $\mathrm{cm}^{-1}$ & & & $\AA$ & $c m^{-1}$ & & \\
\hline 3152.71 & 31709.6 & 1 & $8-13$ & a 2325.17 & 42994.31 & 9 & 1-2 \\
\hline 3107.45 & 32171.4 & 2 & $7-12$ & a 2299.80 & 43468.68 & 10 & $0-1$ \\
\hline 3064.02 & 32627.4 & 3 & $6-11$ & 2298.18 & 43499.2 & 3 & $4-4$ \\
\hline 3022.97 & 33070.4 & 2 & $5-10$ & 2293.67 & 43584.8 & 1 & $10-8$ \\
\hline C 2984.16 & 33500.5 & 2 & $4-9$ & 2268.58 & 44066.8 & 3 & $3-3$ \\
\hline 2947.56 & 33916.5 & 1 & $3-8$ & 2255.75 & 44317.4 & 1 & $9-7$ \\
\hline 2938.54 & 34020.6 & 1 & $7-11$ & 2254.28 & 44346.3 & 2 & $6-5$ \\
\hline 2913. 18 & 34316.7 & 1 & $2-7$ & 2240.43 & 44620.4 & 4 & $2-2$ \\
\hline 2897.16 & 34506.4 & 3 & $6-10$ & 2222.66 & 44977.2 & 4 & $5-4$ \\
\hline 2882.23 & 34685.2 & 2 & $1-6$ & 2220.27 & 45025.6 & 0 & $8-6$ \\
\hline 2874.46 & 34778.9 & 0 & $9-12$ & 2214.46 & 45143.7 & 5 & $1-1$ \\
\hline 2858.08 & 34978.3 & 4 & $5-9$ & - 2189.85 & 45650.99 & 10 & $0-0$ \\
\hline 2820.82 & 35440.2 & 5 & $4-8$ & 2185.13 & 45749.6 & 4 & $7-5$ \\
\hline 2785.81 & 35885.6 & 5 & $3-7$ & 2164.34 & 46189.0 & 5 & $3-2$ \\
\hline в 2752.89 & 36314.67 & 6 & $2-6$ & 2154.15 & 46407.4 & 4 & $6-4$ \\
\hline 2745.10 & 36417.8 & 1 & $6-9$ & 2137.77 & 46763.0 & 6 & $2-1$ \\
\hline a 2722.32 & 36722.43 & 7 & $1-5$ & 2123.77 & 47071.2 & 3 & $5-3$ \\
\hline 2707.95 & 36917.4 & 3 & $5-8$ & 2112.38 & 47325.0 & 8 & $1-0$ \\
\hline в 2693.87 & 37110.35 & 2 & $0-4$ & 2095.27 & 47711.4 & 5 & $4-2$ \\
\hline в 2672.34 & 37409.27 & 7 & $4-7$ & 2090.95 & 47809.6 & 4 & $7-4$ \\
\hline b 2638.72 & 37885.9 & 8 & $3-6$ & 2067.92 & 48342.3 & 1 & $3-1$ \\
\hline a 2607.16 & 38344.46 & 8 & $2-5$ & 2067.81 & 48344.8 & 1 & $9-5$ \\
\hline B 2577.78 & 38781.52 & 10 & $1-4$ & 2061.03 & 48503.9 & 3 & $6-3$ \\
\hline a 2550.32 & 39198.99 & 7 & $0-3$ & 2042.29 & 48948.9 & 4 & $2-0$ \\
\hline 2534.79 & 39439.2 & 1 & $4-6$ & 2034.32 & 49140.6 & 2 & $8-4$ \\
\hline a 2504.47 & 39916.54 & 10 & $3-5$ & 2032.27 & 49190.3 & 1 & $5-2$ \\
\hline b 2474.21 & 40404.7 & 10 & $2-4$ & 2004.73 & 49865.9 & 0 & $4-1$ \\
\hline a 2445.82 & 40873.68 & 10 & $1-3$ & 2003.11 & 49906. 2 & 0 & $7-3$ \\
\hline a 2419.48 & 41318.69 & 8 & $0-2$ & 1983.92 & 50405.3 & 0 & $9-4$ \\
\hline 2412.43 & 41439.4 & 4 & $4-5$ & 1975.28 & 50625.7 & 0 & $6-2$ \\
\hline 2381.52 & 41977.1 & 5 & $3-4$ & 1831.05 & 54613.4 & 0 & $9-2$ \\
\hline 2362.46 & 42315.8 & 1 & $6-6$ & 1802.83 & 55468.4 & 0 & $8-1$ \\
\hline a 2352.56 & 42493.86 & 6 & $2-3$ & - & ......... & .... & - \\
\hline
\end{tabular}

Most of the band heads are taken from Biskainp [24].

a Data froll Rao [202a].

b Data from Sehmid [237]. Intensities are those of Biskamp [24].
- Listed by Biskamp as uneertain 8-12 or 4-9. Biskamp's measurements are probably good to $0.05 \AA$; Rao elaims aeeuraey to $1 \mathrm{~cm}^{-1}$. Johnson [118] has observed an additional band (unelassified) $2883.6 \AA \quad 34668.7 \mathrm{em}^{-1} \quad(I=2)$. 
TABLE 31. Band origins of the $\mathrm{B}^{2} \Sigma^{+} \rightarrow \mathrm{X}^{2} \Sigma^{+}$First Negative system of $\mathrm{CO}^{+}$

\begin{tabular}{|c|c|c|c|c|c|c|c|c|}
\hline \multirow{2}{*}{$v^{\prime}$} & \multicolumn{8}{|c|}{$v^{\prime \prime}$} \\
\hline & 0 & 1 & 2 & 3 & 4 & 5 & 6 & 7 \\
\hline 0 & 45633.44 & 43449.62 & 41295.97 & 39172. 76 & 37079.87 & & & \\
\hline 1 & - & 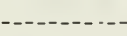 & 42975.40 & 40852.15 & 38759.31 & 36696.77 & & \\
\hline 2 & $-\cdots$ & & - & 42478.75 & 40385.72 & 38323. 19 & 36291.06 &.-- \\
\hline 3 & & & - & . & - n & 39898.85 & $(37866.7)$ & \\
\hline 4 & & & & & & & & 37392.19 \\
\hline
\end{tabular}

Data mostly from Rao [202a].

$(\quad)=$ Origin of 3-6 band from Coster, Brons, and Bulthuis [45].
Data of ref. [45] agree with that of ref. [202a] within $0.5 \mathrm{~cm}^{-1}$ Misprint in ref. [202a], 2-5 origin should be $38323.19 \mathrm{~cm}^{-1}$.

$\mathrm{T}_{\mathrm{ABLE}}$ 32. Band heads of the $\mathrm{A}^{2} \Pi_{i} \rightarrow \mathrm{X}^{2} \Sigma^{+}$Comet Tail system of $\mathrm{CO}^{+}(R)$

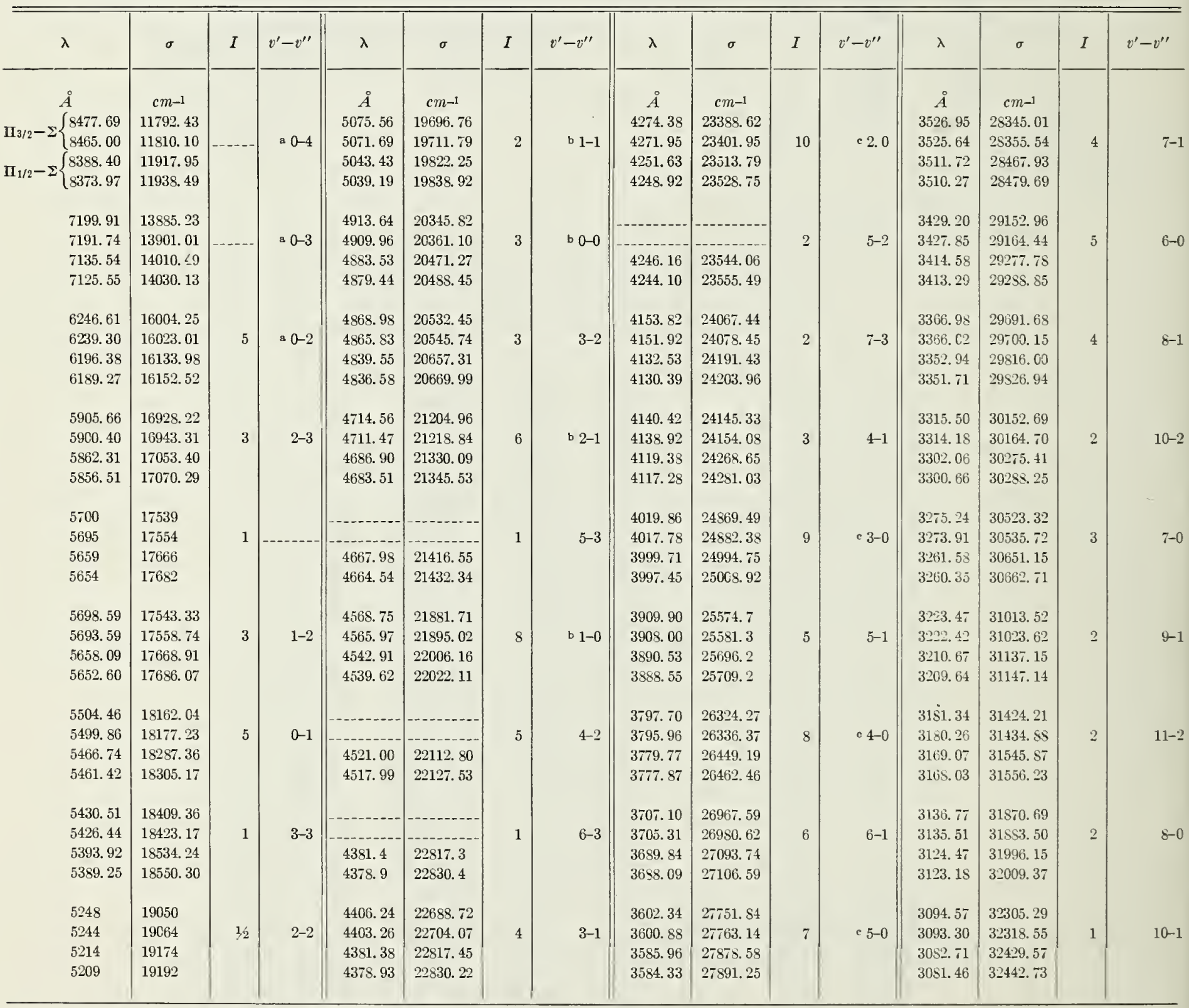

Data of Baldet [9] supplemented by data of (a) Rao [202b], (b) Schmid and Gerö [222], and (c) Coster, Brons, and Bulthuis [45]. Intensities are from Baldet [9]. Head-forming branches are $Q_{1}, R_{1}, R_{2}, R_{21}$, given in order of decreasing wavelength. Johnson [118] lists a double-headed band of intensity 2 (4139.0 $\AA$, $4123.2 \AA$ ) which may belong to this system. 
TABLE 33. Band origins of the $\mathrm{A}^{2} \mathrm{II}_{i}-\mathrm{X}^{2} \Sigma^{+}$Comet Tail system of $\mathrm{CO}^{+}$

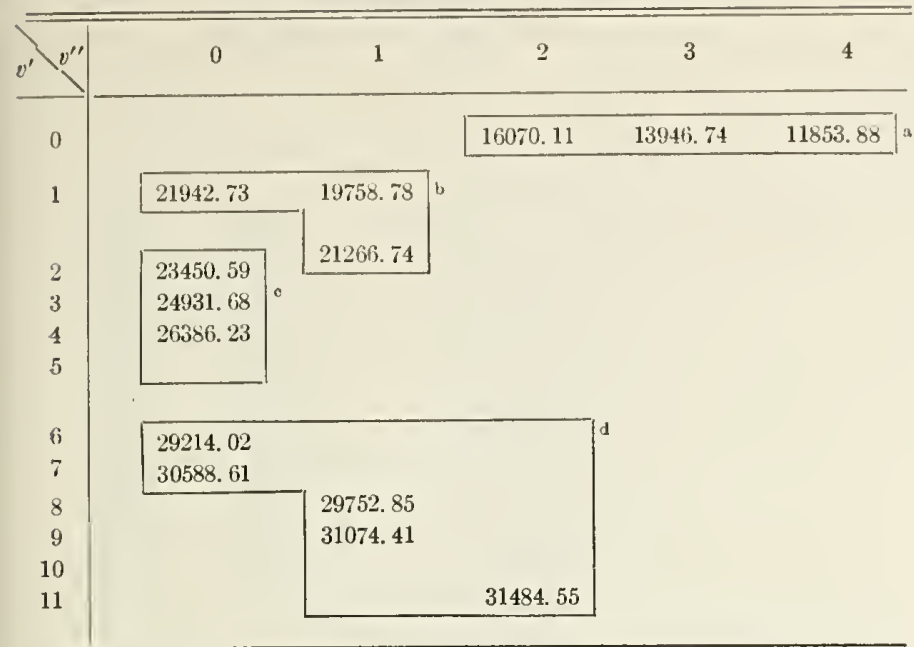

a Data from Rao [202b].

b Computed from the data of Schmid and Gerö [222].

c Computed from the data of Coster, Brons, and Bulthuis [45].

d Computed from the data of Bulthuis [34a].

Data compiled by Rao [202b].

TABLE 34. Band heads of the $\mathrm{B}^{2} \Sigma^{+} \rightarrow \mathrm{A}^{2} \Pi_{i}$ Baldet-Johnson system of $\mathrm{CO}^{+}(V)$

\begin{tabular}{|c|c|c|c|c|}
\hline$\lambda$ & $\sigma$ & $I$ & $v^{\prime}-v^{\prime \prime}$ & Ref \\
\hline$\AA$ & $\mathrm{em}^{-1}$ & & \multirow{5}{*}{$0-1$} & \multirow{5}{*}{$34 b$} \\
\hline 4236.28 & 23598.96 & 3 & & \\
\hline 4231.42 & 23626.09 & 9 & & \\
\hline 4212.98 & 23729.46 & 7 & & \\
\hline 4209.02 & 23751.82 & 8 & & \\
\hline 4201.5 & 23794 & 1 & \multirow{3}{*}{$1-2$} & \multirow{3}{*}{118} \\
\hline 4182.6 & 23902 & 1 & & \\
\hline 4179.1 & 23922 & 1 & & \\
\hline 3978.13 & 25130.36 & 4 & \multirow{4}{*}{$0-0$} & \multirow{4}{*}{$34 \mathrm{~b}$} \\
\hline 3973.31 & 25160.84 & 9 & & \\
\hline 3957.37 & 25262.13 & 6 & & \\
\hline 3953.52 & 25286.77 & 9 & & \\
\hline 3729.8 & 26803 & 3 & \multirow{4}{*}{$1-0$} & \multirow{4}{*}{118} \\
\hline 3725.0 & 26838 & 9 & & \\
\hline 3711.3 & 26937 & 9 & & \\
\hline 3707.6 & 26964 & 9 & & \\
\hline 3515.8 & 28435 & 2 & \multirow{4}{*}{$2-0$} & \multirow{4}{*}{118} \\
\hline 3511.7 & 28468 & 7 & & \\
\hline 3500.4 & 28560 & 3 & & \\
\hline 3496.7 & 28590 & 4 & & \\
\hline 3331.9 & 30004 & 1 & \multirow{4}{*}{$3-0$} & \multirow{4}{*}{118} \\
\hline 3329.0 & 30030 & 1 & & \\
\hline 3317.9 & 30131 & 1 & & \\
\hline 3314.8 & 30159 & 1 & & \\
\hline
\end{tabular}

Data from Johnson [118] and Bulthuis [34b]. M easurements of Baldet [8] differ from the above by up to $1 \AA$. Intensities are those given by Jonnson [118]. Ilead forming branehes are $\mathrm{P}_{1}, \mathrm{Q}_{1}, \mathrm{P}_{21}$, and $\mathrm{Q}_{21}$ in order of decreasing wavelength.
TABLE 35. Miscellaneous unclassified bands

(a) Band heads observed together with the $\mathrm{A}$ III $-\mathrm{X}^{1} \Sigma^{+}+$Fourth Positive systen (emission) b

\begin{tabular}{|c|c|c|c|}
\hline$\lambda$ & $\sigma$ & $I$ & Ref. \\
\hline$\AA$ & $c m^{-1}$ & & \\
\hline 2485.8 & 40216.4 &.- & 277 \\
\hline 2299.6 & 43472.4 & & 277 \\
\hline 2209.0 & 45255.2 & 1 & 59 \\
\hline 2098.19 & a 47644.9 & & 89 \\
\hline 2088.0 & 47877 &.-- & 59 \\
\hline 2064.0 & 48434 & $\ldots$ & 59 \\
\hline 2042.3 & 48949.5 & 1 & 90 \\
\hline 2040.0 & 49004 & & 59 \\
\hline 1953.0 & 51203.3 & 5 & I \\
\hline 1933.6 & 51717.0 & 2 & 23 \\
\hline 1837.2 & 54430.7 & 1 & L \\
\hline 1835.47 & 54481.9 & $\ldots$ & 89 \\
\hline 1827.6 & 54715.7 & 1 & 90 \\
\hline 1801.9 & 55497.0 & 2 & L \\
\hline 1772.9 & 56403.8 & 4 & 90 \\
\hline 1698.8 & 58865.1 & 1 & L. \\
\hline 1688.5 & 59224.2 & 1 & $\mathrm{~L}$ \\
\hline 1666.7 & 59998.8 & 1 & L \\
\hline 1492.6 & 66997.2 & 7 & 90 \\
\hline 1438.7 & 69507 & 1 & I. \\
\hline 1405.5 & 71149 & 1 & $\mathrm{~L}$ \\
\hline 1404.0 & 71225 & 1 & L \\
\hline 1386.4 & 72129 & 1 & L \\
\hline 1363.3 & 73350.4 & 1 & 90 \\
\hline 1343.0 & 74460 & 1 & L \\
\hline 1228.2 & 81422.6 & 1 & 90 \\
\hline 1152.9 & 86733.3 & 3 & 90 \\
\hline 1025.7 & 97496.3 & $\ldots$ & 90 \\
\hline
\end{tabular}

$\mathbf{L}=\mathrm{L}$ yman (see ref. 23 ).

a Original measurement was in vacuum. Air wavelengths listed above. b See see. 3.1.

(b) Band heads observed in absorption a

\begin{tabular}{|c|c|c|}
\hline$\lambda$ & $\sigma$ & $I$ \\
\hline$\AA$ & $c m^{-1}$ & \\
\hline 1569.5 & 63715 & 1 \\
\hline 1462.3 & 68385 & 3 \\
\hline 1433.3 & 69769 & 2 \\
\hline 1432.3 & 69818 & 2 \\
\hline 1422.4 & 70304 & 8 \\
\hline 1394.3 & $71 \quad 721$ & 1 \\
\hline 1369.5 & 73019 & 2 \\
\hline 1366.6 & $73 \quad 174$ & 8 \\
\hline 1352.4 & 73943 & 1 \\
\hline 1333.2 & 75008 & 1 \\
\hline 1329.3 & $75 \quad 228$ & 3 \\
\hline 1284.8 & 7833 & 1 \\
\hline 1218.6 & 82061 & 0 \\
\hline 1209.2 & 82699 & 0 \\
\hline 1181.8 & 84617 & 0 \\
\hline 1135.3 & s8 082 & 1 \\
\hline 1134.6 & 88137 & 1 \\
\hline 1130.4 & So 464 & 2 \\
\hline 1115.4 & 89654 & 1 \\
\hline 1115.1 & 59675 & 1 \\
\hline 1111.6 & s9 960 & 1 \\
\hline
\end{tabular}

Taken from Tanaka, Jursa, and Lel3hanc [255].

antensities of these are approximately $10^{3}$ times weaker than the strong A-X bands.

"The above bauds are probably due to intereombination bands of $\mathrm{CO}$. The strongest two bands appeared even at a pressure of $\sim 1 \mathrm{~mm} 11 \mathrm{~g} "$ [ 255$]$. 
TABLE 35. Miscellaneous unclassified bands-Continued

(c) Band heads observed with the $\mathbf{a}^{\prime 3 \Sigma^{+}} \rightarrow \mathrm{a}^{3} \Pi$ Asundi system

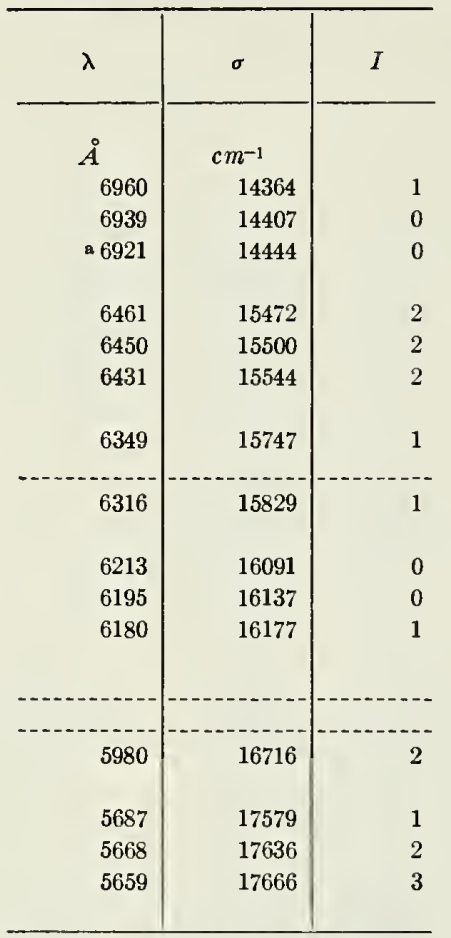

Data from McLennan, Smith, and Peters [158].

a Band head given as $6982 A$. It is assumed the correct wavenumber was given.

(d) Rand heads observed in emission by Tschulanowsky and Gassilewitsch

\begin{tabular}{|c|c|}
\hline$\lambda$ & $\sigma$ \\
\hline$\AA$ & $c m^{-1}$ \\
\hline 1183.62 & 84486.6 \\
\hline 1154.83 & 86592.8 \\
\hline 1123.63 & 88997.3 \\
\hline 1107.77 & 90271.4 \\
\hline 1083.24 & 92315.6 \\
\hline 1066.19 & 93791.9 \\
\hline 1057.60 & 94553.7 \\
\hline 1052.44 & 95017. 3 \\
\hline 1034.68 & 96648.2 \\
\hline 1032.78 & 96826.6 \\
\hline 1024. 70 & 97589.5 \\
\hline 1018.54 & 98179.7 \\
\hline 1011.78 & 98835.7 \\
\hline 1008.80 & 99127.7 \\
\hline 1007.77 & 99229.0 \\
\hline 1007.05 & 99299.9 \\
\hline 1005.26 & 99476.8 \\
\hline 983.84 & 101643 \\
\hline 980.65 & 101973 \\
\hline 962.47 & 103899 \\
\hline 961.27 & 104029 \\
\hline 952.45 & 104992 \\
\hline \& 948.42 & 105439 \\
\hline a 945.54 & 105760 \\
\hline 943.24 & 106017 \\
\hline 9 935.71 & 106871 \\
\hline
\end{tabular}

Data from Tschulanowsky and Gassilewitsch [261].

a Observed in absorption by Huffman, Larrabee, and Tanaka [107].
TABLE 35. Miscellaneous unclassified bands-Continued (e)

(e) Triplet bands observed in emission

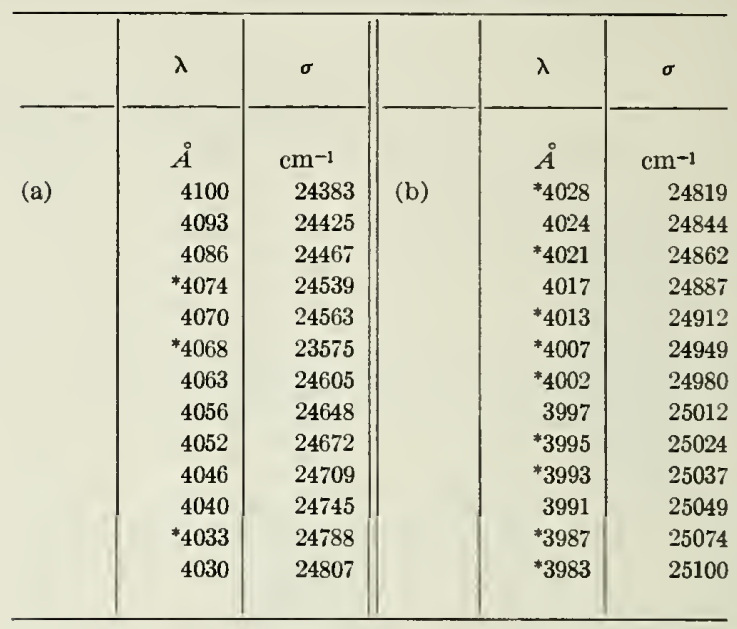

Data taken from Herman [95].

* Observed in comets; band positions less accurate than the others.

\begin{tabular}{|c|c|c|c|c|c|}
\hline$\lambda$ & $\sigma$ & $\lambda$ & $\sigma$ & $\lambda$ & $\sigma$ \\
\hline$\stackrel{\circ}{A}$ & $\mathrm{~cm}^{-1}$ & $\AA$ & $c m^{-1}$ & $\AA$ & $c m^{-1}$ \\
\hline $1,000.0$ & 100,000 & 910.8 & 109,790 & 788.7 & 126,790 \\
\hline 995.8 & 100,420 & 909.3 & 109,970 & 776.1 & 128,850 \\
\hline 990.9 & 100,920 & 908.5 & 110,070 & 771.2 & 129,670 \\
\hline 990.1 & 101,000 & 905.2 & 110,470 & 768.8 & 130,070 \\
\hline 989.4 & 101,070 & 903.7 & 110,660 & 760.5 & 131,490 \\
\hline 986.0 & 101,420 & 903.2 & 110,720 & 752.2 & 132,940 \\
\hline 985.5 & 101,470 & 900.1 & 111,100 & 749.2 & 133,480 \\
\hline 982.5 & 101,780 & 898.8 & 111,260 & 745.6 & 134,120 \\
\hline 977.5 & 102,300 & 895.4 & 111,680 & 737.3 & 135,630 \\
\hline 973.4 & 102,730 & 894.7 & 111,770 & 728.3 & 137,310 \\
\hline 972.9 & 102,785 & 891.8 & 112,130 & 722.6 & 138,390 \\
\hline 971.8 & 102,900 & 889.7 & 112,400 & 721.5 & 138,600 \\
\hline 970.5 & 103,040 & 889.1 & 112,470 & 719.2 & 139,040 \\
\hline 969.8 & 103,110 & 888.5 & 112,550 & 715.6 & 139,740 \\
\hline 968.9 & 103,210 & 888.1 & 112,600 & 714.5 & 139,960 \\
\hline 968.1 & 103,295 & 885.4 & 112,940 & 712.3 & 140,390 \\
\hline 964.6 & 103,670 & 867.9 & 115,220 & 707.8 & 141,280 \\
\hline 964.1 & 103,720 & 864.5 & 115,670 & 705.2 & 141,800 \\
\hline 960.4 & 104,120 & 863.8 & 115,770 & 702.1 & 142,430 \\
\hline 959.5 & 104,220 & 861.4 & 116,090 & 701.6 & 142,530 \\
\hline 956.3 & 104,570 & 859.7 & . 116,320 & 700.0 & 142,860 \\
\hline 954.1 & 104,810 & 858.3 & 116,510 & 698.8 & 143,100 \\
\hline 946.3 & 105,670 & 857.2 & 116,660 & 697.0 & 143,470 \\
\hline 941.2 & 106,250 & 854.5 & 117,030 & 695.2 & 143,840 \\
\hline 940.0 & 106,380 & 853.2 & 117,210 & 681.6 & 146,710 \\
\hline 933.2 & 107,160 & 852.4 & 117,320 & 680.6 & 146,930 \\
\hline 931.9 & 107,310 & 851.6 & 117,430 & 663.3 & 150,760 \\
\hline 930.1 & 107,515 & 849.4 & 117,730 & 650.0 & 153,850 \\
\hline 928.5 & 107,700 & 846.0 & 118,200 & 646.6 & 154,660 \\
\hline 925.9 & 108,000 & 841.4 & 118,850 & 634.4 & 157,630 \\
\hline 924.5 & 108,170 & 837.7 & 119,370 & 631.6 & 158,330 \\
\hline 922.6 & 108,390 & 834.5 & 119,830 & 630.6 & 158,580 \\
\hline 921.1 & 108,570 & 833.9 & 119,920 & 605.8 & 165,070 \\
\hline 919.6 & 108,740 & 832.5 & 120,120 & 604.8 & 165,340 \\
\hline 917.5 & 108,990 & 830.2 & 120,450 & 604.1 & 165,540 \\
\hline 915.7 & 109,210 & 821.4 & 121,740 & 601.9 & 166,140 \\
\hline 913.6 & 109,460 & 819.4 & 122,040 & & \\
\hline 912.2 & 109,630 & 795.6 & 125,690 & & \\
\hline
\end{tabular}

Meas. of Huffman et al., [107]. Good to $\pm 0.5 \AA$. 
TABLE 35. Miscellaneous unclassified bands-Continued Uncions

(g) Unclassified absorption bandsc

\begin{tabular}{|c|c|}
\hline$\lambda$ & $\sigma$ \\
\hline$\ddot{A}$ & $\mathrm{~cm}^{-1}$ \\
\hline 881.3 & 113470 \\
\hline $871.7^{b}$ & 114720 \\
\hline $848.6^{*}$ & 117840 \\
\hline $844.3^{*}$ & 118440 \\
\hline 839.3 & 119150 \\
\hline 836.1 & 119600 \\
\hline $827.2^{*}$ & 120890 \\
\hline 824.7 & 121260 \\
\hline 817.8 & 122280 \\
\hline 814.6 & 122760 \\
\hline $807.3^{*}$ & 123870 \\
\hline $804.8^{*}$ & 124250 \\
\hline 801.1 & 124830 \\
\hline 797.7 & 125360 \\
\hline $794.8^{*}$ & 125820 \\
\hline 791.6 & 126330 \\
\hline 789.7 & 126630 \\
\hline 786.3 & 127180 \\
\hline $783.1^{\mathrm{a}}$ & 127700 \\
\hline $779.5^{*}$ & 128290 \\
\hline $774.0^{*}$ & 129200 \\
\hline 765.8 & 130580 \\
\hline 757.2 & 132070 \\
\hline 750.4 & 133260 \\
\hline $734.2^{*}$ & 136200 \\
\hline 725.7 & 137800 \\
\hline
\end{tabular}

Data of Henning [91]

*These bands also seen in absorption by Huffman, Larrabee, and Tanaka [107].

- Observed also by Tanaka [254] as a closely spaced, triple-headed, and red degraded band.

b Seen in emission by Anand [2].

c See section 3.20.

(h) Emission and absorption bands of Anand

\begin{tabular}{|c|c|c|c|c|c|}
\hline & $\lambda$ & $\sigma$ & & $\lambda$ & $\sigma$ \\
\hline \multirow{8}{*}{ (a) } & $\dot{A}$ & $c m^{-1}$ & \multirow{7}{*}{ (b) } & $\dot{A}$ & $\mathrm{~cm}^{-1}$ \\
\hline & 906.2 & 110350 & & 897.7 & 111400 \\
\hline & 886.3 & 112830 & & 880.7 & 113550 \\
\hline & 875.7 & 114190 & & *871. 6 & 114730 \\
\hline & 869.4 & 115020 & & 866.5 & 115410 \\
\hline & 865.3 & 115570 & & & \\
\hline & 862.5 & 115940 & & & \\
\hline & \multicolumn{3}{|c|}{ Emission series } & \multicolumn{2}{|c|}{ Absorption series } \\
\hline
\end{tabular}

- Observed previously by Henning [91].

Data of Anand [2]; most of these bands have been observed recently, in absorption, by Huffman, Larrabee, and Tanaka [107]. See section 3.20.
TABLE 35. Miscellaneous unclassified bands-Continued

(i) Band heads observed together with the $\mathrm{A}^{2} \mathrm{II}_{\mathrm{r}} \rightarrow \mathrm{X}^{2} \Sigma+$ Comet Tail system of $\mathrm{CO}^{+}$

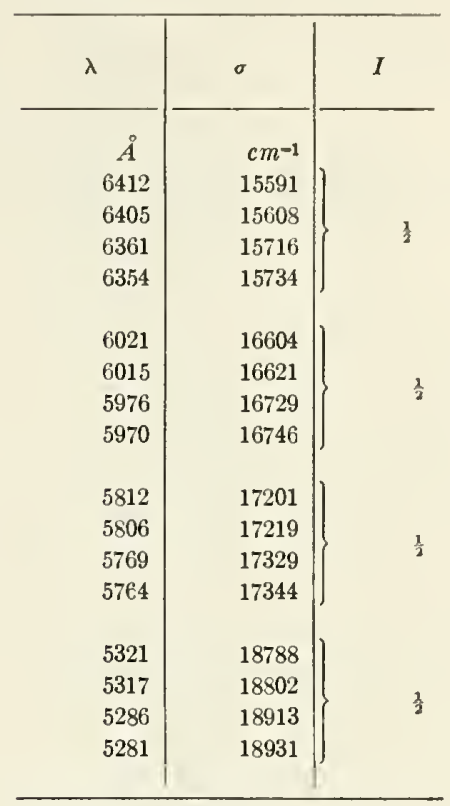

Data of Baldet [9]; bands are red-degraded.

(j) Band heads observed together with the $\mathrm{B}^{2} \Sigma^{+} \rightarrow \mathrm{A}^{2} \mathrm{II}_{i}$ system of $\mathrm{CO}^{+}$

\begin{tabular}{|c|c|c|}
\hline$\lambda$ & $\sigma$ & $I$ \\
\hline$\stackrel{\circ}{ }$ & $c m^{-1}$ & \\
\hline 3674.6 & 27206 & 3 \\
\hline 3662.6 & 27295 & 4 \\
\hline 3545.7 & 28195 & 1 \\
\hline 3534.1 & 28288 & 1 \\
\hline 3377.7 & 29597 & 5 \\
\hline 3370.3 & 29662 & 7 \\
\hline 3254.5 & 30718 & 4 \\
\hline 3247.5 & 30784 & 5 \\
\hline 3204.8 & 31194 & 0 \\
\hline 3199.6 & 31245 & 0 \\
\hline
\end{tabular}

Data from Johnson [118]. 
TABLE 36. Rotational constants for the $\mathrm{X}^{1} \Sigma^{+}$state

\begin{tabular}{|c|c|c|c|}
\hline Isotope & $v$ & $B_{v}$ & $\begin{array}{c}\mathrm{D} \text { 。 } \\
\left(10^{-8}\right)\end{array}$ \\
\hline $\mathrm{C}^{12} \mathrm{O}^{18}$ & $\begin{array}{r}0 \\
1 \\
2 \\
3 \\
4 \\
5 \\
6 \\
7 \\
8 \\
8 \\
10 \\
11 \\
12 \\
13 \\
14 \\
15 \\
16 \\
17 \\
18 \\
19 \\
20 \\
21 \\
22 \\
23 \\
24\end{array}$ & $\begin{array}{c}1.922521 \\
1.905014 \\
1.887513 \\
1.870010 \\
1.852513 \\
1.83514 \\
1.81766 \\
1.80018 \\
1.7829 \\
1.7829 \\
1.7475 \\
1.7296 \\
1.7130 \\
1.6957 \\
1.6778 \\
1.6599 \\
1.6434 \\
1.6256 \\
1.6090 \\
1.5910 \\
1.5732 \\
1.5561 \\
1.5390 \\
(1.5220) \\
1.5048\end{array}$ & $\begin{array}{l}6.1193 \\
6.1184 \\
6.1174 \\
6.1165 \\
6.1155\end{array}$ \\
\hline $\mathrm{C}^{13} \mathrm{O}^{16}$ & $\begin{array}{l}0 \\
1\end{array}$ & $\begin{array}{l}1.8380 \\
1.8216\end{array}$ & \\
\hline $\mathrm{C}^{12} \mathrm{O}^{18}$ & $\begin{array}{l}0 \\
1\end{array}$ & $\begin{array}{l}1.8319 \\
1.8156\end{array}$ & \\
\hline
\end{tabular}

(a) $v=0$ through 4, data from Rank et al. [198, 196] and Wiggins [273]. $\mathrm{H}_{0} \sim 5.8 \times 10^{-12} \mathrm{~cm}^{-1}[198,196]$. D values obtained from Dunham constants. $\mathrm{D}_{0}(\operatorname{exptl})=6.117 \times 10^{-6}$.

Uncer tainty in $\mathrm{B}_{0}$ is $\pm 0.0000035 \mathrm{~cm}^{-1}$.

(b) $v=5$ through 7 , data from Goldberg and Moller [85].

(c) $v=8$ through 24, data from Schmid and Gerö.[228].

(d) $v=23, \mathrm{~B}$ (calc).

(e) Data for $v=0$ through 7 derived from vibration-rotation spectra; data for $v \geq 8$ derived from $\mathrm{A}-\mathrm{X}$ system.

(f) $\mathrm{C}^{13} \mathrm{O}^{16}$ from Plyler, Blaine, and Tidwell [193].

(g) $\mathrm{C}^{12} \mathrm{O}^{18}$ from Mills and Thompson [162].
TABLE 37. Rotational constants for the $\mathrm{A}^{1} \Pi$ state

\begin{tabular}{l|r|r}
\hline \hline Isotope & \multicolumn{1}{|c|}{$v$} & \multicolumn{1}{c}{ B } \\
\hline & & \\
\hline $\mathrm{C}^{12} \mathrm{O}^{10}$ & 0 & 1.6001 \\
& 1 & 1.5775 \\
& 2 & 1.5561 \\
3 & 1.5329 \\
4 & 1.5089 \\
5 & 1.4861 \\
6 & 1.4616 \\
7 & 1.4384 \\
8 & 1.4138 \\
9 & 1.3900 \\
10 & 1.3651 \\
11 & 1.3421 \\
12 & 1.3167 \\
13 & 1.2915
\end{tabular}

Data from Schmid and Gerö [228]. These values obtained from $\mathrm{F}\left(\mathrm{J}+\frac{\mathrm{I}}{2}\right)$ Work currently in progress will provide more accurate values in the neal future [238a].

a Data from Onaka [182].

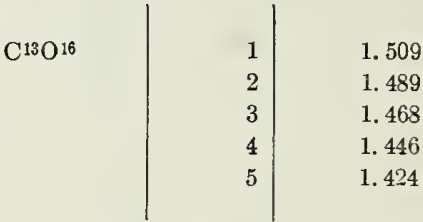

Data from McCulloh and Glockler [157b].

$\mathrm{T}_{\mathrm{ABLE}}$ 38. Rotational constants for the $\mathrm{B}^{1} \Sigma^{+}$state

\begin{tabular}{c|c|c|c}
\hline Isotope & $v$ & $\mathrm{~B} \cdot$ & $\begin{array}{c}\mathrm{D} \text { 。 } \\
\left(10^{-6}\right)\end{array}$ \\
\hline $\mathrm{C}^{12} \mathrm{O}^{16}-----$ & 0 & 1.9475 & $\sim 6.1$ \\
& 1 & 1.921 \\
& & \\
& & \\
& &
\end{tabular}

Data from Schmid and Gerö [224].

a Calculated; The identification of the $v=2$ level is uncertain [25s].

\begin{tabular}{l|l|l|r}
\hline $\mathrm{C}^{13} \mathrm{O}^{16}-\ldots$ & 0 & 1.863 & 5.4 \\
& 1 & 1.837 & $\ldots . . .-$. \\
\hline
\end{tabular}

Data from Douglas and M $\phi$ ller [56]. .

TABLE 39. Rolational constants for the $\mathrm{C}^{1} \Sigma^{+}$state

\begin{tabular}{|c|c|c|}
\hline$v$ & $B$, & $\begin{array}{c}\text { D. } \\
\left(10^{-6}\right)\end{array}$ \\
\hline 0 & \$1. 9436 & $b \sim 5.7$ \\
\hline 1 & a 1.924 & \\
\hline
\end{tabular}

- Data from ref. [276].

b Data from ref. [224]. 
TABLE 40. Rotational constants for the $\mathrm{E}^{\mathrm{I}} \mathrm{II}$ state

\begin{tabular}{l|r|r|r}
\hline \hline Isotope & $v$ & $\mathrm{~B}$, & $\begin{array}{c}\mathrm{D}, \\
\left(10^{-6}\right)\end{array}$ \\
\hline $\mathrm{C}^{12} \mathrm{O}^{16}$ & & & \\
$\mathrm{C}^{13} \mathrm{O}^{16} \ldots$ & 1.9644 & 6.50 \\
& a 0 & 1.8773 & 5.87 \\
\hline
\end{tabular}

Data from Tilford, Vanderslice, and Wilkinson [257].

a $\mathrm{T}_{\text {oo }}=92929.62 \mathrm{~cm}^{-1}$.

TABle 41. Rotational constants for the $\mathrm{a}^{\prime 3} \Sigma^{+}$state

\begin{tabular}{r|r}
\hline \hline$v$ & \multicolumn{1}{c}{ B } \\
\hline & \\
& \\
2 & 1.2995 \\
3 & 1.2823 \\
4 & 1.2658 \\
5 & 1.2478 \\
6 & 1.2307 \\
7 & 1.2140 \\
8 & 21.1950 \\
9 & 1.1816 \\
10 & 1.1655 \\
11 & $2(1.1470)$ \\
13 & 1.1176 \\
16 & 1.0692 \\
19 & 1.0206 \\
20 & 1.0037 \\
23 & 0.958 \\
&
\end{tabular}

Data from Herzberg and Hugo [101].

a Data from Gerö and Lörinczi [80].

TABLE 42. Rotational constants for the $a^{3} \Pi$ state

\begin{tabular}{|c|c|c|c|c|c|}
\hline$v$ & B。 & $\underset{\left(10^{-6}\right)}{D}$ & $\mathrm{Y}$ & A & Ref. \\
\hline 0 & 1.6803 & 6.1 & 24.7 & 41.5 & 32 \\
\hline 1 & 1.6627 & 6.4 & 25.0 & 41.5 & 32 \\
\hline 2 & 1.6433 & 6.4 & 25.1 & 41.2 & 32 \\
\hline 3 & 1.6231 & 6.2 & 25.3 & 41.0 & 32 \\
\hline 4 & 1. 6026 & 6.3 & & & 54,16 \\
\hline 5 & 1.5840 & 6.3 & & & 54,16 \\
\hline 6 & $\approx(1.5644)$ & & & & \\
\hline 7 & $\mathrm{~A}(1.5449)$ & & & & \\
\hline
\end{tabular}

Data from Buđó [32], Dieke and Mauchly [54], and Beer [16]. a Calculated.

$A($ average $)=41.3$.

TABLE 43. Rotational constants for the $\mathrm{b}^{3} \Sigma^{+}$state

\begin{tabular}{|c|c|}
\hline$v$ & $\mathrm{~B}$. \\
\hline 0 & 1.965 \\
\hline 1 & 1.923 \\
\hline
\end{tabular}

Data from Stcpanov [246]. Schmid and Gerö $[72,226]$ list $B_{0}=2.058, B_{1}=$ 2.025; but see [246] and footnote $b^{3} \Sigma+$ to table 1.
TABLE 44. Rotational constants for the $\mathrm{c}^{3} \mathrm{\Sigma}^{+}$state

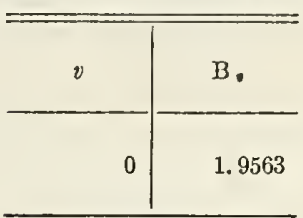

Data from Gerö [77].

TABLE 45. Rotational constants for the $\mathrm{d}^{3} \Delta_{i}$ state

\begin{tabular}{r|r|r|l}
\hline \hline$v$ & \multicolumn{1}{|c|}{ B } & $\begin{array}{r}\mathrm{D} \cdot \\
\left(10^{-6}\right)\end{array}$ & \multicolumn{1}{|c}{$\mathrm{A}$} \\
\hline & & & \\
\hline 3 & 1.2512 & 8.2 & -16.17 \\
4 & 1.2344 & 9.0 & -16.63 \\
7 & 1.1840 & 11.6 & -16.9 \\
9 & 1.1506 & 10.1 & -17.1 \\
\hline
\end{tabular}

Data from Carroll [40].

TABLE 46. Rotational constants for the $\mathrm{e}^{3} \Sigma^{-}$state

\begin{tabular}{r|c}
\hline \multicolumn{1}{c|}{$v$} & B 0 \\
\hline 2 & 1.2399 \\
3 & 1.2221 \\
4 & 1.2048 \\
6 & 1.1720 \\
8 & 1.1360 \\
10 & 1.1052 \\
\hline
\end{tabular}

Data from Herzberg and Hugo [101] with $v^{\prime}$ numbering one unit larger than in that reference [238a].

TABLE 47. Rotational constants for the $E_{0}{ }^{1} \Sigma^{+}$state

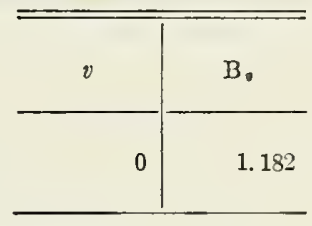

Data from Tschulanovsky [260].

TABLE 48. Rotational constants for the ${ }^{1} \mathrm{II}$ state

\begin{tabular}{|c|c|}
\hline 0 & B. \\
\hline 0 & 1.139 \\
\hline
\end{tabular}

Data from Tschulanovsky [260].

TABLE 49. Rotational constants for the $\mathrm{f}^{3} \Sigma^{+}$state

\begin{tabular}{rr|r}
\hline \multicolumn{1}{c|}{} & B, \\
\hline & & 0.83 \\
$?$ & 0.75 \\
\end{tabular}

Data [rom Gerö [78].

a $y=0$ is assumed, but uncertain.

Stcpanov [247] obtained $\mathrm{B}_{0}=0.800, \mathrm{~B}_{2}=0.711$.

Both sets of values are uncertain. 
TABLE 50. Rotational constants for the $\mathrm{X}^{2} \Sigma^{+}$state of $\mathrm{CO}^{+}$

\begin{tabular}{c|c|c}
\hline \hline \multicolumn{1}{c|}{} & $\mathrm{B}$, & $\begin{array}{c}\mathrm{D}, \\
\left(10^{-6}\right)\end{array}$ \\
\hline & & \\
& & \\
0 & 1.96773 & $(6.36)$ \\
1 & 1.94871 & $(6.37)$ \\
2 & 1.92960 & $(6.38)$ \\
3 & 1.91039 & 6.39 \\
4 & 1.89107 & $(6.40)$ \\
5 & 1.87178 & $(6.41)$ \\
6 & 1.85244 & $(6.42)$ \\
7 & 1.8328 & $(6.43)$ \\
\hline
\end{tabular}

Data from Rao [202a]. All D. values are estimated, except for $v=3$.

TABLE 51. Rotational constants for the $\mathrm{A}^{2} \Pi_{i}$ state of $\mathrm{CO}^{+}$

\begin{tabular}{|c|c|}
\hline 0 & B. \\
\hline 0 & 1.57971 \\
\hline 1 & 1.56022 \\
\hline 2 & 1.54070 \\
\hline 3 & 1. 52152 \\
\hline 4 & 1.50203 \\
\hline 5 & $\mathrm{a}(1.48259)$ \\
\hline 6 & 1.46319 \\
\hline 7 & 1.44375 \\
\hline 8 & 1. 42409 \\
\hline 9 & 1. 40509 \\
\hline 10 & $\mathrm{a}(1.38549)$ \\
\hline 11 & 1. 36515 \\
\hline
\end{tabular}

Data from Rao [202b]. D is estimated as $\sim 6.60 \times 10^{-6} ; A=-117.5$. - Calculated.
TABLE 52. Rotational constants for the $\mathrm{B}^{2} \Sigma^{+}$state of $\mathrm{CO}^{+}$

\begin{tabular}{c|c|c}
\hline \hline \multirow{2}{*}{$v$} & B. & $\begin{array}{c}\mathrm{D} . \\
\left(10^{-6}\right)\end{array}$ \\
\hline & & \\
\hline 0 & 1.78480 & 7.89 \\
1 & 1.75460 & 8.06 \\
2 & 1.72424 & 8.27 \\
3 & 1.69409 & 8.56 \\
4 & 1.6636 & 8.5 \\
\hline
\end{tabular}

Data from Rao [202a].

$\mathrm{T}_{\mathrm{ABLE}}$ 53. Doublet splitting constants for states of $\mathrm{CO}^{+}$

\begin{tabular}{|c|c|c|c|c|}
\hline \multicolumn{2}{|c|}{ B-X transition } & \multirow{2}{*}{0} & \multicolumn{2}{|c|}{$\gamma$} \\
\hline$v^{\prime}-v^{\prime \prime}$ & $\left|\gamma^{\prime}-\gamma^{\prime \prime}\right|$ & & $\left(\mathrm{X}^{2} \Sigma^{+}\right)$ & $\left(\mathrm{B}^{2} \Sigma+\right)$ \\
\hline $0-1$ & 0.0093 & 0 & & 0.0192 \\
\hline $0-2$ & .0081 & 1 & 0.0285 & \\
\hline $0-3$ & .0105 & 2 & .0273 & \\
\hline $1-2$ & .0098 & 3 & .0297 & \\
\hline $1-3$ & .0084 & & & \\
\hline $1-4$ & .0100 & & & \\
\hline $1-5$ & . $\mathrm{C093}$ & & & \\
\hline $2-4$ & .0090 & & & \\
\hline $2-5$ & .0090 & & & \\
\hline $2-6$ & .0088 & & & \\
\hline $3-5$ & .0079 & & & \\
\hline
\end{tabular}

Data on $\left|\gamma^{\prime}-\gamma^{\prime \prime}\right|$ from Rao [202a].

Data on $\gamma$ from Rao and Sarma [205].

TABLE 54. Perturbations of the electronic states of $\mathrm{CO}$ and $\mathrm{CO}^{+}$

\begin{tabular}{|c|c|c|c|c|c|}
\hline \multicolumn{6}{|c|}{ (a) A'II state } \\
\hline$v$ & $\begin{array}{c}\text { Max. pert. } \\
\text { (J) }\end{array}$ & $\begin{array}{c}\text { Perturbing } \\
\text { term }\end{array}$ & $\begin{array}{c}\text { Perturber } \\
\text { B. }\end{array}$ & Ref. & Comments \\
\hline 0 & $\begin{array}{l}\sim 1 \\
8,10,17 \\
9,12,16 \\
25 \\
27,31,34-5 \\
28-9 \\
>30\end{array}$ & $\begin{array}{l}\left.\text { in (or }{ }^{1} \Delta\right) \\
e^{3} \Sigma^{-}, v=1 \\
d^{3} \Delta_{i}, v=4 \\
\prod^{3} \text { or }{ }^{3} \Delta_{i} \\
\text { 3I }\end{array}$ & $\begin{array}{c}\mathrm{cm}^{-1} \\
>1.6 \\
1.247-1.254\end{array}$ & $\begin{array}{l}44,71 \\
268-9 \text { b } \\
216,44,225,262 \\
50 \\
44,216 \\
268,216 \\
44,71\end{array}$ & $\begin{array}{l}\text { See fig. } 1 \text { of ref. [44]a. Reality of perturbation disputed [140]. } \\
\text { Extra lines J=11 to } 14 ; \mathrm{B}-\mathrm{A}, 0-0 \text { band [216]. } \\
\mathrm{Q} \text { branch line magnetically sensitive. } \\
\text { Schmid and Gerö [225] called pertuber }{ }^{3} \Pi \text { at } 65002 \mathrm{~cm}^{-1}, \mathrm{~B}, \sim 1.37 \text {. } \\
\text { Not singlet [268]. Only Q branch perturbed. }\end{array}$ \\
\hline
\end{tabular}

- Both $\Lambda$-components are affected; $1 \Delta$ perturber is a possibility. The reported perturbation is large at J 16

- Extra lines [269] for B-A, o-o band at P(8), P(17), Q(8), Q(10), Q(17).

- Extra lines P, R(9), P, R(16), Q(11),(12),(13),(14) [216, 44].

1

$\mid \begin{aligned} & \sim 1 \\ & \sim 1 \\ & 8 \\ & 23-4,26,29 \\ & 25-6 \text { b } \\ & \\ & 26 \\ & 26 \\ & 28 \\ & 29 \text { b } \\ & 34-5 \\ & >35 \\ & 44\end{aligned}$

\begin{tabular}{|c|c|c|}
\hline in & -.. & $225,71,216$ \\
\hline${ }^{3} \Pi\left(\right.$ or $\left.{ }^{3} \Delta\right)$ &.-- & 216 \\
\hline $3 \Sigma$ & 1.155 & 262,269 \\
\hline$a^{\prime}{ }^{3+} \Sigma^{+}, v=9$ & $1.15-1.18$ & $\begin{array}{l}216,44,43 \\
262\end{array}$ \\
\hline $1 \Sigma^{-}$ & 1.50 & $\begin{array}{l}262,44 \\
225,74\end{array}$ \\
\hline $1 \Sigma^{+}$ & & 43,209 \\
\hline $3 \Sigma$ & $---\cdot$ & 44 \\
\hline & $-\cdots+$ & 44 \\
\hline & $-\cdots$ & 43 \\
\hline$a^{3} \Pi$ & 1.46 & $\begin{array}{l}225,45 \\
216\end{array}$ \\
\hline$\Sigma^{-}$ & - & 74 \\
\hline
\end{tabular}

All branches crossed at the same J. Perturber not $\Sigma$.

$\mathrm{Q}$ branch, $\mathrm{A}-\mathrm{X}$ band. Perturber probably $a^{\prime} \Sigma^{+}$.

Perturbing term estimated at $66499 \mathrm{~cm}^{-1} ; a^{\prime 3} \Sigma^{+}, v=9$ is at $66575 \mathrm{~cm}^{-1}$.

Ref. [43] estimates perturber $B$ o to be $>1.59$.

[44] lists this perturbation, Gerö [74] identified the perturber. The reality of this perturbation is not established. It is overlapped by a ${ }^{3} \Sigma+$ perturbation. [262] believes this to be a ${ }^{3} \Sigma$ perturbation with $\mathrm{B}, \sim 1.155$.

$\mathrm{Q}$ branch; see also ref. [52].

$\mathrm{P}, \mathrm{R}$ branch; extra line $\mathrm{R}-\mathrm{X}^{\mathrm{a}}=5.63 \mathrm{~cm}^{-1}$.

P branch.

$\mathrm{Q}$ branch; extra line, $\mathrm{R}-\mathrm{X}=-4.07 \mathrm{~cm}^{-1}$.

$\mathrm{Q}$ branch; A-X. See also $[262,44,52,234 \mathrm{~b}]$.

$P, R$ branches.

a $\mathrm{R}-\mathrm{X}=$ regular-extra.

b Extra lines R(26), Q(29), B-A [43,44]; P (26), Q(29), A-X [262]. 
TABLE 54. Perturbations of the electronic states of $\mathrm{CO}$ and $\mathrm{CO}^{+}-$Continued

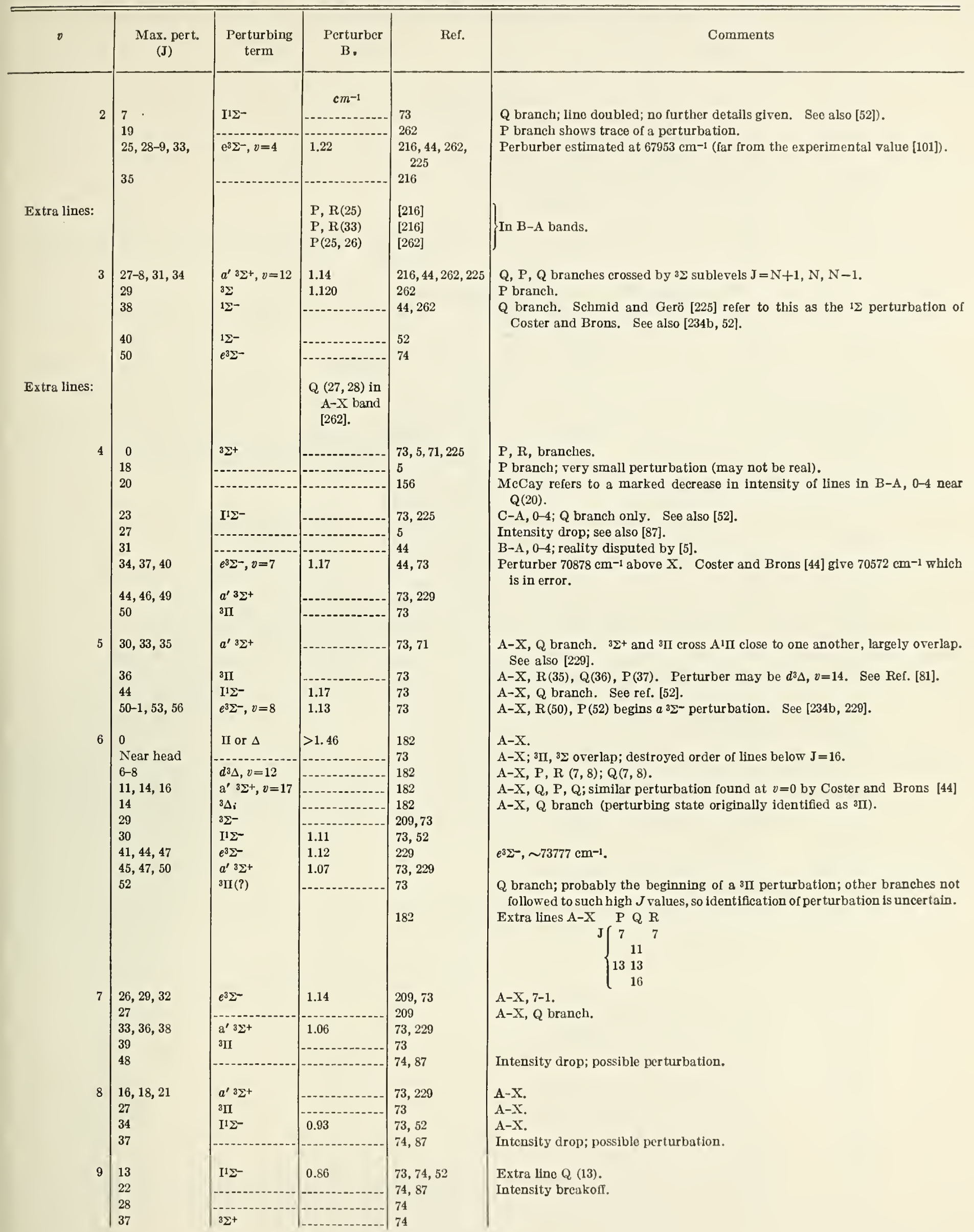


TABle 54. Perturbations of the electronic states of $\mathrm{CO}$ and $\mathrm{CO}^{+}-$Continued

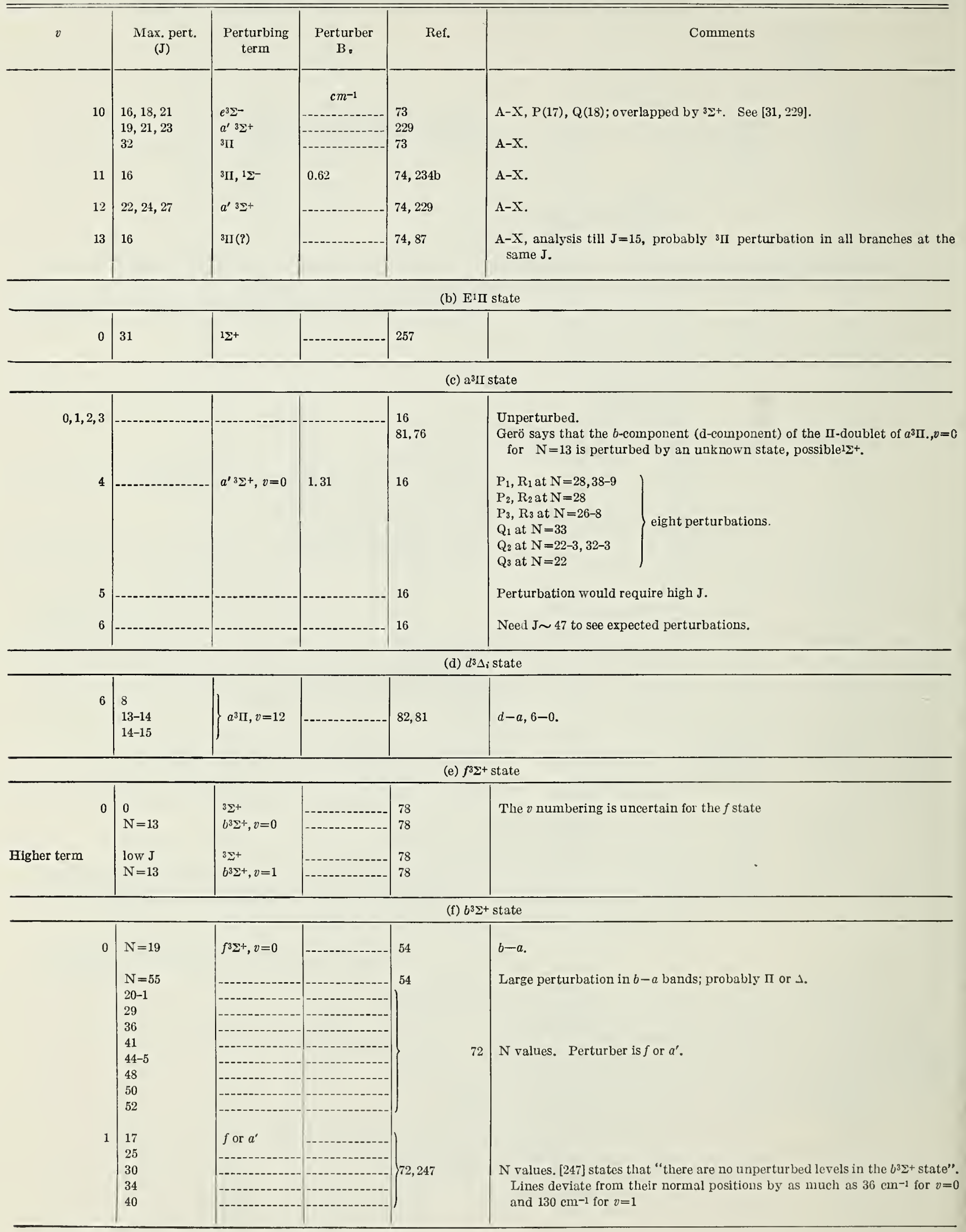


TABLe 54. Perturbations of the electronic states of $\mathrm{CO}$ and $\mathrm{CO}^{+}-$Continued

\begin{tabular}{l|c|c|c|c|c}
\hline \hline \multirow{2}{*}{$\begin{array}{c}\text { Max }) \\
\text { (J) pert. }\end{array}$} & $\begin{array}{c}\text { Perturbing } \\
\text { term }\end{array}$ & $\begin{array}{c}\text { Pcrturber } \\
\text { B. }\end{array}$ & Ref. & Comments \\
\hline
\end{tabular}

(g) A ${ }^{2} \mathrm{II}_{i}$ statc of $\mathrm{CO}^{+}$

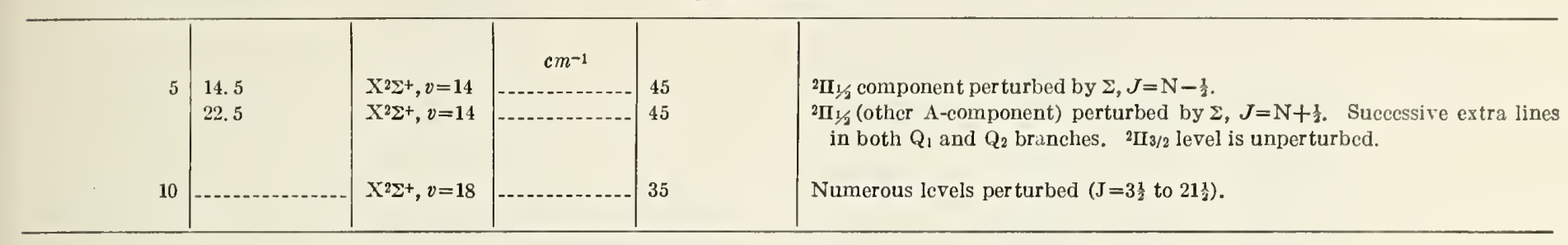

TaBle 55. Observed predissociations in $\mathrm{C}^{12} \mathrm{O}^{16}$ and $\mathrm{C}^{13} \mathrm{O}^{10}$ a

\begin{tabular}{|c|c|c|c|c|c|c|c|}
\hline Molecule & Term & $v$ & $\begin{array}{l}\mathrm{J} \text { for last } \\
\text { strong line }\end{array}$ & $\begin{array}{l}J \text { for first } \\
\text { weak line }\end{array}$ & $\begin{array}{c}\text { Rotational } \\
\text { energy }\end{array}$ & $\begin{array}{c}\begin{array}{c}\text { Energy } \\
\text { above } X, \\
v=0, J=0\end{array}\end{array}$ & Ref. \\
\hline \multirow[t]{6}{*}{$\mathrm{C}^{12} \mathrm{O}^{16}$} & $\mathrm{~B} 1 \Sigma^{+}$ & 0 & 37 & 38 & $\begin{array}{l}2738.2 \\
2886.2\end{array}$ & $\begin{array}{l}89655.9 \\
89803.9\end{array}$ & 56,43 . \\
\hline & & 1 & 17 & 18 & $\begin{array}{l}537.8 \\
657.0\end{array}$ & $\begin{array}{l}89587.6 \\
89656.8\end{array}$ & $56,224,74$. \\
\hline & $b^{3 \Sigma^{+}}$ & 0 & 55 & 56 & $\begin{array}{l}6052.2 \\
6273.3\end{array}$ & $\begin{array}{l}89884.7 \\
90104.8\end{array}$ & $74,72,226,75,30$. \\
\hline & & 1 & 42 & 43 & $\begin{array}{l}3472.9 \\
3638.3\end{array}$ & $\begin{array}{l}89488 \\
89653\end{array}$ & 74. \\
\hline & $\mathrm{C}^{1} \mathrm{\Sigma}^{+}$ & 0 & 28 & $\begin{array}{r}29 \\
29\end{array}$ & $\begin{array}{l}1577.1 \\
1689.7\end{array}$ & $\begin{array}{l}93497.8 \\
93610.4\end{array}$ & $74,224 \mathrm{~b} \quad 221$ \\
\hline & $\mathrm{c}^{3} 2^{+}$ & 0 & $-\cdots$ & $\sim 27$ & $(1479.0)$ & & 77,221 \\
\hline \multirow[t]{2}{*}{$\mathrm{C}^{13} \mathrm{O}^{16}$} & $\mathrm{~B}^{1} \Sigma^{+}$ & 0 & 39 & 40 & $\begin{array}{l}2893.1 \\
3040.8\end{array}$ & $\begin{array}{l}89808.4 \\
89956.1\end{array}$ & 56 (Using observed ZPE from ref. $157 \mathrm{~b}$ ). \\
\hline & & 1 & 19 & 20 & $\begin{array}{l}697.7 \\
771.0\end{array}$ & $\begin{array}{l}89650.3 \\
89723.6\end{array}$ & \\
\hline
\end{tabular}

- Hagstrum [87] bas summarized the predissociations using slightly different data. 
$\mathrm{T}_{\mathrm{ABLE}}$ 56. Vibration-rotation bands of $\mathrm{CO}$ in the infrared region

\begin{tabular}{|c|c|c|c|c|}
\hline \multirow{2}{*}{ Isotope } & \multicolumn{2}{|c|}{ Origin $\sigma_{0}$} & \multirow{2}{*}{ Band } & \multirow{2}{*}{ Ref. } \\
\hline & Obs. & Calc. ${ }^{b}$ & & \\
\hline & $c n$ & & & \\
\hline \multirow[t]{10}{*}{$\mathrm{C}^{12} \mathrm{O}^{10}$} & 2116.80 & - & $2-1$ & 193 \\
\hline & 2143.274 & 0.2731 & $1-0$ & 192,193 \\
\hline & 3996.88 & .9466 & $7-5$ & 85 \\
\hline & 4049.24 & .2958 & $6-4$ & 85 \\
\hline & 4101.73 & .7820 & $5-3$ & 85 \\
\hline & 4154.404 & .4056 & $4-2$ & 273 \\
\hline & 4207.168 & .1664 & $3-1$ & 273 \\
\hline & 4260.064 & .0646 & $2-0$ & a 197 \\
\hline & 6350.436 & .4404 & $3-0$ & 197 \\
\hline & 8414.458 & .4702 & $4-0$ & 102 \\
\hline $\mathrm{C}^{13} \mathrm{O}^{18}$ & 2096. 071 & & $1-0$ & 193 \\
\hline $\mathrm{C}^{12} \mathrm{O}^{18}$ & 2092. 12 & & $1-0$ & 162 \\
\hline
\end{tabular}

a Absolute accuracy claimed is 1 part in $5 \times 10^{6}$

Relative accuracy claimed is 1 part in $10^{7}$ measurement

Calculated line frequencies accurate to 1 part in $10^{\circ}$

Similar accuracies claimed for 1-0 band [196].

b Calculated origins from Wiggins [273]; some data previously given by Rank et al., [198].
TABLE 57. Dipole moments for states of $\mathrm{CO}$ and $\mathrm{CO}^{+}$

\begin{tabular}{l|c|c}
\hline \hline Molecule & State & $\mu$ (Debye) \\
\hline $\mathrm{CO}$ & $\mathrm{X} 1 \Sigma$ & $\mathrm{s} 0.114 \pm 0.005$ \\
& $\mathrm{~A} 1 \mathrm{03}$ & $.15 \pm 0.08$ \\
& $\mathrm{~B}^{1 \Sigma}$ & $\mathrm{b} 1.38$ \\
$\mathrm{a}^{3} \amalg$ & $.1 \pm 0.1$ \\
$\mathrm{CO}^{+}$ & $\mathrm{X}^{2 \Sigma}$ & $.1 \pm 0.1$ \\
& $\mathrm{~A}^{2} \Pi$ & \\
\hline
\end{tabular}

a This is the value of Burrus [36] to which has been added a polarizability correction of $0.002 \mathrm{D}[163]$ ( $\left.1 \mathrm{D}=10^{-18} \mathrm{esu}\right)$.

b A preliminary value determined from the radio frequency spectrum of the $a^{3}$ II state [67(a)].

Data from [134].

TABLE 58. Rotational frequencies, rotational constants, and magnetic moments of carbon monoxide

\begin{tabular}{|c|c|c|c|c|c|c|c|}
\hline Isotope & Frequency $\nu$ & $\begin{array}{c}\text { Transition } \\
J^{\prime} \leftarrow J^{\prime \prime}\end{array}$ & $\begin{array}{l}\text { Wave } \\
\text { length }\end{array}$ & $\mathrm{B} .0^{\circ}$ & $B e^{d}$ & $\mu \mathrm{J}(=g)^{\mathrm{a}}$ & Ref. \\
\hline \multirow{6}{*}{$\mathrm{C}^{12} \mathrm{O}^{16}$} & $M H z$ & & $m m$ & $M H z$ & $M H z$ & $\begin{array}{c}\text { Nuclear } \\
\text { magnetons }\end{array}$ & \multirow{12}{*}{215,47} \\
\hline & $691472.60 \pm 0.60$ & $6 \leftarrow 5$ & 0.43 & & & o n & \\
\hline & $576267.75 \pm 0.10$ & $5 \leftarrow 4$ & 0.52 & & & & \\
\hline & $461040.68 \pm 0.06$ & $4 \leftarrow 3$ & 0.65 & 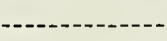 & & & \\
\hline & $345795.900 \pm 0.090$ & $3 \leftarrow 2$ & 0.87 & $\ldots . .$. & & $\cdots$ & \\
\hline & $230537.974 \pm 0.030$ & $2 \leftarrow 1$ & 1.30 & & & & \\
\hline & $115271.204 \pm 0.005$ & $1 \leftarrow 0$ & 2.60 & e 57898.462 & 57907.863 & -0.26910 & \\
\hline $\mathrm{C}^{12} \mathrm{O}^{17}$ & b $112359.276 \pm 0.060$ & $1 \leftarrow 0$ & -....- & 56432.560 & 56441.491 & -.26227 & \\
\hline $\mathrm{C}^{13} \mathrm{O}^{16}$ & $110201.370 \pm 0.008$ & $1 \leftarrow 0$ & $-\ldots$ & 55346.453 & 55355.038 & -.25704 & \\
\hline $\mathrm{C}^{12} \mathrm{O}^{18}$ & $109782.182 \pm 0.008$ & $1 \leftarrow 0$ & -- & 55135.337 & 55143.861 & -.25622 & \\
\hline $\mathrm{C}^{14} \mathrm{O}^{16}$ & $105871.110 \pm 0.004$ & $1 \leftarrow 0$ & & 53166.929 & 53174.841 & $\mathrm{~b}-.24664$ & \\
\hline $\mathrm{C}^{13} \mathrm{O}^{18}$ & $104711.416 \pm 0.008$ & $1 \leftarrow 0$ & - & 52583.285 & 52591.033 & $\mathrm{~b}-.24418$ & \\
\hline
\end{tabular}

a The error of $\pm 0.0005 \mathrm{MHz}$ to these values is the absoluteerror; the relative errors are about three times smaller.

b Calculated.

- Uncorrected values derived from frequency measurement with values of $\alpha_{\ominus}=525.03 \mathrm{MHz}, \gamma_{e}=0.0887 \mathrm{MHz}, \mathrm{D}_{e}=0.18347 \mathrm{MHz}$ [17]. These $\mathrm{B}_{\theta}$ values differ slightly from those given in ref. [215] primarily because of the different value of $\alpha_{\theta}$, which still introduces an uncertainty in the $B_{0}$ values of about $0.2 \mathrm{MHz}$. d Corrected for nonspherical distribution of the electrons, wobble stretching, and the Dunham corrections $[215,47]$.

- $\mathrm{B}_{0}=57635.970 \pm 0.003 \mathrm{MHz} ; \mathrm{D}_{0}=0.18390 \pm 0.00014 \mathrm{MHz}$ [122, 86]. This value of $\mathrm{D}_{0}$ is not compatible with the preferred value of $0.18345 \mathrm{MHz}$ derived from IR data $[198,196]$. 

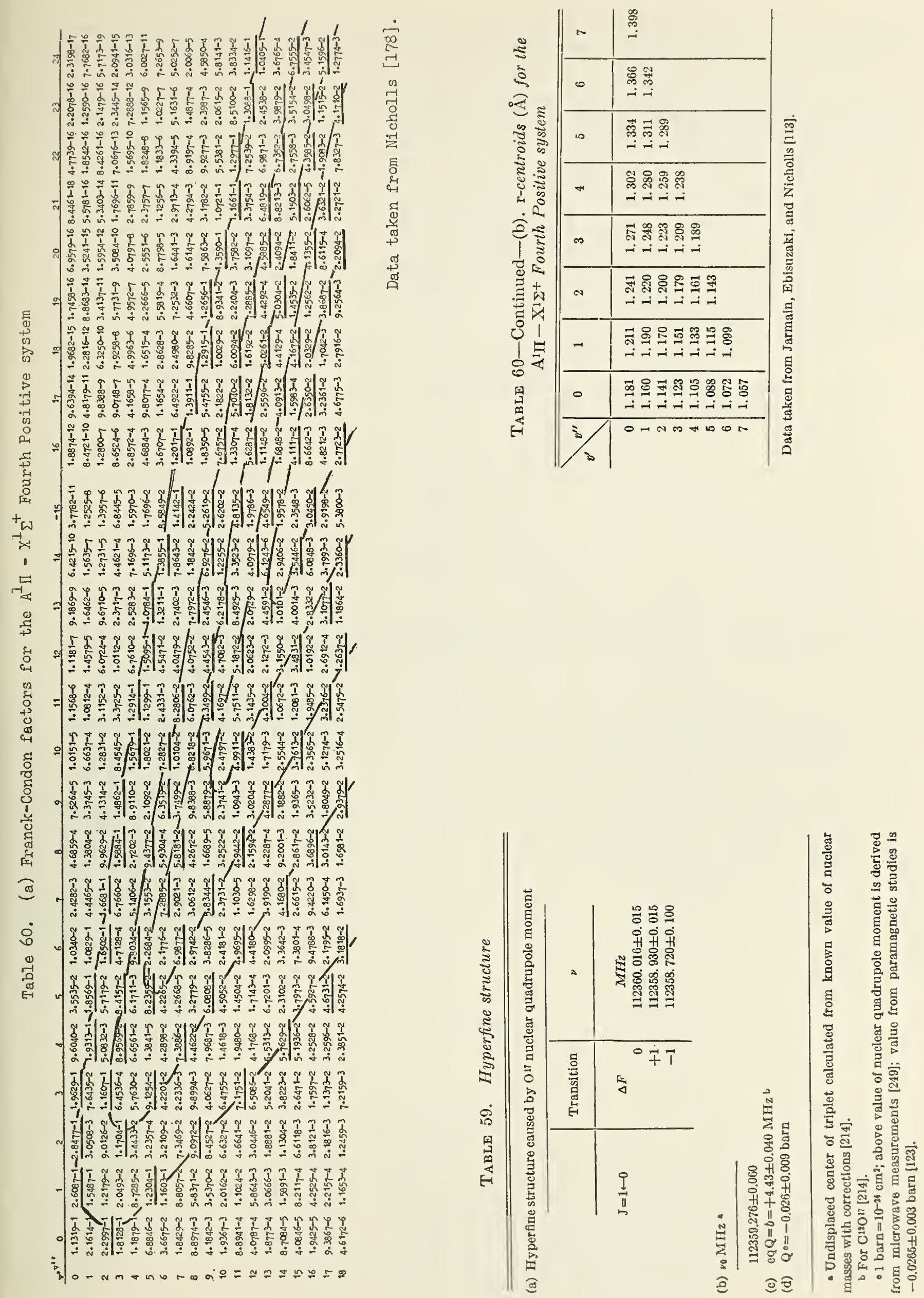
TABLE 61. Franck-Condon factors, band strengths, r-centroids, and $\mathrm{I}_{\infty}$ for the $\mathrm{B}^{1} \Sigma^{+}-\mathrm{A}^{1} \Pi$ A Angstrom system

\begin{tabular}{|c|c|c|c|c|c|c|c|c|c|c|c|c|c|c|}
\hline & 0 & 1 & 2 & 3 & 4 & 5 & 6 & 7 & 8 & 9 & 10 & 11 & 12 & 13 \\
\hline 0 & $\begin{array}{l}0.08898 \\
0.088 \\
1.177 \\
6.8\end{array}$ & $\begin{array}{c}0.18159 \\
.171 \\
1.158 \\
10.0\end{array}$ & $\begin{array}{l}0.21056 \\
.154 \\
1.139 \\
6.7\end{array}$ & $\begin{array}{l}0.18339 \\
.139 \\
1.122 \\
4.5\end{array}$ & $\begin{array}{l}0.13399 \\
.110 \\
1.106 \\
2.6\end{array}$ & $\begin{array}{l}0.08706 \\
.091 \\
1.091 \\
1.5\end{array}$ & 0.06211 & 0.20941 & 0.01591 & 0.00835 & 0.00429 & 0.00217 & 0.00109 & 0.00055 \\
\hline 1 & $\begin{array}{l}0.25053 \\
0.402 \\
1.205 \\
4.4\end{array}$ & $\begin{array}{l}.17569 \\
.202 \\
1.185 \\
1.7\end{array}$ & $\begin{array}{l}.03039 \\
.028 \\
1.166 \\
0\end{array}$ & $\begin{array}{l}.00420 \\
.002 \\
1.148 \\
0\end{array}$ & $\begin{array}{l}.05214 \\
.034 \\
1.131 \\
0\end{array}$ & $\begin{array}{l}.09553 \\
.070 \\
1.115 \\
0.2\end{array}$ & $\begin{array}{l}.10665 \\
.093 \\
1.100 \\
0.2\end{array}$ & .09311 & .07008 & .04781 & .03046 & .01849 & .01085 & \\
\hline
\end{tabular}

$q$ Ref. [183] (also includes $q$ for $v^{\prime} \geq 2$ ).

$p$ Ref. [211]; $p$ and $q$ are made equal for $0-0 ; I_{\infty}$ for the strongest band

$r$-centroid ref. [211].

in $v^{\prime}=0$ progression is normalized to 10.0 .

TABLE 62. Franck-Condon factors for the $\mathrm{C}^{1} \Sigma^{+}-\mathrm{A}^{1} \Pi$ Herzberg system

\begin{tabular}{|c|c|c|c|}
\hline$v^{\prime \prime}$ & 0 & 1 & 2 \\
\hline 0 & 0.072 & 0.165 & 0.214 \\
\hline 1 & .211 & .192 & .031 \\
\hline 2 & .293 & .034 & - \\
\hline
\end{tabular}

Data from Leskov (ref. [150]).

TABLE 63. Franck-Condon factors, band strength, r-centroids, and $\mathrm{I}_{\infty}$ for the $\mathrm{b}^{3} \Sigma^{+}-\mathrm{a}^{3} \mathrm{\Pi}$ Third Positive system

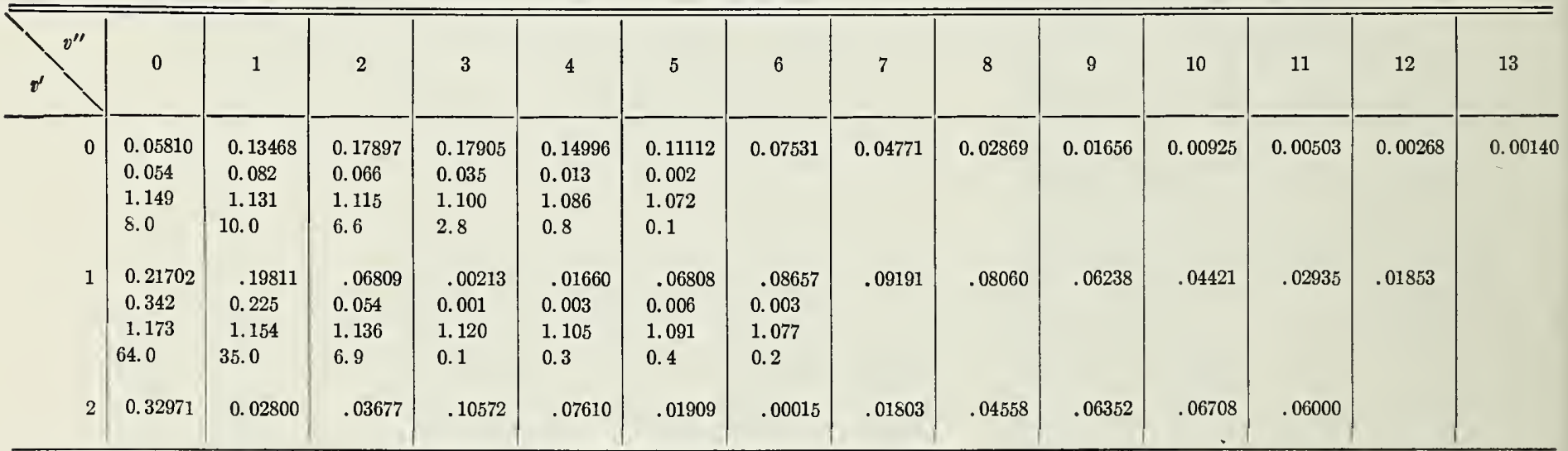

$q$ Ref. [183].

$p$ Ref. [211]. $p$ and $q$ are made $\approx$ equal for the $0-0$ band. $r$-centroid ref. [211].
$\mathrm{I}_{\infty}$ Ref. [211]. $\mathrm{I}_{\infty}$ for the strongest band in the $v^{\prime}=0$ progression is normalized to 10.0 .

TABLE 64. Franck-Condon factors for the $\mathrm{a}^{\prime 3} \Sigma^{+}-\mathrm{a}^{3} \mathrm{II}$ Asundi system

\begin{tabular}{|c|c|c|c|c|c|c|c|}
\hline \multirow{2}{*}{$v^{\prime}$} & \multicolumn{3}{|c|}{$v^{\prime \prime}$} & \multirow{2}{*}{$v^{\prime}$} & \multicolumn{3}{|c|}{$v^{\prime \prime}$} \\
\hline & 0 & 1 & 2 & & 0 & 1 & 2 \\
\hline $0 \ldots$ & $0.03_{7}$ & $0.14_{3}$ & 0.243 & $7 \ldots$ & 0.050 & $0.10_{2}$ & $0.00_{1}$ \\
\hline $1 \ldots$ & $.10_{3}$ & $.19_{5}$ & $.09_{7}$ & $8 \ldots$ & $.03_{6}$ & $.09_{0}$ & $.00_{6}$ \\
\hline 2 & $.16_{2}$ & $.10_{0}$ & $.00_{0}$ & 9 & .021 & $.08_{2}$ & .023 \\
\hline 3 & .181 & .018 & .050 & 10 & $.01_{\theta}$ & .064 & $.04_{1}$ \\
\hline 4 & $.16_{5}$ & $.00_{3}$ & .059 & $11 .$. & $.01_{2}$ & .050 & $.05_{2}$ \\
\hline $5 \ldots$ & $.12 \theta$ & $.03_{\theta}$ & .067 & $12 \ldots$ & $.00_{7}$ & $.03_{8}$ & .055 \\
\hline 6 & $.09_{1}$ & $.08_{1}$ & .024 & & & & \\
\hline
\end{tabular}

Data from Jarmain, Fraser, and Nicholls [114]. 

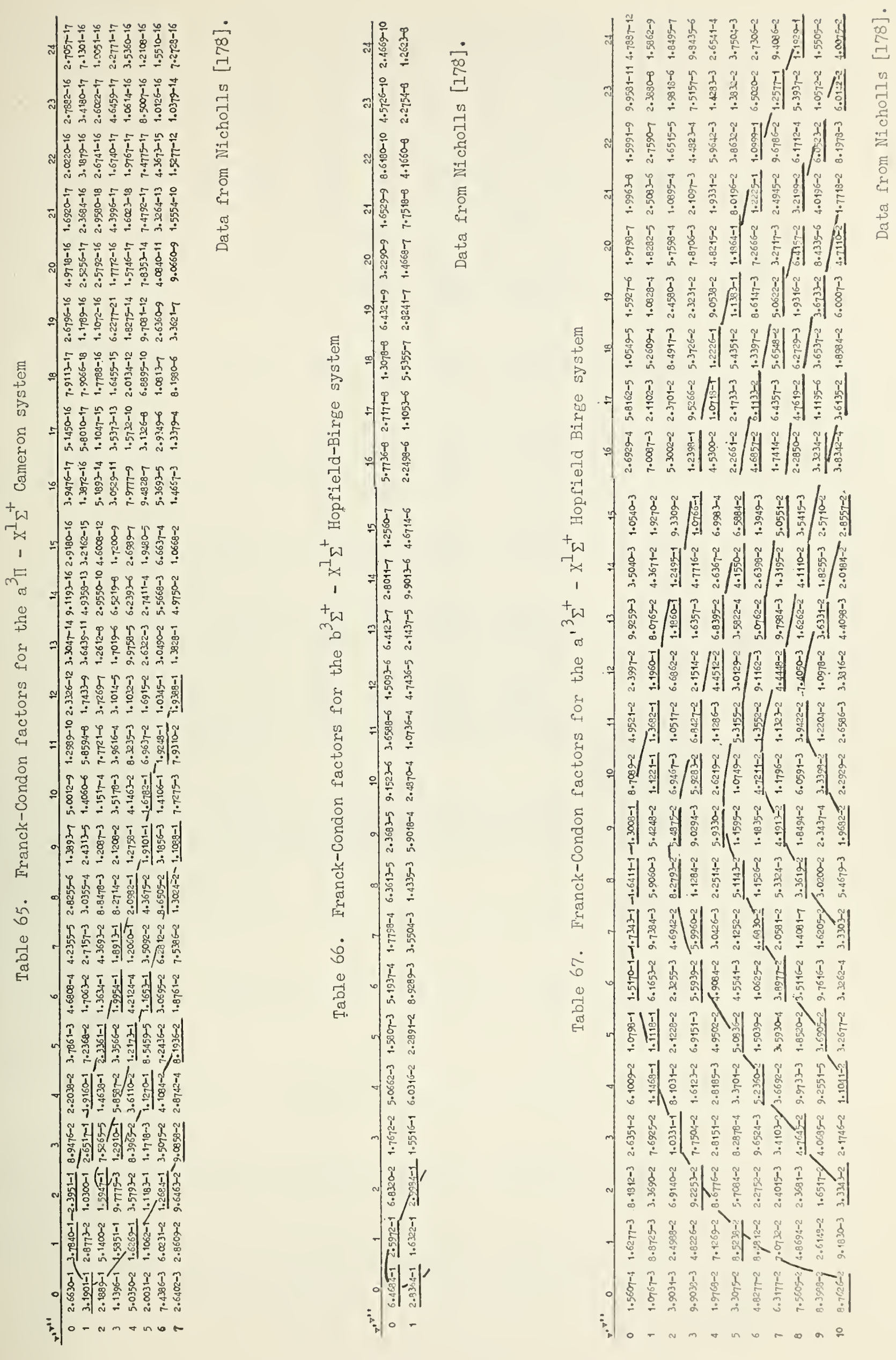

794-90s- (i6- i 
TABLE 68. Franck-Condon factors, $r$-centroids and $\mathrm{I}_{\infty}$ for the $\mathrm{d}^{3} \Delta_{i}-\mathrm{a}^{3}$ II Triplet system

\begin{tabular}{|c|c|c|c|c|}
\hline & 0 & 1 & 2 & 3 \\
\hline \multirow[t]{3}{*}{1} & 0.007 & 0.043 & 0.120 & 0.201 \\
\hline & 1.298 & 1. 321 & 1. 346 & 1. 371 \\
\hline & 1 & 3.4 & 5.1 & 4.0 \\
\hline \multirow[t]{3}{*}{2} & 0.031 & 0.114 & 0.162 & 0.091 \\
\hline & 1. 282 & 1. 305 & 1.328 & 1. 351 \\
\hline & 5.8 & 14.9 & 10.2 & 3.0 \\
\hline \multirow[t]{3}{*}{3} & 0.068 & 0.145 & 0.074 & 0.000 \\
\hline & 1. 267 & 1. 289 & 1. 311 & \\
\hline & 17.0 & 22.8 & 6.9 & \\
\hline \multirow[t]{3}{*}{4} & 0.107 & 0.113 & 0.004 & 0.057 \\
\hline & 1. 252 & 1. 274 & 1.286 & 1. 323 \\
\hline & 35.1 & 23.7 & 0.5 & 4.4 \\
\hline \multirow[t]{3}{*}{5} & 0.133 & 0.051 & 0.018 & 0.079 \\
\hline & 1. 238 & 1. 259 & 1. 287 & 1.305 \\
\hline & 56.3 & 14.3 & 3.3 & 8.7 \\
\hline \multirow[t]{3}{*}{6} & 0.141 & 0.008 & 0.061 & 0.030 \\
\hline & 1.225 & 1. 243 & 1. 269 & 1. 289 \\
\hline & 75.3 & 3.4 & 14.9 & 4.7 \\
\hline \multirow[t]{3}{*}{7} & 0.132 & 0.002 & 0.073 & 0.000 \\
\hline & 1. 212 & 1. 241 & 1. 254 & \\
\hline & 87.7 & 0.8 & 22.7 & {$[-1$} \\
\hline \multirow[t]{3}{*}{8} & 0.113 & 0.022 & 0.045 & 0.020 \\
\hline & 1.200 & 1.220 & 1.252 & 1.267 \\
\hline & 90.2 & 130 & 18.4 & 5.2 \\
\hline \multirow[t]{3}{*}{9} & 0.086 & 0.055 & 0.017 & 0.043 \\
\hline & 1. 186 & 1. 211 & 1. 238 & 1. 240 \\
\hline & 83.2 & 38.4 & 8.4 & 14.6 \\
\hline
\end{tabular}

Q Ref. [240, 239].

$r$-centroids Ref. [240].

$I_{\infty}$ ref. [239].

The quantum number $v^{\prime}$ listed above are one unit higher than that used in refs. $[239,240]$. The tabulated quantities are therefore somewhat uncertain. 


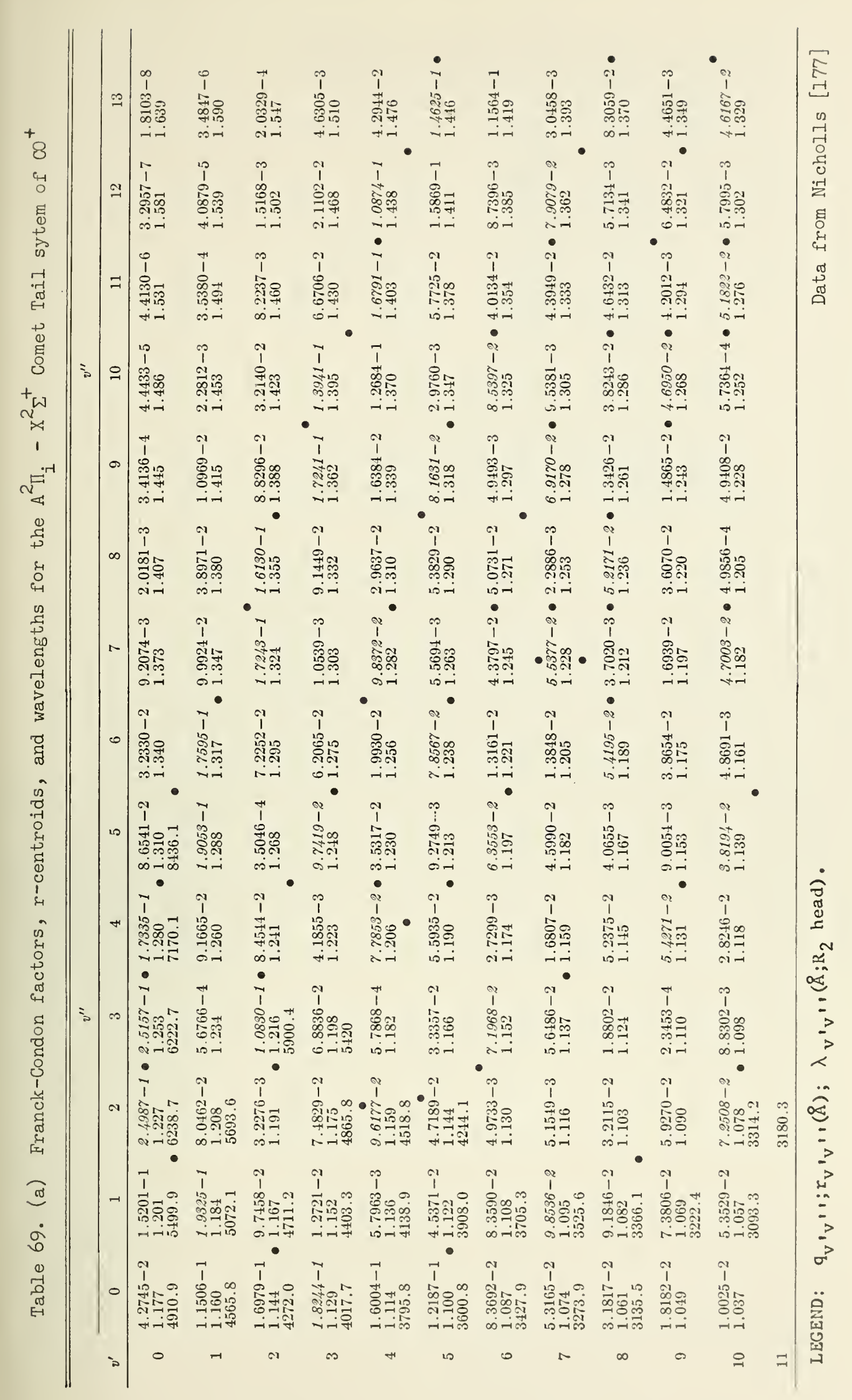


TABLE 69. Continued-(b) Smoothed band strengths and

$\mathrm{I}_{\infty}$ for the $\mathrm{A}^{2} \Pi_{i}-\mathrm{X}^{2} \Sigma^{+}$Comet Tail system of $\mathrm{CO}^{+}$

\begin{tabular}{|c|c|c|c|c|}
\hline$v^{\prime \prime}$ & 0 & 1 & 2 & 3 \\
\hline \multirow[t]{2}{*}{0} & $0.07_{1}$ & $0.21_{\theta}$ & $0.25_{0}$ & \\
\hline & 1.6 & 3.1 & 2.2 & \\
\hline \multirow[t]{2}{*}{1} & $0.18_{8}$ & $0.31_{2}$ & $0.10_{8}$ & \\
\hline & 5.6 & 6.2 & 1.3 & \\
\hline \multirow[t]{2}{*}{2} & 0.255 & $0.16_{2}$ & $0.00_{5}$ & $0.13_{1}$ \\
\hline & 10.0 & 4.3 & - & 1.2 \\
\hline \multirow[t]{2}{*}{3} & $0.23_{8}$ & $0.02_{1}$ & $.12_{4}$ & $0.10_{3}$ \\
\hline & 11.9 & .7 & 2.9 & 1.6 \\
\hline \multirow[t]{2}{*}{4} & 0.169 & $.00_{\theta}$ & $0.16_{8}$ & \\
\hline & 10.6 & .4 & 5.3 & \\
\hline \multirow[t]{3}{*}{5} & $0.09_{5}$ & $.05_{4}$ & 0.073 & \\
\hline & 7.4 & 3.0 & 2.9 & \\
\hline & & & 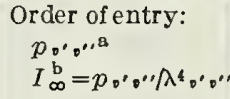 & \\
\hline
\end{tabular}

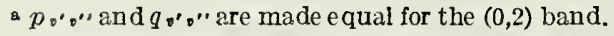

b $I_{\infty}$ for the strongest band in $v^{\prime}=2$ progression is normalized to 10.0 .

Data from Robinson and Nicholls [212]. See also Nicholls [177] who lists absolute band strengths. 


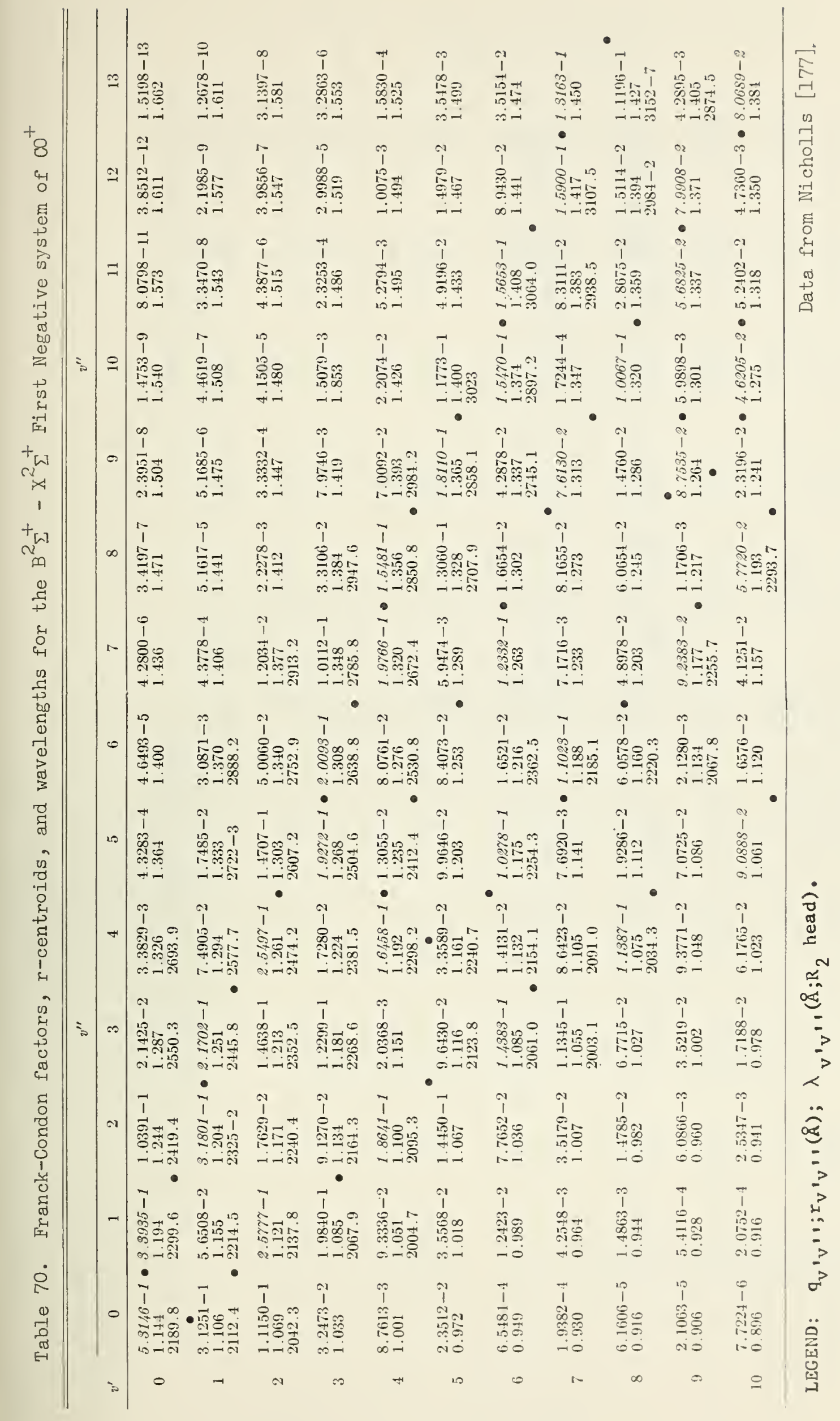




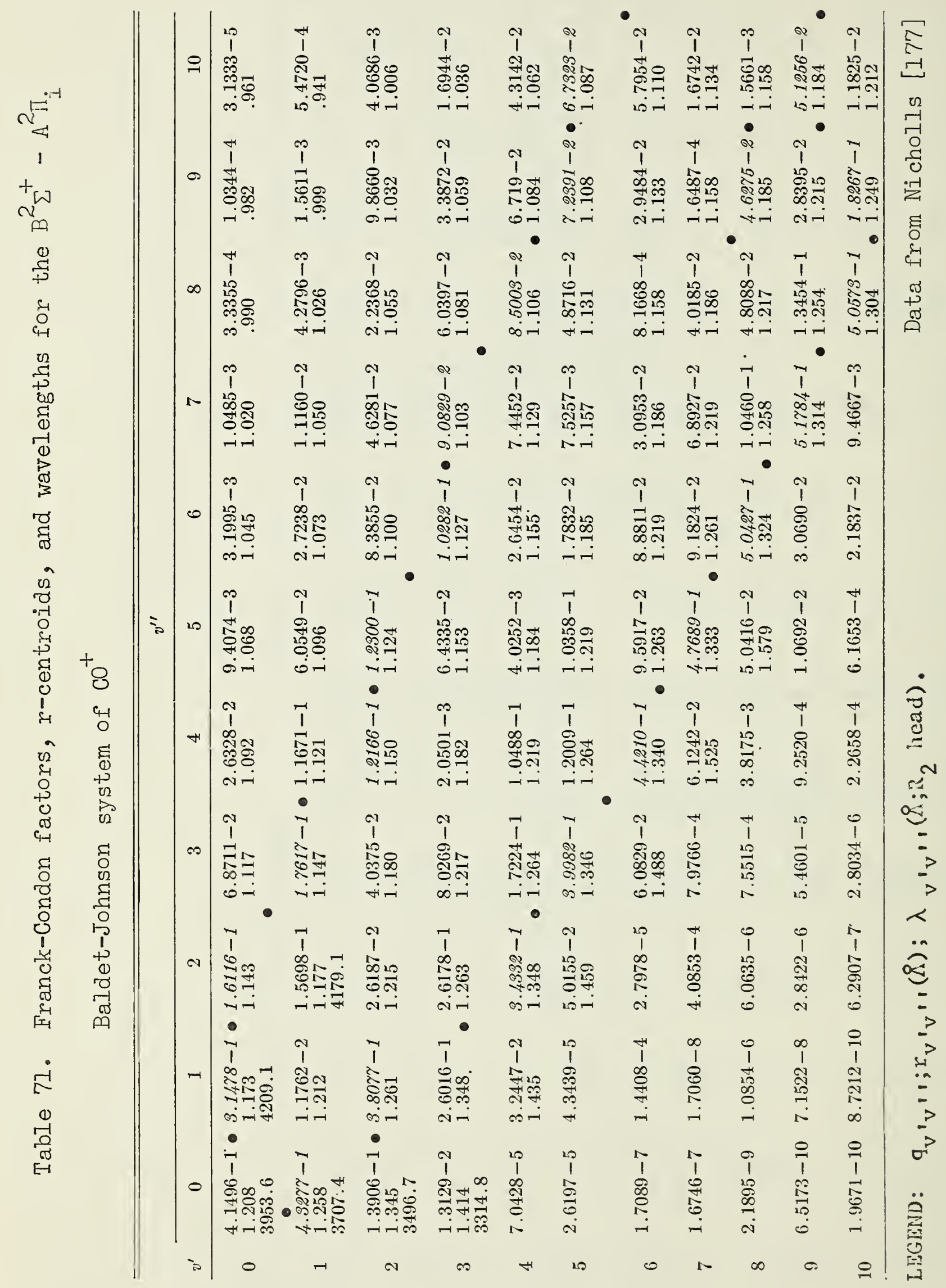


TABLE 72. Franck-Condon factors for ionizing transitions (a) $\mathrm{X}^{2 \Sigma^{+}}\left(\mathrm{CO}^{+}\right) \leftarrow \mathrm{X}^{1} \Sigma^{+}(\mathrm{CO})\left(v^{\prime \prime}=0\right)$

\begin{tabular}{r|r|r}
\hline \multicolumn{1}{r|}{} & \multicolumn{1}{c|}{$q$} & \multicolumn{1}{c}{$\Sigma q$} \\
\hline & & \\
\hline 0 & 0.96355 & 0.96355 \\
1 & .03634 & .99989 \\
2 & .00011 & 1.00000 \\
3 & .00000 & 1.00000 \\
4 & .00000 & 1.00000 \\
5 & .00000 & 1.00000 \\
6 & .00000 & 1.00000 \\
7 & .00000 & 1.00000 \\
8 & .00000 & 1.00000 \\
9 & .00000 & 1.00000 \\
10 & .00000 & 1.00000 \\
\hline
\end{tabular}

(b) $\mathrm{A}^{2} \Pi_{i}\left(\mathrm{CO}^{+}\right) \leftarrow \mathrm{X}^{i} \Sigma^{+}(\mathrm{CO})\left(v^{\prime \prime}=0\right)$

\begin{tabular}{r|r|r}
\hline$v^{\prime}$ & $q$ & $\Sigma q$ \\
\hline & & \\
& & \\
0 & 0.07937 & 0.07937 \\
1 & .17618 & .25555 \\
2 & .21517 & .47072 \\
3 & .19212 & .66284 \\
4 & .14062 & .80346 \\
5 & .08979 & .89320 \\
6 & .05187 & .94507 \\
7 & .02786 & .97293 \\
8 & .01416 & .98709 \\
9 & .00690 & .99399 \\
10 & .00326 & .99725 \\
\hline
\end{tabular}

(c) $\mathrm{B}^{2} \Sigma+\left(\mathrm{CO}^{+}\right) \leftarrow \mathrm{X}^{1} \Sigma^{+}(\mathrm{CO})\left(v^{\prime \prime}=0\right)$

\begin{tabular}{r|r|r}
\hline$v^{\prime}$ & $q$ & $\Sigma q$ \\
\hline & & \\
\hline 0 & 0.68853 & 0.68853 \\
1 & .24825 & .93678 \\
2 & .05248 & .98926 \\
3 & .00895 & .99821 \\
4 & .00146 & .99967 \\
5 & .00026 & .99993 \\
6 & .00005 & .99998 \\
7 & .00001 & .99999 \\
8 & .00000 & .99999 \\
9 & .00000 & .99999 \\
10 & .00000 & .99999 \\
\hline
\end{tabular}

Data from Wacks [264]. Halmann and Laulicht [88a] have calculated $q$ for isotopes $\mathrm{C}^{12} \mathrm{O}^{16}, \mathrm{C}^{13} \mathrm{O}^{16}$, and $\mathrm{C}^{12} \mathrm{O}^{18}$. 
TABLE 73. (a) Lifetimes, Einstein coefficients, and oscillator strengths

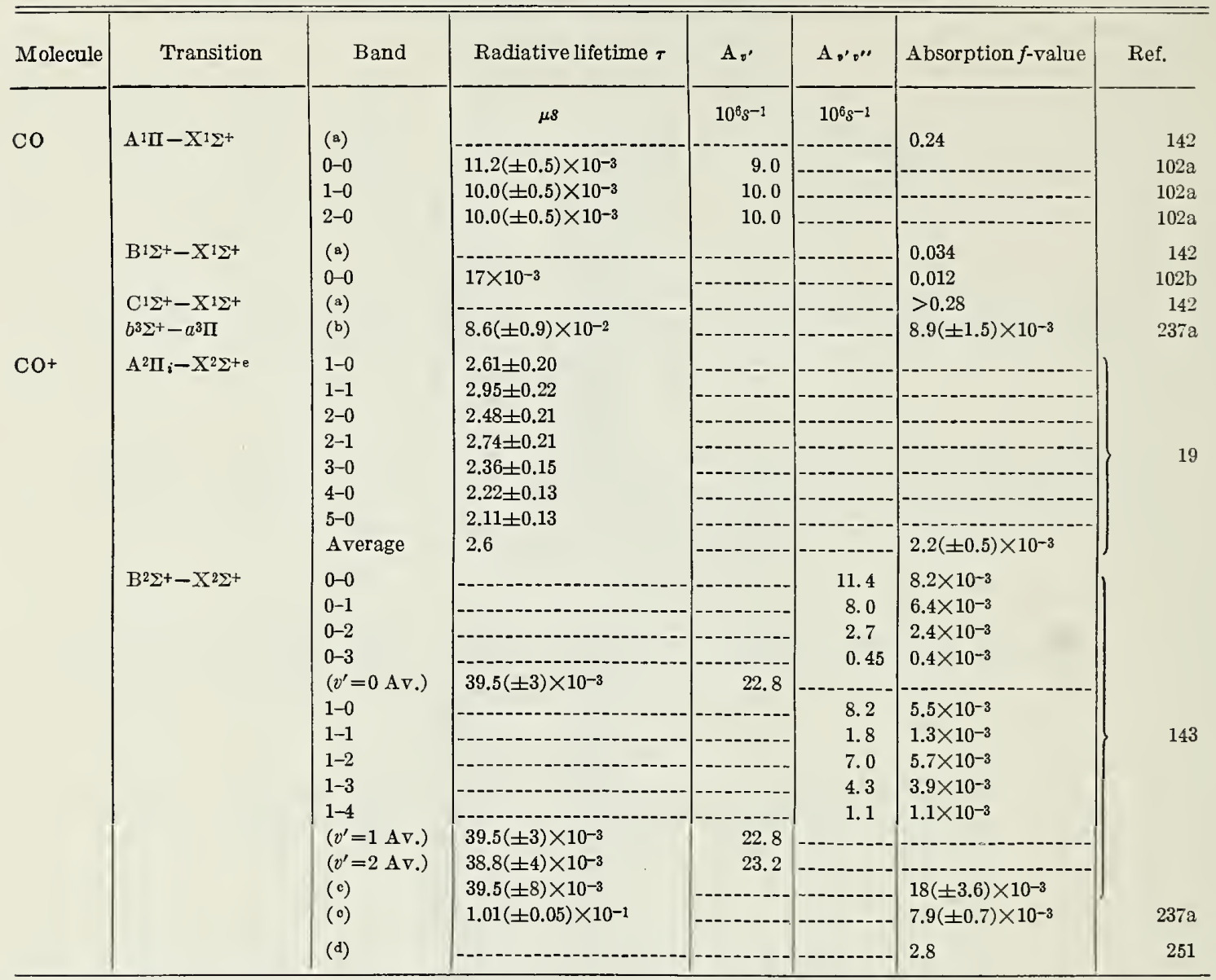

a Integrated $f$-value for the system from inelastic electron scattering. Table 73 (c) lists $f$-values for bands of this system.

b $\tau$ and $f$ for $v^{\prime}=0$

- Average for the B-X transition. The discrepancy between the two values is as yet unexplained.

(b) Einstein coefficients, absolute band strengths, and band oscillator strengths for the $A^{2} \Pi_{i}-X^{2} \Sigma^{+}$Comet Tail system of $\infty^{+}$

\begin{tabular}{|c|c|c|c|c|}
\hline \multirow[b]{2}{*}{$v^{\prime}$} & \multicolumn{4}{|c|}{$v^{\prime \prime}$} \\
\hline & 0 & 1 & 2 & 3 \\
\hline 0 & $\begin{array}{l}4.37+4 \\
1.01-2 \\
1.58-4\end{array}$ & $\begin{array}{l}9.47+4 \\
3.11-2 \\
4.30-4\end{array}$ & $\begin{array}{l}7.63+4 \\
3.66-2 \\
4.45-4\end{array}$ & \\
\hline 1 & $\begin{array}{l}1.42+5 \\
2.65-2 \\
4.44-4\end{array}$ & $\begin{array}{l}1.75+5 \\
4.50-2 \\
6.74-4\end{array}$ & $\begin{array}{l}3.90+4 \\
1.21-2 \\
1.70-4\end{array}$ & \\
\hline 2 & $\begin{array}{l}2.37+4 \\
3.65-2 \\
6.50-4\end{array}$ & $\begin{array}{l}1.13+5 \\
2.33-2 \\
3.75-4\end{array}$ & & $\begin{array}{l}4.33+4 \\
1.76-2 \\
2.26-4\end{array}$ \\
\hline 3 & $\begin{array}{l}2.66+5 \\
3.51-2 \\
6.64-4\end{array}$ & $\begin{array}{l}1.71+4 \\
2.88-3 \\
4.98-5\end{array}$ & $\begin{array}{l}7.84+4 \\
1.78-2 \\
2.78-4\end{array}$ & $\begin{array}{l}4.83+4 . \\
1.52-2 \\
2.13-4\end{array}$ \\
\hline 4 & $\begin{array}{l}2.24+5 \\
2.14-2 \\
4.83-4\end{array}$ & $\begin{array}{l}9.19+3 \\
1.29-2 \\
2.36-4\end{array}$ & $\begin{array}{l}1.33+5 \\
2.42-2 \\
4.07-4\end{array}$ & \\
\hline 5 & $\begin{array}{l}1.48+5 \\
1.36-2 \\
2.88-4\end{array}$ & $\begin{array}{l}6.51+4 \\
7.67-2 \\
1.49-4\end{array}$ & $\begin{array}{l}6.84+4 \\
1.03-2 \\
.1 .85-4\end{array}$ & \\
\hline
\end{tabular}

LEGEIND: $A_{v^{\prime} v^{\prime \prime}}\left(\sec ^{-1}\right) ; p_{v^{\prime} v^{\prime \prime}}\left(a_{o}^{2} e^{2}\right) ; f_{v^{\prime} v^{\prime \prime}}$.

Data from Nicholls [177] d Continuum, 876-374

- Decay of emission from each band observed. Lifetimes of upper vibrational levels measured.

TABLE 73-Continued-(c) Absolute f-values for the $\mathrm{A}^{1} \Pi-\mathrm{X}^{1} \Sigma^{+}$Fourth Positive system

\begin{tabular}{|c|c|c|c|c|c|c|c|c|c|c|c|}
\hline \multicolumn{12}{|c|}{$f_{\nabla / \nabla^{\prime \prime}}\left(\times 10^{-3}\right)$} \\
\hline & 0 & 1 & 2 & 3 & 4 & 5 & 6 & 7 & 8 & 9 & $\Sigma_{0} \cdot f_{0}{ }^{\prime} \cdot \cdots$ \\
\hline 0 & 9.0 & 20.0 & 21.2 & 14.1 & 6.7 & 2.4 & - & --_-. & $\ldots$ & -- & 73.5 \\
\hline 1 & 20.2 & 14.0 & 0.3 & 6.5 & 15.8 & 14.7 & 8.2 & 3.3 & 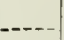 & $\ldots$ & 83.0 \\
\hline 2 & 22.6 & 1.2 & 8.3 & 10.3 & 0.4 & 4.8 & 13.2 & 12.9 & 7.4 & 3.0 & 84.1 \\
\hline
\end{tabular}

Data from Hesser and Dressler [102a]. 
Table 74. Potential energy of the electronic stales of $\mathrm{CO}$ and $\mathrm{CO}^{+*}$

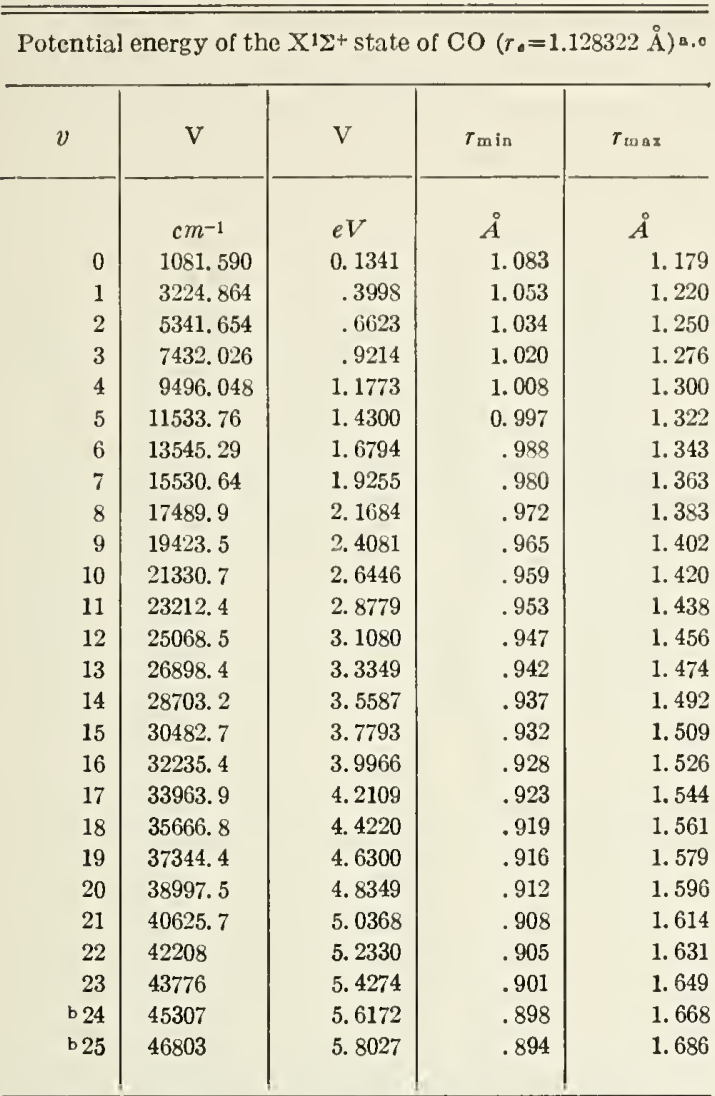

*Data for the $e^{3 \Sigma} \Sigma^{-}$state [138] are not included because of a revision in vibrational quantum numbering [238a]. The newly revised numbering is definitive (sec. 3.10).

B Data for $v=0$ to 7 from vibration-rotation spectra.

b Extrapolated to highest observed level.

- $r_{\bullet}$ : See footnote $\mathrm{X}^{1 \Sigma^{+}}$, table 1.

Data taken from Krupenie and Weissman [138].

Potential energy of the $a^{3} \Pi$ state of CO $\left(\mathrm{T}_{e}=48687.40 \mathrm{~cm}^{-1}=6.0363 \mathrm{eV}, r_{e}=\right.$ $1.2058 \AA$ )

\begin{tabular}{|c|c|c|c|c|c|c|}
\hline$v$ & $\mathrm{~V}$ & V & $\mathrm{T} \cdot+\mathrm{V}$ & $T_{0}+V$ & $r_{\min }$ & $r \max$ \\
\hline & $\mathrm{cm}^{-1}$ & $e V$ & $\mathrm{c}^{m-1}$ & $e V$ & A & 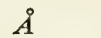 \\
\hline 0 & 868.16 & 0.1076 & 49555.56 & 6.1440 & 1.157 & 1. 263 \\
\hline 1 & 2582.77 & .3202 & 51270.17 & 6. 3565 & 1.124 & 1. 310 \\
\hline 2 & 4266.7 & .5290 & 52954.1 & 6. 5653 & 1. 103 & 1. 344 \\
\hline 3 & 5921.2 & .7341 & 54608.6 & 6. 7704 & 1. 087 & 1. 375 \\
\hline 4 & 7546.8 & .9357 & 56234.2 & 6.9720 & 1. 074 & 1.403 \\
\hline 5 & 9143.3 & 1. 1336 & 57830.7 & 7.1699 & 1. 063 & 1.429 \\
\hline \& 6 & 10710 & 1. 3278 & 59398 & 7. 3642 & 1. 052 & 1.454 \\
\hline a 7 & 12246 & 1. 5183 & 60934 & 7.5547 & 1.043 & 1.478 \\
\hline
\end{tabular}

a Extrapolated to highest observed level.
TABLE 74. Potential energy of the electronic states of $\mathrm{CO}$ and $\mathrm{CO}^{+}-$Continued

Potential energy of the $a^{\prime 3} \mathrm{z}+$ state of $\mathrm{CO}\left(\mathrm{T}_{\mathrm{t}}=55822.92 \mathrm{~cm}^{-1}=6.9210 \mathrm{cV}, r_{\mathrm{c}}=\right.$ $1.3519 \AA)$

\begin{tabular}{|c|c|c|c|c|c|c|}
\hline$v$ & V & v & $T_{0}+V$ & $T_{\bullet}+V$ & $\tau_{\min }$ & $r_{\max }$ \\
\hline & $\mathrm{cm}^{-1}$ & $\mathrm{eV}$ & $c m^{-1}$ & $\mathrm{eV}$ & $\dot{A}$ & i \\
\hline 0 & 612.581 & 0.0759 & 56435.50 & 6. 9969 & 1. 296 & 1. 423 \\
\hline 1 & 1821.44 & .2258 & 57644.36 & 7. 1468 & 1. 257 & 1. 478 \\
\hline 2 & 3008.83 & .3730 & 58831.75 & 7. 2940 & 1. 232 & 1. 520 \\
\hline 3 & 4175.16 & .5176 & 59398.08 & 7.4386 & 1. 214 & 1. 557 \\
\hline 4 & 5321.31 & .6597 & 61144.23 & 7. 5807 & 1. 198 & 1.391 \\
\hline 5 & 6446.32 & .7992 & 62269.24 & 7. 7202 & 1. 185 & 1. 622 \\
\hline 6 & 7552.31 & .9363 & 63375. 23 & 7. 8573 & 1.174 & 1. 653 \\
\hline 7 & 8638. 11 & 1. 0710 & 64461.03 & 7. 9920 & 1.164 & 1.682 \\
\hline 8 & 9705.57 & 1.2033 & 65528.49 & 8. 1243 & 1.155 & 1.711 \\
\hline 9 & 10751.15 & 1. 3329 & 66574.07 & 8. 2539 & 1.147 & 1. 739 \\
\hline 10 & 11779. 26 & 1. 4604 & 67602.18 & 8. 3814 & 1. 139 & 1. 766 \\
\hline 11 & 12788.37 & 1.5855 & 68611.29 & 8. 5065 & 1.132 & 1. 793 \\
\hline 12 & 13778.58 & 1.7083 & 69601.50 & 8. 6293 & 1. 126 & 1.820 \\
\hline 13 & 14750.12 & 1.8287 & 70573.04 & 8. 7497 & 1.120 & 1.847 \\
\hline 14 & 15703.24 & 1.9469 & 71526.16 & 8.8679 & 1.114 & 1.874 \\
\hline 15 & 16637.78 & 2. 0628 & 72460.70 & 8. 9838 & 1.108 & 1.900 \\
\hline 16 & 17554.01 & 2. 1764 & 73376.93 & 9.0974 & 1. 103 & 1. 927 \\
\hline 17 & 18451.41 & 2. 2876 & 74274.33 & 9. 2086 & 1. 099 & 1. 954 \\
\hline 18 & 19330.47 & 2. 3966 & 75153. 39 & 9. 3176 & 1.094 & 1.981 \\
\hline 19 & 20190.91 & 2.5033 & 76013.83 & 9.4243 & 1.090 & 2. 009 \\
\hline 20 & 21032.78 & 2. 6077 & 76855.70 & 9.5287 & 1. 086 & 2.0 \\
\hline 21 & 21855.93 & 2. 7097 & 77678.85 & 9.6307 & 1. 082 & 2.064 \\
\hline 22 & 22660.14 & 2. 8094 & 78483.06 & 9. 7304 & 1. 079 & 2. 092 \\
\hline 23 & 23443. 67 & 2.9066 & 79266.59 & 9. 8276 & 1.075 & 2. 121 \\
\hline
\end{tabular}

\begin{tabular}{|c|c|c|c|c|c|c|}
\hline$v$ & V & $\mathrm{v}$ & $\mathrm{T}_{\bullet}+\mathrm{V}$ & $\mathrm{T}_{0}+\mathrm{V}$ & $r_{\min }$ & $r_{\max }$ \\
\hline & $\mathrm{cm}^{-1}$ & $e V$ & $\mathrm{~cm}^{-1}$ & $e V$ & $\AA$ & A \\
\hline 0 & 574.46 & $0.0 \pi 12$ & 61728.52 & 7. 6532 & 1.309 & 1.440 \\
\hline 1 & 1712 & .2123 & 62866 & 7. 7942 & 1. 270 & $1.49 \mathrm{~S}$ \\
\hline 2 & 2834 & .3513 & 63988 & 7.9333 & 1.245 & 1. 541 \\
\hline 3 & 3940 & .4885 & 65094 & 8.0705 & 1. 226 & 1.579 \\
\hline 4 & 5029 & .6235 & 66183 & 8.2055 & 1. 211 & 1.613 \\
\hline 5 & 6100 & .7563 & 67254 & 8. 3383 & 1.197 & 1. 645 \\
\hline 6 & 7153 & .8869 & 68307 & 8.4688 & 1.186 & $1.6 \% 6$ \\
\hline 7 & 8187 & 1.0151 & 69341 & 8. 5970 & 1.176 & 1.707 \\
\hline 8 & 9202 & 1.1409 & 70356 & 8. 7228 & 1.167 & 1. 730 \\
\hline 9 & 10196 & 1. 2641 & 71350 & 8. 8461 & 1.158 & 1.765 \\
\hline 10 & 11169 & 1. 3848 & 72323 & 8.9667 & 1. 150 & 1. 794 \\
\hline 11 & 12121 & 1.5027 & 73275 & 9.0847 & 1. $1 \$ 3$ & 1. $\$ 23$ \\
\hline 12 & 13050 & 1.6179 & 74204 & 9.1999 & 1.136 & 1.352 \\
\hline 13 & 13956 & 1. 7303 & 75110 & 9.3123 & 1.130 & 1.351 \\
\hline 14 & 14839 & 1.8397 & 75993 & 9.4217 & 1. 124 & 1.910 \\
\hline 15 & 15697 & 1.9461 & 76851 & 9. 5281 & 1. $11 \mathrm{~s}$ & 1.940 \\
\hline 16 & 16530 & 2. 0494 & 77654 & 9.6314 & 1.112 & 1.970 \\
\hline 17 & 17337 & 2. 1495 & 78492 & 9. 7315 & 1. 106 & 2.000 \\
\hline 18 & $1811 \mathrm{~s}$ & 2. 2463 & $792 \pi 3$ & 9. 8243 & 1. 101 & 2. 032 \\
\hline 19 & 18873 & 2. 3395 & 80027 & 9.9218 & 1. 095 & 2.064 \\
\hline 20 & 19599 & 2.4299 & S0753 & $10.011 \mathrm{~s}$ & 1.090 & 2.097 \\
\hline 21 & 20297 & 2.5164 & $\$ 1451$ & $10.09 s 4$ & 1. 0 s4 & 2. 132 \\
\hline 22 & 20966 & 2. 5993 & $\$ 2120$ & 10.1813 & 1. 079 & 2. 167 \\
\hline
\end{tabular}


TABLE 74. Potential energy of the electronic states of $\mathrm{CO}$ and $\mathrm{CO}^{+}$-Continued

Potential energy of the A'II state of $\mathrm{CO}\left(\mathrm{T}_{0}=65074.6 \mathrm{~cm}^{-1}=8.0680 \mathrm{eV}, r_{\mathrm{e}}=\right.$ $1.2352 \AA$ )

\begin{tabular}{|c|c|c|c|c|c|c|}
\hline$v$ & V & V & $T_{0}+V$ & $T_{\bullet}+V$ & $r_{\min }$ & $f_{\max }$ \\
\hline & $\mathrm{cm}^{-1}$ & $e V$ & $\mathrm{~cm}^{-1}$ & $e V$ & $\AA$ & $\AA$ \\
\hline 0 & 753.49 & 0.0934 & 65828.1 & 8. 1614 & 1. 183 & 1. 297 \\
\hline 1 & 2242.3 & .2780 & 67316.9 & 8. 3460 & 1. 148 & 1. 348 \\
\hline 2 & 3685.2 & . 4569 & 68759.8 & 8. 5249 & 1.126 & 1. 387 \\
\hline 3 & 5097.9 & . 6320 & 70172.5 & 8. 7001 & 1. 110 & 1.422 \\
\hline 4 & 6476.1 & .8029 & 71550.7 & 8.8709 & 1.096 & 1. 454 \\
\hline 5 & 7818.2 & . 9693 & 72892.8 & 9.0373 & 1.085 & 1. 484 \\
\hline 6 & 9125.0 & 1.1313 & 74199.6 & 9. 1993 & 1. 075 & 1. 514 \\
\hline 7 & 10401.8 & 1. 2896 & 75476.4 & 9.3576 & 1. 066 & 1. 542 \\
\hline 8 & 11641.0 & 1.4433 & 76715.6 & 9.5113 & 1. 058 & 1.571 \\
\hline 9 & 12846.9 & 1.5928 & 77921.5 & 9. 6608 & 1.051 & 1. 599 \\
\hline 10 & 14018.8 & 1.7381 & 79093.4 & 9. 8061 & 1.044 & 1. 627 \\
\hline 11 & 15155.6 & 1.8790 & 80230.2 & 9.9470 & 1.038 & 1. 656 \\
\hline 12 & 16262 & 2.0162 & 81336 & 10.0841 & 1. 032 & 1. 685 \\
\hline 13 & 17333 & 2.1490 & 82407 & 10.2169 & 1. 027 & 1.714 \\
\hline 14 & 18371 & 2.2777 & 83445 & 10.3456 & 1.022 & 1.743 \\
\hline 15 & 19366 & 2.4010 & 84440 & 10.4690 & 1.017 & 1.773 \\
\hline 16 & 20330 & 2.5205 & 85404 & 10.5885 & 1.013 & 1.804 \\
\hline 17 & 21257 & 2. 6355 & 86331 & 10.7034 & 1.009 & 1.837 \\
\hline 18 & 22138 & 2.7447 & 87212 & 10.8126 & 1.005 & 1.871 \\
\hline в 19 & 22970 & 2.8478 & 88045 & 10.9159 & 1.001 & 1.909 \\
\hline a 20 & 23762 & 2.9460 & 88837 & 11.0141 & 0.996 & 1. 951 \\
\hline
\end{tabular}

axtrapolated to highest observed level.

Potential energy of the I ${ }^{1} \Sigma-$ state of $\mathrm{CO}\left(\mathrm{T}_{\mathrm{e}}=66185 \mathrm{~cm}^{-1}=8.2057 \mathrm{eV}, r_{\mathrm{e}}=\right.$ $1.416 \AA$ )

\begin{tabular}{|c|c|c|c|c|c|c|}
\hline$v$ & $\mathrm{~V}$ & $\mathrm{~V}$ & $T_{0}+\mathrm{V}$ & $\mathrm{T}_{0}+\mathrm{V}$ & $r_{\min }$ & $r_{\max }$ \\
\hline & $c m^{-1}$ & $e V$ & $\mathrm{~cm}^{-1}$ & $e V$ & $\stackrel{\circ}{A}$ & A \\
\hline 0 & 530 & 0.066 & 66715 & 8. 271 & 1.35 & 1.48 \\
\hline 1 & 1580 & 196 & 67760 & 8. 401 & 1.29 & 1.53 \\
\hline 2 & 2600 & .324 & 68790 & 8. 528 & 1.25 & 1. 56 \\
\hline 3 & 3610 & .448 & 69790 & 8.653 & 1. 22 & 1.59 \\
\hline 4 & 4600 & .571 & 70780 & 8.776 & 1.19 & 1.61 \\
\hline 5 & $\mathbf{5 5 6 0}$ & .690 & 71750 & 8.896 & 1. 16 & 1.64 \\
\hline 6 & 6510 & .808 & 72700 & 9.013 & 1. 14 & 1.66 \\
\hline 7 & 7450 & .924 & 73630 & 9. 129 & 1. 12 & 1.68 \\
\hline 8 & 8360 & 1. 037 & 74540 & 9.242 & 1.09 & 1.69 \\
\hline 9 & 9250 & 1. 148 & 75440 & 9.353 & 1.07 & 1.71 \\
\hline 10 & 10120 & 1.257 & 76310 & 9.461 & 1.05 & 1. 73 \\
\hline 11 & 10980 & 1.363 & 77160 & 9. 567 & 1.03 & 1.75 \\
\hline 12 & 11810 & 1.467 & 78000 & 9.670 & 1.01 & 1. 77 \\
\hline
\end{tabular}

Potential energy of the $\mathrm{B}^{1} \Sigma^{+}$state of $\mathrm{CO}\left(\mathrm{T}_{\mathrm{o}}=86928 \mathrm{~cm}^{-1}=10.7774 \mathrm{eV}\right.$, $r_{0}=1.120 \AA$ ) $\mathrm{a}$

\begin{tabular}{r|r|r|r|r|r|r}
\hline$v$ & $\mathrm{~V}$ & $\mathrm{~V}$ & $\mathrm{~T}+\mathrm{V}$ & $\mathrm{T} \bullet+\mathrm{V}$ & $r_{\text {m in }}$ & \multicolumn{1}{c}{$r_{\text {max }}$} \\
\hline & & & & & & \\
\hline & $c m^{-1}$ & $e V$ & $c m^{-1}$ & $e V$ & $\AA$ & $\AA$ \\
0 & 1072 & 0.1329 & 87998 & 10.9101 & 1.08 & 1.17 \\
1 & 3154 & .3910 & 90080 & 11.1682 & 1.05 & 1.22 \\
2 & 5154 & .6390 & 92080 & 11.4162 & 1.03 & 1.25 \\
\hline
\end{tabular}

a Curve taken from ref. [258] but with different Z.P.E.
TABLE 74. Potential energy of the electronic states of $\mathrm{CO}$ and $\mathrm{CO}^{+}-$Continued

Potential energy of the $\mathrm{X}^{2} \Sigma^{+}$state of $\mathrm{CO}^{+}\left(\mathrm{T}_{\bullet}=113007 \mathrm{~cm}^{-1}=14.0108 \mathrm{eV}\right.$, $r_{0}=1.11516 \AA$ )

\begin{tabular}{|c|c|c|c|c|c|c|}
\hline$v$ & $\mathrm{~V}$ & V & $T_{0}+V$ & $T_{\bullet}+V$ & $r_{\min }$ & $r_{\max }$ \\
\hline & $\mathrm{cm}^{-1}$ & eV & $\mathrm{cm}^{-1}$ & $\mathrm{eV}$ & $\AA$ & $\AA$ \\
\hline 0 & 1103.33 & 0.1368 & 114110.33 & 14.1475 & 1.071 & 1.165 \\
\hline 1 & 3287.22 & 0.4076 & 116294.22 & 14.4183 & 1.041 & 1.206 \\
\hline 2 & 5440.80 & 0.6746 & 118447.80 & 14. 6853 & 1.023 & 1.236 \\
\hline 3 & 7564.01 & 0.9378 & 120571.01 & 14.9486 & 1.008 & 1.263 \\
\hline 4 & 9656.94 & 1. 1973 & 122663.94 & 15.2080 & 0.996 & 1.286 \\
\hline 5 & 11719.47 & 1.4530 & 124726.47 & 15. 4637 & 0.986 & 1.309 \\
\hline 6 & 13751.63 & 1.7049 & 126758.63 & 15.7157 & 0.977 & 1.330 \\
\hline 7 & 15753.47 & 1.9531 & 128760.47 & 15. 9639 & 0.968 & 1.350 \\
\hline 8 & 17724.95 & 2. 1976 & 130731.95 & 16.2083 & 0.961 & 1.370 \\
\hline 9 & 19666.6 & 2.4383 & 132673.6 & 16. 4490 & 0.954 & 1.390 \\
\hline 10 & 21578.2 & 2.6753 & 134585.2 & 16.6860 & 0.948 & 1.409 \\
\hline 11 & 23459.2 & 2.9085 & 136466.2 & 16.9193 & 0.943 & 1.428 \\
\hline 12 & 25310.0 & 3. 1380 & 138317.0 & 17.1487 & 0.938 & 1.447 \\
\hline 13 & 27102.3 & 3. 3602 & 140109.3 & 17. 3709 & 0.933 & 1.466 \\
\hline
\end{tabular}

Potential energy of the $\mathrm{A}^{2} \Pi_{i}$ state of $\mathrm{CO}^{+}\left(\mathrm{T}_{e}=133740 \mathrm{~cm}^{-1}=16.5813 \mathrm{eV}\right.$, $r_{0}=1.24378 \AA$ )

\begin{tabular}{|c|c|c|c|c|c|c|}
\hline$v$ & V & V & $\mathrm{T} \cdot+\mathrm{V}$ & $T_{0}+\mathrm{V}$ & $r_{\min }$ & $r_{\max }$ \\
\hline & $\mathrm{cm}^{-1}$ & $e V$ & $\mathrm{~cm}^{-1}$ & $\mathrm{eV}$ & $\AA$ & $\dot{A}$ \\
\hline 0 & 777.65 & 0.0964 & 134517.65 & 16.6777 & 1. 191 & 1.3 \\
\hline 1 & 2312.88 & .2868 & 136052.88 & 16. 8680 & 1. 157 & 1. 354 \\
\hline 2 & 3820.74 & .4737 & 137560.74 & 17.0550 & 1.136 & 1. 391 \\
\hline 3 & 5301.83 & .6573 & 139041.83 & 17. 2386 & 1.119 & 1.42 \\
\hline 4 & 6756.38 & .8377 & 140496.38 & 17.4189 & 1. 105 & 1.4 \\
\hline 5 & 8184.11 & 1. 0147 & 141924.11 & 17. 5959 & 1. 094 & 1. 481 \\
\hline 6 & 9585.20 & 1. 1884 & 143325.20 & 17. 7697 & 1. 083 & 1. 508 \\
\hline 7 & 10959.79 & 1. 3588 & 144699.79 & 17. 9401 & 1. 074 & 1.534 \\
\hline 8 & 12307.90 & 1.5259 & 146047.90 & 18. 1072 & 1. 066 & 1.5 \\
\hline 9 & 13629.46 & 1.6898 & 147369.46 & 18. 2711 & 1.059 & 1. 585 \\
\hline 10 & 14924. 72 & 1.8504 & 148664.72 & 18.4317 & 1. 052 & 1.610 \\
\hline 11 & 16193.82 & 2. 0077 & 149933.82 & 18. 5890 & 1. 046 & 1. 634 \\
\hline
\end{tabular}

Potential energy of the $\mathrm{B}^{2} \Sigma^{+}$state of $\mathrm{CO}^{+}\left(\mathrm{T}_{\mathrm{a}}=158884 \mathrm{~cm}^{-1}=19.6987 \mathrm{eV}\right.$, $r_{0}=1.16878 \AA$ )

\begin{tabular}{|c|c|c|c|c|c|c|}
\hline 0 & $\mathrm{~V}$ & V & $\mathrm{T}_{0}+\mathrm{V}$ & $T_{e}+V$ & $\tau_{\min }$ & $r_{\max }$ \\
\hline & $c m^{-1}$ & $e V$ & $\mathrm{~cm}^{-1}$ & $e V$ & $\AA$ & $\dot{A}$ \\
\hline 0 & 860.15 & 0.1066 & 159744.15 & 19.8053 & 1. 120 & 1. 227 \\
\hline 1 & 2539. 55 & .3149 & 161423.55 & 20.0135 & 1.089 & 1. 277 \\
\hline 2 & 4166. 05 & .5165 & 163050.05 & 20. 2152 & 1. 069 & 1.315 \\
\hline 3 & 5741.62 & .7119 & 164625.62 & 20.4105 & 1.054 & 1. 349 \\
\hline 4 & 7268.23 & 9011 & 166152.23 & 20. 5998 & 1.042 & 1. 381 \\
\hline 5 & 8749.8 & 1.0848 & 167633.8 & 20.7835 & 1.032 & 1. 411 \\
\hline 6 & 10186.9 & 1.2630 & 169070.9 & 20.9616 & 1.023 & 1.441 \\
\hline 7 & 11588.7 & 1.4368 & 170472.7 & 21. 1354 & 1. 015 & 1. 471 \\
\hline 8 & 12920.7 & 1.6019 & 171804.7 & 21.3006 & 1.008 & 1.500 \\
\hline 9 & 14193.8 & 1.7598 & 173077.8 & 21.4584 & 1.001 & 1.531 \\
\hline a 10 & 15390.1 & 1. 9081 & 174274.1 & 21.6067 & 0.993 & 1. 564 \\
\hline
\end{tabular}

- Extrapolated to highest observed level. 
Figure 1. Potential energy curves for $\mathrm{CO}$ and $\mathrm{CO}+$.
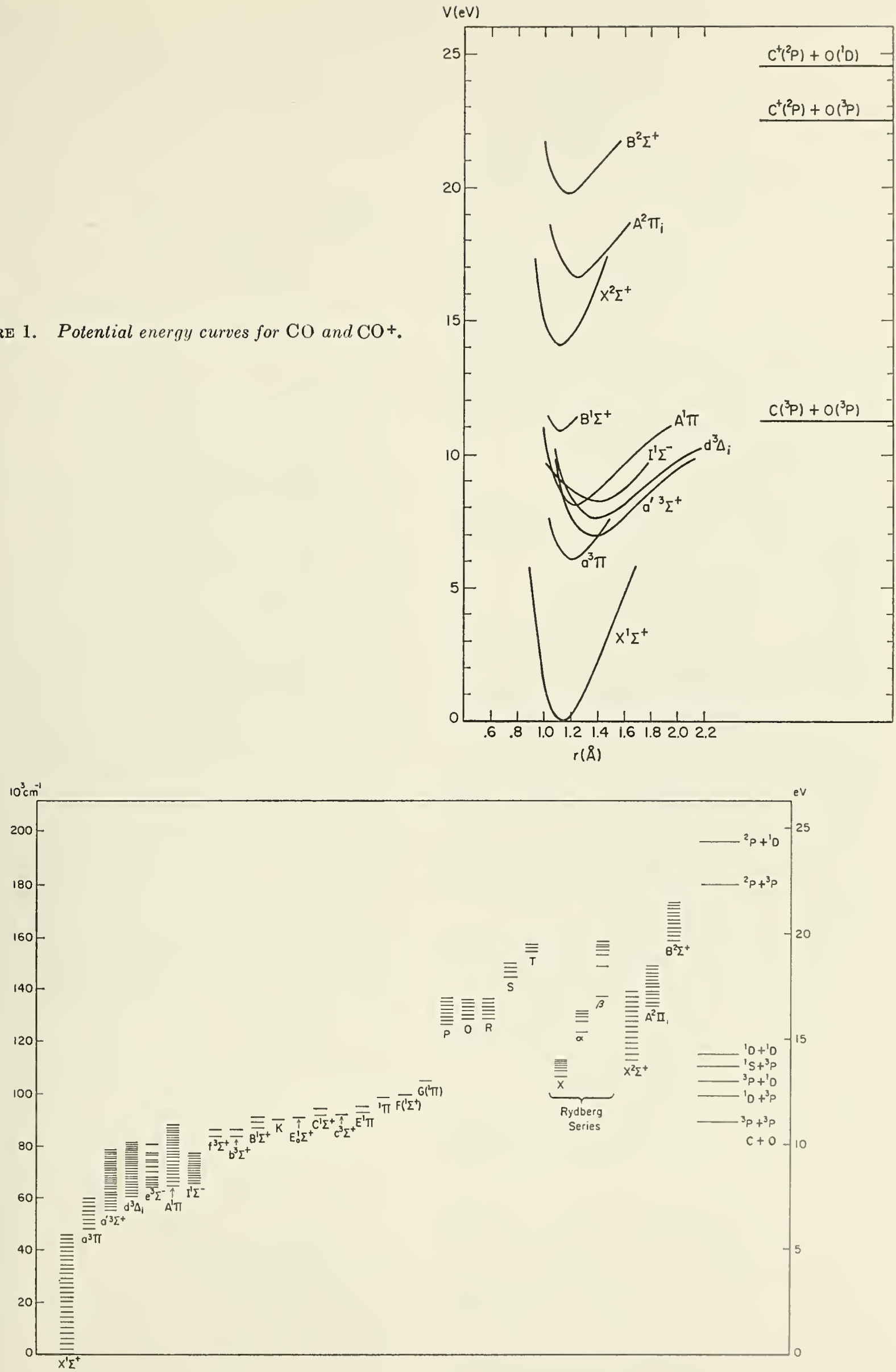

Frgure 2. Energy level diagram for $\mathrm{CO}$ and $\mathrm{CO}^{+}$. 


\section{References}

The references cited below have been traced from a number of standard sources [99, 187, 213, 117,69]. Recent references have been traced through the use of:

(a) Herzberg, G., and Howe, L. L., "Bibliography of Spectra of Diatomic Molecules, 1950-1960", Ottawa.

(b) Mulliken, R. S., "Bibliography on Diatomic Molecules, 1950-1960", Chicago.

(c) Phillips, J. G., and Davis, S. P., Newsletter, "Analysis of Molecular Spectra", Berkeley.

These sources have been supplemented by the use of Current Contents and Chemical Abstracts (Vol. 1 to the present). The literature search terminated in May, 1965. Most valuable as general references are the books by Herzberg [99], Johnson [120], and Jevons [117], the latter having recently been republished by University Microfilms. A review by Birge [23] in 1926 is the most convenient source for tracing the earliest works on CO spectra, many of which by now are mainly of historical interest.

[1] Amaldi, E., On the Raman effect of CO, Z. Physik 79, 492-94 (1932).

[2] Anand, B. M., Bands forming Rydberg series and ionization potential of carbon monoxide, Sci. Cult. (Calcutta) 8, 278-79 (1942).

[3] Asundi, R. K., A new band system of carbon monoxide, Nature 123, 47-8L (1929).

[4] Asundi, R. K., The third positive carbon and associated bands, Proc. Roy. Soc. (London), Ser. A: 124, 277-96 (1929).

[5] Asundi, R. K., Rotational analysis of the Angstrom bands at $\lambda \lambda 6080$ and $6620 \AA$.U., Proc. Indian Acad. Sci., Sect. A. 3, 554-61 (1936).

[6] Asundi, R. K., A new comet tail band, Current Sci. (India) 9, 503-4L (1940).

[7] Asundi, R. K., New bands in the triplet carbon system, Proc. Indian Acad. Sci., Sect. A. 3, 491-93 (1940).

[8] Baldet, F., The spectra of the thermionic discharge in carbon monoxide, Compt. Rend. 178, 1525-27 (1924).

[9] (a) Baldet, F., The spectra of carbon monoxide at very low pressure, and the spectra of comet tails, Compt. Rend. 180, 271-73 (1925).

(b) Baldet, F., On the third negative group of carbon and the spectra of comet tails. Extension to the red and structure of the bands, Compt. Rend. 180, 820-22 (1925).

[10] Baldet, F., The band spectra associated with carbon, Nature 116, 360 (1925).

[11] Barbier, D., Presence in the night sky spectrum of unidentified new bands, Intern. Conf. (July, 1947) Gassiot Comm., Roy. Soc. London, 8-12 (1948). (C.A. 44, 5699c).

[12] Barrow, R. F., Triplet bands of carbon monoxide: the system $e^{3} \Sigma^{-}-a^{3} \Pi$, Nature 189, 480-1L (1961).

[13] Barrow, R. F., Gratzer, W. B., and Malherbe, J. F., Intensities of bands in the system $b^{3} \Sigma^{+}-a^{3} \Pi$ of carbon monoxide, Proc. Phys. Soc. (London) A69, 574-6 (1956).

[14] Bates, D. R., Relative transition probabilities in band systems of diatomic molecules, Monthly Notices Roy. Astron. Soc. 112, 614-36 (1952).
[15] Bedard, F. D., Gallagher, J. J., and Johnson, C. M., Microwave measurement of $\mathrm{D}_{0}$ for $\mathrm{CO}$, Phys. Rev. 92, 1440 (1953).

[16] Beer, B. S., Rotational analysis of the $0 \rightarrow 4,0 \rightarrow 5$, $1 \rightarrow 4$, and $1 \rightarrow 5$ bands of the III. positive system of CO, Z. Physik 10\%, 73-85 (1937).

[17] Benedict, W. S. (private communication).

[18] Benedict, W. S., Herman, R., Moore, G. E., and Silverman, S., The strengths, widths, and shapes of lines in the vibration-rotation bands of $\mathrm{CO}$, Astrophys. J. 135, 277-37 (1962).

[19] Bennett, R. G., and Dalby, E. W., Experimental oscillator strength of the comet-tail system of $\mathrm{CO}^{+}$, J. Chem. Phys. 32, 1111-13 (1960).

[20] Bhagavantam, S., Raman effect in gases: $\mathrm{CO}$ and NO, Phys. Rev. 42, 437L (1932).

[21] Birge, R.T., The band spectra associated with carbon, Nature 116, 170-1L (1925).

[22] Birge, R. T., The energy levels of the carbon monoxide molecule, Nature 11\%, 229-30 (1926).

[23] Birge, R. T., The band spectra of carbon monoxide, Phys. Rev. 28, 1157-81 (1926).

[24] Biskamp, H., Study of the first negative group of $\mathrm{CO}^{+}$, Z. Physik 86, 33-41 (1933).

[25] Blackburn, C. M., Analysis of the "comet tail" bands, (abstract) Phys. Rev. 25, 888 (1925).

[26] Blackburn, C. M., An application of the quantum theory of band spectra to the first negative Deslandres group of carbon, Proc. Nat. Acad. Sci. U.S. 11, 28-34 (1925).

[27] Brewer, L., Gilles, P. IV., and Jenkins, F. A., The vapor pressure and heat of sublimation of graphite, J. Chem. Phys. 16, 797-807 (1948).

[28] Brewer, L., and Searcy, A. IV., High Temperature Chemistry, Ann. Revs. Phys. Chem. y, 259-86 (1956).

[29] Brion, H., and Moser, C., Electronic structure of carbon monoxide, J. Chem. Phys. 32, 1194-9 (1960).

[30] Brons, F., Predissociation in the third positive group of CO, Nature 135, 873 (1935).

[31] Brons, F., Predissociation.in the $A^{1} \Pi$ level of $\mathrm{CO}$ and the dissociation energy of this molecule, Physica 2, 1108-13 (1935).

[32] (a) Budó, A., On the triplet-band term formulas for the general intermediate case and application to the $\mathrm{B}^{3} \Pi-, \mathrm{C}^{3} \mathrm{II}$ - terms of the $\mathrm{N}_{2}$ molecule, $\mathrm{Z}$. Physik 96, 219-29 (1935).

(b) Budo, A., The rotational constants B, D, and $\mathrm{Y}$ of the ${ }^{3} \Pi$-terms of $\mathrm{TiO}, \mathrm{C}_{2}, \mathrm{CO}, \mathrm{PH}, \mathrm{AlH}$, NH, Z. Physik 98, 437-44 (1936).

[33] Budó A., and Kovács, I., On the singlet-triplet perturbations in band spectra, Z. Physik 109, 393-402 (1938).

[34] (a) Bulthuis, H., Dissertation (Groningen) (1935).

(b) Bulthuis, H., The band spectrum of $\mathrm{CO}^{+}$, Physica 1, 873-80 (1934).

[35] Bulthuis H., The spectrum of $\mathrm{CO}^{+}$, Proc. Acad. Sci. Amsterdam 38, 604-17 (1935).

[36] Burrus, C. A., Stark effect from 1.1 to 2.6 millimeters 
wavelength: $\mathrm{PII}_{3}, \mathrm{PD}_{3}, \mathrm{DI}$, and $\mathrm{CO}, \mathrm{J}$. Chem. Phys. 28, 427-29 (1958).

[37] Burrus, C. A., Zeeman effect in the 1- to 3-millimeter wave region: inolecular $g$ factors of several light molecules, J. Chem Phys. 30, 976-83 (1959).

[38] (a) Cabannes, J., and Rousset, A., Raman effect in gases under normal pressure, Compt. Rend. 206, 85-8 (1937).

(b) Cabannes, J., and Rousset, A., The Raman effect in gases at atmospheric pressure. III., J. Phys. Rad. [8] 1, 210-16 (1940).

[39] Cameron, W. H. B., The production of some spectra of carbon, oxygen, and nitrogen in the presence of neon, Phil. Mag. 1, 405-17 (1926).

[40] Carroll, P. K., Structure of the triplet bands of CO, J. Chem. Phys. 36, 2861-9 (1962).

[41] Childs, D. R., Vibrational wave functions and overlap integrals of various band sytems, Research Report 147, AVCO-Everett Research Lab., AVCO Corp., Everett, Mass. (Jan., 1963) 24 pp.

[42] Codling, K. (private communication).

[43] Coster, D., and Brons, F., Predissociation in the Ångstrom bands of CO, Physica 1, 155-60 (1934).

[44] Coster, D., and Brons, F., On the Angstrom bands of CO, Physica 1, 634-48 (1934).

[45] Coster, D., Brons, II. H., and Bulthuis, H., The band spectrum of $\mathrm{CO}^{+}$, Z. Physik 79, 787-822 (1932).

[46] Coulson, C. A., Valence, Second Edition, (Oxford University Press, London, 1961).

[47] Cowan, M. J., Microwave spectroscopy in the 0.5 to 1.5 millimeter wave region: $\mathrm{Hl}, \mathrm{DCl}, \mathrm{DBr}, \mathrm{DI}$, and CO (Ph.D. Thesis), Dept of Physics, Duke University (1959).

[48] Cowan, M., and Gordy, W., Further extension of microwave spectroscopy in the submillimeter wave region, Phys. Rev. 104, 551-2L (1956).

[49] Cox, J. T., and Gordy, W., Zeeman effect of some linear and symmetric-top molecules, Phys. Liev. 101, 1298-1300 (1956).

[50] Crawford, F. H., The Zeeman effect in the Angstrom CO bands II., Phys. Rev. 33, 341-53 (1929).

[51] Crawford, F. H., Zeeman effect in diatomic molecuiar spectra, Rev. Mod. Phys. 6, 90-117 (1934).

[52] Deutsch, J. L., and Barrow, R. F., The state $I^{1} \Sigma^{-}$of carbon monoxide, Proc. Phys. Soc. (London) 80, 561-3 (1962).

[53] Dieke, G. H., Internal resonance in the $\mathrm{CO}$ molecule (abstract), Phys. Rev. 43, 780 (1933).

[54] (a) Dieke, G. H., and Mauchly, J. W., Structure of the third positive group of $\mathrm{CO}$ bands, Nature 129, 546 (1932).

(b) Dieke, G. H., and Mauchly, J. W., The structure of the third positive group of CO bands, Pliys. Rev. 43, 12-30 (1933).

[55] Dorman, F. H., and Morrison, J. D., Double and triple ionization in molecules induced by electron impact, J. Chem. Phys. 35, 575-81 (1961).

[56] Douglas, A. E., and Møller, C. K., Predissociations of the $\mathrm{C}^{12} \mathrm{O}$ and $\mathrm{C}^{13} \mathrm{O}$ molccules, Can. J. Phys. 33, 125-32 (1955).

[57] (a) Duffendack, O. S., and Fox, G. W., Energy levels of the cartion monoxide molecule, Nature 118, 12-13 (1926).

(b) Duffendack, O. S., and Fox, G. W., Radiating poientials of the band systems of cartuon monoxide, Science 61, 277-8 (1926).

[58] Duffendack, O. S., and Fox, G. W., The excitation of the spectra of carbon monoxide by electron impacts, Astrophys. J. 65, 214-37 (1927).

[59] Duncan, D. C., The excitation of the spectra of nitrogen by electron impacts, Astrophys. J. 62, 145-67 (1925).

[60] Estey, R. S., New measurements in the fourth positive CO bands, Phys. Rev. 35, 309-14 (1930).

[61] Evans, W., and Wagman, D. D. (private communication).

[62] Fineman, M. A., and Petrocelli, A. W., Electron impact study of $\mathrm{CO}$ using a Lozier apparatus, J. Chem. Phys. 36, 25-32 (1962).

[63] (a) Fishburne, E. S., Weinberg, MI., Edse, IR., and Rao, K. N., Spectral emission from a $\mathrm{C}_{2} \mathrm{~N}_{2}+$ $\mathrm{N}_{2} \mathrm{O}$ flame, Paper 05, Symposium on Molecular Structure and Spectra, Ohio State University, Columbus, Ohio (June 15-19, 1964).

(b) Weinberg, J. M., Fishburne, E. S., and Rao, K. N., "Hot" bands of $\mathrm{CO}$ at $4.7 \mu$ measured to high $\mathrm{J}$ values, Paper C2, Symposium on Molecular Structure and Spectra, Ohio State University, Columbus, Ohio (June 14-18, 1965).

[64] (a) Fowler, A., Terrestrial reproduction of the spectra of the tails of recent comets, Monthly Notices Roy. Astron. Soc. 70, 176-82 (190910).

(b) Fowler, A., Investigations relating to the spectra of comets, Monthly Notices Roy. Astron. Soc. 70, 484-96 (1910).

[65] Fox, R. E., and Hickam, W. M., Ionization probilbility curves for $\mathrm{CO}, \mathrm{N}_{2}, \mathrm{C}_{3} \mathrm{H}_{6}$, and $\mathrm{C}_{6} \mathrm{H}_{6}, \mathrm{~J}$. Chem. Phys. 22, 2059-63 (1954).

[66] Fraga, S., and Ransil, B. J., Studies in molecular structure. VII. Limited configuration interaction for selected first-row diatomics, J. Chem. Phys. 36, 1127-42 (1962).

[67] Fraser, P. A., A method of determining the electronic transition moment for diatomic molecules, Can. J. Phys. 32, 515-21 (1954).

[67] (a) Freund, R. S., and Klemperer, W., Radiofrequency spectrum of the $a^{3} \Pi$ state of carbon monoxide, J. Chem. Physs. 43, 2422-S (1965).

[68] Garg, S. N., New bands of the Asundi band-system of CO, Indian J. Phys. 23, 161-71 (1949).

[69] Gaydon, A. G., Dissociation Energies and Spectra of Diatomic Mlolecules, Second Edition (Chapman and IIall, London, 1953).

[70] Gaydon, A. G., and Penney, W. G., The dissociation energies of $\mathrm{CO}, \mathrm{N}_{2}, \mathrm{NO}$, and $\mathrm{CX}$, Proc. Lioy. Soc. (Lonclon), Ser. A: 133, 374-SS $(1945)$.

[71] Gerö, L., On the $A^{1} I I$ state of CO, 'Z. Physik 93, 669-75 (1935).

[72] Gerö, I.., Perturbation and predissociation in the $b^{3} \mathbf{z}^{+}$-term of the CO band spectrum, $/ 2$. l'lysik 95. $747-51(1935)$. 
[73] Gerö, L., On the $\mathrm{A}^{1} \Pi \rightarrow \mathrm{X}^{1} \Sigma^{+}$(IV pos.) carbon monoxide bands, Z. Physik 99, 52-64 (1936).

[74] Gerö, L., On the $\mathrm{A}^{1} I I \rightarrow \mathrm{X}^{1} \Sigma^{+}(\mathrm{IV}$ pos.) $\mathrm{CO}$ bands. II., Z. Physik 100, 374-88 (1936).

[75] Gerö, L., Rotational analysis of the $(1,0) b^{3} \Sigma^{+} \rightarrow$ $a^{3} \Pi$ CO band, Z. Physik 101, 311-22 (1936).

[76] Gerö, L., Rotational analysis of the $a^{3} \Pi \rightarrow \mathrm{X}^{1} \Sigma^{+}$ CO band system, Z. Physik 109, 204-9 (1938).

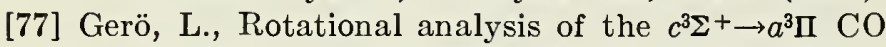
band system, Z. Physik 109, 210-15 (1938).

[78] Gerö, L., Rotational analysis of the $a^{3} \Sigma^{+} \rightarrow a^{3} \Pi$ bands of CO, Z. Physik 109, 216-22 (1938).

[79] Gerö, L., Herzberg, G., and Schmid, R., On the Cameron bands $\left({ }^{3} \Pi^{-1} \Sigma\right)$ of carbon monoxide, Phys. Rev. 52, 467-71 (1937).

[80] Gerö, L., and Lörinczi, K., Rotational analysis of the $a^{\prime 3} \Sigma^{+} \rightarrow a^{3} \Pi$ CO bands, Z. Physik 113, 449-61 (1939).

[81] Gerö, L., and Schmid, R., Completion of the term scheme of carbon monoxide. III., Z. Physik 112, 676-80 (1939).

[82] Gerö, L., and Szab6, F., On the structure of the triplet bands $\left(d^{3} \Pi \rightarrow a^{3} \Pi\right)$ of $\mathrm{CO}$, Ann. Physik 35, 597-618 (1939).

[83] Gilliam, O. R., Johnson, C. M., and Gordy, W., Microwave spectroscopy in the region from two to three millimeters, Phys. Rev. 78, 140-4 (1950).

[84] Glockler, G., The heat of dissociation of carbon monoxide, J. Chem. Phys. 18, 1517-18L (1950).

[85] Goldberg, L., and Müller, E. A., Carbon monoxide in the sun, Astrophys. J. 118, 397-411 (1953).

[86] Gordy, W., and Cowan, M. J., Precision measurements of millimeter and submillimeter wave lines of CO (abstract), Bull. Am. Phys. Soc. 2, 212-13 (1957).

[87] Hagstrum, H. D., On the dissociation energy of carbon monoxide and the heat of sublimation of carbon, Phys. Rev. 72, 947-63 (1947).

[88] Hagstrum, H. D., Reinterpretation of electron impact experiments in $\mathrm{CO}, \mathrm{N}_{2}, \mathrm{NO}$, and $\mathrm{O}_{2}$, J. Chem. Phys. 23, 1178-9L (1955).

[88] (a) Halmann, M., and Laulicht, I., Isotope effects on vibrational transition probabilities. III. Ionization of isotopic $\mathrm{H}_{2}, \mathrm{~N}_{2}, \mathrm{O}_{2}, \mathrm{CO}$ and and $\mathrm{HCl}$ molecules, J. Chem. Phys. 43, 1503-9 (1965).

[89] Hand, C. W., Short UV emission from acetylene flames, J. Chem. Phys. 36, 2521-2L (1962).

[90] Headrick, L. B., and Fox, G. W., New measurements on the fourth positive bands of carbon monoxide, Phys. Rev. 35, 1033-7 (1930).

[91] Henning, H. J., The absorption spectra of $\mathrm{CO}_{2}$ $\mathrm{CO}$, and $\mathrm{H}_{2} \mathrm{O}$ in the region of $600-900 \AA$, Ann. Physik 13, 599-620 (1932); errata, ibid 14, 856 (1932).

[92] Herman, L., and Herman, R., Emission spectrum of $\mathrm{CO}$ after atomic recombination, Compt. Rend. 225, 230-2 (1947).

[93] Herman, L., and Rakotoarijimy, D., Selective excitation of molecular emission by the metastable atoms of rare gases, J. Phys. Radium 21, 629-33 (1960).
[94] Herman, R., Remarks on the Cameron bands and their possible presence in night-sky radiation, Ann. Astrophys. 10, 42-6 (1947).

[95] Herman, R., Emission of CO in the region of the $4050 \AA$ group from nuclei of comets, Compt. Rend. 228, 1691-3 (1949).

[96] Herman, R., and Herman, L., On the triplet system of the neutral CO molecule, J. Phys. Radium (Ser. 8) 9, 160-2 (1948).

[97] Herzberg, G., On the band spectra of $\mathrm{CO}$, according to experiments with the electrodeless discharge (with a supplement on the Swan and cyanogen bands), Z. Physik 52, 815-45 (1929).

[98] Herzberg, G., The heat of dissociation of the carbon monoxide molecule and the heat of sublimation of carbon, Chem. Rev. 20, 145-67 (1937).

[99] Herzberg, G., Spectra of Diatomic Molecules, Second Edition, Seventh Printing (D. Van Nostrand Co., Inc., New York, New York, 1961).

[100] Herzberg, G. (private communication).

[101] Herzberg, G., and Hugo, T. J., Forbidden transitions in diatomic molecules: IV. The $a^{\prime 3} \Sigma^{+\leftarrow} \mathrm{X}^{1} \Sigma^{+}$and $e^{3} \Sigma^{-\leftarrow} \mathrm{X}^{1} \Sigma^{+}$absorption bands of $\mathrm{CO}, \mathrm{Can} . \mathrm{J}$. Phys. 33, 757-72 (1955).

[102] Herzberg, G., and Rao, K. N., Rotation-vibration spectra of diatomic and simple polyatomic molecules with long absorbing paths: II. The spectrum of CO below $1.2 \mu$, J. Chem. Phys. 1\%, 1099-1102 (1949).

[102] (a) Hesser, J. E., and Dressler, K., Absolute transition probabilities in the ultraviolet spectrum of CO, Astrophys. J. 142, 389-90L (1965).

(b) Hesser, J. E., and Dressler, K., Transition probabilities in $\mathrm{NO}, \mathrm{NO}^{+}$, and $\mathrm{CO}$, Paper at Symposium on Molecular Structure and Spectroscopy, The Ohio State University, Columbus (June 14-18, 1965).

[103] Hopfield, J. J., Absorption spectra in the extreme ultra-violet (abstract), Phys. Rev. 29, 356 (1927).

[104] Hopfield, J. J., and Birge, R. T., Ultra-violet absorption and emission spectra of carbon monoxide (abstract), Phys. Rev. 29, 922 (1927).

[105] Howell, H. G., Dissociation energy of carbon monoxide, Nature 163, 773-4 (1949).

[106] Huber, K. P., The Rydberg series in the absorption spectrum of the NO molecule, Helv. Phys. Acta 34, 929-53 (1961).

[107] Huffman, R. E., Larrabee, J. C., and Tanaka, Y., Absorption coefficients of carbon monoxide in the 1006-600 ^ wavelength region, J. Chem. Phys. 40, 2261-9 (1964).

[108] Hulthén, E., On the band spectrum of carbon monoxide, Ann. Physik \%1, 41-9 (1923).

[109] (a) Huo, W., Self-consistent field wave functions for the ground state of $\mathrm{CO}$ and $\mathrm{BF}(\mathrm{Ph} . \mathrm{D}$. Thesis), Department of Chemistry, University of Chicago (1964).

(b) Huo, W. M., Electronic structure of $\mathrm{CO}$ and BF, J. Chem. Phys. 43, 62£-47 (1965).

[110] Hurley, A. C., Electronic structure and binding energy of carbon monoxide, Rev. Mod. Phys. 32. 400-11 (1960). 
[111] Hurley, A. C., and Maslen, V. W., Potential curves for doubly positive diatomic ions, J. Chem. Phys. 34, 1919-25 (1961).

[112] Ittmann, G. P., On the theory of perturbations in band spectra, Z. Physik $71,616-26$ (1931).

[113] Jarmain, W. R., Ebisuzaki, R., and Nicholls, R. W., Franck-Condon factors and $r$-centroids for some bands of the $\mathrm{CO}$ fourth positive $\left(\mathrm{A}^{1} \Pi-\mathrm{X}^{1} \Sigma\right)$ band system, Can. J. Phys. 38, 510-12 (1960).

[114] Jarmain, W. R., Fraser, P. A., and Nicholls, R. W., Vibrational transition probabilities of diatomic molecules; collected results. III. $\mathrm{N}_{2}, \mathrm{NO}, \mathrm{C}_{2}$, $\mathrm{O}_{2}{ }^{+}, \mathrm{OH}, \mathrm{CO}, \mathrm{CO}^{+}$, Astrophys. J. 122, 55-61 (1955).

115] (a) Jasse, O., On the structure of the 4511 and 4123 bands of the CO spectrum, Compt. Rend. 182, 692-4 (1926).

(b) Jasse, O., On the 4511 and 4123 bands of the carbon monoxide spectrum, Rev. Opt. 5, 450-61 (1926).

[116] Jevons, W., On the origin of certain spectral lines hitherto attributed to oxygen, Phil. Mag. 47, 586-90 (1924).

[117] Jevons, W., Report on Band Spectra of Diatomic Molecules (The Physical Society, London, 1932).

[118] Johnson, R. C., Further spectra associated with carbon, Proc. Roy. Soc. (London), Ser. A: 108, 343-55 (1925).

[119] Johnson, R. C., The spectra of the neutral carbon monoxide molecule, Nature 117, 376-7L (1926).

[120] Johnson, R. C., An Introduction to Molecular Spectra (Methuen and Co., Ltd., London, 1949).

[121] Johnson, R. C., and Asundi, R. K., A new band system of carbon monoxide $\left(3^{1} \mathrm{~S} \rightarrow 2^{1} \mathrm{P}\right)$, with remarks on the Ångstrom band system, Proc. Roy. Soc. (London), Ser. A: 123, 560-74 (1929).

[122] Jones, G., and Gordy. W., Extension of submillimeter wave spectroscopy below a half-millimeter wavelength, Phys. Rev. 135A, A295-7 (1964).

[123] Kamper, R. A., Lea, K. R., and Lustig, C. D., Hyperfine structure and nuclear electric quadruple moment of ${ }^{17} \mathrm{O}$, Proc. Phys. Soc. (London) By0, 897-9L (1957).

[124] Kaneko, Y., Ionization efficiency curves for $\mathrm{A}^{+}, \mathrm{Kr}^{+}$, $\mathrm{N}_{2}{ }^{+}$, and $\mathrm{CO}^{+}$by electron impact, J. Phys. Soc. Japan 16, 1587-93 (1961).

[125] Kaplan, J., A new system of bands in carbon monoxide, Phys. Rev. 35, 1298L (1930).

[126] Kaplan, J., The quenching of mercury resonance radiation by nitrogen and carbon monoxide (abstract), Phys. Rev. 36, 788 (1930).

[127] (a) Kayser, H. G. J., Handbuch der Spectroscopie, Vol. 5, (S. Hirzel, Leipzig, 1910).

(b) Kayser, H. G. J., and Konen, H., Handbuch der Spectroscopie, Vol. 7 (S. Hirzel, Lcipzig, 1924-34).

[128] Kemble, E. C., Mulliken, R. S., and Crawford, F. H., The Zeeman effect in the Angstrom CO bands, Phys. Rev. 30, 438-57 (1927).

[129] Kiser, R. W., Tables of ionization potentials, TID6142 (June 20, 1960).

[130] Knauss, H. P., CO bands in the region $\lambda 2220$ to $\lambda$ 3300 (abstract), Ph s. Rev. 37, 471-2 (1931).
[131] Knauss, H. P., and Cotton, J. C., Ultraviolet bands of $\mathrm{CO}$ in the electrodeless ring discharge, Phys. IRev. 38, 1190-4 (1931).

[132] Knight, II. T., and Rink, J. P., Heat of sublimation of carbon and the dissociation energy of $\mathrm{CO}$ by X-ray densitometry of shock waves, J. Chem. Phys. 29, 449-50L, (1958).

[133] Kolesnikov, V. N., and Leskov, L. V., Optical transition probabilities for atoms and diatomic molecules, Uspekhi. Fiz. Nauk 65 (1), 2-38 (1958); translated as pp. 347-90 in Meroz, J. (Ed.), Optical Transition Probabilities, A Collection of Russian Articles 1924-1960 (Published for NSF by Israel Program for Scientific Translations, Jerusalem, 1962).

[134] Kopelman, R., and Klemperer, W., Dipole moments of excited electronic states: Possible values for $\mathrm{CO}$ and $\mathrm{CO}^{+}$, J. Chem. Phys. 36, 1693-4L (1962).

[135] Kovács, I., Determination of the rotation and vibration constants of diatomic molecular terms on the basis of perturbations, Math. Naturw. Anz. Ungar. Akad. Wiss. 56, 126-57 (1937).

[136] (a) Kovács, I., Intensity formulae for ${ }^{3} \Delta-{ }^{3} \Pi$ bands, Nuovo Cimento 29, 1089-97 (1963).

(b) Kovács, I., and Torös, R., The intensity distribution of the triplet bands of the $\mathrm{CO}$ molecule, Acta Phys. Acad. Sci. Hung. 18, 101-6 (1965).

[137] (a) Kovács, I., Some new results in the spectroscopic investigation of diatomic molecules, Acta Phys. Acad. Sci. Hung. 17, 67-74 (1964).

(b) Kovács, I., The rotational structure of the $d^{3} \Delta$ state of the $\mathrm{CO}$ molecule, Acta Phys. Acad. Sci. Hung. 18, 107-10 (1965).

[138] Krupenie, P. H., and Weissman, S., Potential energy curves for $\mathrm{CO}$ and $\mathrm{CO}^{+}$, J. Chem. Phys. 43, 1529-34 (1965).

[139] Lagemann, R. T., Niclsen, A. H., and Dickey, F. P., The infra-red spectrum and molecular constants of $\mathrm{C}^{12} \mathrm{O}^{16}$ and $\mathrm{C}^{13} \mathrm{O}^{16}$, Phys. Rev. 72, 284-\$8 (1947).

[140] (a) Lagerqvist, A., Nilheden, G., and Barrow, R. F., The near ultra-violet band system (D-X) of silicon monosulphide, Proc. Phys. Soc. (Lonclon) A 65, 419-32 (1952).

(b) Lagerqvist, A., and Uhler, U., The ultra-violet band-system of silicon monoxide, Arkiv Fysik 6, 95-111 (1952).

[141] Lassettre, E. N. (private communication).

[142] Lassettre, E. N., and Silverman, S. M., Inelastic collision eross sections of earbon monoxide, J. Chem. Phys. 10, 1256-61 (1961).

[143] Lawrence, G. M., Lifetimes and transition probabilities in $\mathrm{CO}^{+}$, J. Quant. Spectry. Radiative Transfer 5, 359-67 (1965).

[144] Lefebvre-Brion, I., Moser, C. M., and Nesbet, R. I., The $\mathbf{1}^{+}$exeited states of carbon monoxide, J. Chem. Phys. 33, 931-2L (1960).

[145] Lefebvre-Brion, H., Moser, C. M., and Nesbet, R. I゙., A calculation of the potent ial energy curves for some electronic states of earbon monoxicle, J. Chem. Phys. 34, 1950-7 (1961).

[146] Lefohvre-13rion, H., Moser, C., and Veshet, R. К.. Ultraviolet spectra of carbon monoxide, J. Chem. Phys. 35, 1702-7 (1961). 
[147] Lefebvre-Brion, H., Moser, C. M., and Nesbet, R. K., Rydberg levels in carbon monoxide, J. Mol. Spectry. 13, 418-29 (1964).

[148] Lefebvre-Brion, H., Moser, C., Nesbet, R. K., and Yamazaki, M., Electric-field gradient at the oxygen nucleus in $\mathrm{CO}$ and the dipole moment of CO, J. Chem. Phys. 38, 2311-13L (1963).

[149] Leifson, S. W., Absorption spectra of some gases and vapors in the Schumann region, Astrophys. J. 63, 73-89 (1926).

[150] Leskov, L. V., On the method of quantitative gas analysis using electron-vibrational spectra of diatomic molecules, Opt. i Spektroskopiya 4, 168-79 (1958). (P.A. 62, 12858).

[151] Lindholm, E., Ionization and fragmentation of $\mathrm{CO}$ by bombardment with atomic ions. Dissociation energy of CO. Heat of sublimation of carbon, Arkiv Fysik 8, 433-40 (1954).

[152] Linnett, J. W., Binding in some diatomic molecules, J. Chem. Soc., 275-87 (1956).

[153] Lofthus, A., The Molecular Spectrum of Nitrogen, Spectroscopic Report Number 2, Department of Physics, University of Oslo, Blindern, Norway (Dec. 1960).

[154] Long, L. H., and Walsh, A. D., Remarks on the structure of carbon monoxide, Trans. Faraday Soc. 43, 342-51 (1947).

[155] Lowry, E. F., The infrared absorption spectrum of carbon monoxide, J. Opt. Soc. Am. 8, 647-58 (1924).

[156] McCay, M. S., Structure of the (0,4) Angstrom band of $\mathrm{CO}$ for high rotational states (abstract), Phys. Rev. 59, 911 (1941).

[157] (a) McCulloh, K. E., A study of the electronic band spectrum of the carbon-13 modification of carbon monoxide (Ph. D. Thesis), Department of Chemistry and Chemical Engineering, State University of Iowa (1951).

(b) MeCulloh, K. E., and Glockler, G., The electronic emission spectrum of $\mathrm{C}^{13} \mathrm{O}^{16}$, Phys. Rev. 89, 145-7 (1953).

[158] McLennan, J. C., Smith, H. G., and Peters, C. S., The infra-red spectra of certain elements, Trans. Roy. Coc. Can. Sect. III 19, 39-50 (1925).

[159] Merryman, P., Moser, C. M., and Nesbet, R. K., Lower excited electronic states of carbon monoxide, J. Chem. Phys. 32, 631-2L (1960).

[160] Merton, T. R., and Johnson, R. C., On spectra associated with carbon, Proc. Roy. Soc. (London) Ser. A: 103, 383-95 (1923).

[161] Migeotte, M., Neven, L., and Swensson, J., The solar spectrum from 2.8 to 23.7 microns, Part I: Photometric Atlas, from Mem. Soc. Roy. Sci. Liège, Special Volume No. 1 (1956); Part II: Measures and Identifications, from Mem. Soc. Roy. Sci. Liège, Special Volume No. 2 (1957).

[162] Mills, I. M., and Thompson, H. W., The fundamental vibration-rotation bands of ${ }^{13} \mathrm{C}^{16} \mathrm{O}$, and ${ }^{12} \mathrm{C}^{18} \mathrm{O}$, Trans. Faraday Soc. $\mathbf{4 9 ,} 224-7$ (1953).

[163] Mizushima, M., Some corrections to the second order Stark effect of linear molecules, pp. 1162-84, in Adv. Mol. Spectry. (Proceedings of the IVth Intern. Meeting on Molecular Spectroscopy,
Bologna and Rome, 1959), Vol. 3, Mangini, A. (ed.) (The Macmillan Co., New York, 1962).

[164] Moffitt, W., The electronic structures of carbon monoxide and carbon dioxide, Proc. Roy. Soc. (London), Ser. A: 196, 524-39 (1949).

[165] Mohler, O. C., A table of solar spectrum wavelengths $11984 \AA$ to $25578 \AA$, (The University of Michigan Press, Ann Arbor, 1955). (See pp. 65-83, CO solar IR lines.)

[166] Moore, C. E., Atomic Energy Levels, NBS Circ. 467, Vol. I, (1949).

[167] Mulliken, R. S., The assignment of quantum numbers for electrons in molecules. II. Correlation of molecular and atomic electron states, Phys. Rev. 32, 761-72 (1928).

[168] Mulliken, R. S., The interpretation of band spectra: Part III. Electron quantum numbers and states of molecules and their atoms, Rev. Mod. Phys. 4, $1-86$ (1932).

[169] Mulliken, R. S., The halogen molecules and their spectra. $J-J$ like coupling. Molecular ionization potentials, Phys. Rev. 46, 549-71 (1934).

[170] Mulliken, R. S., Electronic population analysis on LCAO-MO molecular wave functions. I., J. Chem. Phys. 23, 1833-40 (1955).

[171] Mulliken, R. S., The lower excited states of some simple molecules, Can. J. Chem. 36, 10-23 (1958).

[172] Mulliken, R. S., Self-consistent field atomic and molecular orbitals and their approximations as linear combinations of Slater-type orbitals, Rev. Mod. Phys. 32, 232-8 (1960).

[173] Nesbet, R. K., Electronic structure of $\mathrm{N}_{2}, \mathrm{CO}$, and BF, J. Chem. Phys. 40, 3619-33 (1964).

[174] Nicholls, R. W., Molecular band intensities and their interpretation, pp. 302-23 in Armstrong, E. B., and Dalgarno, A. (eds.), The Airglow and the Aurorae (Pergamon Press, London, 1956).

[175] Nicholls, R. W., Studies upon transition probabilities and molecular excitation, Ann. Geophys. 14, 208-24 (1958).

[176] Nicholls, R. W., Interpretation of intensity distributions in the $\mathrm{N}_{2}$ Lyman-Birge-Hopfield and $\mathrm{CO}$ fourth positive band systems, Nature 186, 958-9 (1960).

[177] Nicholls, R. W., Franck-Condon factors and $r$ centroids to high vibrational quantum numbers for three band systems of $\mathrm{CO}^{+}$and absolute band strengths for the comet-tail system, Can. J. Phys. 40, 1772-83 (1962).

[178] Nicholls, R. W., Laboratory astrophysics, J. Quant. Spectry. Radiat. Transfer 2, 433-49 (1962).

[179] Nicholls, R. W., Fraser, P. A., Jarmain, W. R., and MeEachran, R. P., Vibrational transition probabilities of diatomic molecules: Collected results. IV. $\mathrm{BeO}, \mathrm{BO}, \mathrm{CH}^{+}, \mathrm{CO}, \mathrm{NO}, \mathrm{SH}, \mathrm{O}_{2}, \mathrm{O}_{2}{ }^{+}$, Astrophys. J. 131, 399-406 (1960).

[180] Nicholls, R. W., and Jarmain, W. R., r-centroids: Average internuclear separation associated with molecular bands, Proc. Phys. Soc. (London) A69, 253-64 (1956).

[181] Nicholls, R. W., and Stewart, A. L., Allowed Transitions, pp. 47-78 in Bates, D. R. (ed.), Atomic and Molecular Processes (Academic Press, New York, 1962). 
[182] Onaka, R., Perturbation at $v=6$ of the $A^{\prime} I I$ state of CO, J. Chem. Phys. 26, 1763-4 (1957).

[183] Ortenberg, F. S., Calculation of Franck-Condon factors for the $\mathrm{NO}, \mathrm{C}_{2}$, and $\mathrm{CO}$ band systems, Opt. Spectry (USSR) 16, 398-400 (1964).

[184] Palik, E. D., and Rao, K. N., Pure rotational spectra of $\mathrm{CO}, \mathrm{NO}$, and $\mathrm{N}_{2} \mathrm{O}$ between 100 and 600 microns, J. Chem. Phys. 25, 1174-6 (1956).

[185] Patel, C. K. N., and Kerl, R. J., Laser oscillations on $\mathrm{X}^{1} \Sigma^{+}$vibrational-rotational transitions of $\mathrm{CO}$, Appl. Phys. Letters 5, 81-3 (1964).

[186] Pearse, R. W. B., The spectrum of the night sky: Some problems in the identifications, Intern. Conf. (July, 1947) Gassiot Comm., Roy. Soc. London, 12-15 (1948). (C. A. 44, 5699f).

[187] Pearse, R. W. B., and Gaydon, A. G., The Identification of Molecular Spectra, Third Edition (John Wiley \& Sons, Inc., New York, N.Y., 1963).

[188] Pillow, M. E., and Rowlatt, A. L., Intensities in the triplet system of CO bands, Proc. Phys. Soc. (London) A75, 162L (1960).

[189] (a) Pluvinel, A. B., and Baldet, F., On the spectrum of the comet 1908c (Morehouse), Compt. Rend. 148, 759-62 (1909).

(b) Pluvinel, A. B., and Baldet, F., Spectrum of comet Morehouse (1908c), Astrophys. J. 34, 89-104 (1911).

[190] Plyler, E. K., Allen, Jr., H. C., and Tidwell, E. D., Emission spectrum of carbon monoxide from 2.3 to 2.5 microns, J. Res. NBS 61 (Phys. and Chem.) No. 1, 53-6 (1958).

[191] Plyler, E. K., Benedict, W. S., and Silverman, S., Precise measurements in the infrared spectrum of carbon monoxide, J. Chem. Phys. 20, 175-84 (1952).

[192] Plyler, E. K., Blaine, L. R., and Conner, W. S., Velocity of light from the molecular constants of carbon monoxide, J. Opt. Soc. Am. 45, 102-6 (1955).

[193] Plyler, E. K., Blaine, L. R., and Tidwell, E. D., Infrared absorption and emission spectra of carbon monoxide in the region from 4 to 6 microns, J. Res. NBS 55 No. 4, 183-9 (1955).

[194] Plyler, E. K., Danti, A., Blaine, L., and Tidwell, E. D., Vibration-rotation structure in absorption bands for the calibration of spectrometers from 2 to 16 microns, J. Res. NBS $64 \mathbf{A}$ (Phys. and Chem.) No. 1, 29-48 (1960).

[195] Popova, T. N., and Sashchenko, N. MI., FranckCondon factors for some vibrational transisitions in the fourth positive system of CO, Opt. Spectry. (USSR) 12, 447-8 (1962).

[196] Rank, D. H., Eastman, D. P., Rao, B. S., and Wiggins, T. A., Highly precise wavelengths in the infrared. II. $\mathrm{HCN}, \mathrm{N}_{2} \mathrm{O}$, and $\mathrm{CO}$, J. Opt. Soc. Am. 51, 929-36 (1961).

[197] Rank, D. H., Guenther, A. H., Saksena, G. D., Shearer, J. N., and Wiggins, T. A., Tertiary interferometric wavelength standards from ineasurements on lines of the 2-0 balld of carbon monoxide and derived wavelength standards for some lines of the 1-0 band of carbon monoxide. The velocity of light derived from a band spectrum method. IV, J. Opt. Soc. Am. 47, 686-9 (1957).

[198] Rank, D. H., Skorinko, G., Eastman, J). P., and Wiggins, T. A., Highly precise wavelengths in the infrared, J. Mol. Spectry. 4, 518-33 (1960).

[199] Ransil, B. J., Quadrupole coupling constant, dipole moment, and electron population of $\mathrm{CO}, \mathrm{J}$. Chem. Phys. 30, 1113-4L (1959).

[200] Ransil, B. J., Studies in molecular structure. II. LCAO-MO-SCF wave functions for selected first-row diatomic molecules, Rev. Mod. Phys. 32, 245-54 (1960).

[201] Rao, K. N., Structure of the Cameron bands of CO, Astrophys. J., 110, 304-11 (1949).

[202] (a) Rao, K. N., The band spectrum of $\mathrm{CO}^{+}$. I. First negative system $\left(\mathrm{B}^{2} \Sigma-\mathrm{X}^{2} \Sigma\right)$, Astrophys. J. 111, 50-9 (1950).

(b) Rao, IK. N., The band spectrum of $\mathrm{CO}^{+}$. II. Comet-tail system $\left(\mathrm{A}^{2} \Pi-\mathrm{X}^{2} \Sigma\right)$, Astrophys. J. 111, 306-13 (1950).

[203] (a) Rao, K. N., Problem of wavelength calibrations in the infrared, presented to the Triple Commission for Spectroscopy (June, 1963).

(b) Rao, K. N., Wavelength standards in the infrared, presented to the Triple Commission for Spectroscopy (Sept., 1964).

[204] Rao, K. N., deVore, R. V., and Plyler, E. K., Wavelength calibrations in the far infrared (30 to 1000 microns), J. Res. NBS 6rA (Phys. and Chem.) No. 4, 351-8 (1963).

[205] Rao, K. N., and Sarma, I. S., Band spectrum of $\mathrm{CO}^{+}$. The Baldet-Johnson system ( $\left.\mathrm{B}^{2} \Sigma-\mathrm{A}^{2} \Pi\right)$, Mem. Soc. Roy. Sci. Liege 13, 181-6 (1953).

[206] (a) Rasetti, F., Raman effect in gases, Nature 123, 205L (1929).

(b) Rasetti, F., On the Raman effect in diatomic molecules, Nuovo Cimento 6, 356-70 (1929).

[207] Rasetti, F., On the Raman effect in diatomic gases, Proc. Nat. Acad. Sci. U.S. 15, 234-37 (1929).

[208] (a) Rave, W., Stark effect in the molecular spectrum of nitrogen, carbon monoxide, and hydrogen, Z. Physik 94, 72-84 (1935).

(b) Rave, W., Correction to the work "The Stark effect in the molecular spectra of nitrogen, carbon monoxide, and hydrogen," Z. Physik 96, 276-7 (1935).

[209] (a) Read, D. N., The rotational and vibrational analysis of the fourth positive bands of carbon monoxide (Ph. D. Thesis), Department of Physics, Princeton University (1934).

(b) Read, D. N., Rotational and vibrational structure of the fourth positive bancls of carbon monoxicke, Physs. Rev. 46. 571-5 (1931).

[210] Robinson, 1)., Ph.D. 'Thesis, University of Vestern Ontario (1957).

[211] Robinson, D., and Nicholls, R. W., Intensity mensurements on the $\mathrm{O}_{2}{ }^{+}$second negative, $\mathrm{CO}$ Angstrom and third positive, and $\mathrm{NO} \gamma$ and $\beta$ molecular band systems, Proc. Physs. Soc. (Lonclon) A 71, 957-64 (195\$).

[212] Robinson, 1)., and Nicholls, R. W., Intensity mcasurements on the $\mathrm{CO}^{+}$connet tail, and the $13 \mathrm{O}$ 
$\alpha$ and $\beta$ molecular bands systems, Proc. Phys. Soc. (London) A75, 817-25 (1960).

[213] Rosen, B., et al. (ed.), Constantes sélectionées; atlas des longeurs d'onde caracteristiques des bandes d'emission et d'absorption des molécules diatomiques, (Herman d'epositoires, Paris, 1952).

[214] Rosenblum, B., and Nethercot, Jr., A. H., Quadrupole coupling constant and molecular structure of $\mathrm{CO}^{17}$, J. Chem. Phys. 2\%, 828-9L (1957).

[215] Rosenblum, B., Nethercot, A. H., and Townes, C. H., Isotopic mass ratios, magnetic moments, and sign of the electric dipole moment in carbon monoxide, Phys. Rev. 109, 400-12 (1958).

[216] Rosenthal, J., and Jenkins, F. A., Perturbations in band spectra, II., Proc. Nat. Acad. Sci. U. S. 15, 896-902 (1929).

[217] Sahni, R. C., The structure of carbon monoxide, Trans. Faraday Soc. 49, 1246-53 (1953).

[218] St. Pierre, A. G., Highly precise measurements of the 2-0, 3-1, and 4-2 bands of carbon monoxide (M.S. Thesis), Department of Physics, Pennsylvania State University, (March, 1964).

[219] (a) Sato, K., A study of the spectrum of carbon monoxide by a high-frequency discharge, Bunko Kenkyu (Japan) 8, 145-9 (1960).

(b) Sato, K., Studies on the spectrum of carbon mono-oxide by high frequen cy $33 \mathrm{Mc}$ discharge, Paper D 306-1, Proc. Intern. Symp. on Mol. Structure and Spectry., First, (Sci. Council of Japan, 1962).

[220] Schlapp, R., Intensities in singlet-triplet bands of diatomic molecules, Phys. Rev. 39, 806-15 (1932).

[221] Schmid, R., On a predissociation limit of $\mathrm{CO}$ at 11.6 V, Physik. Z. 37, 55-6 (1936).

[222] Schmid, R., and Gerö, L., On the rotational analysis of the ${ }^{2} \Sigma \rightarrow^{2} \Sigma$ and ${ }^{2} \Pi \rightarrow^{2} \Sigma$ bands of $\mathrm{CO}^{+}, \mathrm{Z}$. Physik 86, 297-313 (1933).

[223] Schmid, R., and Gerö, L., On the Zeeman effect of the comet tail bands, Z. Physik 86, 314-20 (1933).

[224] (a) Schmid, R., and Gerö, L., On the $\mathrm{B}^{1} \Sigma \rightarrow \mathrm{A}^{1} \Pi$ and $\mathrm{C}^{1} \Sigma \rightarrow \mathrm{A}^{1} \Pi$ bands of $\mathrm{CO}, \mathrm{Z}$. Physik 93, 656-68 (1935).

(b) Schmid, R., and Gerö, L., Predissociation of the $\mathrm{C}^{1} \Sigma$ state of CO, Z. Physik 96, 546-50 (1935).

[225] Schmid, R., and Gerö, L., Zeeman effect of the perturbations of the $\mathrm{A}^{1} \Pi$ state in $\mathrm{CO}, \mathrm{Z}$. Physik 94, 386-96 (1935).

[226] Schmid, R., and Gerö, L., On the structure of the 5B-bands of the CO spectrum, Z. Physik 96, 198-202 (1935).

[227] Schmid, R., and Gerö, L., Predissociation in the $\mathrm{A}^{1}$ II state of $\mathrm{CO}$; Dissociation energy of $\mathrm{CO}$ at 6.9 volts, Z. Physik 99, 281-4 (1936).

[228] Schmid, R., and Gerö, L., On the rotational constants of the IV. pos. CO bands, Z. Physik 101, 343-51 (1936).

[229] Schmid, R., and Gerö, L., The energy levels of the CO molecule, Math. Naturw. Anz. Ungar. Akad. Wiss. 55, 691-765 (1937).

[230] Schmid, R., and Gerö, L., Rotational analysis of the "3A" bands of CO, Nature 139, 928L (1937).

[231] Schmid, R., and Gerö, L., Structure of a new system of CO bands, Nature 140, 508L (1937).
[232] Schmid, R., and Gerö, L., Intensive emission photographs of the $a^{3} \Pi \rightarrow \mathrm{X}^{1} \Sigma$ (Cameron) intercombination bands of carbon monoxide with high dispersion, Naturwissenschaften 25, 90 (1937).

[233] Schmid, R., and Gerö, L., Red-degraded bands of $\mathrm{CO}$ in the region of $2670-3310 \AA$, Naturwissenschaften 25, 90 (1937).

[234] (a) Schmid, R., and Gerö, L., On completion of the term scheme of carbon monoxide. I. Vibrational terms and rotational constants of the $a^{\prime 3} \Sigma^{+}$state, Z. Physik 105, 36-44 (1937).

(b) Schmid, R., and Gerö, L., On completion of the term scheme for CO. II., Z. Physik 106, 205-11 (1937).

[235] Schmid, R., and Gerö, L., Dissociation energy of carbon monoxide, Z. Physik. Chemie B 36, 105-28 (1937).

[236] (a) Schmid, R. F., Zeeman effect of the third positive carbon bands, Phys. Rev. 39, 538-9L (1932).

(b) Schmid, R., Zeeman effect on the triplet bands. Influence of a magnetic field on the lines of the third positive CO bands, Z. Physik 89, 701-7 (1934).

[237] Schmid, R. F., Rotational analysis of the firstnegative bands of the $\mathrm{CO}^{+}$molecule, Phys. Rev. 42, 182-8 (1932).

[237] (a) Schwenker, R. P., Experimental oscillator strengths of $\mathrm{CO}$ and $\mathrm{CO}^{+}$, J. Chem. Phys. 42, 1895-8 (1965).

[238] Silverman, S. M., and Lassettre, E. N., Intensity distribution for the $\mathrm{X}^{1} \Sigma^{+} \rightarrow \mathrm{A}^{1} \Pi$ transition in carbon monoxide excited by electron impact, $\mathrm{J}$. Chem. Phys. 41, 3727-9 (1964).

[238] (a) Simmons, J. D., Bass, A. M., and Tilford, S. G., High resolution absorption spectrum of the carbon monoxide fourth positive system, Paper at Symposium on Molecular Structure and Spectroscopy, The Ohio State University, Columbus (June 14-18, 1965).

[239] Singh, N. L., and Jain, D. C., Relative intensities in the triplet system of $\mathrm{CO}$ bands, Proc. Phys. Soc. (London) A 77, 817-18L (1961).

[240] Singh, N. L., and Jain, D. C., Franck-Condon factors and $r$-centroids for the triplet band system of $\mathrm{CO}$ molecule, Proc. Phys. Soc. (London) A 78, 399-403 (1961).

[241] Snow, C. P., and Rideal, E. K., Infra-red investigations of molecular structure: Part III. The molecule of carbon monoxide, Proc. Roy. Soc. (London) Ser. A: 125, 462-83 (1929).

[242] Soshnikov, V. N., Absolute intensity of electron transitions in diatomic molecules, Soviet Phys.Usp. 4, 425-40 (1961).

[243] Spindler, R. J., and Wentink, Jr., T., Franck-Condon factors and electronic transition moment of the $\mathrm{CO}^{+}$comet-tail system, Technical Memorandum, RAD-TM-63-55, Research and Advanced Development Division, AVCO Corporation, Wilmington, Mass. (31 July 1963).

[244] Stepanov, B. I., Investigation of the interaction of various components of level ${ }^{3} \Sigma$ with level ${ }^{1} \Pi, J$. Phys. U.R.S.S. 2, 81-8 (1940). 
[245] Stepanov, B. I., The shape of perturbation curves for the intersection of levels ${ }^{3} \Sigma$ and ${ }^{3} I I$ of the diatomic molecule, J. Phys. U.R.S.S. 2, 89-95 (1940).

[246] Stepanov, B. I., On the calculation of constants of the level $b^{3} \Sigma$ in molecule CO, J. Phys. U.R.S.S. 2, 197-204 (1940).

[247] Stepanov, B. I., On the interaction of the level $b^{3} \Sigma$ with the high vibrational levels of the triplet state $a^{\prime 3} \Sigma$ in the molecule CO, J. Phys. U.R.S.S. 2, 205-12 (1940).

[248] Steubing, W., New studies in an electric field, Physik. Z. 26, 915-19 (1925).

[249] Stevenson, M. J., and Townes, C. H., Quadrupole moment of $\mathrm{O}^{17}$, Phys. Rev. 107, 635-7L (1957).

[250] Stewart, E. T., Wave-mechanical calculations on atoms and small molecules, Ann. Rept. Progr. Chem. (Chem. Soc. London) 58, 7-28 (1962).

[251] Sun, H., and Weissler, G. L., Absorption cross sections of carbon dioxide and carbon monoxide in the vacuum ultraviolet, J. Chem. Phys. 23, 1625-8 (1955).

[252] Svensson, B., Attempt to experimentally detect the Stark effect in band spectra, Z. Physik $71,450-2$ (1931).

[253] Takamine, T., Tanaka, Y., and Iwata, M., On the first ionization potential of CO, Sci. Pap. Inst. Phys. Chem. Res. (Tokyo) 40, 371-8 (1943).

[254] Tanaka, Y., CO absorption spectra in the extreme ultraviolet, Sci. Pap. Inst. $P$ ys. Chem. Res. (Tokyo) 39, 447-55 (1942).

[255] Tanaka, Y., .ursa, A. S., and LeBlanc, F., Absorption spectrum of $\mathrm{CO}$ in the vacuum ultraviolet region, J. Chem. Phys. 26, 862-6 (1957).

[256] Tawde, N. R., and Patil, B. S., The evaluation of the electronic transition moment for the $b^{3} \Sigma^{+}-a^{3} \Pi$ system of CO, Indian J. Phys. 33, 505-10 (1959).

[257] Tilford, S. G., Vanderslice, J. T., and Wilkinson, P. G., High resolution vacuum ultraviolet absorption spectrum of the $\mathrm{E}^{1} \mathrm{II} \leftarrow \mathrm{X}^{1} \Sigma^{+}$transition in $\mathrm{CO}$, Can. J. Phys. 43, 450-6 (1965).

[258] Tobias, I., Fallon, R. J., and Vanderslice, J. T., Potential energy curves for CO, J. Chem. Phys. 33, 1638-40 (1960).

[259] Toennies, J. P., and Greene, E. F., Dissociation energies of carbon monoxide and nitrogen from reflected shock wave studies, J. Chem. Phys. 26, 655-62 (1957).

[260] Tschulanovsky, V. M., Two new electron levels of the molecule CO, J. Phys. U.R.S.S. 1, 341-6 (1939).

[261] Tschulanowsky, W. M., and Gassilewitsch, S. I., An investigation of the spectra of $\mathrm{N}_{2}$ and $\mathrm{CO}$ in the Lyman region, Phys. Z. Sowjet. 1\%, 83-8 (1937).

[262] Tschulanowsky, W. M., and Stepanow, B. I., Investigation of the fourth positive band group of the $\mathrm{CO}$ molecule in the Schumann region, Phys. $\mathrm{Z}$. Sowjet. 10, 292-314 (1936).

[263] Vaughen, A. L., Mass spectrograph analyses, and critical potentials for the production of ions by electron impact, in nitrogen and carbon monoxide, Phys. Rev. 38, 1687-95 (1931).

[264] Wacks, M. E., Franck-Condon factors for the ionization of $\mathrm{CO}$, NO, and $\mathrm{O}_{2}, \mathrm{~J}$. Chem. Phys. 41, 930-6 (1964).

[265] Wahl, C., and Huo, W., Accurate LCSTO-MO-SCF calculations for diatomic molecules of first row, Paper at Symposium on Molecular Structure and Spectroscopy, The Ohio State University, Columbus, (June 10-14, 1963).

[266] Wallace, L., Band head wavelengths of $\mathrm{C}_{2}, \mathrm{CH}, \mathrm{CN}$, $\mathrm{CO}, \mathrm{NH}, \mathrm{NO}, \mathrm{O}_{2}$, and $\mathrm{OH}$, and their ions, Astrophys. J. Suppl. 68 (1962).

[267] Watanabe, K., Ionization potentials of some molecules, J. Chem. Phys. 26, 542-7 (1957).

[268] Watson, W. W., Zeeman effect and perturbations in the CO Angstrom and the $\mathrm{N}_{2}^{+}$bands, Phys. Rer. 41, 378L (1932).

[269] Watson, W. W., Zeeman effect of perturbed terms in the CO Angstrom bands, Phys. Rev. 42, 509-17 (1932).

[270] Weissler, G. L., Samson, J.A.R., and Ogawa, M., Photoionization analysis by mass spectroscopy, J. Opt. Soc. Am. 49, 338-49 (1959).

[271] Weissman, S., Vanderslice, J. T., and Battino, R., On the recalculation of the potential energy curves for the ground states of $\mathrm{I}_{2}$ and $\mathrm{H}_{2}, \mathrm{~J}$. Chem. Phys. 39, 2226-8 (1963).

[272] Whitcomb, S. E., and Lagemann, R. T., The infrared spectrum and molecular constants of carbon monoxide, Phys. Rev. 55, 181-3 (1939).

[273] Wiggins, T. A. (private communication).

[274] Wilkinson, P. G., Molecular spectra in the vacuum ultraviolet, J. Mol. Spectry. 6, 1-57 (1961).

[275] Wilkinson, P. G., Diatomic molecules of astrophysical interest: Ionization potentials and dissociation energies, Astrophys. J. 138, 778-800 (1963).

[276] Wilkinson, P. G., and Tilford, S. (private communication).

[277] Wolter, P., On the ultraviolet bands of the CO spectrum, Z. Wiss. Phot. 9, 361-S7 (1911).

[278] Woods, L. H., Note on the spin doubling in the doublet sigma states of $\mathrm{CO}^{+}$, Phys. Rer. 63, 431-2 (1943).

\section{Appendix A. Notation and Terminology}

The spectroscopic notation used in this report is that adopted in Herzber'g's book [99] as modified by recommendations of the Triple Commission on Spectroscopy (J. Opt. Soc. Am. 43, 425-30 (1953); 52, 476-7 (1962); 53, 883-5 (1963)). A number of specific conventions used are itemized below.
(1) Wavenumber in $\mathrm{cm}^{-1}$ is denoted by $\sigma ; v$ is reserved for frequency in $s^{-1}$.

(2) $\mathrm{N}$ is total angular momentum of electrons and nuclei exclusive of spin (case $\left.b, b^{\prime}, d\right)$, formerly denoted by K.

(3) Rotational angular momentum of the nuclei, 
formerly denoted by $\mathrm{N}$, is now denoted by $\mathrm{O}$.

(4) Dissociation energy is written as $D^{0}$ or $\mathrm{D}^{e}$; rotational constants (for the zero level or equilibrium value, respectively) are denoted as usual by $\mathrm{D}_{0}$ and $\mathrm{D}_{e}$.

(5) A transition is always represented with a dash, as ${ }^{2} \Pi-{ }^{2} \Sigma$ transition. The upper state is always written first. $\leftarrow$ means absorption; $\rightarrow$ means emission, for an electronic, vibration-rotation, or rotational (here, microwave) transition.

(6) A perturbation by one state of another is indicated as e.g., $\left({ }^{1} \Delta^{1} \Pi\right)$ perturbation, following an early notation of Kovács. (Conventions used in some early papers include ${ }^{1} \Delta \times{ }^{1} \Pi$ or ${ }^{1} \Delta,{ }^{1} \Pi$.)

(7) A progression of bands is indicated as follows; e.g.,

(a) $v^{\prime \prime}=0$ progression

(b) $\left(v^{\prime}-0\right)$ progression.

(8) Reciprocal dispersion is given in $\AA / \mathrm{mm}$. However, following the colloquial use of many spectroscopists this quantity is referred to as dispersion.

(9) In the tables, wavelengths above $2000 \AA$ are air wavelengths unless otherwise specified; below $2000 \AA$ vacuum wavelengths are listed.

(10) The known band degradation is indicated by $\mathrm{R}$ (red-degraded) or $\mathrm{V}$ (violet-degraded) in the headings of section 3 as well as in tables 1 and 3 to 35 .

(11) Rotational constants in tables 36 to 52 are given in units of $\mathrm{cm}^{-1}$.

(12) Abbreviations used are listed on p. 2 In addition, zero-point energy is abbreviated by ZPE.

The following items apply to table 1 .

(1) Vibrational constants and term values $\mathrm{T}$ are assumed to be derived from data on band origins. (Herzberg [99] denotes these by the letter Z.) In conformity with Herzberg, $\mathrm{H}$ denotes constants derived from head measurements.

(2) $\left[r_{e}\right]$ means $r_{0} ;\left[\mathrm{B}_{e}\right]$ means $\mathrm{B}_{0} ;\left[\omega_{e}\right]$ means $\Delta \mathrm{G}\left(\frac{1}{2}\right)$, as in Herzberg's book.

(3) ( ) means uncertain.

(4) $\mathrm{T}_{0}$ is the mean height (in case of multiplets) above $\mathrm{X}, v=0, \mathrm{~J}=0$.

(5) States which are predissociated have $\operatorname{Pr}$ written in the column for dissociation products. (For $\mathrm{CO}$ all known predissociations are due to ${ }^{3} \mathrm{P}+{ }^{3} \mathrm{P}$ states.)

(6) All numerical data are in units of $\mathrm{cm}^{-1}$ unless otherwise indicated.

(7) References cited include only those from which the numerical data have been extracted. Other pertinent references are cited in the appropriate sections of this report.

(8) The tabulated molecular constants have been taken from the references cited in table 1 but are not inecessarily those which best fit the individual vibrational term values or rotational constants where these have been compiled from various sources.

(9) To avoid confusion of sign conventions several formulas are listed below:

Vibrational terms:

$$
\begin{aligned}
\mathrm{G}(v)=\omega_{e}(v+ & \left.\frac{1}{2}\right)-\omega_{e} x_{e}\left(v+\frac{1}{2}\right)^{2} \\
& +\omega_{e} y_{e}\left(v+\frac{1}{2}\right)^{3}+\omega_{e} z_{e}\left(v+\frac{1}{2}\right)^{4}
\end{aligned}
$$

i.e., a negative value of $\omega_{e} x_{e}$ from the table would mean a positive anharmonic term.

Rotational terms:

$$
\begin{array}{r}
\mathrm{F}_{v}(\mathrm{~J})=\mathrm{B}_{v} \mathrm{~J}(\mathrm{~J}+1)-\mathrm{D}_{\imath} \mathrm{J}^{2}(\mathrm{~J}+1)^{2} \\
+\mathrm{H}_{v} \mathrm{~J}^{3}(\mathrm{~J}+1)^{3}
\end{array}
$$

where $\left(-\mathrm{D}_{\nu}\right)$ is always $<0$, and

$$
\begin{aligned}
& \mathrm{B}_{v}=\mathrm{B}_{e}-\alpha_{e}\left(v+\frac{1}{2}\right)+\gamma_{e}\left(v+\frac{1}{2}\right)^{2} \\
& \quad+\delta_{e}\left(v+\frac{1}{2}\right)^{3} \\
& \mathrm{D}_{v}=\mathrm{D}_{e}+\beta_{e}\left(v+\frac{1}{2}\right)\left[\text { for } \mathrm{CO}, \mathrm{X}^{1} \Sigma^{+}, \beta<0\right] \\
& \mathrm{H}_{v} \sim \mathrm{H}_{e} .
\end{aligned}
$$

(10) Footnotes which give supplementary information pertaining to the individual electronic states are indicated at the end of table 1 and are identified by the electronic state. Table 1 has been left free of superscripts.

(11) The tabulated ZPE do not include the Dunham correction which would add an amount given by

$$
\mathrm{Y}_{00}=\frac{\mathrm{B}_{e}}{4}+\frac{\alpha_{e} \omega_{e}}{12 \mathrm{~B}_{e}}+\frac{\alpha_{e}^{2} \omega_{e}^{2}}{144 \mathrm{~B}_{e}^{3}}-\frac{\omega_{e} x_{e}}{4} .
$$

(For the ground state this correction is $0.600 \mathrm{~cm}^{-1}$.) 


\section{Appendix B. Physical Constants ${ }^{10-12}$ and Conversion Factors}

$$
\begin{aligned}
& c=2.997925 \times 10^{-10} \mathrm{~cm}^{-\mathrm{s}^{-1}} \\
& h=6.6256 \times 10^{-27} \mathrm{erg}^{-\mathrm{s}} \\
& \mathrm{N}_{0}=6.02252 \times 10^{23} \mathrm{~mole}^{-1} \\
& 1 \mathrm{eV}=8065.73 \mathrm{~cm}^{-1}=23.0609 \mathrm{k}-\mathrm{cal}-\mathrm{mole}^{-1} \\
& \mu_{\mathrm{A}}\left(\mathrm{C}^{12} \mathrm{O}^{16}\right)=6.85621 \\
& \mu_{\mathrm{A}}\left(\mathrm{CO}^{+}\right)=6.85603 \\
& \mu=\mu_{\mathrm{A}} / \mathrm{N}_{0}: \mu(\mathrm{CO})=1.13843 \times 10^{-23} \mathrm{~g} \\
& \mu\left(\mathrm{CO}^{+}\right)=1.13840 \times 10^{-23} \mathrm{~g}
\end{aligned}
$$

10 The molecular reduced masses are calculated from the data of Everling et al., Relative Nuclidic Masses, Nuclear Phys. 18, 529-69 (1960) which are based on the unified atomic weight scale with $\mathrm{C}^{12}=12$.

11 The mass $\mu_{\mathrm{A}}\left(\mathrm{CO}^{+}\right)$was calculated by assuming $5.49 \times 10^{-4}$ a.m.u. for the mass of the electron and assuming that ionization removes an electron from the $\mathrm{C}$ atom $\left(1 \mathrm{a}, \mathrm{m} . \mathrm{u} .=1 / 12\right.$ mass of $\left.\mathrm{C}^{12}\right)$.

12 The universal constants and conversion factors are those recommended by the NAS-NRC (Phys. Today, pp. 48-9 (Feb. 1964)).
Atomic weights:

$$
\begin{aligned}
\mathrm{C} & =12 \\
\mathrm{O} & =15.994915 \\
k_{e}(\mathrm{CO}) & =40.3930 \times 10^{-2} \omega_{e}^{2} \\
k_{e}\left(\mathrm{CO}^{+}\right) & =40.3920 \times 10^{-2} \omega_{e}^{2} \\
r_{e}(\mathrm{CO}) & =1.568031 \sqrt{\frac{1}{\mathrm{~B}_{e}}} \\
r_{e}\left(\mathrm{CO}^{+}\right) & =1.568052 \sqrt{\frac{1}{\mathrm{~B}_{e}}}
\end{aligned}
$$

$\left(\omega_{e}\right.$ in $\mathrm{cm}^{-1} ; k_{e}$ in dyne-cm ${ }^{-1}$ )

$\left(\mathrm{B}_{e}\right.$ in $\mathrm{cm}^{-1} ; r_{e}$ in $\left.\AA\right)$
The "Tabelle der Schwingungszahlen" of Kayser has been superseded by NBS Monograph 3, "Table of Wavenumbers," by Coleman, Bozman, and Meggers (1960) which is based on the 1953 formula for dispersion in standard air of Edlen. For low resolution work the older tables are adequate. 



\section{Announcement of New Publications in National Standard Reference Data Series}

Superintendent of Documents, Government Printing Office, Washington, D.C. 20402

Dear Sir :

Please add my name to the announcement list of new publications to be issued in the series: National Standard Reference Data Series-National Bureau of Standards.

Name

Company

Address

City State 


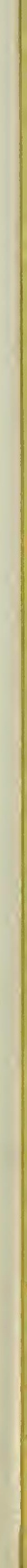




\section{The National Standard Reference Data Series}

National Standard Reference Data System-Plan of Operation, NSRDS-NBS 1. 15 cents Thermal Properties of Aqueous Uni-univalent Electrolytes, NSRDS-NBS 2. 45 cents Selected Tables of Atomic Spectra-Atomic Energy Levels and Multiplet Tables Si II, Si III, Si IV, NSRDS-NBS 3, Section 1.35 cents

Atomic Transition Probabilities-Hydrogen Through Neon, NSRDS-NBS 4, Volume I. $\$ 2.50$

The Band Spectrum of Carbon Monoxide, NSRDS-NBS 5. 70 cents

The above publications are available by purchase from the Superintendent of Documents, Government Printing Office, Washington, D.C., 20402. Orders from foreign countries should include an additional one-fourth of the purchase price, to cover postage for mailing.

See mailing list announcement on the last page of this publication. 
U.S. DEPARTMENT OF COMMERCE

POSTAGE AND FEES PAID

WASHINGTON, D.C. 20230

U.S. DEPARTMENT OF COMMERCE

OFFICIAL BUSINESS 